\title{
New hypotheses for the health-protective mechanisms of whole-grain cereals: what is beyond fibre?
}

\author{
Anthony Fardet ${ }^{1,2}$ \\ ${ }^{1}$ INRA, UMR 1019 Nutrition Humaine, F-63122 Saint-Genès-Champanelle, France \\ ${ }^{2}$ Clermont Université, UFR Médecine, UMR 1019 Nutrition Humaine, F-63000 Clermont-Ferrand, France
}

\begin{abstract}
Epidemiological studies have clearly shown that whole-grain cereals can protect against obesity, diabetes, CVD and cancers. The specific effects of food structure (increased satiety, reduced transit time and glycaemic response), fibre (improved faecal bulking and satiety, viscosity and SCFA production, and/or reduced glycaemic response) and $\mathrm{Mg}$ (better glycaemic homeostasis through increased insulin secretion), together with the antioxidant and anti-carcinogenic properties of numerous bioactive compounds, especially those in the bran and germ (minerals, trace elements, vitamins, carotenoids, polyphenols and alkylresorcinols), are today well-recognised mechanisms in this protection. Recent findings, the exhaustive listing of bioactive compounds found in whole-grain wheat, their content in whole-grain, bran and germ fractions and their estimated bioavailability, have led to new hypotheses. The involvement of polyphenols in cell signalling and gene regulation, and of sulfur compounds, lignin and phytic acid should be considered in antioxidant protection. Whole-grain wheat is also a rich source of methyl donors and lipotropes (methionine, betaine, choline, inositol and folates) that may be involved in cardiovascular and/or hepatic protection, lipid metabolism and DNA methylation. Potential protective effects of bound phenolic acids within the colon, of the B-complex vitamins on the nervous system and mental health, of oligosaccharides as prebiotics, of compounds associated with skeleton health, and of other compounds such as $\alpha$-linolenic acid, policosanol, melatonin, phytosterols and para-aminobenzoic acid also deserve to be studied in more depth. Finally, benefits of nutrigenomics to study complex physiological effects of the 'whole-grain package', and the most promising ways for improving the nutritional quality of cereal products are discussed.
\end{abstract}

Whole-grain wheat: Bioactive compounds: Physiological mechanisms: Health

\section{Introduction}

There is growing evidence that whole-grain cereal products protect against the development of chronic diseases. The most important of these in terms of public health are obesity $^{(1,2)}$, the metabolic syndrome ${ }^{(3,4)}$, type 2 diabetes ${ }^{(5,6)}$, $\mathrm{CVD}^{(7)}$ and cancers ${ }^{(8-12)}$. Whole-grain cereal consumption has also been shown to be protective against mortality, as was shown with inflammation-related death (i.e. noncardiovascular and non-cancer inflammatory diseases such as, for example, respiratory system diseases) ${ }^{(13)}$ and with cancer and $\mathrm{CVD}^{(4,14,15)}$. These conclusions are supported by the effects of consuming refined cereal products (bread, pasta and rice), as these have been associated with an increased risk of digestive tract, pharynx, larynx and thyroid cancers in northern Italians ${ }^{(16)}$. However, an association between a lower risk of developing a chronic disease and a high whole-grain cereal consumption does not mean a direct causal relationship and provides no information about the physiological mechanisms involved.

These metabolic diseases are related to our daily lifestyle, notably an unbalanced energy-rich diet lacking fibre and protective bioactive compounds such as micronutrients and phytochemicals. Today, it is agreed to advance that this is the synergistic action of the compounds, mainly contained in the bran and germ fractions of cereals, which is protective ${ }^{(17,18)}$. Some specific mechanisms are today well recognised. For example, food structure influences satiety and the slow release of sugars recommended for type 2 diabetes. Dietary fibre improves gut health, and the antioxidant and antiinflammatory properties of most phytochemicals can help

\footnotetext{
Abbreviations: AACC, American Association of Cereal Chemists; DW, dry weight; FRAP, ferric-reducing ability of plasma; GI, glycaemic index; GSH, reduced glutathione; GSSG, oxidised glutathione; RS, resistant starch; USDA, US Department of Agriculture.

Corresponding author: Dr Anthony Fardet, fax +33 473624638, email anthony.fardet@clermont.inra.fr
} 


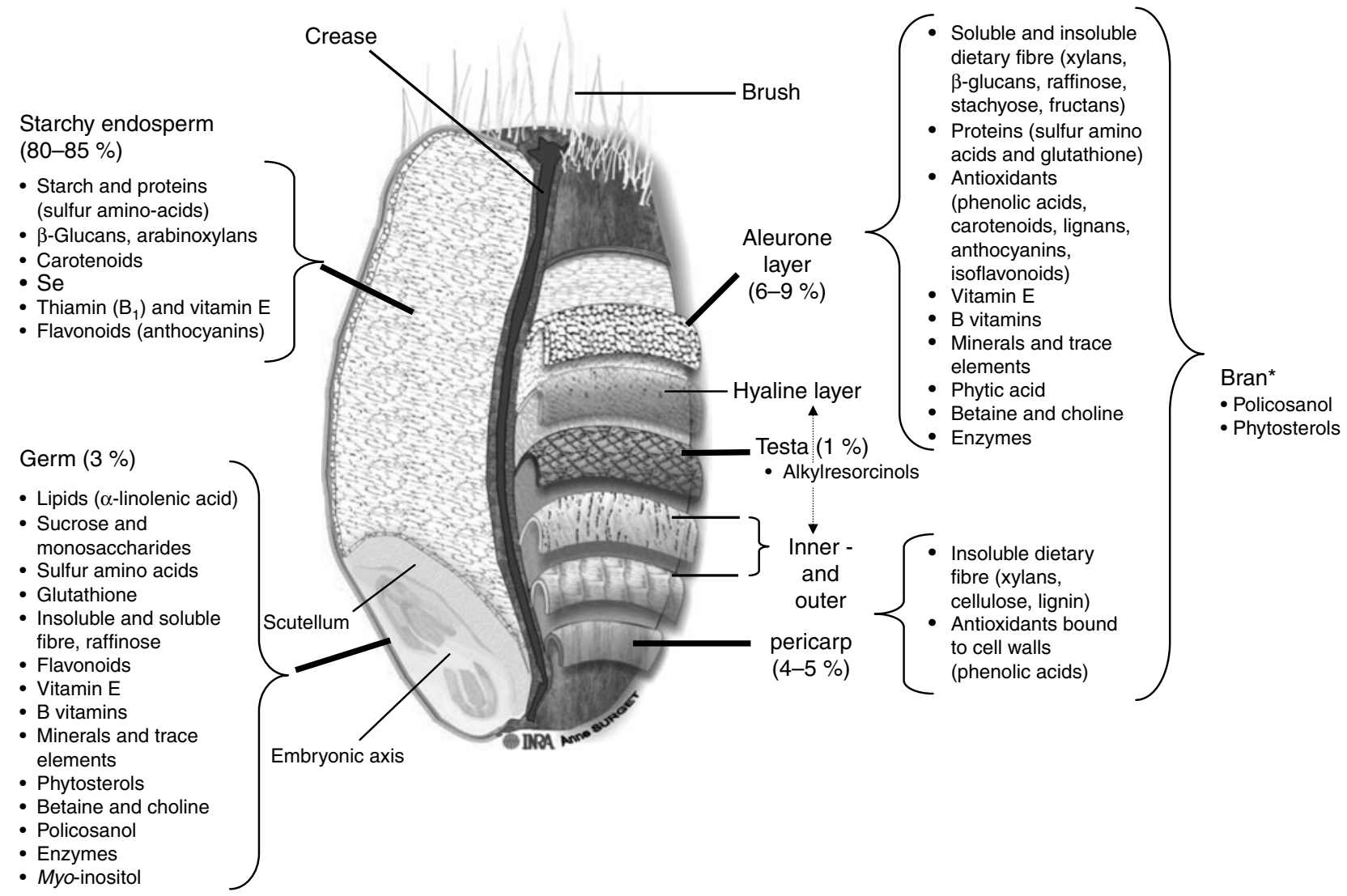

Fig. 1. The three wheat fraction (bran, germ and endosperm) with their main bioactive compounds as obtained from Tables 1 and 2. Whole-grain wheat has an heterogeneous struture with bioactive compounds unevenly distributed within its different parts (with permission from Surget \& Barron for original image ${ }^{(476)}$, and adapted from the brochure 'Progress in HEALTHGRAIN 2008', HealthGrain Project, European Community's Sixth Framework Programme, FOOD-CT-2005-514008, 2005-2010). * No published data on the precise locations of policosanol and phytosterols in a specific layer of the wheat bran fraction.

prevent cancer and CVD. However, the precise physiological mechanisms involved are far from being elucidated.

The main whole-grain cereals consumed worldwide are wheat, rice and maize, followed by oats, rye, barley, triticale, millet and sorghum. Whole-grain wheat, which is the focus of the present review, is composed of $10-14 \%$ bran, $2 \cdot 5-3.0 \%$ germ and $80-85 \%$ endosperm, depending on the intensity of the milling process. The bioactive compounds are unevenly distributed within these parts (Fig. 1), and this distribution also varies according to the type of cereal considered. Whole-grain cereals are a rich source of fibre and bioactive compounds. For example, whole-grain wheat contains about $13 \%$ dietary fibre and at least $2 \%$ bioactive compounds other than fibre (Table 1), which accounts for at least $15 \%$ of the whole grain. In the bran and germ fractions, still higher proportions are reached: about 45 and $18 \%$ of dietary fibre, and about $7 \%$ and at least $6 \%$ of bioactive compounds, respectively; which represents about $52 \%$ and at least $24 \%$ of these fractions. These proportions obviously depend on the cereal type. It is therefore easy to understand that refined cereal products that lack the bran and germ fractions have lost most of their protective compounds. For example, refining wholegrain wheat may lead to the loss of about $58 \%$ of fibre, $83 \%$ of $\mathrm{Mg}, 79 \%$ of $\mathrm{Zn}, 92 \%$ of Se, $70 \%$ of nicotinic acid, $61 \%$ of folates and $79 \%$ of vitamin $\mathrm{E}^{(19)}$.
However, the exact nature of the positive physiological effects exerted by whole-grain cereal products remains unresolved because of the huge number of phytochemicals and biological effects involved (Tables 2 and 3). The most significant of them in wheat, besides fibre, are n-3 fatty acids, sulfur amino acids, oligosaccharides (stachyose, raffinose and fructans), lignin, minerals, trace elements, vitamins $\mathrm{B}$ and $\mathrm{E}$, carotenoids, polyphenols (especially phenolic acids such as ferulic acid and smaller amounts of flavonoids and lignans), alkylresorcinols, phytic acid, betaine, total choline-containing compounds, inositols, phytosterols, policosanol and melatonin. Each one of these compounds has numerous physiological functions and recognised health benefits (Tables 3 and 4). While studying each compound separately, the main approach used to date, may well be unavoidable, it also involves considerable risk. This is because it ignores two important factors. One is the importance of synergy between the actions of compounds which is poorly characterised and more difficult to assess than the biological action of an isolated compound. The second is the importance of the cereal matrix and its influence on the accessibility of compounds in the digestive tract and hence on their availability within the organism. Indeed, little is often known of the bioavailability of many bioactive compounds derived from complex cereal products (Table 2). Thus, the amount of a particular compound in 
Table 1. Average content of the major bioactive compounds in whole-grain wheat and wheat bran and germ fractions (\%)*

\begin{tabular}{|c|c|c|c|}
\hline Bioactive compound & Whole-grain wheat $†$ & Wheat bran $†$ & Wheat germ $\dagger$ \\
\hline$\alpha$-Linolenic acid (18:3n-3) & $-\ddagger$ & $0 \cdot 16$ & 0.53 \\
\hline Sulfur compounds & 0.5 & 0.7 & $1 \cdot 2$ \\
\hline Total free glutathione§ & 0.007 & 0.038 & 0.270 \\
\hline Dietary fibrell & $13 \cdot 2$ & $44 \cdot 6$ & $17 \cdot 7$ \\
\hline Lignins & 1.9 & $5 \cdot 6$ & 1.5 \\
\hline Oligosaccharides $\rrbracket$ & 1.9 & $3 \cdot 7$ & $10 \cdot 1$ \\
\hline Phytic acid & 0.9 & $4 \cdot 2$ & $1 \cdot 8$ \\
\hline Minerals and trace elements & $1 \cdot 12$ & $3 \cdot 39$ & 2.51 \\
\hline Vitamins & 0.0138 & 0.0398 & 0.0394 \\
\hline B vitamins & 0.0091 & 0.0303 & 0.0123 \\
\hline Vitamin E (tocopherols and tocotrienols) & 0.0047 & 0.0095 & 0.0271 \\
\hline Carotenoids & 0.00034 & 0.00072 & $-\ddagger$ \\
\hline Polyphenols & $0 \cdot 15$ & $1 \cdot 10$ & $>0.37$ \\
\hline Phenolic acids & $0 \cdot 11$ & 1.07 & $>0.07$ \\
\hline Flavonoids & 0.037 & 0.028 & 0.300 \\
\hline Lignans & 0.0004 & 0.0050 & 0.0005 \\
\hline Alkylresorcinol & 0.07 & 0.27 & $-\ddagger$ \\
\hline Betaine & 0.16 & $0 \cdot 87$ & $0 \cdot 85$ \\
\hline Total choline & $0 \cdot 12$ & $0 \cdot 17$ & 0.24 \\
\hline Total free inositols (myo- and total chiro-inositols) & 0.022 & 0.025 & $>0.011$ \\
\hline Phytosterols & 0.08 & $0 \cdot 16$ & 0.43 \\
\hline Policosanol + melatonin + para-aminobenzoic acid & 0.00341 & 0.00290 & $>0.00186$ \\
\hline Total & $>15.4$ & 51.5 & $>23.9$ \\
\hline Subtotal (without dietary fibre) & $>2 \cdot 2$ & $6 \cdot 9$ & $>6 \cdot 2$ \\
\hline
\end{tabular}

whole-grain cereals is rarely the same as the amount that is available to exert a given physiological action, in contrast to the result of consuming the free compound.

There may be many protective physiological mechanisms associated with consuming whole-grain cereal because of the high number of protective compounds. They may be mechanical within the digestive tract (insoluble fibre can increase transit time and faecal bulking), hormonal ( $\mathrm{Zn}, \mathrm{Se}$ and nicotinic acid participating in hormone activation and synthesis), antioxidative (almost all micronutrients), antiinflammatory (for example, $n-3 \alpha$-linolenic acid, $\mathrm{Cu}$ and ferulic acid), anti-carcinogenic (almost all micronutrients), or linked to gene regulation (for example, flavonoids), cell signalling (for example, polyphenols and redox status), energy metabolism (for example, the B-complex vitamins) and effects on enzymes (for example, some minerals and trace elements) (Table 3).

The main objective of the present paper is to propose new hypotheses for exploring the mechanisms behind the protective actions of whole-grain cereals using wheat as the main example. I have therefore exhaustively itemised all the bioactive compounds in whole-grain wheat and in the two fractions that are usually removed during refining: bran and germ. I have also listed their contents (range) in wheat, their bioavailability when obtained from complex wholegrain wheat products, their potential physiological effect(s) and the resulting health outcomes, with particular attention to some compounds that are specific to cereals other than wheat. The proposed new hypotheses are based on the action of compounds that are all bioactive when tested alone in their free form, such as the B vitamins, lignin, phytic acid, betaine, choline-containing compounds, inositols, policosanol, melatonin, para-aminobenzoic acid, sulfur amino acids, $\alpha$-linolenic acid, phytosterols and some oligosaccharides.

First, I define the term 'whole-grain cereal products' and then examine the presently accepted mechanisms for explaining the role played by whole-grain cereals in preventing chronic diseases, as identified by studies on human subjects (for example, the importance of food structure and antioxidants), on rats (for example, the anticarcinogenic property of many phytochemicals) and in vitro (cell-associated mechanisms). I then discuss my new hypotheses that are based on recent findings and on the potential physiological effects of whole-grain cereal compounds. I develop a broader view of the well-known antioxidant hypothesis that takes into account the actions of polyphenols on cell signalling and gene regulation in relation to the redox status. I review recent publications that have also revealed the great potential of the nutrigenomic approach for extending our knowledge of the protective mechanisms associated with complex foods. Finally, I briefly review the ways by which the nutritional quality of cereal products can be improved so as to optimally preserve the protective properties of whole-grain cereals.

\section{What are whole-grain cereal products?}

\section{Definition}

The American Association of Cereal Chemists (AACC) gave the following scientific and botanical definition in 1999: 'Whole grains shall consist of the intact, ground, cracked or flaked caryopsis, whose principal anatomical 


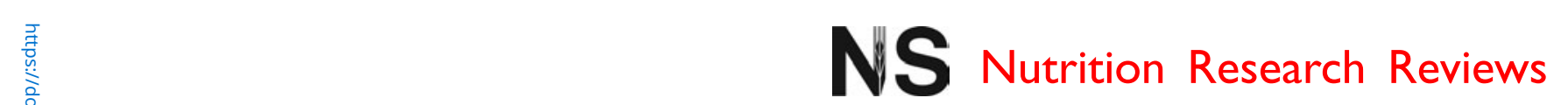

Table 2. Content, apparent absorption and fermentability of bioactive compounds and fibre from whole-grain wheat and wheat bran and germ fractions ${ }^{*}$

\begin{tabular}{|c|c|c|c|c|c|}
\hline Bioactive compound & $\begin{array}{l}\text { Content in whole } \\
\text { grain (per } 100 \mathrm{~g}) \dagger\end{array}$ & $\begin{array}{l}\text { Apparent absorption or degree } \\
\text { of fermentation in crude or processed } \\
\text { whole-grain wheat }(\%)\end{array}$ & $\begin{array}{l}\text { Content in bran } \\
\text { (per } 100 \mathrm{~g}) \dagger\end{array}$ & $\begin{array}{l}\text { Apparent absorption or degree } \\
\text { of fermentation in crude or } \\
\text { processed wheat bran (\%) }\end{array}$ & $\begin{array}{l}\text { Content } \\
\text { in germ } \\
\text { (per } 100 \mathrm{~g}) \dagger\end{array}$ \\
\hline \multicolumn{6}{|l|}{$n-3$ Fatty acids $(\mathrm{g} / 100 \mathrm{~g})$} \\
\hline$\alpha$-Linolenic acid $(18: 3 n-3)$ & $-\ddagger$ & $-\ddagger$ & $0 \cdot 16$ & $-\ddagger$ & $0.47-0.59$ \\
\hline \multicolumn{6}{|l|}{ Sulfur compounds } \\
\hline Reduced glutathione $(\mathrm{mg} / 100 \mathrm{~g}) \S$ & $1.04-5.74$ & Negligible in humans when freeł & $1 \cdot 7-19 \cdot 4$ & Negligible in humans when freeł & $19 \cdot 4-245 \cdot 7$ \\
\hline Oxidised glutathione $(\mathrm{mg} / 100 \mathrm{~g}) \S$ & $0.86-2.88$ & _ & $6 \cdot 1-21 \cdot 4$ & - & $15 \cdot 3-122 \cdot 4$ \\
\hline Methionine $(\mathrm{g} / 100 \mathrm{~g})$ & $0.17-0.24$ & - & $0.20-0.29$ & - & $0.39-0.58$ \\
\hline Cystine $(\mathrm{g} / 100 \mathrm{~g})$ & $0.19-0.40$ & - & $0.32-0.45$ & - & $0.35-0.61$ \\
\hline \multicolumn{6}{|l|}{ Sugars $(g / 100 \mathrm{~g})$} \\
\hline Monosaccharides & $0.26-1.30$ & - & $0.14-0.63$ & - & $0.6-1.5$ \\
\hline Sucrose & $0.60-1.39$ & - & $1 \cdot 8-3.4$ & - & $7 \cdot 7-16 \cdot 0$ \\
\hline \multicolumn{6}{|l|}{ Fibre $(g / 100 \mathrm{~g})$} \\
\hline Total & $9 \cdot 0-17 \cdot 3$ & 34 in humans $\|$ & $35 \cdot 7-53 \cdot 4$ & $\begin{array}{l}34-56 \text { in humans; } 37-49 \\
\text { in rats; } 42-65 \text { in pigs } \|\end{array}$ & $10 \cdot 6-24 \cdot 7$ \\
\hline Insoluble & $9.5-11.4$ & - & $32 \cdot 4-41 \cdot 6$ & 42 in rats $\|$ & $8 \cdot 5-18 \cdot 6$ \\
\hline Soluble & $1 \cdot 1-3 \cdot 2$ & - & $1 \cdot 3-5 \cdot 8$ & 73 in rats $\|$ & $2 \cdot 1-6 \cdot 1$ \\
\hline Cellulose & $2 \cdot 1-2 \cdot 8$ & 20 in humans $\|$ & $6.5-9.9$ & $\begin{array}{l}6-23 \text { in humans; } 14-24 \\
\text { in pigs and rats\| }\end{array}$ & $7 \cdot 5$ \\
\hline Hemicellulose & $8 \cdot 6$ & 46 in humans $\|$ & $20 \cdot 8-33 \cdot 0$ & $\begin{array}{l}50-54 \text { in humans; } 47-74 \\
\text { in pigs and rats } \|\end{array}$ & $6 \cdot 8$ \\
\hline Lignins & $0.9-2.8$ & 4 in humans $\|$ & $2 \cdot 2-9 \cdot 0$ & 0 in humans $\ddagger ; 0-4$ in rats $\|$ & $1.3-1.6$ \\
\hline Fructans & $0 \cdot 6-2 \cdot 3$ & - & $0 \cdot 6-4 \cdot 0$ & - & $1 \cdot 7-2 \cdot 5$ \\
\hline Raffinose & $0.13-0.59$ & 97-99 in dogs fed a soyabean mealł & $1.08-1.32$ & $\begin{array}{l}\text { Almost completely fermented } \\
\text { when freeł }\end{array}$ & $5 \cdot 0-10 \cdot 9$ \\
\hline Stachyose & $0.05-0.17$ & 97-99 in dogs fed a soyabean mealł & $0.04-0.36$ & $\begin{array}{l}\text { Almost completely fermented } \\
\text { when freeł }\end{array}$ & - \\
\hline Arabinoxylans & $1 \cdot 2-6 \cdot 8$ & - & $5 \cdot 0-26 \cdot 9$ & $\begin{array}{l}49 \text { arabinose in humans; } \\
71 \text { xylose in humans }\end{array}$ & $5 \cdot 6-9 \cdot 1$ \\
\hline Water-extractable & $0 \cdot 2-1 \cdot 2$ & - & $0 \cdot 1-1 \cdot 4$ & - & 0.4 \\
\hline$\beta$-Glucans & $0.2-4.7$ & - & $1 \cdot 1-2 \cdot 6$ & - & - \\
\hline $\begin{array}{l}\text { Phytic acid } \\
\text { (hexakisphosphate; g/100 g) }\end{array}$ & $0.3-1.5$ & $\begin{array}{l}\text { Poorly absorbed in humans; } 54-79 \\
\text { degraded in human } \\
\text { subjects fed whole bread; } 79 \% \\
\text { absorbed as free compound in rats } ¥ \mathbb{9}\end{array}$ & $2 \cdot 3-6 \cdot 0$ & $\begin{array}{l}58-60 \text { degraded into lower } \\
\text { inositol phosphates in } \\
\text { ileostomates fed raw wheat bran }\end{array}$ & $1 \cdot 4-2 \cdot 2$ \\
\hline \multicolumn{6}{|l|}{ Minerals (mg/100g) } \\
\hline $\mathrm{Fe}$ & $1 \cdot 0-14 \cdot 2$ & $1-20$ in human or usual diets $\ddagger$ & $2 \cdot 5-19 \cdot 0$ & $\begin{array}{l}3.8 \text { in human subjects fed } \\
\text { wheat bran rolls }\end{array}$ & $3 \cdot 9-10 \cdot 3$ \\
\hline $\mathrm{Mg}$ & $17-191$ & $\begin{array}{l}21-28 \text { in human subjects fed brown } \\
\text { bread diet; } 70 \text { in rats }\end{array}$ & $390-640$ & - & $200-290$ \\
\hline $\mathrm{Zn}$ & $0.8-8.9$ & $17-20$ in humans; $19-95$ in rats & $2 \cdot 5-14 \cdot 1$ & - & $10-18$ \\
\hline $\mathrm{Mn}$ & $0.9-7.8$ & Very low $\ddagger$ & $4-14$ & Very low $\ddagger$ & $9-18$ \\
\hline $\mathrm{Cu}$ & $0.09-1.21$ & $\begin{array}{l}62-85 \text { in humans; } 16 \\
\text { as free compound in rats }\end{array}$ & $0 \cdot 84-2 \cdot 20$ & - & $0 \cdot 70-1.42$ \\
\hline $\mathrm{Se}$ & $0.0003-3$ & $81-85$ in rats & $0.002-0.078$ & $60-80$ in rats/free sodiumselenite & $0.001-0.079$ \\
\hline $\mathrm{P}$ & $218-792$ & $41-55$ in humans fed brown bread diet & $900-1500$ & $\begin{array}{l}41-56 \text { in human subjects fed } \\
\text { sodium phytateł }\end{array}$ & $770-1337$ \\
\hline $\mathrm{Ca}$ & $7-70$ & $82 \%$ in humans; $43-93$ in rats & $24-150$ & $22 \%$ in humans & $36-84$ \\
\hline $\mathrm{Na}$ & $2-16$ & - & $2-41$ & - & $2-37$ \\
\hline $\mathrm{K}$ & $209-635$ & - & $1182-1900$ & - & $788-1300$ \\
\hline \multicolumn{6}{|l|}{ Vitamins (mg/100 g) } \\
\hline Thiamin $\left(\mathrm{B}_{1}\right)$ & $0.13-0.99$ & 91 in rats/free thiamin mononitrate & $0.51-0.80$ & - & $0 \cdot 8-2 \cdot 7$ \\
\hline
\end{tabular}


Riboflavin $\left(\mathrm{B}_{2}\right)$

Nicotinic acid $\left(B_{3}\right)$

Pantothenic acid $\left(B_{5}\right)$

Pyridoxine $\left(\mathrm{B}_{6}\right)$

Biotin $\left(\mathrm{B}_{8}\right)$

Folate $\left(\mathrm{B}_{9}\right)$

Tocopherols + tocotrienols $(E)$

Total tocopherols

$\alpha$-Tocopherol

Total tocotrienols

Phylloquinone $(\mathrm{K})$

Carotenoids (mg/100 g)

Total

$\beta$-Carotene

Lutein

Zeaxanthin

$\beta$-Cryptoxanthin

Polyphenols

Phenolic acids $(\mathrm{mg} / 100 \mathrm{~g})^{\star \star}$

Total

Extractable (free and conjugated)

Bound

Ferulic acid $(\mathrm{mg} / 100 \mathrm{~g})$

Total

Extractable (free and conjugated)

Bound

Dehydrodiferulic acid

Dehydrotrimer ferulic acid

Flavonoids $(\mathrm{mg} / 100 \mathrm{~g})$

Free $(\mathrm{mg} / 100 \mathrm{~g})$

Bound $(\mathrm{mg} / 100 \mathrm{~g})$

Anthocyanins $(\mathrm{mg} / 100 \mathrm{~g})$

soflavonoids $(\mu \mathrm{g} / 100 \mathrm{~g})$

Lignans (mg/100 g)

Others

Alkylresorcinols (mg/100 g)

Betaine (mg/100 g)

Total choline ๆ $(\mathrm{mg} / 100 \mathrm{~g})$

Phytosterols (mg/100 g)

Inositols

Total chiro-inositol††† (mg/100 g)

Free myo-inositol $(\mathrm{mg} / 100 \mathrm{~g})$ 拉
$0.7-4.9$

$0.04-0.31$

$1 \cdot 9-11 \cdot 1$

$0.7-2 \cdot 0$

0.09-0.66

$0.002-0.011$

$0.014-0.087$

$2 \cdot 3-7 \cdot 1$

$1 \cdot 06-2 \cdot 89$

$0.34-3.49$

$1.09-4.49$

$0.044-0.626$

$0.005-0.025$

$0.026-0.383$

$0.009-0.039$

$0.001-0.013$

$16-102$

$5-39$

$14-78$

14-64

$1 \cdot 5-76 \cdot 0$

$2 \cdot 6-3.5$

$30-43 \ddagger \ddagger$

$2 \cdot 2-4 \cdot 9 \ddagger$

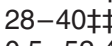

$0.5-52.4$

$14 \cdot 8\|\|$

$0 \cdot 2-0 \cdot 6$

$12-129$

22-291

$27-195$

$57-98$

17

$1.9-7.5$
95 as oral supplement in human subjectsł

Low, since mostly bound‡

$0.21-0.80$

$13 \cdot 6-35 \cdot 9$

About 50 in human/average American diet

71-79 in human/average American die

as compared with free compound $\neq$

very low $\ddagger$

70 in humans as free compound

-

$-$

See free/soluble-conjugated and bound

Probably high

See wheat bran

Low: $3.4 \%$ urinary excretion in rats

High in rat small intestine

Low: action of small intestine esterases

See wheat bran $\ddagger$

-
-
-
-
-
-
-
$60-79$ in pig small intestine fed
$\quad$ whole-grain rye bread
-
$\overline{-}$
Weakly absorbed from the gut

Apparently high in humans/free compound

Apparently high in rat as free compound
$2 \cdot 2-4 \cdot 1$

$0 \cdot 70-1 \cdot 30$

0.0440

$0.088-0.373$

9.5

$0 \cdot 13-2 \cdot 84$

$0 \cdot 13-10$
$7 \cdot 10$

$0.002-0.083$

$0.25-1 \cdot 18$

$0.003-0.010$

$0.050-0.180$

$0.025-0.219$

$0.018-0.064$

$761-1384$

46-63††

$148-340+\uparrow$

$138-631$

$1 \cdot 3-23 \cdot 1$

$122-286$

$13-230$

$15-25$

$15-4$

$0.9-48.0$

$3 \cdot 8-10 \cdot 4$

$2 \cdot 8-6 \cdot 7$

215-323

$230-1506$

$74-270$

$121-195$

nd

$14 \cdot 0-36 \cdot 4$
$27-38$ in humans (nicotinic acid

$0.50-0.80$ concentrate)

Unavailable in humans

2.7

0.49-1.98

Very low $\ddagger$

Low

Not readily available

0.0172

$0.14-0.70$

$3.1-22$

$1 \cdot 3-1 \cdot 6$

$0.003-0.350$

$\overline{0} .06$

$-$

See free/soluble-conjugated

and bound

Probably high

33 in pig; partially/slowly

solubilised within human

model colon

Low: $2 \cdot 0-5 \cdot 7 \%$ urinary excretion in humans and

$3.9 \%$ urinary excretion in rats

High: $27 \cdot 8-78.9 \%$ urinary

excretion in humans; high

in rat small intestine

Low: action of small intestine

esterases

Undetectable in human plasma;

free diferulic acid can be

absorbed from the gut in rats

-

$-$

$45-71 \%$ from ileostomy effluents in humans fed rye bran soft/crisp bread

Weakly absorbed from the gutł

$306-1395$

$152-330$

$410-450$

$8 \cdot 5-13 \cdot 3$ 


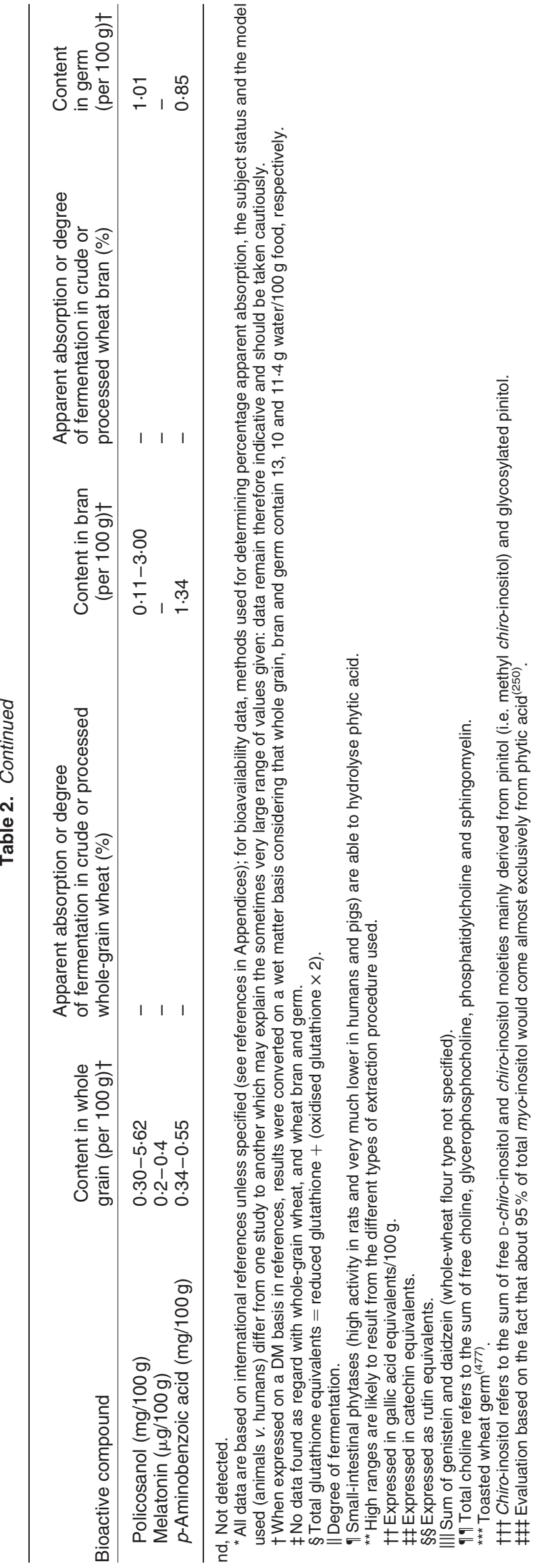

components - the starchy endosperm, germ and bran - are present in the same relative proportions as they exist in the intact caryopsis'(20). The definition given by the Whole Grains Council in May 2004 includes processed food products: 'Whole grains or foods made from them contain all the essential parts and naturally-occurring nutrients of the entire grain seed. If the grain has been processed (e.g. cracked, crushed, rolled, extruded, and/or cooked), the food product should deliver approximately the same rich balance of nutrients that are found in the original grain seed ${ }^{(21)}$. The US Food and Drug Administration published a Draft Guidance on Whole-grain Label Statements in 2006 that adopted the international AACC definition and included amaranth, barley, buckwheat, bulgur, maize (including popcorn), millet, quinoa, rice, rye, oats, sorghum, teff, triticale, wheat and wild rice; pearled barley was not included because some outer layers of the bran fraction are removed $^{(22)}$. Pseudocereals such as amaranth, buckwheat and quinoa have similar macronutrient compositions (carbohydrates, proteins and lipids), and are used in the same traditional ways as cereals ${ }^{(23,24)}$. The response to the US Food and Drug Administration Draft Guidance by the AACC International recommended that some traditional cereals such as 'lightly pearled barley, grano (lightly pearled wheat), nixtimalized corn and bulgur that has been minimally processed be also classified as whole grains, ${ }^{(23)}$, making allowance for small losses of components that occur through traditional processing. The Whole Grain Task Force stated in 2008 that it 'supports the use of the term whole-grain for products of milling operations that divide the grain into germ, bran and endosperm, but then recombine the parts into their original proportions before the flour leaves the mill ${ }^{(24)}$. However, as I will explain later, most of the products defined as whole-grain foods in studies showing the health benefits of whole-grain cereals are made of recombined whole-grain flours ${ }^{(24)}$, which rarely contain the same proportions of bran, germ and endosperm as the intact grain before milling. Thus, the germ fraction is almost always removed because its high lipid content (about $9 \%$ ) may go rancid upon storage ${ }^{(25)}$. Processing whole-grain cereals also leads to losses of bioactive compounds so they cannot really deliver 'approximately the same rich balance of nutrients that are found in the original grain seed ${ }^{(21)}$. Thus, if researchers had referred strictly to the definitions given above, few studies could have concluded that whole-grain cereal foods protect human health. Alternative definitions have therefore been proposed by the Whole Grain Task Force in which 'as they exist in the intact caryopsis' in the AACC definition is replaced by 'as found in the least-processed, traditional forms of the edible grain kernels' or completed by adding 'as they exist in the intact caryopsis to the extent feasible by the best modern milling technology' ${ }^{(24)}$. This last definition is probably the best adapted to our Western country technologies. But none of these alternative definitions has been adopted to date and there is still no official international definition of whole-grain cereal products in Europe.

\section{What proportions?}

Finally, the proportion of whole grains that must be present in a cereal product needs to be defined for it to be considered 
Table 3. Main physiological functions, potential protective mechanisms and health benefits of isolated bioactive compounds found in whole-grain wheat, rice and oat

Bioactive compounds (degree of

significance)

$n$-3 Fatty acids

$\alpha$-Linolenic acid $(18: 3 n-3)(++)$

Main physiological functions and potential protective mechanisms

Beneficial effects on blood clotting, thrombosis, blood pressure and inflammation: for example, anti-atherosclerotic effect via inhibition of oxidative stress-mediated $\mathrm{CD} 40 \mathrm{~L}$ (protein with inflammatory and prothrombotic property) up-regulation, suppresses levels of arachidonic acid $(20: 4 n-6)$ and eicosanoids in tissues (such as lung) and plasma phospholipids and the synthesis of pro-thrombotic cyclooxygenase-derived products (thromboxane A2 and B2, PGE2), reduces plasma TAG, inhibits synthesis of cytokines and mitogens; essential constituent of neuronal cells and retina; precursor in vivo of potentially protective DHA $(22: 6 n-3)$ and EPA $(20: 5 n-3)$; stimulates immune system via cell signalling and gene expression

Sulfur compounds

Reduced glutathione GSH $(+)$

Methionine $(++)$

Cystine (+)

Undigestible carbohydrate Insoluble fibre $(+++) \dagger$

(cellulose, hemicellulose)

Soluble fibre $(++) \dagger$

(for example, $\beta$-glucans,

arabinoxylans)

Resistant starch $(+++) \dagger$

Oligosaccharides $(++)$

(fructans, raffinose, stachyose)

Phytic acid $(+++)$ (also named myo-inositol hexakisphosphate)
Strong antioxidant; detoxification of toxic electrolytic metabolites of xenobiotics and of reactive oxygen intermediates generated intracellularly and at sites of inflammation; binding with cellular mutagens; important role in cellular immune function and as source of cysteine for various organs

Precursor of S-adenosyl methionine, the universal methyl donor, and of glutathione; intermediate in the biosynthesis of cysteine, carnitine, taurine, lecithin, phosphatidylcholine, and other phospholipids; methyl donor; may possess antioxidant activity; lipotrope

Reduced to two cysteine residues upon absorption; cell signalling through reactive cysteine residues in proteins; antioxidant; precursor of glutathione; constituent of the antioxidant metallothionein; many metal cofactors in enzymes are bound to the thiolate substituent of cysteinyl residues; precursor to $\mathrm{Fe}-\mathrm{S}$ clusters (role in the oxidation-reduction reactions of mitochondrial electron transport); increases protein stability in the harsh extracellular environment

Delivers antioxidant-bound phenolics to the colon; carcinogen binding and/or diluting; increases gut transit and faecal bulking; satiating effect

Decreases glycaemia through a delayed gastric emptying and glucose absorption rate; reduces cholesterolaemia through a possible effect of propionate (yielded by fibre fermentation) upon hepatocyte cholesterol and NEFA synthesis; improves insulin response; reduces bile acid reabsorption; produces SCFA

Produces high levels of butyrate, a tumour-growth suppressor; decreases glycaemia, cholesterolaemia and energy intake; promotes lipid oxidation and metabolism; prebiotic effect

Prebiotic (effect on bacterial metabolism: for example, bifidogenic); cholesterollowering through SCFA production, especially propionate; decrease glycaemia (through reduced hepatic gluconeogenesis by propionate) and triacylglycerolaemia; limit TAG accumulation in liver: effect on lipogenesis through exposure to propionate and reduced insulin/glucagon levels; produce butyrate, a tumour-growth suppresor; increase the absorption of minerals within colon; stimulate the immune system; control blood ammonia levels

Antioxidant: chelates various metals (for example, suppresses damaging Fe-catalysed redox reactions), inhibits xanthine oxidase, suppresses oxidant damage to the intestinal epithelium, and interferes with the formation of ADP-Fe-oxygen complexes that initiate lipid peroxidation; prevents the formation of carcinogens and blocks the interaction of carcinogens with cells; controls cell division and reduces cell proliferation rate; increases the immune response by enhancing the activity of natural killer cells; may be involved in cellular and nuclear signalling pathways; mportant source of $\mathrm{P}$; inhibitor for renal stone development; hypoglycaemic (for example, by chelating $\mathrm{Ca}$, an $\alpha$-amylase cofactor) and cholesterol-lowering (by
Potential health protection

CVD; retinal and brain development; inflammatory bowe disease (Crohn's); breast and colon cancers; mild hypertension; mental health (for example, depression and anxiety); rheumatoid arthritis

Some cancers (for example, oral); diseases associated with imbalance of glutathione (for example, HIV, ageing, hepatic cirrhosis, cystic fibrosis and lung and neurodegenerative disorders)

Neural tube defects; cognitive impairment; atherosclerosis; muscular wasting

Muscular wasting; normal hair and nail development

Gut health; colon cancer; obesity and weight regulation

Type 2 diabetes; CVD; gut health; colon cancer

Type 2 diabetes; CVD; colon health and cancer; body weight; gallstones

Gut health; colon cancer; CVD; lifespan; weight reduction; hepatic encephalopathy (nervous troubles) and steatosis

Various cancers (for example, colon and breast cancers) type 2 diabetes; CVD (for example, age-related aorta calcification); kidney health (renal stone development) hypercalciura; acute $\mathrm{Pb}$ poisoning; dental caries 


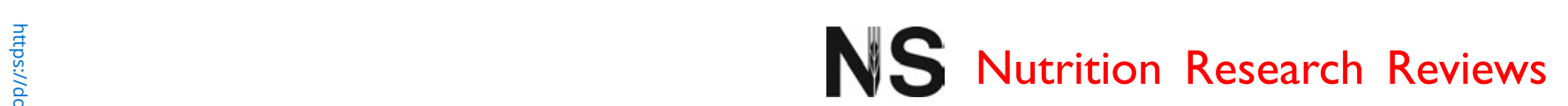

Table 3. Continued

Bioactive compounds (degree of significance)

Lignins $(+++)$

Minerals and trace elements $\mathrm{Fe}(++) \dagger$

$\operatorname{Mg}(+++) \dagger$

$\mathrm{Zn}(++) \dagger$

$\operatorname{Mn}(++)$
Main physiological functions and potential protective mechanisms

Potential health protection

binding $\mathrm{Zn}$ and decreasing $\mathrm{Zn}: \mathrm{Cu}$ ratio) effects; affects the metabolic and detoxification capacity of the liver; reduces blood glucose and lipid, and hepatic lipid levels; inhibits calcification of cardiovascular system (lower level of $\mathrm{Ca}$ in aorta); prevention of platelet aggregation; high affinity for hydroxyapatite; adsorption onto Ca-based crystals; in vitro effect on gene expression through chromatin remodelling

Antioxidant due to phenolic hydroxyl groups; adsorb dietary carcinogens and reduce carcinogen exposure; reduce bile acid reabsorption as a bile salt-sequestrating agent: possibly reduce fat absorption and the formation of carcinogenic metabolites from bile salts, and increase cholesterol turnover; source of enterolactone, a phytooestrogenic mammalian lignan

Cofactor with several enzymes involved in energy metabolism and thermoregulation: for example, catalase cofactor in the production of $\mathrm{O}_{2}+2 \mathrm{H}_{2} \mathrm{O}$ from $\mathrm{H}_{2} \mathrm{O}_{2}$, involvement in the Krebs cycle, oxygen transport as $\mathrm{Hb}$ and myoglobin constituent, and electron carrier within cytochromes; close association between the activities of Fe-containing oxidase pathways in muscle and endurance; role in collagen synthesis, and bone formation and resorption (for example, bone mineral density in synthesis, and bone formation and resorption (for example, bone mineral density in
post-menopause); role in cell-mediated immunity and phagocytosis; role in vitamin metabolism; improves developmental scores in Fe-deficient infants; might reduce increase in lipid peroxides and liver/serum TAG, cholesterol and phospholipids; deficiency associated with obesity

Second most abundant intracellular cation; constituent of several metalloenzymes with a role in cellular functions: glycolysis, DNA transcription, protein synthesis and oxidative phosphorylation; essential for all ATPase activity; necessary for coenzyme A reaction (for example, increases enzyme activity in the liver: lipotrope-like effect); A reaction (for example, increases enzyme activity in the liver: lipotrope-like effect),
role in neurotransmission, gut transit/cardiac rhythm/blood pressure regulation, role in neurotransmission, gut transit/cardiac rhythm/blood pressure regulation,
platelet aggregability and insulin sensitivity; improved glucose uptake, glucose metabolic clearance rate, and oxidative glucose metabolism; cholesterol-lowering and may reduce hypertriacylglycerolaemia; antioxidant (for example, against lipid peroxidation); favours $\mathrm{Ca}$ fixation in bones and muscle relaxing; activates alkaline phosphatase; psycho-relaxing; anti-inflammatory and anti-allergic effects; favours thermoregulation; important role in inducing some angiogenesis-related mechanisms; direct inhibition of calcium oxalate crystallisation in the urine

Superoxide dismutase and alkaline phosphatase cofactor; antioxidant; chemical inactivator: inhibits the formation of active carcinogenic compounds (for example, conversion of nitrosamines from nitrite in the stomach and $\mathrm{Zn}$-binding compounds); lymphocyte $\mathrm{T}$ and hormone activator; participates in numerous enzymic reactions (more than 200 enzymes) in relation to carbohydrate, lipid and protein metabolism; role in neurotransmission; DNA stabilisation; markedly modulates mechanisms of the pathology of inflammatory diseases; influences gene expression, cell development and replication; role in cell signalling within salivary gland, prostate, immune system, intestine and endothelial cells (for example, NF-кB and activator protein-1); key factor in reproductive organ growth; role of $\mathrm{Zn}$ homeostasis in insulin secretion/responsiveness; may stimulate food intake via orexigenic peptides secretion/responsiveness; may stimulate

Constituent of several metalloenzymes (for example, superoxide dismutase cofactor); role in amino acid, lipid and carbohydrate metabolism, insulin secretion and cholesterol synthesis; favours fat and sugar assimilation; improves cartilage elasticity and membrane quality of small blood vessels; protects vitamins (thiamin, biotin and vitamin E); role in synovial liquid formation; essential for growth and
Colon cancer; large bowel health; type 2 diabetes; CVD

Mental health (fatigue, concentration, cognitive development and reduced intellectual performances); physical health (anaemia reduced effort and resistance to infections); bone health

Mental health (fatigue, stress and anxiety); type 2 diabetes; CVD (for example, atherosclerosis and hypertension); bone health (skeletal growth and osteoporosis); nervous and muscular equilibrium; renal stones

Immunoprotection; brain and mental health; atherosclerosis; cancers (for example, oesophagus); skeletal growth and maturation; olfaction (anosmia); type 2 diabetes; weight regulation (for example, anorexia)

Anti-ageing; vascular sclerosis; bone formation (for example, osteoporosis); cancers 
reproduction; anti-carcinogenic: manganese superoxide dismutase plays a role in regulating tumour cell growth and its over-expression suppresses NF-кB activation in carcinogenic process

Component of numerous metalloenzymes acting as oxidases to achieve the reduction of molecular oxygen (for example, superoxide dismutase cofactor, lysyl oxidase or cytochrome $c$ oxidase); anti-inflammatory and anti-infectious effects; role in neurotransmission, and $\mathrm{Hb}$ and collagen fibre synthesis; reverses cardiomyocyte hypertrophy; anti-cancer effect of $\mathrm{Cu}$-DNA complexes (for example, with guanine). Role in well-balanced cholesterolaemia and glucose tolerance; antioxidant effect mainly as superoxide dismutase cofactor; low $\mathrm{Zn}$ :Cu ratio associated with reduced risk of $\mathrm{CHD}$

Glutathione peroxidase/thioredoxin reductase cofactor: protects cell membranes from lipid oxidation damage; role at the catalytic site of multiple selenoproteins; tumour growth suppressor (selective apoptotic activity according to the tissue considered); reduces susceptibility to experimental carcinogens; immune system stimulation and role in anti-infective mechanisms; helps liver to eliminate toxins; role thyroid hormone synthesis; may improve insulin resistance and protect vascular endothelium; insulin-like actions; prevents platelet aggregation

Most abundant mineral after Ca within organism ( $80 \%$ is in skeleton, $10 \%$ in muscles and $10 \%$ in nervous tissues and blood); supports tissue growth through temporary storage and transfer of energy (for example, ATP/ADP), helps in maintaining normal $\mathrm{pH}$ (acidity regulation through buffering effect) and activation of many catalytic proteins by phosphorylation; limits Ca escape and its metabolism; DNA/RNA, myelin and hydroxyapatite constituent; occurs structurally as phospholipids (for example, lecithin), a major component of most biological membranes; stimulates B-complex vitamins; may reduce the risk of colorectal tumours

Most abundant mineral within organism (about $98 \%$ in bones as hydroxyapatite); role in bone and teeth formation, cellular exchanges, nerve transmission, blood coagulation muscle contraction (for example, in heart), $\mathrm{pH}$ regulation, $\mathrm{P}$ retention and glandular secretion; essential signal transduction element (for example, cell cycle progression regulation); intracellular $\mathrm{Ca}$ is vital for regulation of cell proliferation and growth; may reduce systolic blood pressure; enzyme activation; role in vitamin $\mathrm{B}_{12}$ assimilation, blood acid-base equilibrium and clotting, $\mathrm{Fe}$ metabolism and immune system maintenance; inversely associated with type 2 diabetes; would regulate fat metabolism in adipocytes

$50 \%$ of $\mathrm{Na}$ is in extracellular liquids; generally associated with chlorine $(\mathrm{NaCl})$; role in nervous and muscular impulse transmission, in control of arterial pressure and acidity regulation; role in water repartition within organism: essential in hydroelectric equilibrium by yielding most part of extracellular liquid osmotic pressure

Cation essentially intracellular; role in acid-base equilibrium; favours $\mathrm{Na}$ excretion; inhibition of neuromuscular excitability; role in regulation of aldosterone excretion within glomerular zone; role in action of numerous enzymes; role in gastric acid and insulin secretion, and in blood pressure regulation; improves ventricular arrhythmia; may have inhibitory effects on free radical formation from macrophages and endothelial cells and on LDL oxidation, vascular smooth muscle cell proliferation (for example, neointima formation) and arterial thrombosis; reduces platelet sensitivity to thrombin; may reduce macrophage adherence to vascular wall; role in glucose control

Involved in glucose metabolism and Krebs cycle through thiamin-dependent enzymes, and in branched-chain amino acid metabolism; works to promote healthy nerves (for example, neuromodulation of chlorine canals in brain and involvement in neurotransmitter synthesis); improves mood; strengthens the heart and may restore peripheral vascular resistance; improves heartburn; antioxidant
Brain and mental health (central nervous system dysfunction); bone, tendon and cartilage health (for example, osteoarthritis); cardiovascular health (for example, IHD); cancers

Anti-ageing; CVD; immunoprotection; breast, prostate, gastrointestinal, liver, brain, skin and lung cancers; liver health; type 2 diabetes

Bone (for example, osteoporosis), teeth, mental (for example, fatigue, spasmophily, stress, memory, vigilance) and brain health; heart and kidney health digestion and growth; cancers (for example, colorectal)

Bone (for example, osteoporosis and rachitis) and teeth health; colorectal cancer; heart health (for example, hypertension and stroke); nervous system; mental health (for example, insomnia and stress); diabetes weight regulation

CVD (for example, blood pressure); nervous system hydration state

Muscular contraction (for example, cramps); hydration state; cardiovascular protection and nervous system functioning; mental health (for example, vigilance and fatigue); oedema; hypercalciura; osteoarthritis/porosis; arterial hypertension; kidney health (for example, stones); cerebro/cardiovascular diseases; type 2 diabetes

Mental (for example, Korsakoff syndrome and dry Beri Beri), neuronal (for example, neuropathy) and heart (for example, wet Beri Beri) health 
Bioactive compounds (degree of significance)

Riboflavin: $\mathrm{B}_{2}(++) \dagger$

Nicotinic acid: $\mathrm{B}_{3}(++) \dagger$

Pantothenic acid: $\mathrm{B}_{5}(++) \dagger$

Pyridoxine: $\mathrm{B}_{6}(++) \dagger$

Biotin: $B_{8}(++) \dagger$

Folate: $\mathrm{B}_{9}(+) \dagger$

Tocols: $\mathrm{E}(++) \dagger$

Tocopherols

\section{Main physiological functions and potential protective mechanisms}

Participates as a coenzyme in numerous redox reactions in metabolic pathways and energy production via the respiratory chain; assists fat, protein and carbohydrate metabolism; role in haematological status (erythrocyte formation) and gastrointestinal development; modulator of plasma homocysteine level; protects from heart tissue damage; improves skin blemishes and migraines; influence dark adaptation through riboflavin-dependent photoreceptors; participates in antioxidant defences; possibly anti-carcinogenic (for example, reduction in DNA damage)

Precursor of nicotinamide, NAD +, NAD, NADP, NADH, and NADPH: functions in many redox reactions; lowers cholesterol, LDL and NEFA levels and increases HDL level; inhibits catecholamine stimulation of lypolysis in adipose tissue (via the same post-receptor pathway as catecholamines); involved in DNA replication and repair, in cell differentiation and the production of steroid hormones in the adrenal gland

Ubiquitous vitamin; critical in the metabolism and synthesis of carbohydrates, proteins, and fats; used in the synthesis of coenzyme A, a way to transport carbon atoms within the cell (cellular respiration); involved in metabolism of fatty acids (biosynthesis), cholesterol and acetylcholine; would enhance the activity of the immune system

Needed for almost every function in the body, working as a coenzyme for numerous enzymes, notably in protein, glycogen, sphingoid bases, amino acid and fat/fatty acid metabolism; plays a major role in forming erythrocytes, neurotransmitters (such as serotonin, melatonin, dopamine and $\gamma$-aminobutyric acid) and in antibody synthesis; stabilises homocysteine levels; allows production of nicotinic acid from tryptophan; role in vitamin $\mathrm{B}_{12}$ absorption; role in humoral and cellular immunity; role in maintenance and synthesis of DNA

Functions as a coenzyme in bicarbonate-dependent carboxylation reactions (i.e. for four carboxylase enzymes such as the acetyl-CoA carboxylase): catalysis for $\mathrm{CO}_{2}$ fixation on different substrates fundamentally involved in lipid, protein and carbohydrate assimilation; necessary for cell growth and the production of fatty acids; role in the citric acid cycle; helps to maintain a steady blood sugar level; regulation of gene expression; role in normal immune function and cell proliferation (oncogene-dependent metabolic pathways)

Functions as a coenzyme in single-carbon transfers in the metabolism of nucleic and amino acids: stabilises homocysteine levels, needed to synthesise DNA bases and for DNA replication; anti-carcinogenic; prevents depletion of brain membrane phosphatidylcholine; lipotrope; effect on altered methylation and related epigenetic effects on gene expression

Strong intracellular antioxidant that protects from oxidative damages of PUFA within cell membranes and in lipoproteins, DNA nucleotidic bases and proteins; complementary effect with Se to maintain cell integrity and immune function; induction of apoptosis (anti-proliferative effect); anti-atherogenic action through, for example, reduction of oxidised LDL and of monocytes adhesion or inhibition of smooth muscle cell proliferation

Antioxidant; act directly to inhibit the formation of active carcinogenic compounds or their activation to more potent forms; possible protection of pancreatic $\beta$-cells against glucose toxicity; various non-antioxidant molecular mechanisms: inhibit protein kinase $\mathrm{C}$ (correlation with cell proliferation inhibition), produce a decrease in monocyte superoxide anion and IL-1 release and in adhesion to endothelium, effect on gene expression (possible direct action on cell signalling), and remove the suspected carcinogens peroxynitrite-derived nitrating species
Potential health protection

CVD; cancers; vision (for example, corneal opacity and cataract); mental health (for example, neurodegeneration and peripheral neuropathy); skin health

Skin health (for example, dermatitis in pellagra); lipid disorders and CVD; cancers; HIV; mental health (for example, schizophrenia, depression, insomnia); osteoarthritis

Cure of wounds, minor burns, irritations and cutaneous hurts; mental health (for example, insomnia, depression, irritability and stress); healthy digestive tract

Heart health; mental and brain health (for example, depression, fatigue, insomnia and epileptiform convulsions); colorectal cancer; asthma attacks; microcytic anaemia; occlusive arterial disease; seborrhoeic dermatitis

Mental and central nervous system health (neurologica disorders); growth; skin health (for example, dermatitis); hair health (for example, alopecia)

Neural tube defects; CVD; cancers (for example, colon); fertility; megaloblastic anaemia; mental health (cognitive impairment and depression)

Cancers (for example, pancreas); CVD; type 2 diabetes 
Tocotrioenols

Phylloquinone: $\mathrm{K}(+)$

Carotenoids

$\beta$-Carotene $(+) \dagger$

Lutein $(++)$

Zeaxanthin (+)

$\beta$-Cryptoxanthin $(+$

Polyphenols

Phenolic acid $(+++)$ (for example, ferulic acid)

Flavonoids (+) (for example anthocyanins, isoflavonoids)

Lignans $(++)$

Other compounds

Alkylresorcinols $(+++)$
Stronger antioxidant than tocopherols; role in immune response induction and bone calcification; prevent the formation of carcinogens (anti-proliferative and apoptotic inducer); cholesterol-lowering; anti-hypertensive; anti-inflammatory; anti-atherogenic; may increase bone Ca content

Functions as a coenzyme involved in the post-translational formation of $\gamma$-carboxyglutamate residues, essential for the activity of all known $\gamma$-carboxyglutamate proteins, which plays a role in coagulation (for example, vitamin K-dependent clotting factors such as prothrombin - factor II and factors VII, IX and $\mathrm{X}$ ), bone metabolism (probable role in $\mathrm{Ca}$ uptake) and vascular biology; in the intestines, assists in converting glucose to glycogen; anti-haemorrhagic factor

Antioxidant; precursor of vitamin A; tumour growth suppressor (i.e. apoptosis inducer)

Antioxidant by quenching single oxygen, neutralising photosensitisers and inhibiting lipid peroxidation; inhibits progression of aberrant crypt foci; protects ocular functions as antioxidant/optical filter and by increasing macular pigment density; reduces the skin inflammatory response; can inhibit thickening of the carotid artery walls and LDL-induced migration of monocytes to artery cell walls

Antioxidant by quenching single oxygen, neutralising photosensitisers and inhibiting lipid peroxidation; reduces the skin inflammatory response; protects ocular functions as antioxidant/optical filter and by increasing macular pigment density

Antioxidant; induces anabolic effects on bone components by increasing $\mathrm{Ca}$ content and alkaline phosphatase activity; possible protector against carcinogenesis (for example, bioregulatory function in the control of cell differentiation and apoptosis); precursor of vitamin A, retinal and retinoic acid

Antioxidant (for example, scavenges superoxide anion radical and reduces oxidative stress caused during diabetes, for example, lipid peroxidation); anti-apoptotic; antimicrobial; anti-inflammatory; blood cholesterol- and glucose-lowering; UV absorber; interferes with intracellular signalling pathways; hypotensive effect by reducing blood pressure (vascular relaxation); tumour growth suppressor; enzyme modulator; may increase ( $\beta$-cell mass; may decrease adipose tissues, serum lipid profiles, insulin and leptin

Antioxidant (for example, against LDL-cholesterol oxidation); tumour growth suppressor; enzyme modulator; role in redox cell signalling, glutathione synthesis regulation and gene regulation; modulate angiogenesis; anti-microbial and antiinflammatory; inhibition of platelet aggregation (for example, may affect arachidonic acid metabolism through inhibition of lipoxygenase activity); stimulation of uric acid production; role in fat oxidation and decreased adipose tissues; may decrease genistein may improve trabecular connectivity and trabecular thickness

Antioxidant: may reduce fatty acid oxidation; precursors of enterolactone and enterodiol; anti-carcinogenic activity: inhibit cell proliferation by competing with oestradiol for nuclear type II oestrogen-binding sites, stimulation of differentiation, may inhibit the tyrosine-specific protein kinase (associated with cellular receptors for several growth factors) and DNA topoisomerase, prevent the production of oestrone from androstenedione, and influence cholesterol homeostasis (for example, deoxycholic acid is correlated with increased colon cancer); anti-atherosclerotic; hypolipidaemic effect (improves blood lipid profile); diuretic action and antagonistic action of platelet-activating factor receptor; priming action on superoxide production on human neutrophiles; anti-bacterial and anti-fungal; may inhibit bone resorption

Antioxidant (for example, modulator of lipid oxidation); anti-microbial, anti-parasitic and anti-carcinogenic properties; inhibitors of 3-phosphoglycerate dehydrogenase (key enzyme of TAG synthesis in adipocytes); direct effect on structure and metabolism of nucleic acids; can be incorporated into biological membranes (for example,
Neurodegeneration; cancers; CVD; type 2 diabetes osteoporosis; obesity

Bone health (osteoporosis and bone loss); atherosclerosis haemorrhagic event and haematuria (reflects tumours or kidney stones?)

Colon cancer; lung cancer in non-smokers; coronary artery disease

Visual function protection (for example, age-related macular degeneration, cataract and glaucoma); stroke and atherosclerosis; lung and colon cancer; skin health

CVD (for example, stroke); visual function protection (for example, cataract); skin health; lung cancer

Bone loss; lung cancer; visual function

Cancer; CVD (for example, thrombosis and atherosclerosis); neurodegenerative disorders (for example, Alzheimer's and Parkinson's diseases); type 2 diabetes; skin health; anti-ageing; hepatoprotective; pulmonary protective; hypertension; obesity

Cancer; CVD; body-weight regulation/obesity; type 2 diabetes; bone development and osteoporosis

Colon cancer; hormonally mediated diseases (for example, breast and prostate cancers); CVD (for example, stroke); osteoporosis and rheumatoid arthritis; gastric and duodenal ulcers; skin health

Cancer; tuberculosis; tropical diseases 


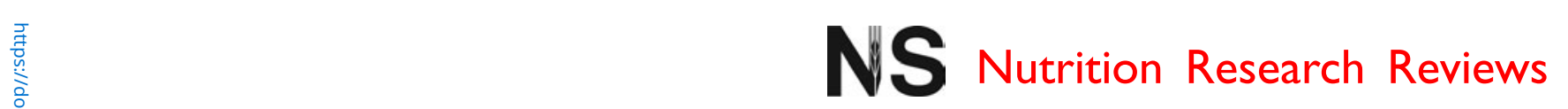

Table 3. Continued

Bioactive compounds (degree of significance)

Betaine $(+++)$

Choline $(++)$

Phytosterols $(++)$ (for example, $\beta$-sitosterol)

Inositols $(++)$ (for example myo- and chiro-inositol, pinitol)

Policosanol $(++)$ (for example, octacosanol)
Main physiological functions and potential protective mechanisms

Potential health protection

modulate phospholipid bilayer properties by inhibiting the activity of some membrane-bound enzymes); cholesterol-lowering in liver

Osmoprotectant; methyl donor: increases DNA methylation and decreases hyperhomocysteinaemia; role in sulfur amino acid homeostasis; antioxidant; able to reverse insulin resistance; inversely associated with serum non-HDL-cholesterol, TAG, BMI, percentage body fat, systolic and diastolic blood pressure, and positively associated with HDL-cholesterol; inversely associated with inflammatory markers related to atherosclerosis (C-reactive protein and TNF- $\alpha$ ); inverse association with colorectal adenoma; lipotrope

Precursor of betaine, acetylcholine (neurotransmitter), membrane phospholipids (phosphatidylcholine and sphingomyelin: role in intracellular signalling) and plateletactivating factor (potent messenger molecule); methyl donor (for example, DNA methylation and reduced hyperhomocysteinaemia); epigenetic regulator of gene expression; promotes carnitine conservation (for example, accretion in skeletal muscle); antioxidant-type action; lipotrope: role in lipid metabolism (for example, increases fatty acid oxidation), and in integrity and signalling functions of cell membranes; accelerates the synthesis and release of acetylcholine

Lower serum total and LDL-cholesterol: compete with cholesterol for micelle formation in the intestinal lumen (and increase its excretion) and inhibit dietary and biliary cholesterol absorption; anti-inflammatory; may protect from vascular smooth muscle cell hyperproliferation; effect on immune system (for example, may prevent immunosuppression associated with excessive physical stress, i.e. immunemodulatory activity on human lymphocytes); $\beta$-sitosterol inhibits carcinogen-induced neoplasia (suppressing agent) and might mediate apoptosis through caspase activation; hypoglycaemic; anti-pyretic

Involved in several biological processes as secondary messenger molecules: in insulin signal transduction, cytoskeleton assembly, nerve guidance, intracellular $\mathrm{Ca}$ concentration control, gene expression and breakdown of fat and reducing of blood cholesterol; myo-inositol may be converted into chiro-inositol in vivo (epimerisation) and is precursor for several phospholipids (for example, phosphatidylinositol 4-phosphate) playing a role in membrane structure and function; chiro-inositol improves insulin resistance and helps in controlling blood glucose, ovulatory functions (i.e. increased ovulation), decreases serum androgen and plasma TAG concentrations, and reduces blood pressure; myo-inositol depresses the rise in TAG and total lipid liver, hepatic activities of glucose-6phosphate dehydrogenase, malic enzyme, fatty acid synthetase and citrate cleavage enzyme; free inositol is involved in volume regulation during persistent osmotic stress; reduces myelinolysis after rapid correction of chronic hyponatraeosmotic stress; reduces myelinolysis after rapid correction of chronic hyponatrae-
mia; may reduce mammary and colon carcinoma; impaired myo-inositol metabolism mia; may reduce mammary and colon carcinoma; impaired myo-inositol metabolism would be linked to altered nerve
impaired sciatic nerve Na-K ATP

Antioxidant by reducing LDL and membrane lipid peroxidation; decreases platelet aggregation, endothelial damage and foam cell formation; vasodilatating effect; cholesterol-lowering by inhibiting cholesterol synthesis at the earliest step of the biosynthetic pathway (through down-regulating 3-hydroxy-3-methyl-glutaryl CoA reductase); lowers plasma LDL-cholesterol and increases plasma HDL-cholesterol level; affects lipid metabolism; prevents smooth muscle cell proliferation; role in cytoprotection; active energy-releasing factor; affects the nervous system (for example, anti-fatigue and improves reaction time to a visual stimulus)
CVD; liver and kidney health; colorectal cancer; athletic performances; type 2 diabetes; metabolic syndrome

Brain development and normal learning and memory functions; weight regulation; fetal development (for example, neural tube); liver dysfunctions (for example, fatty liver); cancer; CVD

CVD; colon, breast and benign prostate cancer; type 2 diabetes

Type 2 diabetes (for example, diabetic polyneuropathy) polycystic ovary syndrome or compromised fertility (for example, insulin resistance hyperandrogenism and oligo-amenorrhoea); neural tube defects; CVD; neurological and psychiatric diseases (for example, bipola depression, panic attacks and obsessive-compulsive disorders); severity of osmotic demyelination syndrome; cancers; intestinal lipodystrophy

CVD (for example, atherosclerosis and hypertension) gastric ulcer; athletic performances; mental health 
Melatonin (+)

para-Aminobenzoic acid

Specific cereal compounds $\gamma$-Oryzanol in rice

Avenanthramides in oats

Saponins in oatsł

$\beta$-Glucans in oats and barley
Antioxidant (but irreversibly oxidised), the most potent physiological scavenger of $\mathrm{OH}$ : for example, reverses massive DNA degradation, stimulates glutathione peroxidase activity, increases gene expression for antioxidant or may protect against lenticular protein oxidation enzymes; effects on mood, happiness, sleep-wake period regulation and brain neuromodulation; promotes mitochondrial respiration; anticarcinogenic, anti-proliferative and oncostatic effects through antioxidant, immunostimulating and apoptotic properties (for example, increase of natural killer cell activity as well as the stimulation of cytokine production), and also effects on gene expression; regulates the glucocorticoid re the activation of oestrogen receptor for DNA binding

Role in folate formation: for example, stimulates bacterial growth within intestines, enabling them to produce folates; cholesterol-lowering; anti-carcinogenic through down-regulation of $\mathrm{N}$-acetyltransferase which may activate human carcinogens; acetylation in blood, notably by platelets: arachidonic acid is a main acetyl donor, suggesting the involvement of peroxisomal $\beta$-oxidation; anti-aggregatory effect: inhibits the production of thromboxane, which participates in increased arterial pressure through vasoconstriction and in blood coagulation, in human platelets; chromotrichial effect on grey hair

Antioxidant (for example, decreases serum lipid peroxides); lowers total and LDLcholesterol and increases HDL-cholesterol through its tocotrienol and fibre contents; improves glycaemia control through its lipoic acid content: increases glucose uptake by insulin-resistant muscle to produce energy; anti-ulcerogenic; inhibition of platelet aggregation; stimulates hypothalamus (link between nervous and endocrine system), for example, change in serum growth hormone level

Antioxidant, anti-inflammatory and anti-atherogenic: inhibit smooth muscle cell proliferation and increase NO production, inhibit aortic endothelial cell expression of adhesion molecules and their adhesion to monocytes, and reduce production of several pro-inflammatory cytokines and chemokines

Antioxidant (for example, activate transcriptional activity of $\mathrm{Cu}$, Zn-superoxide dismutase gene, scavenge superoxides and reduce lipid peroxidation); cause hypoglycaemia and hypoinsulinaemia; reduce non-enzymic protein glycation (i.e. $\mathrm{HbA}_{1 \mathrm{c}}$ level); partially reverse hypercholesterolaemia (by binding with cholesterol and impairing its absorption and/or by binding bile acids, by interfering with their enterohepatic circulation and by increasing their faecal excretion) and hypertriacylglycerolaemia; anti-fungal and anti-viral; immunostimulant and anti-carcinogenic ( system functioning (for exar junction communication)

Plasma cholesterol- and glucose-lowering; may indirectly affect the metabolism of bile acids (i.e. formation of secondary bile acids) and neutral sterols in intestine and liver; anti-mutagenic; anti-microbial; anti-parasitic; stimulate immune functions (for example, may stimulate proliferation and activation of peripheral blood monocytes)
Mental and brain health (for example, Alzheimer's disease, depression and sleep troubles); anti-ageing; cancers (for example, colorectal, breast and prostate); cataract

CVD (for example, atherosclerosis and hypertension); skin (for example, UV absorber and vitiligo) and hair health; collagen diseases; leukaemia; rheumatic fever and rickettsial diseases

Climacteric disturbances (i.e. menopausal troubles) and autonomic nervous imbalance (autonomic ataxia); type 2 diabetes; CVD; gut health (for example, gastric ulcer) mental health (for example, anxiety)

Atherosclerosis

Colon, breast, prostate and skin cancers; CHD; skin health; nervous system health (for example, harmful stress on organs); liver health; type 2 diabetes

Cancers; CVD; type 2 diabetes; gut health

* All data concerning physiological mechanisms and health effects are based on international references (in vitro studies on culture cells and in vivo studies in animals and human subjects; see references in Appendices). $\dagger$ For these compounds, the intensity of the symbol in brackets $(+,++$ or +++$)$ refers to the importance of the compound as supplied by a predominantly cereal-based diet, based on British data collected by Truswell ${ }^{(19)}$; for other compounds, the intensity of the symbol in brackets was estimated based on the compound content in whole-grain wheat compared with other food sources.

¥ Mechanisms and health outcomes are associated with plant saponins in general, not exclusively cereal saponins. 
Table 4. Whole-grain cereal bioactive compounds potentially involved in the prevention of major health outcomes and in antioxidant protection*

\begin{tabular}{|c|c|}
\hline Major health outcome & Bioactive compound \\
\hline Body-weight regulation and obesity & $\begin{array}{l}\text { Insoluble fibre, fructans, resistant starch, } \mathrm{Zn}, \mathrm{Ca} \text {, tocotrienols, phenolic acids, flavonoids, } \\
\text { choline, } p \text {-aminobenzoic acid }\end{array}$ \\
\hline CVD and heart health & $\begin{array}{l}\alpha \text {-Linolenic acid, methionine, oligosaccharides, soluble fibre, resistant starch, } \\
\text { phytic acid, Mg, Mn, Cu, Se, K, thiamin, riboflavin, nicotinic acid, pyridoxine, folates, } \\
\text { tocopherols, tocotrienols, phylloquinone, } \beta \text {-carotene, lutein, zeaxanthin, } \\
\text { phenolic acids, flavonoids, lignans, phytosterols, betaine, choline, inositols, } \\
\text { policosanol, } p \text {-aminobenzoic acid, } \gamma \text {-oryzanol, avenanthramides, saponins }\end{array}$ \\
\hline Type 2 diabetes & $\begin{array}{l}\text { Soluble fibre, resistant starch, phytic acid, Mg, Zn, Se, K, Ca, tocopherols, tocotrienols, } \\
\text { phenolic acids, flavonoids, betaine, inositols, phytosterols, } \gamma \text {-oryzanol, saponins }\end{array}$ \\
\hline Cancers & $\begin{array}{l}\alpha \text {-Linolenic acid, oligosaccharides, soluble fibre, insoluble fibre, resistant starch, lignin, } \\
\text { phytic acid, Zn, Mn, Cu, Se, P, Ca, riboflavin, nicotinic acid, pyridoxine, folates, } \\
\text { tocopherols, tocotrienols, } \beta \text {-carotene, } \beta \text {-cryptoxanthin, phenolic acids, flavonoids, } \\
\text { lignans, alkylresorcinols, betaine, choline, inositols, phytosterols, melatonin, } \\
\text { p-aminobenzoic acid, saponins }\end{array}$ \\
\hline Gut health & $\begin{array}{l}\alpha \text {-Linolenic acid, oligosaccharides, soluble fibre, insoluble fibre, resistant starch, } \\
\text { riboflavin, pantothenic acid, phenolic acids, policosanol, } \gamma \text {-oryzanol }\end{array}$ \\
\hline $\begin{array}{l}\text { Mental/brain/nervous system health and } \\
\text { neurodegenerative disorders }\end{array}$ & $\begin{array}{l}\alpha \text {-Linolenic acid, methionine, oligosaccharides, } \mathrm{Fe}, \mathrm{Mg}, \mathrm{Zn}, \mathrm{Cu}, \mathrm{P}, \mathrm{Ca}, \mathrm{Na}, \mathrm{K} \text {, } \\
\text { thiamin, riboflavin, nicotinic acid, pantothenic acid, pyridoxine, biotin, folates, } \\
\text { tocotrienols, phenolic acids, choline, inositols, policosanol, melatonin, } \\
\gamma \text {-oryzanol, saponins }\end{array}$ \\
\hline $\begin{array}{l}\text { Skeleton health (i.e. bone, tendon, cartilage, } \\
\text { collagen, articulation and teeth) }\end{array}$ & $\begin{array}{l}\alpha \text {-Linolenic acid, Fe, Mg, Zn, Mn, Cu, P, Ca, K, nicotinic acid, tocotrienols, phylloquinone, } \\
\beta \text {-cryptoxanthin, flavonoids, lignans, } p \text {-aminobenzoic acid }\end{array}$ \\
\hline $\begin{array}{l}\text { Antioxidant protection (development of diseases } \\
\text { in relation to increased oxidative stress) }\end{array}$ & $\begin{array}{l}\text { Reduced glutathione, methionine, cystine, lignins, phytic acid, } \mathrm{Mg}, \mathrm{Fe}, \mathrm{Zn}, \mathrm{Mn}, \mathrm{Cu} \text {, } \\
\text { Se, thiamin, riboflavin, tocopherols, tocotrienols, } \beta \text {-carotene, lutein, } \\
\text { zeaxanthin, } \beta \text {-cryptoxanthin, phenolic acids, flavonoids, lignans, } \\
\text { alkylresorcinols, betaine, choline, policosanol, melatonin, } \gamma \text {-oryzanol, } \\
\text { avenanthramides, saponins }\end{array}$ \\
\hline
\end{tabular}

* Prepared from data in Table 3.

a whole-grain product. The issue is still debated. The definition given by the American Food and Drug Administration ${ }^{(26)}$ in 1999 was: 'For purposes of bearing the prospective claim, the notification defined 'whole grain foods' as foods that contain 51 percent of total weight or more whole grain ingredient(s) by weight' (extract). This definition was debated and contested by the European Whole Grain Task Force in 2008. They explained that: 'Using total weight gives advantage to products sold by dry weight such as crackers and ready-to-eat cereal. Because foods like breads have a proportionally high water content, even some breads made with all whole grain flours but containing significant amounts of nuts, seeds and fruit would fail to meet the $51 \%$ by weight rule ${ }^{\text {(24) }}$. Apparently, there is still no international consensus as to the right proportion of whole grain by dry weight (DW) in a product in order for it to be called a whole-grain product. Each country has its own definition and standards ${ }^{(21)}$. However, most research and observational studies, particularly those on breakfast cereals, estimate the whole-grain intake from products containing at least $25 \%$ whole grains or bran by weight ${ }^{(5,14,27,28)}$. Thus, a study on young individuals aged 4-18 years found that using a $51 \%$-based definition underestimated the whole-grain intake by $28 \%$, breakfast cereals $(56 \%)$ and bread $(25 \%)$ being the major sources of whole-grain cereals ${ }^{(29)}$. In another study on adiposity among two cohorts of British adults, the same research team assumed that whole-grain foods contained $\geq 10 \%$ whole grains and found little or no association between the wholegrain intake and anthropometric indices ${ }^{(30)}$. This suggests that the threshold of $10 \%$ is probably too low and emphasises the need to harmonise how the whole-grain cereal food intake is calculated. In these studies, generally carried out in Western countries, whole-grain cereal foods considered are, for the most cited, whole-grain breads (for example, dark, brown, wholemeal and rye bread), wholegrain breakfast cereals (for example, muesli), popcorn, cooked porridges (oatmeal or whole wheat), wheat germ, brown rice, bran, cooked grains (for example, wheat, millet and roasted buckwheat) and other grain-based foods such as bulgur and couscous. A complete list of food ingredients classified as whole grains in the US Department of Agriculture (USDA) pyramid servings database is reported by Cleveland et al. ${ }^{(31)}$. Refined grain foods generally include white breads (for example, French baguette), sweet rolls, noodles, pasta, cakes, biscuits, viennoiseries, muffins, refined grain breakfast cereals, white rice, pancakes, waffles and pizza.

\section{The importance of whole-grain cereal product consumption}

There are far fewer whole-grain cereal products on the market than there are refined products, at least in Western countries. The major sources of whole-grain cereals are breads, breakfast cereals and whole-grain cereals consumed as such (for example, brown rice or quick-cooking wholegrain barley and wheat). Epidemiological data show that the consumption of two to three servings of whole-grain cereal per $d$ is sufficient to get beneficial health effects ${ }^{(32)}$. The recommended consumption of whole-grain cereal products differs from one country to another, but most recommend increased whole-grain cereal product consumption ${ }^{(21,32)}$. For example, at least three servings daily are recommended in the USA, that is, about $48 \mathrm{~g}$ of whole-grain cereals ${ }^{(33)}$; between six and twelve servings daily are recommended in Australia and four servings daily in Denmark ${ }^{(21)}$. 
Other countries such as Canada, UK, Greece, Germany, Austria and Switzerland are not so precise and generally recommend an increase in cereal consumption with emphasis on whole-grain products ${ }^{(21)}$. Surveys carried out in the USA and the UK showed that most individuals consume less than one serving per $\mathrm{d}$ and about $30 \%$ any, and that only 0.8 to $8 \%$ of those surveyed in the USA consumed the recommended three servings per $\mathrm{d}^{(31,32,34)}$. The situation is quite different in Scandinavian countries, where individuals consume more whole-grain cereal products, particularly rye-based ${ }^{(32)}$. For example, Norwegians consume an estimated four times more whole-grain products than do Americans ${ }^{(35)}$, but less than the Finns, $40 \%$ of whom may consume four or more slices of dark bread per $\mathrm{d}^{(36)}$. Why is consumption so low in other Western countries? There are probably several reasons. First, unlike fruits and vegetables, individuals do not know about the benefits of whole-grain cereal products. Second, individuals tend to think that whole-grain cereal products are not very tasty. And third, whole-grain cereal products are less common and many are difficult to identify as being wholegrain (problem of labelling). Last, time and money have been cited as obstacles to eating more nutritiously ${ }^{(37)}$.

\section{Whole-grain and wholemeal}

The terms 'whole-grain' and 'wholemeal' are mostly used synonymously. It is generally believed that whole-grain products are made with wholemeal flour, and that they may secondarily also contain intact grains. But the form in which grain is incorporated into food, intact or milled, is nutritionally significant. Thus 'wholemeal' (made of milled whole-grain flour) and 'whole-grain' (made with intact cereal grains) breads have different effects on postprandial glycaemia. The whole-grain breads produce a significantly lower glycaemic response than the wholemeal breads ${ }^{(38)}$. This underlines the importance of food structure on physiology. Thus, for clarity, the term 'whole-grain' should be used for cereal products containing more or less intact cereal kernels, and 'wholemeal' for cereal products made of more or less refined flour, in which bran, germ and endosperm are first separated, and then reassembled, in proportions that rarely correspond to those of intact grains, as the germ fraction is generally removed.

\section{Current hypotheses and mechanisms for the protective action of whole-grain cereals}

The mechanisms underlying the health benefits of wholegrain cereals are undoubtedly multi-factorial. A recent cross-sectional study on 938 healthy men and women showed that a higher consumption of whole grains, bran and germ was associated with a significant decrease in plasma homocysteine (hyperhomocysteinaemia is a risk factor for CVD) and of some markers of blood glucose control, inflammation and lipid status ${ }^{(17)}$. Other studies have linked the consumption of high-whole-grain diets with improved BMI and insulin sensitivity, lower concentrations of serum TAG, total and LDL-cholesterol and inflammation markers, and higher plasma or serum enterolactone ${ }^{(2,39-42)}$. Except for enterolactone, for which high serum levels are associated with reduced risk of $\mathrm{CVD}^{(43)}$, all of the other biomarkers, when outside a normal healthy range, are all risk factors associated with the development of diabetes and CVD. There is the same kind of significant negative association between whole-grain consumption and the risk of digestive cancer $^{(44,45)}$. Other mechanisms are involved in this, including the capacity of several whole-grain compounds to suppress tumour growth ${ }^{(46)}$. The next section describes the main known mechanisms by which whole-grain cereals help protect the gut and prevent the development of obesity, diabetes, CVD and cancers.

\section{Food structure}

The structure of food has long been recognised as an important parameter governing the health benefit of wholegrain cereal products. The first study was performed in 1977 by Haber et $\mathrm{al}$. on the influence of apple structure (intact apples $v$. apple purée $v$. fibre-free apple juice) on satiety, plasma glucose and serum insulin. The removal of fibre and/or the disruption of the physical food structure was accompanied by reduced satiety, disturbed glucose homeostasis and an inappropriate insulin response ${ }^{(47)}$. Almost 10 years later, it was shown that simply swallowing carbohydrate-rich foods (rice, apple, potato and sweetcorn) without chewing was sufficient to significantly decrease postprandial glycaemia ${ }^{(48)}$. This was the simplest way to emphasise the importance of food structure (chewing $v$. no chewing) on digestion. Then, Jenkins et al. studied the effects of wholemeal and wholegrain breads and showed that the glycaemic index (GI) of wholemeal breads (wheat or barley flour-based) without intact grains was the same as that of white bread made of refined flour $(>90)$, and that increasing the intact barley kernel or cracked wheat grain content of the bread (50 and $75 \%$ ) resulted in a significantly large decrease in the GI from $92-96$ to $39^{(38)}$. Thus, an intact botanical food structure is more important than the composition of the food (the presence of fibre in wholemeal bread and absence from white bread) for influencing physiological responses like those related to satiety and glucose metabolism. Many later studies have confirmed these results, emphasising the importance of preserving the natural initial fibrous network, particularly in more or less intact wheat, barley, rye and oat kernels ${ }^{(49-52)}$.

\section{Whole-grain cereals as a rich source of fibre}

Dietary fibre is defined by the AACC as 'the edible parts of plants or analogous carbohydrates that are resistant to digestion and absorption in the human small intestine with complete or partial fermentation in the large intestine. Dietary fibre includes polysaccharides, oligosaccharides, lignin and associated plant substances. It promotes beneficial physiological effects including laxation and/or blood cholesterol attenuation and/or blood glucose attenuation' ${ }^{(53)}$. This definition includes that fraction of starch not digested in the small intestine, resistant starch (RS). Wholegrain wheat may contain from 9 to $17 \mathrm{~g}$ total fibre per $100 \mathrm{~g}$ edible portion (Table 2), which is more than in most vegetables (generally $<6 \mathrm{~g} / 100 \mathrm{~g}$ edible portion). Thus, consuming whole-grain cereal products is undoubtedly 
a good way of increasing the fibre intake from the $10-15 \mathrm{~g} / \mathrm{d}$ eaten by most Western populations to the recommended level of about $30-35 \mathrm{~g} / \mathrm{d}$.

Wheat is relatively poor in soluble fibre. It has been found that the soluble:insoluble fibre ratio is about 1:5 for wholegrain wheat, 1:10 for wheat bran and 1:3 for wheat germ (Table 2). Whole-grain wheat therefore provides large quantities of insoluble fibre (up to $11 \mathrm{~g} / 100 \mathrm{~g}$ ) and RS (up to $22 \%$ for certain high-amylose barley varieties ${ }^{(54)}$ ). Cereal fibre is now recognised to be beneficial for bowel health. Wheat has a great diversity of fermentable carbohydrates. Except for lignin, whose nutritional benefits are not really known, all the types of fibre compounds, including soluble and insoluble fibre, oligosaccharides and RS, have important physiological properties and provide significant health benefits $^{(18,55)}$. For example, soluble fibre increases viscosity, which delays gastric emptying and limits glucose diffusion towards the enterocytes for absorption. This leads to a lower glucose response when sufficient quantities are ingested ${ }^{(56)}$.

Cereal fibres also increase satiety and help control body weight ${ }^{(57)}$. The mechanisms by which dietary fibre positively affect body weight have been previously described: briefly, they involve hormonal effects via reduction of the insulin secretion, metabolic effects via increased fat oxidation and decreased fat storage due to greater satiety, and colonic effects via SCFA production ${ }^{(58)}$. Thus, the consumption of highly viscous fibre such as $\beta$-glucans, found mainly in barley and oats, is now recommended for the management of glucose homeostasis in type 2 diabetic subjects ${ }^{(59)}$. Soluble fibre has also been shown to reduce cholesterolaemia in ileostomy subjects ${ }^{(60)}$ by probably favouring an increase in bile acid excretion as shown in ileostomates following oat $\beta$-glucans consumption ${ }^{(61)}$. Increased bile acid excretion stimulates bile acid synthesis from serum cholesterol, so reducing cholesterolaemia ${ }^{(61)}$.

The fermentation of fibre and RS within the colon produces SCFA that are associated with a lower risk of cancer $^{(62,63)}$, favouring the development of a healthy colonic microbiota (i.e. prebiotic effect) ${ }^{(64)}$. These SCFA also reduce the proliferation of human colon cancer cell lines in vitro ${ }^{(62,63)}$. RS is known to produce large quantities of butyrate $^{(65)}$. The increased butyrate production by rats fed wheat bran is negatively associated with the proliferation of colon crypt cells that are involved in the development of colorectal cancer ${ }^{(66)}$. RS also significantly increases fat oxidation in humans, probably by increased SCFA production that inhibits glycolysis in the liver, so rendering it more dependent on fat-derived acetyl CoA as fuel, this effect being associated with a concomitant decrease in carbohydrate oxidation and fat storage ${ }^{(67)}$.

In contrast, insoluble fibre, which is poorly fermented in the colon, favours an increased transit time and greater faecal bulking ${ }^{(68)}$, two parameters that probably prevent colon cancer by diluting carcinogens and reducing their time in contact with epithelial cells ${ }^{(69)}$. The fermentation of some fibre also increases mineral absorption in rats, mainly by increasing the surface area available for absorption (epithelial cell hypertrophy) and/or by favouring better hydrolysis of phytic acid via enhanced fermentation, as was shown with $\mathrm{RS}^{(70,71)}$ and inulin (a fructan-type compound) ${ }^{(72,73)}$.

\section{Whole-grain cereals and butyrate production}

Whole-grain cereal products are an important indirect source of butyrate, produced notably through RS fermentation ${ }^{(65)}$. Butyrate has cancer-preventing properties in rats by inducing apoptosis ${ }^{(74)}$ or reducing tumour mass ${ }^{(75)}$. But its positive physiological action may not be restricted to these two effects. The precise mechanisms involved in the anticolon cancer effect of butyrate have been reviewed from in vitro, animal and human studies and they mainly include a combination of several physiological modifications in relation to abnormal cell growth inhibition, immune system stimulation and modulation of DNA repair and synthesis ${ }^{(65)}$. Butyrate might also protect against breast and prostate cancers, as shown by in vitro studies on mammary ${ }^{(76)}$ and prostate $^{(77)}$ cancer cell lines ${ }^{(65)}$. The RS content of wholegrain cereal products depends on the proportion of the different types of RS: RS1 which is physically inaccessible to $\alpha$-amylase, RS2 which is raw starch granules, and RS3 which is recrystallised/retrograded amylose that is formed when cooked food cools. It is therefore difficult to obtain precise data on the RS content of whole-grain cereal products, but some products are enriched in RS by selecting high-amylose varieties of cereal. Nevertheless, products containing whole grains or made from high-amylose cereal varieties will have proportionally higher RS contents and produce more butyrate, as was shown in human subjects fed various breads, breakfast cereals and crackers ${ }^{(78,79)}$. Wholegrain cereal products with an intact botanical structure, that is with intact kernels, will have a higher RS1 content, since it is inaccessible to $\alpha$-amylase, and butyrate production. The relationship between the consumption of whole-grain cereals and/or their bran and germ fractions, butyrate production and long-term health effects deserve to be studied more thoroughly in human subjects, particularly because of the effects in rats of butyrate on fat oxidation and of total SCFA production on cholesterol synthesis reduction $^{(80)}$.

\section{The 'second-meal effect'}

The 'second-meal effect' is characterised by an improved carbohydrate tolerance at a meal (either lunch or breakfast, called the 'second meal') about 4-5 or 10-12 h after the consumption of a low-GI meal (i.e. the 'first meal'), an effect which may contribute to the long-term metabolic benefits of low-GI diets. It was first described by Jenkins et al. who used viscous guar gum ${ }^{(81)}$, and thereafter for lowGI carbohydrate foods such as lentils ${ }^{(82)}$. Recently, mechanisms have been proposed to explain the sustained positive effect of low-GI whole-grain products composed of intact barley or rye kernels consumed at diner or breakfast on the glycaemic response at the following meal, breakfast or lunch ${ }^{52,54,83)}$.

The physiological mechanisms involved appear to differ according to the interval between the two meals, dinner to breakfast (about 10-12h) or breakfast to lunch (about $4-5 \mathrm{~h}$ ). The shorter period seems to be sufficient for the low-GI feature of the cereal product consumed at breakfast to reduce the glucose response at lunch, probably by improving blood sugar regulation and insulin sensitivity ${ }^{(54)}$. 
The longer interval between dinner and breakfast involved the fermentation of indigestible carbohydrates in the colon, reduced plasma NEFA and modified glucose metabolism. This indicates that the presence of specific dietary fibre (soluble or insoluble or RS) in boiled barley kernels is more significant in this 'second-meal effect' than is its low GI.

SCFA produced during the fermentation of fibre in the colon might be particularly involved ${ }^{(83)}$ through at least three potential processes: a possible decrease of the gastric emptying rate by SCFA as reviewed in rats and humans ${ }^{(84)}$, notably through an increased level of the polypeptide YY in blood by SCFA, that may lead to a reduced rate of glucose entry into the bloodstream; the ability of propionate and acetate to reduce serum NEFA in humans ${ }^{85)}$, circulating fatty acids being able to induce peripheral and hepatic insulin resistance in humans ${ }^{(86)}$; and, finally, the possible specific action of propionate on glucose metabolism by increasing hepatic glycolysis and decreasing hepatic glucose production as shown in isolated rat hepatocytes ${ }^{(87)}$. A later study on healthy subjects ${ }^{(54)}$ confirmed that the lowGI feature of the products consumed in the evening meal was not per se involved in the improved glucose response at breakfast, and that the lower plasma NEFA concentration combined with the high plasma propionate content (from fermentation in the colon) contributed to the overnight benefits in terms of glucose tolerance ${ }^{(83)}$. The quantity and quality of the indigestible carbohydrates (for example, barley fibre and RS) are most important. There is also an important relationship between gut microbial metabolism and insulin resistance ${ }^{(54)}$.

These results suggest that the influence of carbohydrates on glucose tolerance over a longer time (semi-acute) is optimal when the food structure is preserved (i.e. a low-GI feature) and content of RS and/or fibre is high (i.e. production of specific SCFA). Eating barley or rye kernels for breakfast resulted in lower cumulative postprandial increases in blood glucose after breakfast, lunch and dinner (a total of $9.5 \mathrm{~h}$ ) than did a breakfast of white-wheat bread $^{(52)}$. From a technological point of view, the quantity and quality of the indigestible carbohydrates is therefore particularly important, in addition to preserving a more or less intact botanical food structure, for a better control of glucose metabolism, especially to prevent type 2 diabetes.

\section{Whole-grain cereals as rich sources of anti-carcinogenic compounds}

A survey of 61433 women found that a high consumption of whole grains (hard whole-grain rye bread, soft whole-grain bread, porridge, and cold breakfast cereals) was associated with a lower risk of colon cancer ${ }^{(11)}$. An inverse association between cereal fibre and whole-grain cereal consumption and small-intestinal cancer incidence has also been reported $^{(12)}$. The roles played by dietary fibre and phytochemicals in preventing intestinal cancer in humans and animals have been reviewed and discussed for both human intervention and animal studies ${ }^{(45,69,88)}$. The positive action of the wheat bran oil on colon tumour incidence in rats (azoxymethane-induced cancer) ${ }^{(89)}$ and mice (Min cancer model $)^{(90)}$ has also been demonstrated. This anti-carcinogenic effect is mainly attributed to the antioxidant and anti-inflammatory properties of several bioactive compounds, as increased oxidative stress and inflammation are involved in cancer aetiology ${ }^{(91)}$. Phenolic acids, flavonoids, carotenoids, vitamin E, $n$-3 fatty acids, lignan phyto-oestrogens, steroid saponins (found mainly in oats), phytic acid and Se are all potential suppressors of tumour growth, but human, animal and/or in vitro cell studies indicate that their mechanisms of action may differ (Tables 3 and 4) ${ }^{(46,69,92-95)}$. For example, cereal lignans are converted by fermentation into mammalian lignans or phyto-oestrogens (enterodiol and enterolactone). These may have a weak oestrogenic activity, and may protect against hormone-dependent cancers (prostate and breast cancers) and/or colon cancer ${ }^{(96)}$. Studies on postmenopausal women, ovariectomised rats and liver and breast cancer cell cultures indicate that phyto-oestrogens inhibit cell proliferation by competing with oestradiol for type II oestrogen binding sites ${ }^{(97,98)}$. Phytic acid would help reduce the rate of cell proliferation during the initiation and post-initiation stages (for example, decreased incidence of aberrant colon crypt foci) by complex mechanisms that involve its antioxidant properties, signal transduction pathways, gene regulation and immune response through enhancing the activity of natural killer cells ${ }^{(99)}$, and its anticarcinogenic effect seems to be dose-dependent ${ }^{(100)}$. The high phytic acid content of whole-grain cereals (up to $6 \%$ in wheat bran) has led to questions about whether the anticancer activity of wheat bran should be attributed more to phytic acid than to dietary fibre ${ }^{(69,92)}$. Indeed, pure phytic acid is more efficient at reducing the incidence and multiplicity of mammary tumours in rats than is the bran fraction (All Bran; Kellogg $\left.{ }^{\circledR}\right)^{(101)}$. The many anti-carcinogenic actions of flavonoids include their ability to inhibit various stages of tumour development in animals ${ }^{(102)}$ and to reduce the mutagenicity of several dietary carcinogens in Salmonella typhimurium TA98NR ${ }^{(103)}$. The anti-carcinogenic activity of ferulic acid is mainly attributed to its antioxidant capacity; it scavenges the free oxidative radicals that are involved in the aetiology of cancer, and to its ability to stimulate cytoprotective enzymes ${ }^{(104,105)}$. Studies on azoxymethane-treated rats indicate that vitamin $\mathrm{E}$ and $\beta$-carotene inhibit the progression of aberrant crypt foci to colon cancer, especially the later stages of carcinogenesis, while wheat bran is better at inhibiting earlier stages ${ }^{(106)}$. Lignins, by hydrophobically binding bile salts, might reduce the formation of carcinogens from them ${ }^{(107,108)}$. Their adsorptive ability would increase with increased methylation of the hydroxyl moieties on the phenyl-propane units ${ }^{(107,108)}$. Lignins also reduce DNA lesions in rat testicular cells and lymphocytes both in vitro and ex vivo ${ }^{(109)}$. Se inhibits the occurrence of neoplasia in rats and mice, suggesting that an Se-poor diet is associated with an increased prevalence of neoplasia in specific human populations ${ }^{(110)}$. This probably depends on the activity of the selenoprotein glutathione peroxidase, which is involved in the development of cancers $^{(11)}$. Cereal bioactive compounds act via several other anti-mutagenic and anti-carcinogenic mechanisms ${ }^{(112)}$. Important ones are the adsorption and dilution of carcinogens by insoluble dietary fibre and lignins ${ }^{(69,106,113,114)}$, and the action of SCFA produced by fibre fermentation ${ }^{(115)}$. Butyrate is a major factor, as more is produced in the presence of RS, and favours apoptosis in 
human cancer cell lines ${ }^{(62)}$ and DNA repair in rats ${ }^{(116)}$. Interestingly, contrary to what was believed since the works of Burkitt emphasising the preponderant role of fibre in the prevention of Western diseases, notably colon cancer observed in Western countries and not in African rural population consuming high levels of dietary fibre ${ }^{(117)}$, it is more and more believed today that the effect against colon cancer development might be before all attributed to $\mathrm{RS}^{(118)}$, since a lower risk of colon cancer was recently observed in populations with a low level of fibre consumption but with a high intake of $\operatorname{RS}^{(119,120)}$. This reinforces the idea that specific products of RS fermentation within the colon, such as butyric acid, are the active components. Betaine ${ }^{(121)}$ may be added to the list of anti-carcinogenic compounds, as its concentration can reach $0.3 \%$ in whole-grain wheat and $1.5 \%$ in wheat bran (Table 2).

To summarise, the anti-carcinogenic effects of insoluble fibre (including lignin), phytochemicals and wheat bran oil can be distinguished. Insoluble fibre may act directly by adsorbing or diluting carcinogens (through increased faecal bulk by water absorption), or indirectly by decreasing colon $\mathrm{pH}$ (through SCFA production) and increasing butyrate production. The role of phytochemicals is complex and multi-factorial, and notably involves their antioxidant properties since increased oxidative stress is a major factor in the aetiology of cancers ${ }^{(91,122)}$. The exact components of wheat bran oil that reduce the development of colon tumours are still to be identified ${ }^{(89,90)}$. However, animal experiments indicate that dietary fibre, particularly soluble fibre, may not protect against or even enhance carcinogenesis. This may be due to the abrasive property of insoluble fibre, a too low $\mathrm{pH}$ $(<6.5)$ reached within the colon following soluble fibre and RS fermentation, the enhanced colon glucuronidase activity (that converts conjugated carcinogens to free carcinogens) and the increased production of secondary bile acids (tumour promoters) within the colon due to the increased viscosity of some soluble fibre which reduces the reabsorption of bile salt in the small intestine ${ }^{(123)}$.

\section{Whole-grain cereals as a rich source of antioxidants}

Whole-grain cereals can protect the body against the increased oxidative stress that is involved and/or associated with all the major chronic diseases: metabolic syndrome $^{(124)}$, obesity ${ }^{(125,126)}$, diabetes ${ }^{(127,128)}$, $^{(129)^{2}}$ rs $^{(91)}$ and $\mathrm{CVD}^{(129,130)}$. Whole-grain cereals are good sources of antioxidants (thirty-one compounds or groups of compounds are listed in Table 4), as shown by measurements made in vitro of the antioxidant capacity of whole-grain, bran and germ fractions ${ }^{(131-135)}$. However, this may not be the same in vivo ${ }^{(136)}$, and up to today, to my knowledge, the number of studies exploring the in vivo antioxidant effect of wholegrain cereals and/or their fractions in human subjects does not exceed eleven ${ }^{(137-147)}$. The antioxidants in cereals differ in their structure and mode of action ${ }^{(46,136)}$. There are indirect antioxidants, such as $\mathrm{Fe}, \mathrm{Zn}, \mathrm{Cu}$ and $\mathrm{Se}$, which act as cofactors of antioxidant enzymes, and direct radical scavengers such as ferulic acid, other polyphenols (lignans, anthocyanins and alkylresorcinols), carotenoids, vitamin E and compounds specific to cereals other than wheat, such as $\gamma$-oryzanol in rice and avenanthramides in oats. These can neutralise free radicals and/or stop the chain reactions that lead to the production of oxidative radical compounds (for example, the lipid chain peroxidation stopped by vitamin $\mathrm{E}$ within cell membranes). Another antioxidant mechanism involves phytic acid, which can chelate $\mathrm{Fe}$ and thus stop the Fenton reaction producing the highly oxidative and damaging free radical $\mathrm{OH}^{-}$, ultimately reducing lipid peroxidation ${ }^{(148)}$. Lignins are also considered to be antioxidants in vitro (radical-scavenging activity) ${ }^{(149)}$, but precisely how they act in vivo is not known: they may adsorb oxidative damaging compounds within the digestive tract in a way similar to bile salts adsorption ${ }^{(107,108)}$. While the action of cereal antioxidants is not well characterised once the epithelial barrier has been crossed, there is a growing belief that cereal antioxidants protect the intestinal epithelium cells from oxygen-derived free radicals ${ }^{(136,150)}$, particularly those produced by bacteria that may help form active carcinogens by oxidising procarcinogens or those that may result from increased stool Fe content (Fenton reaction) due to a diet high in red meat ${ }^{(151)}$. The concept of 'dietary fibre-bound phytochemicals/phenolic compounds' was proposed recently ${ }^{(18,150)}$. The authors suggest that the antioxidant polyphenols survive digestion in the small intestine because most of them are bound to fibre (for example, esterification of phenolic acids to arabinoxylans) in the cereal food matrix. They reach the colon where the fibre is fermented and some of the antioxidants are released ${ }^{(150)}$. Vitaglione et al. hypothesised 'the slow and continuous release in the gut of the dietary fibre bound antioxidants', such as that of ferulic acid, which will determine the effects of these antioxidants, and considered dietary fibre to be a 'natural functional ingredient to deliver phenolic compounds into the gut ${ }^{(150)}$. For example, only $0.5-5 \%$ of the ferulic acid is absorbed within the small intestine, mainly the soluble free fraction ${ }^{(152-154)}$, and this typical whole-grain wheat phenolic acid (about $90 \%$ of total phenolic acids) would probably exert a major action in the protection of the colon from cancer. Thus, bound antioxidant phenolic acids might act along the whole length of the digestive tract by trapping oxidative compounds. This fraction of bound polyphenols has often led to an important underestimation of the real antioxidant capacity of whole-grain cereals - and of their fractions - as measured in vitro and generally based on the measurement of the easily extractable polyphenol fraction $^{(133,155)}$. In vivo studies are now needed to examine this hypothesis, and to characterise and quantify this potential antioxidant effect within the digestive tract.

The antioxidants in whole-grain cereals act via different, complex, and synergetic mechanisms in vivo. However, the antioxidant action of whole-grain cereals has not yet been convincingly validated in human subjects and requires further exploration.

\section{Whole-grain cereals as rich sources of magnesium}

Among plant-based foods, whole-grain cereals, together with legumes, nuts and seeds, are one of the best sources of $\mathrm{Mg}$ : whole-grain wheat contains $104 \mathrm{mg} \mathrm{Mg} / 100 \mathrm{~g}$, wheat bran $515 \mathrm{mg}$, and wheat germ $245 \mathrm{mg}$ (Table 2). The high $\mathrm{Mg}$ content of whole-grain cereals may explain its favourable impact on insulin sensitivity and diabetes risk 
(Fig. 2) ${ }^{(156)}$, diabetes being otherwise frequently associated with $\mathrm{Mg}$ deficiency ${ }^{(157)}$. Mg can increase insulin secretion and the rate of glucose clearance from the blood in humans ${ }^{(158,159)}$. This was also proposed to explain the lower insulin response in obese and overweight adults following the consumption of a whole-grain-based diet as compared with those on a refined cereal-based diet ${ }^{(160)}$. High-Mg diets reduce insulin resistance in rats fed a high-fructose diet ${ }^{(161)}$. they also reduce the development of spontaneous diabetes in obese Zucker rats, a model of non-insulin-dependent diabetes mellitus, but these rats had to be given $\mathrm{Mg}$ before the onset of diabetes to obtain protection ${ }^{(162)}$. Most explanations of the prevention of type 2 diabetes by $\mathrm{Mg}$ are based on the finding that $\mathrm{Mg}$ stimulates insulindependent glucose uptake in elderly subjects ${ }^{(158,163)}$. It also protects Mg-deficient animals from the production of reactive oxygen species ${ }^{(164)}$. Reactive oxygen species are partly responsible for the increased hyperglycaemiamediated oxidative stress in diabetic subjects ${ }^{(165,166)} . \mathrm{Mg}$ also acts as a mild physiological $\mathrm{Ca}$ antagonist ${ }^{(167)}$. Obese and diabetic patients with insulin resistance have excess free intracellular $\mathrm{Ca}$ and these two clinical conditions are associated with hypertension ${ }^{(168)}$. In addition, $\mathrm{Mg}$ helps keep the concentration of intracellular $\mathrm{Ca}$ optimal through various complex cellular mechanisms involving $\mathrm{Ca}$ channels, $\mathrm{Ca}$ sequestration/extrusion by the endoplasmic reticulum and $\mathrm{Ca}$ binding sites on proteins and membranes ${ }^{(156)}$. Finally, low serum plasma $\mathrm{Mg}$ has been positively associated with a higher risk of coronary atherosclerosis or acute thrombosis ${ }^{(169)}$, suggesting that whole-grain cereal $\mathrm{Mg}$ might also contribute to the prevention of CVD. This may also involve the inhibition of platelet-dependent thrombosis by $\mathrm{Mg}$ supplementation in patients with coronary artery disease ${ }^{(170)}$ and the positive effect of $\mathrm{Mg}$ upon blood pressure regulation in hypertensive patients $^{(171)}$. The capacity of a regular prolonged consumption of whole-grain cereals to sustain a high plasma $\mathrm{Mg}$ concentration therefore deserves to be investigated in the context of type 2 diabetes prevention.

\section{The action of some anti-nutrients on starch hydrolysis and glycaemia}

Whole-grain cereals are also a source of antinutrients with both adverse and positive health effects. The most important are phytic acid, lectins, tannins, saponins and inhibitors of enzymes such as proteases and $\alpha$-amylases. Their main negative effect is their ability to reduce the bioavailability and the absorption of some nutrients (for example, the chelation of minerals by phytic acid and tannins), the binding of lectins to epithelial cells that damages the intestinal microvillae, and inhibition of digestive enzymes by tannins, which inhibits growth in animals ${ }^{(172,173)}$. Cereal products in the human diet are cooked; this leads to losses of antinutrients such as lectins and enzyme inhibitors, and the major health outcome appears to be the low dietary Fe bioavailability in African populations that consume sorghum or finger millet-based beverages, gruels and porridges, both cereals containing phytic acid and a high tannin content ${ }^{(174,175)}$. For example, the phytate and Fe-binding phenolic compounds in whole-grain millet flour may reach $0.6 \mathrm{~g} / 100 \mathrm{~g}(\mathrm{DW})^{(176)}$. This is one of the key factors responsible for Fe-deficiency anaemia in developing countries ${ }^{(175)}$. On the other hand, the use of traditional processing such as germination, soaking, pre-fermentation and cooking may help to decrease the tannin and phytic acid contents, so improving $\mathrm{Fe}$ bioavailability $(177-180)$.

However, phytic acid, lectins, protease inhibitors and tannins also contribute to the low-GI property of wholegrain foods ${ }^{(181,182)}$. In wheat and derived whole-grain food

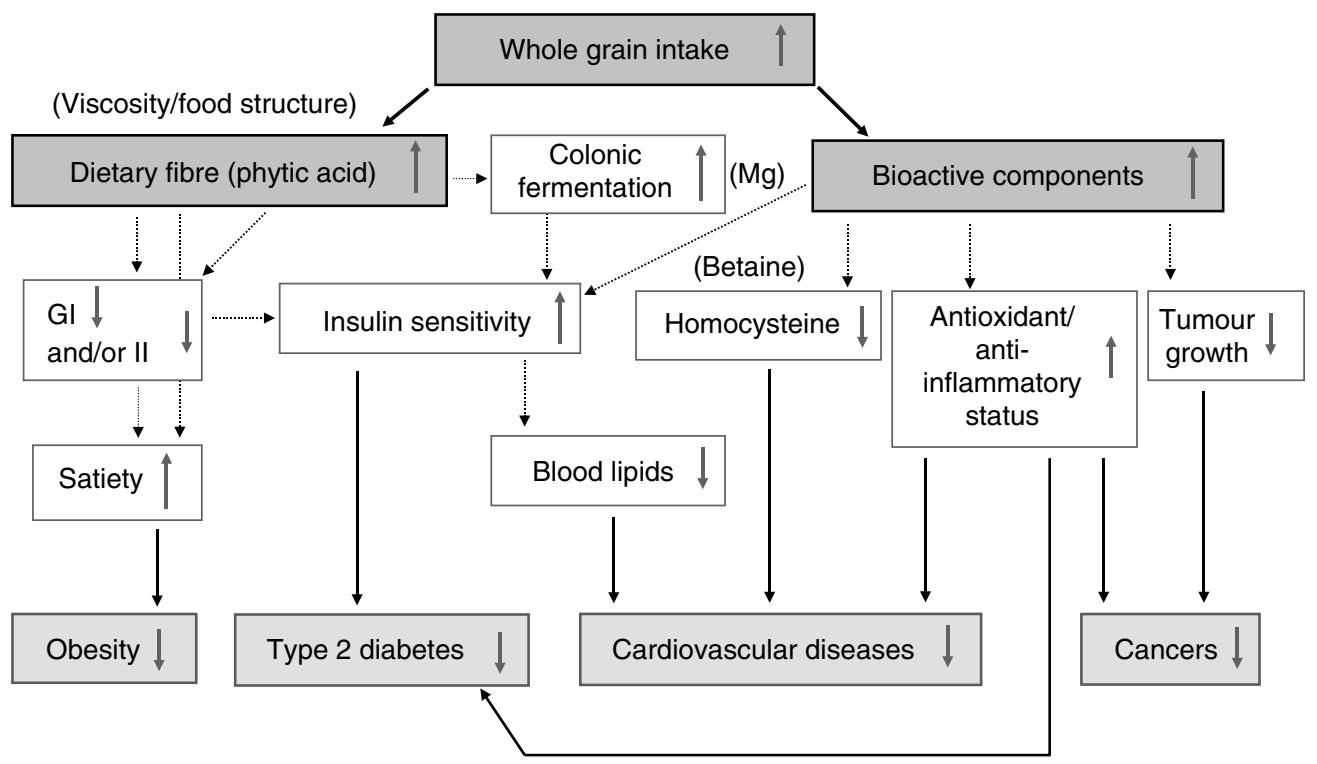

Fig. 2. Current accepted mechanisms for how whole grain protects against major chronic diseases (modified with permission from Professor I. Björck (University of Lund, Sweden); see the HealthGrain brochure for original diagram: 'Progress in HEALTHGRAIN 2008', a project from the European Community's Sixth Framework Programme, FOOD-CT-2005-514008, 2005-2010; see Poutanen et al. ${ }^{(478)}$ for more details about the Project). Gl, glycaemic index; II, insulinaemic index. 
products, since lectins and enzyme inhibitors are inactivated by cooking processes, this is primarily phytic acid which would reduce glycaemia through several potential mechanisms: thus, binding with proteins closely associated with starch, association with digestive enzymes, chelation of $\mathrm{Ca}$ required for $\alpha$-amylase activity, direct binding with starch, effect on starch gelatinisation during cooking processes and slowing of gastric emptying rate might be involved ${ }^{(181)}$.

\section{Conclusion}

The proposed mechanisms by which whole-grain cereals may protect the body are shown in Fig. 2. The most important ones are the preservation of food structure, fibre fermentation in the colon, the hypoglycaemic and hypoinsulinaemic, antioxidant, anti-inflammatory and anticarcinogenic properties of several bioactive compounds, improved insulin sensitivity by $\mathrm{Mg}$ and reduced hyperhomocysteinaemia by betaine, a significant CVD risk factor (for details about betaine, see the 'New hypotheses' section below). However, an extensive list of all the bioactive compounds in whole-grain wheat and its fractions (Table 2), the ways they act and their health effects as isolated free compounds (Tables 3 and 4) makes it possible to formulate new hypotheses to explain the protective role of whole-grain cereals. Whole-grain cereals, particularly wheat and/or wheat bran and germ, are also a source of $n-3$ fatty acids (especially $\alpha$-linolenic acid), sulfur compounds (reduced glutathione (GSH), oxidised glutathione (GSSG), methionine and cystine), oligosaccharides (fructans, raffinose and stachyose), $\mathrm{P}, \mathrm{Ca}, \mathrm{Na}, \mathrm{K}, \mathrm{B}$ vitamins, flavonoids (for example, anthocyanins and isoflavonoids), alkylresorcinols, betaine, choline, phytosterols, inositols, policosanol and melatonin. The actions of these compounds will be described in the next 'New hypotheses' section. The antioxidant hypothesis will be discussed with a broader perspective, as well as the health benefits of active compounds from whole-grain cereals that are less often studied, such as B vitamins, sulfur compounds, methyl donors and lipotropes, $\alpha$-linolenic acid, lignins, oligosaccharides, policosanol and melatonin.

\section{New hypotheses: a broader perspective for the protective action of whole-grain cereals}

The antioxidant hypothesis must not be reduced to free radical scavenging and antioxidant enzyme activation

There is more and more evidence that the primary effect of antioxidants from whole-grain cereals is in the digestive tract, where they protect intestinal epithelial cells from attack by free radicals ${ }^{(136,150)}$. However, the mechanisms by which antioxidants that cross the intestinal barrier protect the body remain uncertain. Published studies on animals and human subjects fed the free compounds give rise to new explanations of the antioxidant protection by whole-grain cereals. The antioxidant action of whole-grain cereals might be multi-factorial and much more complex than it first appears. There are at least four new mechanisms to be studied in the context of whole-grain cereals: the action of polyphenols on cell signalling and gene regulation modifying the redox status of tissues and cells, the action of sulfur amino acids on glutathione synthesis, the possible stimulation of endogenous antioxidants by whole-grain cereal bioactive compounds, and the underestimated antioxidant properties of phytic acid and lignin.

Whole-grain cereals as a source of polyphenols involved in cell signalling. The polyphenols in complex foods are generally not readily absorbed in the small intestine: $2-5 \%$ for whole-grain cereal phenolic acids (Table 2), and 30$40 \%$ for flavonoids from vegetables, beverages and fruits, depending on the food ${ }^{(183)}$. The resulting plasma concentrations of these absorbed compounds are generally in the nanomolar (nM) or micromolar $(\mu \mathrm{M})$ range, lower than that of endogenous antioxidant compounds such as GSH and vitamin $\mathrm{C}$ (millimolar). However, this does not mean that they have no antioxidant action. Some quite recent studies on isolated compounds have shown that flavonoids ${ }^{(184,185)}$ and phenolic acids ${ }^{(186,187)}$ act on cell signalling pathways, so modifying gene regulation and/or cell redox status, as has been discussed in several recent reviews ${ }^{(188-191)}$. However, most of the studies were performed with flavonoids, not phenolic acids which are more abundant in whole-grain wheat (up to $100 \mathrm{mg} / 100 \mathrm{~g}$ ) than are flavonoids (30$43 \mathrm{mg} / 100 \mathrm{~g}$ ) (Table 2). Results obtained with isolated flavonoids, mainly in in vitro cell cultures, may be extrapolated to flavonoids found in whole-grain wheat once they have entered the bloodstream and then reached cells. Little work has been done to precisely identify wheat flavonoids. Nevertheless, some of them are catechin and proanthocyanidins $^{(192)}$, tricine $e^{(69)}$, apigenin glycosides ${ }^{(193)}$, and vicenin and schaftosides ${ }^{(194)}$. These flavonoids may act as signals within cells. The main mechanisms probably involve the redox status and antioxidant and pro-inflammatory genes activated by increased oxidative stress, i.e. a modified redox state of the cell, through signalling pathways that may be up- and down-regulated by polyphenols via activation or inactivation of transcription factors such as NF- $\kappa B^{(189,187)}$ or activator protein-1 (AP-1) ${ }^{(186)}$. Thus, flavonoids can increase GSH synthesis through the transcription factor Nrf2 (nuclear factor-erythroid 2-related factor 2) which binds to specific antioxidant/electrophile response element (AREs/EpRE)-containing gene promoters $^{(188)}$. For example, oxidised quercetin (quinone) can react with thiols in the Keap1 protein (Kelch-like ECH-associated protein 1 bound to the cytoskeleton), releasing Nrf2 and then activating specific genes via ARE/EpRE involved in GSH synthesis $^{(188)}$. Here, more than the antioxidant property of the flavonoids, it is its activated or metabolised form which would be active within cells. Kaempferol and quercetin, two flavonoids, also modulate the production of $\gamma$-glutamylcysteine synthetase ${ }^{(195)}$, an important enzyme in the synthesis of GSH. The authors conclude that flavonoids are important for regulating the intracellular concentration of $\mathrm{GSH}^{(195)}$. There is therefore a strong link between the intra- and/or extracellular actions of polyphenols, redox cell status and gene regulation, broadening the notion of antioxidant polyphenols to activities other than just free radical scavenging. However, most studies have used higher polyphenol concentrations $(>10 \mu \mathrm{M})$ than those found in vivo. For example, the postprandial plasma ferulic acid concentrations following 
wheat bran consumption in rats were about $1 \mu \mathrm{M}^{(154)}$ and about $0 \cdot 2 \mu \mathrm{M}$ in human subjects ${ }^{(196)}$. However, a study conducted in vitro on cell cultures with six wine phenolic acids in the $20 \mathrm{nM}-20 \mu \mathrm{M}$ range showed that ferulic, sinapic, $p$-coumaric and caffeic acids (all found in whole-grain wheat) are able to inhibit the action of pro-inflammatory transcription factor AP-1 as low as $20 \mathrm{~nm}$ in a range of $5-15 \%{ }^{(186)}$. Besides, it may reasonably be supposed that the true plasma polyphenol concentration is higher than the $0 \cdot 2-1 \mu \mathrm{M}$ reached with ferulic acid due to the presence of other polyphenols such as sinapic acid and, to a lesser extent flavonoids, as recently reported in human subjects where $\mathrm{a}+5 \mu \mathrm{M}$ increase in plasma total polyphenols has been observed $1 \mathrm{~h}$ after boiled wheat bran consumption ${ }^{(146)}$. Most of the sinapic acid in whole-grain wheat is free or in a soluble conjugated form (approximately equal to $70 \%$ ), and may reach a total concentration of $4-18 \mathrm{mg} / 100 \mathrm{~g}$ whole-grain wheat ${ }^{(197)}$. However, whether the low plasma polyphenol concentrations obtained following a whole-grain cereal meal are compatible with cell signalling activity remains to be explored.

Whole-grain cereals are a rich source of sulfur compounds. The sulfur amino acid contents (methionine and cystine) of whole-grain wheat, wheat bran and germ are 0.5 , 0.6 and $1.0 \%$ (Table 2), and may be higher in some cereal varieties (see ranges in Table 2). Methionine and cystine are both precursors of GSH, an intracellular antioxidant, and as such contribute to the control of the cell oxidative status by participating in gene expression through modification of the thiol redox status, as has been recently reviewed ${ }^{(198,199)}$. Thus, rats fed a $0.6 \%$ free methionine diet had a higher hepatic GSH content than rats fed a control $10 \%$ caseinbased diet without methionine supplementation ${ }^{(200)}$. It has also been shown in rat gut mucosa and plasma that an inadequate intake of sulfur amino acids leads to the oxidation of the thiol/disulfide redox status (expressed by the ratios cysteine:cystine and GSH:GSSG), i.e. a less reductive potential, that in the end increases oxidative stress $^{(201)}$. Methionine also generates cysteine via the cystathionine pathway ${ }^{(202)}$, cysteine being oxidised to cystine (two cysteine moieties linked by a disulfide bond).

For humans, average daily intakes of $305-2770 \mathrm{mg}$ methionine and 197-1561 mg cystine have been reported for a usual diet ${ }^{(203)}$. The estimated daily requirements of methionine + cysteine are $910-2100 \mathrm{mg} / \mathrm{d}$ for a $70 \mathrm{~kg}$ adult ${ }^{(204)}$. Based on the methionine and cystine content of commercially prepared whole-wheat bread (USDA database, 155 and $214 \mathrm{mg} / 100 \mathrm{~g})^{(205)}$ and on a daily consumption of one serving of whole-grain cereal products (i.e. about $30 \mathrm{~g}$ for a slice of bread) ${ }^{(206)}$, whole-grain cereals provide an average $47 \mathrm{mg}$ methionine and $64 \mathrm{mg}$ cystine per d. This suggests that whole-grain cereals contribute little to methionine and cystine intakes, at least for low consumers. However, quite significant amounts of at least $280 \mathrm{mg}$ methionine and $380 \mathrm{mg}$ cystine per d can be obtained by following the USDA food guide pyramid that recommends between six and eleven daily servings of whole-grain cereal products. This would significantly contribute either to the average daily intakes as previously reported ${ }^{(203)}$ or to the daily recommendations ${ }^{(204)}$. However, it is not known how a regular daily consumption of between six and eleven servings of whole-grain cereal products would contribute to GSH synthesis and/or an improved antioxidant status in humans.

GSH can be hydrolysed in the small intestine by $\gamma$ glutamyltransferase and/or absorbed intact, mainly in the upper jejunum ${ }^{(207)}$. It is therefore available to cells where it may exert its physiological effects as an antioxidant, anticarcinogenic and/or immunostimulating ${ }^{(208)}$ agent and also as detoxifier of xenobiotics. Human subjects given a solution of $46 \mathrm{mg} \mathrm{GSH} / \mathrm{kg}$ body weight (a single oral dose of $3 \mathrm{~g}$ ) showed no significant increase in postprandial plasma $\mathrm{GSH}^{(209)}$. Dietary GSH, but also its dietary precursors methionine and cystine, are therefore not major determinants of circulating $\mathrm{GSH}^{(203)}$, probably because GSH is rapidly hydrolysed in the small intestine ${ }^{(209)}$; however, it might help detoxify reactive electrophiles in the diet within the intestinal lumen ${ }^{(207)}$ or protect epithelial cells against attack by free radicals. The human daily total GSH consumption is $13-110 \mathrm{mg}$ (mean $35 \mathrm{mg})^{(203)}$. Using the GSH highest content in whole-grain wheat (Table 2), that is about $5.7 \mathrm{mg} /$ $100 \mathrm{~g}$, and eating $30 \mathrm{~g}$ whole-grain cereal per $\mathrm{d}$ as bread (about $38 \%$ water), it may be calculated that whole-grain bread provides less than $1.3 \mathrm{mg}$ GSH per $\mathrm{d}$. Increasing the consumption of whole-grain cereal products to between six and eleven servings daily as recommended by the USDA food pyramid (epidemiological data show that an average 2.7 servings of whole-grain foods have beneficial health effects), especially servings containing wheat germ since this fraction may have $246 \mathrm{mg} \mathrm{GSH} / 100 \mathrm{~g}$ - and probably more if total glutathione equivalents $(\mathrm{GSH}+(2 \times \mathrm{GSSG})+$ proteinbound glutathione) are considered - might therefore provide a substantial supply of GSH. Thus, the total GSH content of high-grade extraction wheat flours $(1.44-1.73 \mathrm{~g}$ ash/100 $\mathrm{g}$ ) is $11.6-17.6 \mathrm{mg} / 100 \mathrm{~g}$ (with a water content for whole-grain wheat flour of $13.0 \%$ ), which is about three times the total GSH content of low-grade extraction wheat flours $(0.54-0.59 \mathrm{~g}$ ash $/ 100 \mathrm{~g}$ and $4.7-5.0 \mathrm{mg}$ total $\mathrm{GSH} / 100 \mathrm{~g}$ flour with an $11.9 \%$ water content for white wheat flour), clearly showing that GSH is mainly in the bran ${ }^{(210)}$. However, a higher total glutathione content of $15.8 \mathrm{mg} / 100 \mathrm{~g}$ (thirty-six wheat varieties) was evaluated from data by $\mathrm{Li}$ et al. for white wheat flours ${ }^{(211,212)}$. The contribution of total whole-grain wheat GSH to the antioxidant defence, either within the gut lumen or as a substrate supplying cysteine for endogenous GSH synthesis in the liver, might be explored by comparing low-methionine and whole-grain-rich diets.

The possible action of whole-grain cereal compounds on plasma uric acid level. A recent study on human subjects consuming apples demonstrated that the elevated plasma postprandial antioxidant level $(+55 \mu \mathrm{M}$ trolox equivalents after $1 \mathrm{~h}$ and stabilisation at about $+20 \mu \mathrm{M}$ trolox equivalents between 2 and $6 \mathrm{~h}$; ferric-reducing ability of plasma (FRAP) assay) was due to increased uric acid and not to a significant increase in plasma vitamin $\mathrm{C}$ or polyphenols ${ }^{(213)}$. Fructose was thought to stimulate adenine nucleotide degradation leading to uric acid synthesis ${ }^{(214)}$. The authors proposed that the increased plasma antioxidant level following consumption of flavonoid-rich diets is due to an increase in uric acid, while sucrose, sorbitol, lactate 
and/or methylxanthines are also candidates for endogenous uric acid synthesis ${ }^{(214)}$. Uric acid is a powerful antioxidant whose concentration in human plasma can reach 160 $450 \mu \mathrm{M}$, and can account for as much as $40-90 \%$ of the plasma antioxidant capacity ${ }^{(214)}$. A recent study on human subjects has shown that there is little or no correlation between changes in plasma total phenolic acids and antioxidant capacity (FRAP assay) following the consumption of wheat bran, indicating that compounds other than phenolic acids contribute to the postprandial increase in plasma antioxidants to about $+50 \mu \mathrm{m}$ of FRAP between 1 and $3 \mathrm{~h}^{(146)}$. This increase is in the same range as that found by Lotito \& Frei with apples ${ }^{(213)}$ and with other values reported by Price et al. with tea, red wine, spinach and strawberries, from +15 to $+100 \mu \mathrm{M}$ increase in plasma FRAP $^{(146)}$. This cannot be explained by the low fructose content of wheat bran (about $50 \mathrm{mg} / 100 \mathrm{~g}$ ), much lower than that of apples (about $5 \cdot 7 \mathrm{~g} / 100 \mathrm{~g}$ ) ${ }^{(215)}$. However, whole-grain cereals contain an important package of bioactive compounds other than fructose or polyphenols whose effect upon endogenous antioxidant synthesis has not been explored. It would be therefore relevant to confirm this increase in plasma antioxidant level following wheat bran consumption, and to identify the mechanisms underlying such an increase, which is apparently not due to the increase in circulating plasma polyphenols alone ${ }^{(146)}$. Work is also needed to determine whether the consumption of wholegrain cereals and/or bran and germ fractions can significantly increase the plasma uric acid concentration to those produced by coffee $(+5 \%)$ or tea $(+7 \%)^{(216)}$.

Whole-grain cereals as a source of phytic acid and lignins. Phytic acid from whole-grain cereals has long been considered to be nutritionally negative, since it chelates minerals such as $\mathrm{Zn}, \mathrm{Fe}, \mathrm{Ca}$ and/or $\mathrm{Mg}$, thus limiting their intestinal bioavailability ${ }^{(217)}$. This has been used as an argument for using refined flours instead of wholemeal wheat flours. However, phytic acid is also a strong antioxidant in vitro ${ }^{(218)}$, and may reach $6 \%$ in the bran of certain wheat varieties (Table 2). It therefore needs to be determined whether the negative effect of phytic acid on mineral assimilation can be offset by its antioxidant activity and the high content in minerals of whole-grain wheat. Today, the answer to this is undoubtedly 'yes'. First, the quantity of mineral chelated by phytic acid is apparently not high enough compared with the much greater quantity in whole-grain cereals compared with refined ones. Rats fed whole-wheat flour absorbed more minerals than rats fed white wheat flour ${ }^{(219)}$. Besides, baking bread according to a sourdough procedure can activate endogenous phytases and lower the $\mathrm{pH}$, thus limiting the chelation of minerals by phytic acid ${ }^{(22)}$. Second, it is now known that phytic acid can chelate $\mathrm{Fe}$, thus limiting the damage due to the Fenton reaction leading to the production of the very reactive free radical $\mathrm{OH}$. Third, the phytate in whole grain is accompanied by other bioactive compounds that are lost during refining. Phytic acid is therefore a serious candidate as a whole-grain cereal antioxidant acting in vivo. Unfortunately, I know of no studies that have explored the antioxidant effect of this compound from whole-grain cereals in vivo.
The concentration of lignins in whole-grain wheat is $1.9 \%: 5.6 \%$ in wheat bran and $1.5 \%$ in germ (Table 1). Lignins are absent from refined flour and are generally considered to be nutritionally inert. However, some studies have demonstrated its potential positive physiological effects. Studies on rats showed that lignin may account for $26-32 \%$ of the enterolactone (a mammalian lignan) formed from cereal bran $^{(221)}$. Mammalian lignans are antioxidants in vitro at the concentrations $(10-100 \mu \mathrm{M})$ achievable in vivo ${ }^{(222)}$, particularly in the colon ${ }^{(223)}$. A study on rats fed a diet containing $8 \%$ lignin for $21 \mathrm{~d}$ showed that lignins can have antioxidant effects on ex vivo fresh lymphocytes by significantly decreasing the peroxide-induced DNA strand breaks and visible light-induced oxidative DNA lesions under the form of oxidised bases via singlet oxygen $-{ }^{1} \mathrm{O}_{2}-$ production ${ }^{(224)}$. But I know of no studies on human subjects that have examined the physiological effects of lignins. However, if lignins are partially metabolised to mammalian lignans in humans, as they are in rats, they might add to the protection by lignans observed in human subjects against some cancers ${ }^{(96)}$. Again, studies are needed to explore the antioxidant effect of whole-grain cereal lignins in vivo.

\section{Whole-grain cereals as a source of bioactive compounds with underestimated physiological effects}

Whole-grain cereals as a source of lipotropes and methyl donors: betaine, choline, folates, methionine and myoinositol. Betaine and choline are now recognised as important in human nutrition: betaine improves the health of the heart, liver and kidneys, while choline is important for lipid metabolism, brain development, the integrity and signalling function of cell membranes, and as a precursor of phosphatidylcholine, acetylcholine and betaine $(\text { Table } 3)^{(225,226)}$. The nutritional role of folates (vitamin $\mathrm{B}_{9}$ ) is also well recognised, particularly in the prevention of neural tube defects and CVD (Table 3). What is more surprising is that their contribution to the health benefits of whole-grain cereals, particularly wheat bran and wheat germ, has not been recognised until very recently (Fig. 2) ${ }^{(136,227)}$. Whole-grain wheat, wheat bran and wheat germ, respectively, contain about $0.28,1.04$ and $1.09 \%$ betaine and choline and about 51, 231 and $420 \mu \mathrm{g}$ folates $/ 100 \mathrm{~g}$ (Tables 1 and 2). However, whole-grain cereals are not very good sources of folates as compared with legumes or vegetables, notably when based on a $100 \mathrm{kcal}$ $(420 \mathrm{~kJ})$ content $^{(228)}$. The bioavailability of choline and betaine from whole-grain cereal products and fractions is not known. However, its presence as a free soluble osmolyte (225) $^{(25)}$ in cells of the aleurone layer suggests that betaine is readily available, especially compared with fibre-bound antioxidant polyphenols. To my knowledge, only two studies, using the metabonomic approach, have underlined the importance of betaine from whole-grain cereals by showing an increased hepatic, urinary and plasma betaine levels in rats and pigs fed whole-grain wheat flour and high-fibre rye bread 229,230$)$. This suggests that betaine from whole-grain cereals is quite available. It has also been recently shown that free betaine can reverse insulin resistance and liver injury in mice fed a high-fat diet, an animal model of non-alcoholic fatty liver disease $^{(231)}$. Thus, the probably high bioavailability of 
betaine from cereals ${ }^{(229,230)}$ combined with its many described health effects ${ }^{(22)}$ suggest that whole-grain cereal betaine may have multivariate health benefits.

Betaine, choline and folates are all methyl donors, able per se to transform homocysteine into methionine, thereby decreasing hyperhomocysteinaemia ${ }^{(232)}$, a known risk factor for CVD ${ }^{(233)}$, and also for neural tube defects ${ }^{(234)}$ and cancers ${ }^{(235)}$. The dietary intake of whole-grain and bran, but not germ, is significantly and negatively associated with the plasma homocysteine concentration: -17.4 and $-10.9 \%$ when comparing the highest and lowest quintiles of whole-grain and bran cereal intake, respectively ${ }^{(17)}$. The wide variety of micronutrients may interact in synergy in this effect ${ }^{(17)}$. More precisely, one may hypothesise that folates, betaine and choline would be primarily involved. Besides, since hyperhomocysteinaemia is associated with increased oxidative stress ${ }^{(236,237)}$, betaine and choline may act as indirect antioxidants.

Betaine, choline and folates are also lipotropic compounds, together with methionine and myo-inositol, that are essential for lipid metabolism, DNA methylation and the production of nucleoproteins and membranes ${ }^{(225,226,238-240)}$. By definition, a lipotrope is a substance that specifically prevents excess fat deposition in the liver by hastening fat removal or by limiting lipid synthesis. However, using this definition sensu strictu, very few studies on human subjects have been published; most have been performed on animals. It is estimated that wholegrain wheat, wheat bran and wheat germ can supply $0 \cdot 51$, 1.31 and $1.59 \mathrm{~g}$ lipotropes $/ 100 \mathrm{~g}$, respectively (Table 2). These values could be higher if other compounds with indirect lipotrope-like effects are included (those that indirectly prevent fat accumulation) such as $\mathrm{Mg}$, niacin, pantothenic acid, RS, some flavonoids, PUFA, phytic acid, lignans, some oligosaccharides and fibre. Among lipotropes, as for choline, myo-inositol (a carbocyclic polyol) is derived from several myo-inositol-derived compounds that are essentially free myo-inositol and conjugated myo-inositol, either with glycosylated (for example, galactinol and di-galactosyl myo-inositol) or phosphorylated (for example, phytate or hexakisphosphate) groups. However, the lipotropic effect of phytate has not yet been demonstrated in human subjects and is probably low since human phytases are much less active than those in the rat small intestine ${ }^{(241)}$. In addition, among the nine isomers of inositol, only myo-inositol has been shown to be lipotropic, not chiro-inositol $^{(242)}$, which is abundant in the pseudo-cereal buckwheat $^{(243,244)}$ and is mainly known for its action against insulin resistance and its ability to help controlling blood glucose $^{(245)}$. Except for myo-inositol phosphate (from hexakisphosphate to monophosphate) contents, there are few data on the free myo-inositol content of whole-grain cereals and their bran and germ fractions before processing. To my knowledge, the only published values are $86.7 \mathrm{mg} /$ $100 \mathrm{~g}$ for whole-grain amaranth ${ }^{(246)}, 8.5 \mathrm{mg} / 100 \mathrm{~g}$ for oats $^{(247)}, 30.8-35.4 \mathrm{mg} / 100 \mathrm{~g}$ for whole-grain quinoa ${ }^{(248)}$ and $52.5 \mathrm{mg} / 100 \mathrm{~g}$ for dry mature wheat embryo ${ }^{(249)}$, which is quite similar to the germ fraction. The same authors also reported that dry mature wheat embryo contained about $56 \mathrm{mg}$ galactinol/100 $\mathrm{g}^{(249)}$. Myo-inositol is therefore mainly present in phytate in cereal grains, about $95 \%$ in wheat ${ }^{(250)}$.
I have used this percentage and the phytic acid content of whole-grain wheat to estimate the free myo-inositol contents of whole-grain wheat, wheat bran and wheat germ (Table 2). The total myo-inositol content of 487 foods was published in 1980, forty-seven of which were processed cereal-based products (twenty-four types of bread, fifteen breakfast cereals and eight kinds of pasta). The total myo-inositol/ $100 \mathrm{~g}$ was $25-1150 \mathrm{mg}$ for wheat breads and $7-35 \mathrm{mg} /$ $100 \mathrm{~g}$ for wheat-derived breakfast cereals ${ }^{(251)}$. Considering all cereal foods, the values given were then within the range $6-1150 \mathrm{mg} / 100 \mathrm{~g}$ for breads and $2-274 \mathrm{mg} / 100 \mathrm{~g}$ for other cereal foods (pasta and breakfast cereals) ${ }^{(251)}$. But these values are for total myo-inositol after acid hydrolysis for $40 \mathrm{~h}$ at $120^{\circ} \mathrm{C}$, which releases myo-inositol from phytate in addition to free $m y o$-inositol ${ }^{(251)}$. Nevertheless, hydrolysis of phytic acid within lower inositol phosphate esters (from inositol pentaphosphate to inositol monophosphate and free myo-inositol) by activated endogenous food phytases, through, for example, sourdough baking with natural leaven $^{(220)}$ and/or simple fermentation with yeast ${ }^{(252)}$ and/ or germination ${ }^{(247,252,253)}$, may lead to free myo-inositol formation $^{(247,254)}$, as was shown by using different hydrothermal processes with lactic acid and whole barley kernels $^{(255)}$. Free myo-inositol may then become available for absorption depending on the quantity not degraded by microflora, either during pre-fermentation or in the colon. Thus, the total free myo-inositol content of wheat products is difficult to ascertain precisely and probably depends on the processing parameters (which would explain the high value ranges found for breads). But it is not insignificant. Once ingested, except for folates whose bioavailability would be low when originating from cereal products, other cereal lipotropic compounds are quite readily available in the digestive tract (Table 2), myo-inositol being likely to be further partly converted into chiro-inositol after absorption, as shown in rats ${ }^{(256)}$.

Wheat bran and germ are rich in choline, which is important in lipid metabolism and DNA methylation. Choline, as choline bitartrate, is often used as a lipotrope in animal diets ${ }^{(257)}$, and rats fed a choline-free diet for 14 months develop severe hepatic lesions, hepatic DNA undermethylation and cellular carcinomas ${ }^{(258)}$, DNA undermethylation being related to carcinogenesis development ${ }^{(259)}$, as demonstrated for benign and malignant human colon neoplasms ${ }^{(260)}$. The extent to which lipotropes from whole-grain wheat such as choline help improve lipid status, by preventing fat deposition in the liver, and in balancing DNA methylation in the liver and colon deserve to be explored in prolonged trials with a whole-grain cerealbased diet. In addition to the well-known anti-carcinogenic property of several whole-grain cereal compounds (Table 4), that of choline ${ }^{(260)}$ and betaine ${ }^{(121)}$ should be studied more thoroughly, more particularly at the colorectal level.

The specific actions of bound and free ferulic acid. The physiological action of ferulic acid from whole grain has undoubtedly been underestimated because it is poorly absorbed by the small intestine $(<5 \%$; Table 2$)$, and because most studies have been conducted with the free compound at high and often unrealistic nutritional levels. These studies have nevertheless underlined the potential 
role of ferulic acid as an antioxidant, anti-microbial, antiapoptotic, anti-ageing, anti-inflammatory, neuroprotective, hypotensive, pulmonary-protective and cholesterollowering agent in metabolic diseases such as thrombosis, atherosclerosis, cancer and diabetes (Tables 3 and 4$)^{(104,261,262)}$. However, there have been few studies on the capacity of ferulic acid from cereal products to improve some physiological functions in human subjects ${ }^{(104)}$. Ferulic acid may reach up to $0.2 \%$ of whole-grain wheat and over $0.6 \%$ of wheat bran (Table 2), which is quite significant; and $80 \%$ of ferulic acid is in the bran fraction $^{(263)}$. Since no more than $5 \%$ of ferulic acid is absorbed by the intestine ${ }^{(153)}$, about $95 \%$ reaches the colon bound to fibre where it may act as a natural antioxidant on epithelial cells ${ }^{(150)}$. Thus, both free and metabolised ferulic acid (mainly sulfated and glucuronated) may have a signalling function within cells, and the bound compound might be a strong protective antioxidant and antiinflammatory agent within the colon. The bacterial esterases in the colon will also partially and relatively slowly solubilise bound ferulic acid, as shown in vitro in a human model colon ${ }^{(264)}$. The possible absorption of ferulic acid within the colon and the physiological effects of its metabolites produced by the colon microbiota remain therefore to be quantified and qualified.

The specific actions of lignins. I have discussed the potential role of lignin as an antioxidant. However, lignin is one of the main non-energy-producing compounds in whole grain (about $1.9 \%$ of whole-grain wheat, $5.6 \%$ of wheat bran and $1.5 \%$ of wheat germ) (Table 1). Although generally considered to be nutritionally inert, such a high concentration should have physiological effects, such as protecting the gut epithelium against oxidative damage and protecting other cell wall compounds against fermentation, so increasing faecal bulk and the associated positive health effects (dilution of carcinogens). Some studies support the hypothesis that lignins are not nutritionally inert. For example, bioactive lignophenol derivatives from bamboo lignin are anti-carcinogenic in human neuroblastoma SHSY5Y cells, where they suppress oxidative stress-induced apoptosis $^{(265)}$. It has also been shown that cell walls containing lignins (hydrophobic polymers) favour the adsorption of hydrophobic carcinogens and their release in the faeces ${ }^{(266)}$. Lignins from wheat bran also adsorb bile salts (i.e. bile salt-sequestering agent) such as deoxycholate in vitro, but a link between cholesterol lowering and wheat bran consumption was not demonstrated ${ }^{(267)}$. Lignin may reduce bile salt reabsorption in vivo by adsorbing them ${ }^{(268)}$, and may further reduce the formation of carcinogenic metabolites from bile salts by colon bacteria ${ }^{(269)}$. The lignin nordihydroguairetic acid is also able to prevent changes in renal morphology, by reducing oxidative stress, in rats with diabetic nephropathy for which reactive oxygen species play an important role in its development as a result of chronic hyperglycaemia $^{(270)}$. Finally, lignins from fractionated hardwood hydrolysate, when consumed during 3 weeks from an $8 \%$ lignin-based diet, are able to decrease $\mathrm{H}_{2} \mathrm{O}_{2}$ and visible light-induced DNA damage in ex vivo fresh rat blood lymphocytes ${ }^{(224)}$ and in testicular cells ${ }^{(109)}$. This suggests that lignin compounds or some of their metabolites have crossed the epithelial barrier, or at least have been able to induce antioxidant defences in blood by unknown mechanisms. More recently, studies using a liquid chromatography-MS-based metabonomic approach showed that lignins appear not to be metabolised by rats for $2 \mathrm{~d}$, but that they probably had some effects on endogenous metabolism ${ }^{(271)}$. To summarise, lignins might act in many ways: they are metabolised to enterolactone in rats $^{(221)}$, their antioxidant capacity may protect the gut epithelium, they may act on endogenous metabolism, they may reduce DNA damage in blood or cells via their antioxidant capacity and they may adsorb carcinogens. All these potential physiological effects should be taken into consideration in further in vivo studies, especially towards cancer prevention. Lignins are therefore far from being inert and researchers in nutrition and cereal technology should ask more questions about the nutritional effects of lignins.

The combined effects of $B$ vitamins. Whole-grain wheat, and especially its bran and germ fractions, contains almost all the $\mathrm{B}$-group vitamins, vitamins $\mathrm{B}_{1}$ (thiamin), $\mathrm{B}_{2}$ (riboflavin), $\mathrm{B}_{3}$ (niacin), $\mathrm{B}_{5}$ (panthothenic acid), $\mathrm{B}_{6}$ (pyridoxine), $\mathrm{B}_{8}$ (biotin) and $\mathrm{B}_{9}$ (folates). Whole wheat contains about $9.1 \mathrm{mg} \mathrm{B}$ vitamins $/ 100 \mathrm{~g}$, bran about $30.3 \mathrm{mg}$ and germ about $12.3 \mathrm{mg}$ (Table 1). Whole-grain cereals are particularly significant sources of thiamin, niacin, pantothenic acid and biotin compared with other food sources, and wheat germ is rich in nicotinic acid, pantothenic acid and pyridoxine. Cereal products are not a particularly rich source of folates unless fortified with folic acid (the synthetic form of folate), as it is often the case, especially for breakfast cereals. One key issue is the bioavailability of these vitamins in whole-grain cereals, but data are scarce: the few studies on the subject show that the bioavailability of each B vitamin seems to vary greatly, and that it is far from $100 \%$ (Table 2). Thiamin and pyridoxine are the most bioavailable (Table 2). The specific action of each of these vitamins is described in Table 3. Their actions are complex and multi-factorial. The $\mathrm{B}$ vitamins are also called the 'B-complex vitamins' and they play an important role in maintaining muscle tone in the gastrointestinal tract and promoting the health of the nervous system, skin, hair and liver. Thiamin, nicotinic acid, pyridoxine, pantothenic acid and folates play a positive role in mental health (Tables 3 and 4). For example, folates and pyridoxine are coenzymes in the one-carbon metabolism pathways and are involved in the synthesis of serotonin and other neurotransmitters, deficits of which are implicated in deficient mental health ${ }^{(272)}$. Folates also reduce the risk of neural tube defects in babies when consumed during the periconceptional period ${ }^{(273)}$. It was recently suggested that they could be used to treat depression ${ }^{(274,275)}$, as a low folate status is associated with depression ${ }^{(276)}$. Although difficult to demonstrate, it would be particularly interesting to explore the effect of whole-grain cereals on the nervous system and mental health, particularly disorders such as depression, insomnia, cognitive impairment or more generally psychic equilibrium. Other bioactive compounds, such as choline, ferulic acid, $\mathrm{Mg}, \mathrm{Zn}, \mathrm{Cu}$, inositols, policosanol and melatonin, are also potential candidates for mental health protection and equilibrium (Tables 3 and 4). 
The effects of whole-grain cereals on bone, teeth, articulation and tendon health. Whole-grain cereals and their fractions might contribute to the good health of bones, cartilages, teeth, collagen, joints and tendons (Table 3), which are all constituents of the skeleton, by the combined actions of $\alpha$-linolenic acid, $\mathrm{Fe}, \mathrm{Zn}, \mathrm{Mg}, \mathrm{Mn}, \mathrm{Cu}, \mathrm{P}, \mathrm{Ca}, \mathrm{K}$, nicotinic acid, tocotrienols, phylloquinone (vitamin $\mathrm{K}$ ) and $\beta$-cryptoxanthin (Table 4). While $\mathrm{P}$ and $\mathrm{Ca}$ are components of hydroxyapatite, a major constituent of bones and teeth, the $\mathrm{Ca}: \mathrm{P}$ ratio in cereals, notably wheat (about 0.08; Table 2), is below the ratio of $0 \cdot 5-0.8$ recommended for a satisfactory $\mathrm{Ca}$ use by the body. Ca from whole-grain cereals is therefore unlikely to contribute significantly to the health of bones and teeth. However, the addition of calcium carbonate $\left(\mathrm{CaCO}_{3}\right)$ to cereal food recipes before processing might be a simple way to achieve the desirable $\mathrm{Ca}: \mathrm{P}$ ratio without altering product palatability ${ }^{(277)}$. Whole-grain wheat also contains $\mathrm{Ca}$ absorption enhancers such as fructans and/or RS, which increase the apparent absorption of $\mathrm{Ca}$ from 20 to $50 \%$ in rats ${ }^{(71-73)}$. Similarly, inulin increases $\mathrm{Ca}$ absorption by about $12 \%$ in human subjects ${ }^{(71-73)}$. However, although whole-grain wheat does not contain inulin, it may contain up to $2.3 \mathrm{~g}$ fructans $/ 100 \mathrm{~g}$ (Table 2) that might also increase $\mathrm{Ca}$ absorption upon fermentation. The effect of indigestible oligosaccharides such as fructans on $\mathrm{Ca}$ absorption and metabolism, and bone health (as measured by indices such as bone mineral content and density, and/or bone resorption rate/osteopenia) is more and more recognised today, both in rats and humans ${ }^{(278-280)}$.

The results for $\mathrm{P}$ are less conclusive; some studies have shown increased $\mathrm{P}$ in bone following fructo-oligosaccharide consumption in rats ${ }^{(278,280)}$, while others have found no effect ${ }^{(280)}$. P is mainly supplied by phytic acid ( $>85 \%$ of the total $\mathrm{P}$ in grain), which has a high affinity for hydroxyapatite ${ }^{(281)}$. Indeed, the incidence of dental caries has been hypothesised to be concomitant with the change towards dietary habits of Western societies, as was shown with African Bantu acquiring susceptibility to dental decay as they adopted the European diet, through increased consumption of cariogenic refined foods such as refined sugar and white wheat bread in which a dominant cariespreventing factor would be removed during the refining process ${ }^{(282-284)}$. $\mathrm{P}$, which is abundant in less refined wheat flour, is involved in this effect ${ }^{(285)}$. Thereafter, several studies on rats using organic and inorganic phosphates and different $\mathrm{Ca}: \mathrm{P}$ ratios also showed the cariostatic effect of phytic acid ${ }^{(282,286-289)}$, possibly through its ability to affect organic materials and the adsorption of bacteria to tooth surfaces $^{(281)}$, and also through its ability to be rapidly adsorbed onto hydroxyapatite, forming a natural barrier resistant to acid attacks ${ }^{(290)}$ and thus to protect teeth from demineralisation and the formation of cavities by causing the desorption of salivary proteins from hydroxyapatite, the first step in plaque formation ${ }^{(281,291)}$. But, later, Cole \& Bowen failed to show a significant effect of feeding monkeys with phytic acid for 2 weeks on the physical properties of plaques (such as dry and wet weights), or their chemical properties (protein, carbohydrate, $\mathrm{Ca}, \mathrm{Mg}$ and $\mathrm{P}$ contents), or the microbial composition ${ }^{(292)}$. Further studies in human subjects are therefore needed to ascertain the cariostatic role of phytic acid, and perhaps of other cereal bioactive compounds, in subjects on a regular whole-grain cereal diet.

Whole-grain wheat also contains mammalian lignans $(0.2-0.6 \mathrm{mg} / 100 \mathrm{~g}$; Table 2) that seem to protect against osteoporosis (Table 3), notably in the postmenopausal period. Japanese women consuming high concentrations of phyto-oestrogens were found to have fewer hip fractures than women in the USA or Europe ${ }^{(293)}$. However, the effect of lignans on bone health remains to be confirmed. To my knowledge, no research has answered this particular issue of the role of long-term whole-grain cereal consumption on skeletal health and bone physiology.

Whole-grain cereals as a source of oligosaccharides. It has previously been seen that whole-grain cereals are rich in fibre (including RS) and oligosaccharides that may have both a prebiotic effect by favouring the development of a healthy microbiota ${ }^{294,295)}$ and that enhance mineral absorption through hypertrophy of the gut epithelium ${ }^{(70,71)}$. Thus, whole-grain wheat contains $1.9 \%$, its bran has $3.7 \%$ and the germ fraction $10.1 \%$ of fructans (fructooligosaccharide), raffinose and stachyose (Table 1). The average wheat germ raffinose content is about $8 \%$ and may reach $10.9 \%$, which is quite high (Table 2). Whole-grain wheat contains about $0.4 \%$ of raffinose and wheat bran has $1.2 \%$ (Table 2). The stachyose content is lower: $0.1 \%$ in whole-grain wheat, $0.2 \%$ in wheat bran and no data are available for wheat germ (Table 2). Raffinose is a trisaccharide composed of galactose, glucose and fructose. Stachyose is a tetrasaccharide formed with two galactose molecules, one glucose and one fructose. To my knowledge, there are no published data on the health effects of these whole-grain cereal oligosaccharides, apart from the fact that they are both considered to reinforce the fibre effect of whole-grain cereals, by producing SCFA generally favourable to large-bowel health. They are completely fermented in vitro within $48 \mathrm{~h}$ in the presence of a piglet faecal inoculum ${ }^{(296)}$. Rats fed a $3 \%$ raffinose-based diet for $21 \mathrm{~d}$ have a significantly reduced weight gain, more lactobacilli and fewer streptococci, greater SCFA production, and, interestingly, a lower plasma TAG concentration with no effect on plasma cholesterol ${ }^{(297)}$. However, it must be noted that fermented products (notably breads) constitute an important part of the whole-grain cereal food consumption of humans; and fermentation may lead to the partial breakdown of fructans, raffinose and stachyose by bacteria.

The specific action of phytosterol and of little studied bioactive whole-grain cereal compounds: $\alpha$-linolenic acid, policosanol, melatonin and para-aminobenzoic acid. The concentration of $\alpha$-linolenic acid, an $n$-3 fatty acid $(18: 3)$ with many positive health effects (Table 3 ), may reach $0.5 \%$ of wheat germ and almost $0.2 \%$ of wheat bran (Table 1 ). A diet containing about $2.7 \mathrm{~g} \alpha$-linolenic acid-rich wheat germ oil per $\mathrm{d}$ has an anti-atherosclerotic effect in mildly hypercholesterolaemic subjects; it acts by inhibiting oxidative stress-mediated synthesis of CD40L (protein involved in the progression of atherosclerosis with inflammatory and prothrombotic properties) ${ }^{(298)}$. Wheat 
germ contains $0.53 \% \alpha$-linolenic acid, so one should consume about $500 \mathrm{~g} / \mathrm{d}$ to reach the $2.7 \mathrm{~g}$ tested in the present study, which is not really realistic. However, a regular consumption of wheat germ as a nutritional complement and/or of wheat germ oil is nutritionally relevant.

Phytosterols, policosanol and melatonin, although present at lower concentrations, also possess numerous positive health effects (Table 3). Phytosterols, known for their cholesterol-lowering effect in humans ${ }^{(299,300)}$, are particularly high in wheat germ $(430 \mathrm{mg} / 100 \mathrm{~g}$ ) (Table 2) but their health effects are not known when they come from wholegrain cereals. Policosanol is a natural mixture of highmolecular-weight aliphatic primary alcohols (C24 to C34) in which octacosanol is the main compound ${ }^{(301,302)}$. Although less nutritionally studied, policosanol is also a lipid-lowering agent (for example, total and LDL-cholesterol) in both human subjects and animals at levels of about 10-20 mg daily, and it can also increase HDL-cholesterol up to $+30 \%{ }^{(303,304)}$, making it a promising agent in CVD prevention and treatment ${ }^{(304)}$. Whole-grain wheat contains about $3 \mathrm{mg}$ policosanol/100 g (Table 2). One recent study has shown that eating chocolate pellets supplemented with wheat germ policosanol $(20 \mathrm{mg} / \mathrm{d})$ for 4 weeks does not reduce blood cholesterol or modify the blood lipid profile of healthy human subjects ${ }^{(305)}$. A diet containing about $100 \mathrm{mg}$ policosanol/d eaten for $30 \mathrm{~d}$ reduced the increase in plasma LDL-cholesterol in hypercholesterolaemic rabbits by reducing cholesterol synthesis in the liver through increased $\mathrm{LDL}_{\text {catabolism }}{ }^{(306)}$. Feeding policosanol to rats for up to 4 weeks ( 250 and $500 \mathrm{mg} / \mathrm{kg}$ per d) significantly renders the lipoprotein fractions (VLVL + LDL) resistant to ex vivo $\mathrm{Cu}$-mediated oxidation ${ }^{(307)}$. In view of these results, the policosanol content of whole-grain wheat seems too low (about $3 \mathrm{mg} / 100 \mathrm{~g}$ ) to significantly improve the blood lipid profile in humans. Rather, it is probably the combined action of the different cholesterol-lowering compounds of wheat (for example, SCFA produced by undigestible carbohydrates, soluble fibre, tocotrienols, phytosterols and policosanol) that contributes to improve the blood lipid profile to its optimum.

The concentration of the mammalian pineal hormone melatonin, which can be extracted from numerous plants, is about $0.3 \mu \mathrm{g} / 100 \mathrm{~g}$ in whole-grain wheat (Table 2) ${ }^{(308)}$. This compound has a positive effect on human mood, cognitive functions, prolonged sleep period and brain neuromodulation $^{(309,310)}$, but it may also be an antioxidant $^{(310)}$ and anti-carcinogen ${ }^{(311,312)}$ (Table 3). The health effects of melatonin in humans when originating from whole-grain cereals are not known: as for policosanol and other cholesterol-lowering compounds, due to the low melatonin content of whole-grain wheat (Table 4), this is probably the combined action of melatonin and of other compounds acting positively on mental and brain health that has to be considered first.

Para-aminobenzoic acid has also been detected in cereals. Values are scarce and not recent: reported values are $0.34-0.55,1.34$ and $0.852 \mathrm{mg} / 100 \mathrm{~g}$ for whole-grain wheat, bran and germ fractions, respectively ${ }^{(313,314)}$. Paraaminobenzoic acid is best known as a sunscreen agent that protects the skin from UV radiation ${ }^{(315)}$, but it also stimulates bacterial growth in the intestine and is an intermediate in the bacterial synthesis of folates. Besides its role in folate formation, para-aminobenzoic acid has long been used to treat rickettsial infections and may lead to a $11.5 \%$ decrease in serum cholesterol in man, when consumed at $8 \mathrm{mg} / \mathrm{d}$ in the form of its Na salt ${ }^{(316,317)}$. Paraaminobenzoic acid down-regulates $N$-acetyltransferase in human cell cultures (peripheral blood mononuclear cells) $)^{(318)}$ - acetylation plays an important role in the activation of several potential human carcinogens ${ }^{(319,320)}$, and inhibits the production of thromboxane which participates in blood coagulation (anti-aggregatory effect) and in increased arterial pressure through vasoconstriction ${ }^{(321)}$. However, these studies used para-aminobenzoic acid concentrations of $30-100 \mu \mathrm{M}$, about $4-137 \mathrm{mg} / \mathrm{l}$, which is far higher than the quantity that can be obtained from eating whole-grain cereal products, as whole-grain wheat containing only $0.34-0.55 \mathrm{mg}$ para-aminobenzoic acid/ $100 \mathrm{~g}$ (Table 2). Thus, like the other bioactive compounds present at low concentrations in whole-grain wheat (for example, policosanol and melatonin), the health benefit of cereal para-aminobenzoic acid has to be considered complementary to that of other cholesterol-lowering, anti-carcinogenic and anti-aggregatory compounds.

\section{The nutrigenomic approach}

Nutrigenomics in nutrition is devoted to the study of the influence of dietary interventions on gene transcription (transcriptome), protein synthesis (proteome) and metabolites (metabolome, the whole set of metabolites) in cells, body fluids and tissues ${ }^{(322-326)}$. One of the most important objectives of nutrigenomics is to detect and identify early metabolic disturbances and their regulation (for example, in relation to oxidative stress or inflammation) that can lead to more serious chronic diseases. The possibility of detecting some diseases early could change clinical nutrition and public health practices ${ }^{(326)}$. This implies studying the effects of bioactive compounds in whole-grain cereals on gene expression, protein synthesis and the metabolome. In the field of nutritional studies, besides the measurement of usual biomarkers such as plasma glucose (for example, GI) or urinary lipid peroxides (oxidative stress index), it seems particularly important to focus on the metabolome, which reflects both the endproducts of metabolism and the changes over time of metabolism following food consumption. While many metabolomic studies have been done with isolated compounds, notably in pharmacology for drug toxicity ${ }^{(327)}$, very few have been done with complex food products. In metabolomics and nutrition, only a few studies have been performed ${ }^{(328)}$ : to characterise the metabolic effect of energy restriction ${ }^{(329)}$, vitamin deficiency ${ }^{(330)}$ or of intake of PUFA-rich oils ${ }^{(331)}$, antioxidant-rich foods such as soya ${ }^{(332)}$, chamomile ${ }^{(333)}$ and tea ${ }^{(334)}$, or of pure dietary antioxidants such as epicatechin (335), catechin $^{(336)}$ or ferulic and sinapic acids and lignins ${ }^{(271)}$. Studies on rats have been carried out using the metabolomic approach to explore the metabolic fate and the effect on endogenous metabolism of whole-grain and refined wheat flours ${ }^{(230)}$ and of ligninenriched wheat bran lignins ${ }^{(271)}$. It has thus been shown that whole-grain wheat flour consumption leads to significant increases in liver betaine and GSH and decreases in some 
liver lipids, but has no effect on conventional lipid and oxidative stress biomarkers. It also causes a greater urinary excretion of tricarboxylic acid cycle intermediates, aromatic amino acids and hippurate (from phenolic acid degradation in the colon). When the diet was changed to refined wheat flour, a new metabolic balance was reached within $48 \mathrm{~h}$, and conversely from refined to whole-grain flour (Fig. 3) ${ }^{(230)}$. The metabolomic approach also showed that rats did not appear to metabolise lignins from wheat bran within $2 \mathrm{~d}$ of the regimen, but they are likely to affect endogenous metabolism through mechanisms which need to be elucidated ${ }^{(271)}$. Results are convincing in that new metabolic effects have been unravelled using this new open approach, for example, the role of symbiotic microbiota in triggering diet-induced mechanisms of steatosis ${ }^{(337)}$ or some specific metabolic pathway disturbances in diabetic rats ${ }^{(338)}$, thus improving our understanding of diseases and the mechanisms responsible for them. However, more significant conclusions could be drawn once the databases for compound identification are completed and distributed. To my knowledge, few if any studies have investigated the effect of consuming complex whole-grain cereals and their fractions on gene expression. The tools are now available to study this, which would provide important information about which gene-regulated metabolic pathways are stimulated by the synergetic action of the bioactive compounds in whole-grain cereals, not the restricted action of isolated compounds. Thus, nutrigenomics should enable us to better characterise the metabolic pathways affected in vivo by the antioxidants in whole-grain cereals.

\section{Conclusion}

The metabolic fate and health effects of major compounds such as lignin (up to $9 \%$ in wheat bran), ferulic acid (up to $0.6 \%$ in wheat bran), phytic acid (up to $6 \%$ in wheat bran) and betaine (up to $1.5 \%$ in wheat bran) (Table 2) have been little studied when originating from whole-grain cereals. Yet, these three compounds may account for about $11 \%$ of wheat bran (Table 1), and therefore deserve to be studied more. Wheat germ also merits greater attention since it contains quite significant levels of bioactive compounds such as $\alpha$-linolenic acid (about $530 \mathrm{mg} / 100 \mathrm{~g}$ ), GSH (about $133 \mathrm{mg} / 100 \mathrm{~g}$ ), GSSG (about $69 \mathrm{mg} / 100 \mathrm{~g}$ ), thiamin (about $1.75 \mathrm{mg} / 100 \mathrm{~g}$ ), vitamin $\mathrm{E}$ (about $27.1 \mathrm{mg}$ total tocols/ $100 \mathrm{~g}$ ), flavonoids (about $300 \mathrm{mg} / 100 \mathrm{~g}$ ), betaine (about $851 \mathrm{mg} / 100 \mathrm{~g}$ ), choline (about $223 \mathrm{mg} / 100 \mathrm{~g}$ ), myo-inositol $(>11 \mathrm{mg} / 100 \mathrm{~g}$ ) and phytosterols (about $430 \mathrm{mg} / 100 \mathrm{~g}$ ) (Table 2). It thus contains $2.5 \%$ of vitamins and minerals, at least $1.6 \%$ of lipotropic compounds and $1.2 \%$ of sulfur compounds. All these compounds are involved in the new hypotheses proposed here and their corresponding physiological mechanisms. Based on past and new hypotheses, a synthetic view of the mechanisms underlying the health benefits of whole-grain cereals and their fractions can be proposed (Fig. 4). The diagram purposefully illustrates the complexity of the mechanisms involved and their obvious synergy and interconnection in vivo. Due to this complexity, whole-grain cereal bioactive compounds are listed in Table 4, ranking according to the five major health outcomes generally considered in the literature: body-weight regulation, CVD, diabetes, cancers, and gut health; mental, brain

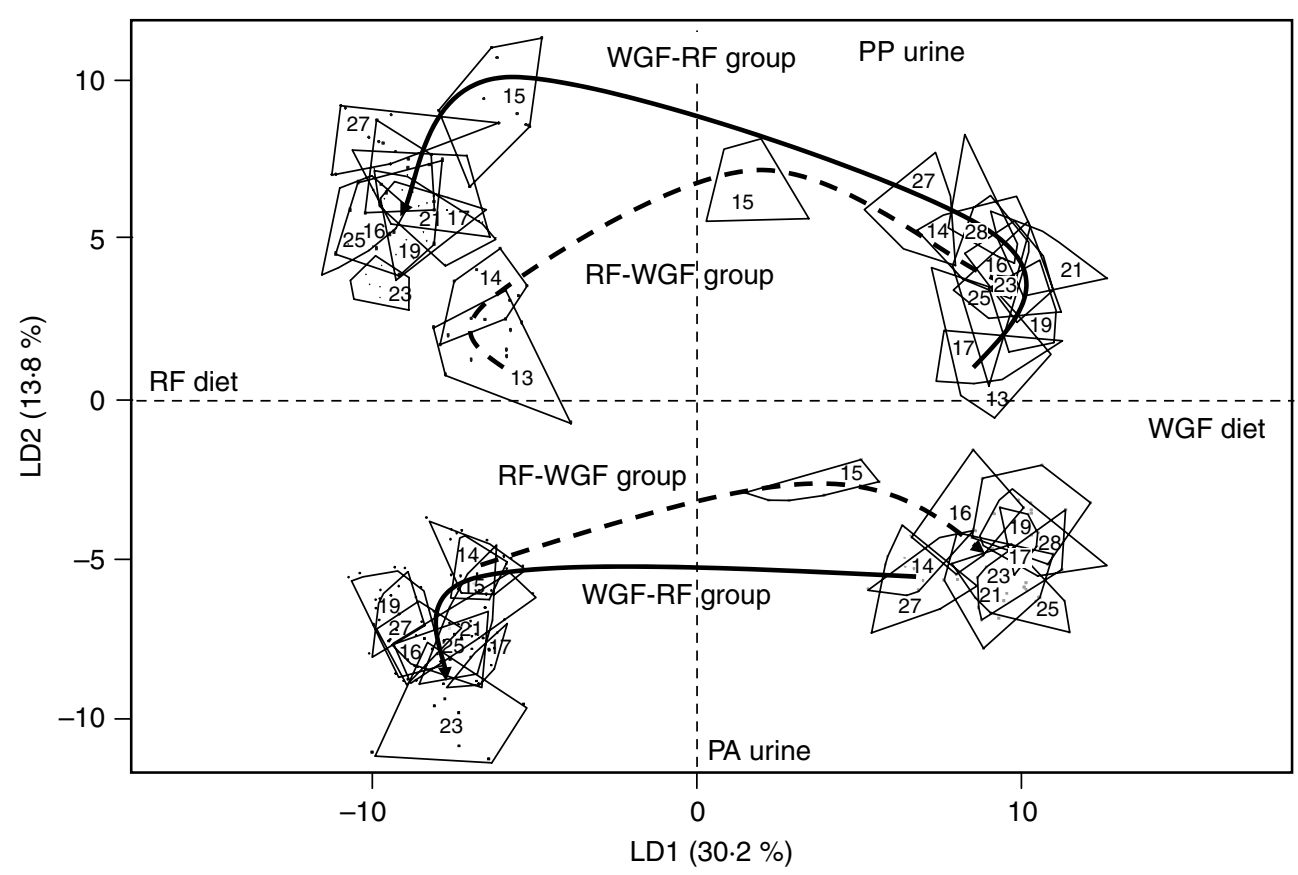

Fig. 3. Linear discriminant (LD) analysis score plot of the ${ }^{1} \mathrm{H}$ NMR urinary spectra highlighting the separation before, between and after the diet change (days 14-15) and between the urine sampling times (postprandial (PP) and post-absorptive (PA)). (- - --), Refined flour followed by wholegrain flour consumption (RF-WGF) group; (-), whole-grain flour followed by refined flour consumption (WGF-RF) group. Each polygon represents the limits of the metabolic profile obtained for the ten rats of a given group at a given day and urine sampling time. Urine samples were collected from days 13 to 28 (for details, see Fardet et al. ${ }^{(230)}$ ). 


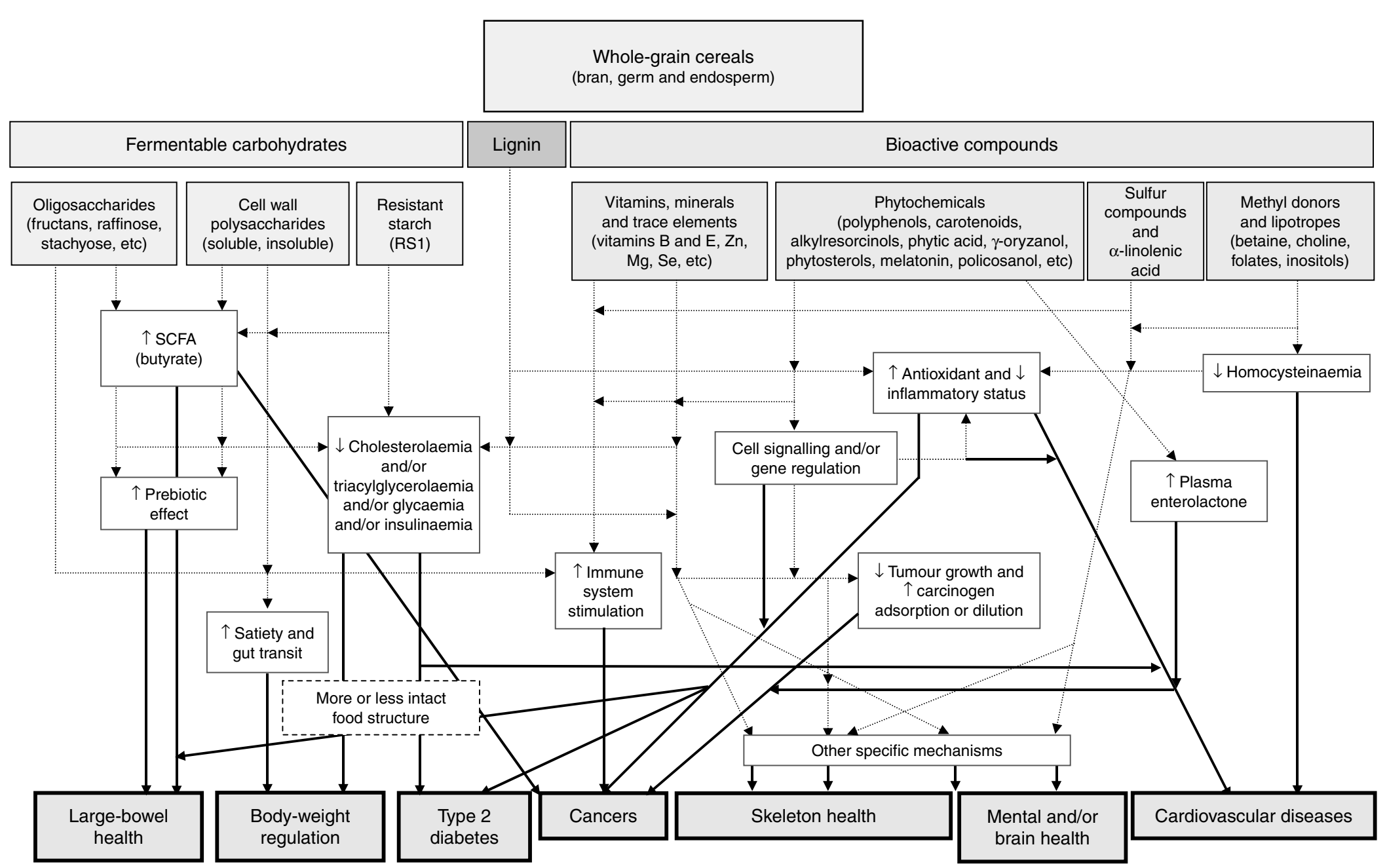

Fig. 4. Current and new proposed physiological mechanisms involved in protection by whole-grain cereals (adapted from Table 3). The dotted thin arrows (-....) indicate the link between wholegrain bioactive compounds and protective physiological mechanisms, while the plain arrows $(\rightarrow)$ indicate the relationship between physiological mechanisms and health outcomes. 
and skeleton health being new proposed ways to explore. One important question remains: do bioactive compounds exert the same effects when they are free compounds and when they are in whole-grain cereals? This is notable, because their bioavailability in whole-grain cereals is probably lower than the free compounds (Table 2) and because the quantities in whole-grain cereal products do not match the daily human needs. Again, it is probably the summed and combined action of all the bioactive compounds on a particular physiological function (as illustrated in Fig. 4 and Table 4) which leads to improved specific physiological functions such as antioxidant status and glucose homeostasis, especially when whole-grain products are consumed daily, generating long-term health benefits. This is why it is urgent to carry out further in vivo studies both in rats and human subjects, to unravel the complex mechanisms activated by the consumption of highly complex foods such as whole-grain cereal products. Intervention studies on human subjects consuming wholegrain cereals are so rare that they should be carried out first. The non-invasive characteristic and high potential of the metabolomic approach for unravelling new metabolites and metabolic pathways affected by a given diet and its ability to explore the complexity inherent in metabolism means that it should accompany the measurement of the usual biomarkers in order to describe the metabolic actions of whole-grain cereals in all their complexity. The mechanisms described in Fig. 4 are complex, but are above all interconnected as in the whole organism. Metabolomics therefore seems to be the most appropriate tool for studying such an interconnectedness, and so provide a more realistic view of how wholegrain cereal bioactive compounds act in synergy. For example, inflammation, oxidative stress and immune system-related metabolic pathways are generally all involved in cancers, as is the case for other metabolic diseases in which there is a progressive metabolic imbalance following an unhealthy diet. Finally, genomic studies are needed on the action of whole-grain cereals on gene regulation, as bioactive compounds really exert their physiological effects within the cell. While isolated free bioactive compounds may be used for in vitro studies on cell cultures, studies in animals and human subjects should use an integrated 'complex food approach'.

\section{Cereals other than wheat}

The present review discusses whole-grain wheat, since it is one of the most widely consumed cereals, especially in Western Europe. However, most of the bioactive compounds in wheat are also present in other major cereals such as rice, maize, oats, barley, sorghum and millet. The main differences lie in the relative contents of each of these compounds, their distribution in bran, germ and endosperm and the proportions of the bran and germ fractions. Nevertheless, compounds such as $\gamma$-oryzanol, avenanthramides and saponins are specific to cereals other than wheat.

\section{The bran fraction}

The proportion of the bran fraction varies with the cereal type: for wheat, rice and maize, it is $10-16 \%$ of the whole grain. The bran fraction in rice contains about 15-20\% $\mathrm{oil}^{(215,339)}$. This oil is rich in bioactive compounds and contains more than 100 different antioxidants, such as lipoic acid, a powerful antioxidant ${ }^{(340,341)}$ that helps prevent cognitive deficits, is beneficial in the treatment of Alzheimer's disease ${ }^{(342)}$, and may protect against risk factors of $\mathrm{CVD}^{(343)}$. Rice bran contains tocotrienols $(10.6 \mathrm{mg} / 100 \mathrm{~g})^{(344)}, \gamma$-oryzanol $(281 \mathrm{mg} / 100 \mathrm{~g})^{(344)}$ and up to $1.2 \%$ phytosterols ${ }^{(345)}$ such as $\beta$-sitosterol, all of which may help improve the blood lipid profile and reduce the risk of $\mathrm{CVD}^{(346-348)}$. Rice bran also contains up to $21 \%$ dietary fibres ${ }^{(345)}$. Maize bran has more dietary fibre than wheat and rice bran, about $74-79 \%(215,349,350)$. It contains about $4 \%$ phenolic acids, about $50 \%$ heteroxylans and about $20 \%$ cellulose, and is almost devoid of lignins ${ }^{(350)}$. It is particularly rich in ferulic acid (up to $3 \%$ ), mainly in a very resistant (to enzymes) bound form ${ }^{(351)}$. And, contrary to wheat for which phytate is essentially in the bran fraction, $90 \%$ of maize phytate is in the germ fraction ${ }^{(352)}$.

\section{Some specific compounds}

Some bioactive compounds are quite specific to certain cereals: $\gamma$-oryzanol in rice, avenanthramides and saponins in oats, and, although present in other cereals such as wheat, $\beta(1 \rightarrow 3)(1 \rightarrow 4)$-glucans in oats and barley, and alkylresorcinols in rye. Their mechanisms of action and health effects are shown in Table 3.

$\gamma$-Oryzanol in rice. $\gamma$-Oryzanol is derived from rice bran oil and is a mixture of substances including sterols and ferulic acid, and at least ten phytosteryl ferulates (for example, methylsterols esterified to ferulic acid). Its content in whole-grain rice is $18-63 \mathrm{mg} / 100 \mathrm{~g}(\mathrm{DW})^{(339,353)}$ and in rice bran $185-421 \mathrm{mg} / 100 \mathrm{~g}$, depending on the rice variety, milling time, stabilisation process and extraction methods ${ }^{(344,354-356)}$. Its antioxidant activity has been demonstrated in vitro ${ }^{(357)}$. Its health effects are diversified, with positive actions against CVD and hyperlipidaemia, as shown in animal models through cholesterol-lowering, lipid peroxidation reduction and anti-atherogenic effects $^{(348,358-360)}$ and in human subjects ${ }^{(361)}$.

Avenanthramides and saponins in oats. Aventhramides are specific polyphenols from oats. They are substituted cinnamic acid amides of anthranilic acids and there are at least twenty-five distinct entities ${ }^{(362)}$. Total avenanthramide content in five oat cultivars (husked and naked) ranges from 4.2 to $9.1 \mathrm{mg} / 100 \mathrm{~g}^{(363)}$, while the oat grain contains 4-13 mg avenanthramide $1 / 100 \mathrm{~g}$ (the major avenanthramide), again depending on the oat cultivar ${ }^{(364)}$. The avenanthramide content in oat bran is $1.3-12.5 \mathrm{mg} / 100 \mathrm{~g}$ according to the type of avenanthramide considered ${ }^{(364,365)}$. As polyphenols, they are strong antioxidants both in vitro ${ }^{(366,367)}$ and in vivo ${ }^{(140)}$. They play a particular role in the prevention of CVD due to their anti-inflammatory and anti-atherogenic effects $^{(368)}$, and by protecting LDL from oxidation, in synergy with vitamin $\mathrm{C}$, as shown on human $\mathrm{LDL}^{(369)}$.

Saponins are glycosides with a steroid or triterpenoid aglycone ${ }^{(370)}$. They are especially found in oats, which synthesise two families of saponins, the steroidal 
avenacosides and the triterpenoid avenacins ${ }^{(371)}$. The saponin content, depending on the oat cultivar, seems to be situated mainly within the endosperm and has been shown to vary from 0.02 to $0.13 \%(\mathrm{DW})^{(372,373)}$. Saponins have a wide range of biological activities (about fifty are listed by Güçlü-Üstündag \& Mazza ${ }^{(370)}$, , such as anticarcinogenic and hypocholesterolaemic ${ }^{(374)}$, stimulation of the immune system $^{(375,376)}$ and cholesterol-lowering ${ }^{(377)}$. However, it is not known whether all these properties could be ascribed to cereal saponins. Saponins are also poorly absorbed by the gut ${ }^{(267)}$.

$\beta(1 \rightarrow 3)(1 \rightarrow 4)$-Glucan in barley and oats. The $\beta(1$ $\rightarrow 3)(1 \rightarrow 4)$-glucan content of oats and barley is especially high. Total, insoluble and soluble barley $\beta$-glucan contents vary widely with the variety, the presence of hull (i.e. hulled v. hull-less) and the amylose content ${ }^{(378)}$. Thus, the watersoluble $\beta$-glucan content of barley is $0.5-8.3 \%(\mathrm{w} / \mathrm{w}$, $\mathrm{DW})^{(378-385)}$, the insoluble fraction is $1.2-21.7 \%(\mathrm{w} / \mathrm{w}$, DW $)^{(379-381)}$ and the total $\beta$-glucan content is $3 \cdot 0-27 \cdot 17 \%$ $(\mathrm{w} / \mathrm{w}, \mathrm{DW})^{(379-381,383)}$. Total $\beta$-glucans contents vary widely and might be attributable, in addition to variety variability, to the method of extraction and possible confusion in some studies where the soluble $\beta$-glucan fraction seems to be confounded with the total $\beta$-glucans.

The soluble $\beta$-glucans content of naked oat grains is $3.9-7.5 \%$, and in hulled oat grains it is $2.0-7.5 \%(\mathrm{w} / \mathrm{w}$, DW); the insoluble content of naked oat grains is $5.2-10.8 \%$ and that of hulled oat grains is $13.8-33.7 \%$ $(\mathrm{w} / \mathrm{w}, \mathrm{DW})^{(381,386)}$. Much work has already been done on the health effects of $\beta$-glucans, particularly their glycaemiaand cholesterol-lowering properties, having implications for type 2 diabetes $^{(387)}$ and CVD $^{(56,388,389)}$. As soluble viscous fibre $^{(383)}$, they slow the rate of gastric emptying, and the diffusion of glucose and NEFA into epithelial cells for absorption in both animals and humans ${ }^{(56,389)}$. However, a recent study conducted on healthy subjects demonstrated that muesli enriched with oat $\beta$-glucans had no more effect on gastric emptying rate than did cornflake-based muesli, despite its plasma glucose-lowering effect ${ }^{(390)}$. $\beta$-Glucans are also positively involved in the protection against cancers, especially through reactions with mutagenic agents to prevent them interacting with DNA as shown in rodent and human cell lines ${ }^{(391)}$.

Alkylresorcinols in rye. Alkylresorcinols are plant-derived phenolic lipids, especially found in whole-grain cereals. Rye contains the highest concentration of alkylresorcinols, which can be twice that of wheat (up to $320 \mathrm{mg} / 100 \mathrm{~g} \mathrm{DW}$ ) ${ }^{(392)}$. They are 1,3-dihydroxybenzene derivatives with an alkyl chain at position 5 of the benzene ring, which gives them an amphiphilic feature. They are apparently relatively well absorbed within the small intestine (about 58\%; Table 2) of ileostomates following the consumption of soft bread enriched with rye bran and whole-grain rye crispbread ${ }^{(393)}$, making them (either intact in plasma or as metabolites in urine) potential biomarkers of whole-grain rye and wheat intake ${ }^{(394-396)}$, especially for epidemiological research and observational studies ${ }^{(396,397)}$. Their biological activity is multifactorial $^{(396)}$, from interacting with metabolic enzymes (for example, inhibiting 3-phosphoglycerate dehydrogenase, the key enzyme in TAG synthesis in adipocytes) ${ }^{(398)}$ to decreasing cholesterol in the rat liver ${ }^{(399)}$, to anticancer/ cytotoxic effects but almost exclusively in vitro ${ }^{(400,401)}$.

\section{New bases for improving the nutritional properties of cereal products}

The elucidation of the mechanisms by which whole-grain cereals protect our bodies, together with a better understanding of how bioactive compounds are released from the cereal food matrix and delivered to the bloodstream, will provide important information for the industrial development of cereal products with improved nutritional qualities. Surprisingly, the present supply of cereal products of a good nutritional quality is still limited. I believe that the best way to improve the nutritional quality of cereal products is to combine the preservation of a relatively intact botanical food structure (as far as the recipe allows it), a low-GI feature and a high nutritional density of fibre and bioactive compounds, by using less refined flour with a higher extraction rate. These factors are important but probably not sufficient to ensure that the right macro- or micronutrient reaches the right site of absorption for an optimal physiological effect. This is why more and more private and public research is aimed at modelling the fate of nutrients from complex foods within the intestine so as to predict their bioaccessibility and thus control their delivery for a specific physiological effect ${ }^{(402-404)}$.

\section{Optimising and controlling the delivery of bioactive compounds for improving health}

There are great differences between the food content in a defined nutrient and the percentage really metabolised, or even absorbed. This is especially true for cereal products where numerous factors linked to the food matrix may limit the release of macro- and micronutrients. There is increasing evidence that the physical structure of natural cereal food matrices (for example, intact cereal kernels) or the artificial microstructure of processed cereal products may either favour or limit the bioavailability of nutrients, and thus their nutritional effects. However, differences in bioaccessibility-bioavailability of nutrients, particularly micronutrients, at present cannot be correlated with differences in long-term health effects, except for the positive health effects of starch and its so-called slowly digestible fraction ${ }^{(405,406)}$. The question is therefore: is there a positive correlation between increased or decreased bioaccessibility of a given nutrient and its health effect? This probably depends on the nutrient considered and on the health status of the subject. For example, the rapid release of glucose from starch digestion into the bloodstream is advantageous in some situations (for example, the urgent need for glucose for brain or muscles to function, as for immediate intellectual and physical efforts), and harmful in other situations (for example, type 2 diabetes). The same approach is now being developed for proteins (slow $v$. rapid proteins) and lipids for which their physical state and/or their physico-chemical properties may influence the release of amino acids and fatty acids, respectively, into the bloodstream. The resulting significant metabolic impact 
could be used in some situations such as diabetic subjects $^{(407)}$, the elderly ${ }^{(408)}$ and for patients on enteral nutrition suffering from pancreatic insufficiency to adequately hydrolyse lipids ${ }^{(409)}$.

In vitro bioaccessibility and in vivo bioavailability studies with vegetables and whole-grain cereals and/or their fractions have clearly shown that food structure affects the bioavailability of polyphenols, carotenoids, minerals, trace elements and vitamins (Table 2) ${ }^{(403)}$. Table 2 shows the results of bioavailability studies on whole-grain wheat products and wheat bran. Much data are still lacking: studies exploring the bioavailability of compounds in whole-grain cereals are scarce and the products are often consumed as part of a complex diet that also supplies the same bioactive compounds from other foods. For example, studies on mineral or trace element bioavailability in rats often included mineral mixtures that made it difficult to determine the exact apparent absorption of the mineral supplied by the cereals. Thus, radiolabelled cereal products should be used more frequently to answer such questions. The few data obtained show that bioactive compounds are far from being $100 \%$ bioavailable within the small intestine. No more than $5 \%$ of the ferulic acid in wheat bran is released into the small intestine, so that most reaches the colon where it can exert an antioxidant protective action on the gut epithelium. On the other hand, there is convincing evidence that the small proportion absorbed in the small intestine can affect cell signalling and the activation or repression of some genes. Thus, in a way similarly to starch, it seems that two fractions of ferulic acid can be defined: the rapidly available ferulic acid released and absorbed in the small intestine (i.e. free and soluble-conjugated), and slowly available ferulic acid gradually released mainly in the colon (i.e. ester-linked) ${ }^{(264)}$, each fraction having its own health benefits.

Betaine (about $0.9 \%$ of wheat bran; Table 1), unlike ferulic acid, is probably much more bioavailable since it is not bound to other constituents: is there a need to slow down its release and to favour a fraction reaching the colon, for example, for improving its anticancer effect ${ }^{(410)}$ ? The same issue, that is the optimal bioavailability to reach, might be questioned for polyphenols such as lignans and alkylresorcinols, vitamins and minerals, and phytosterols. The problem for phytic acid is slightly different; we need to know the extent to which it is reasonable to pre-hydrolyse it in order to combine a maximum mineral bioavailability with its antioxidant effect in the gut against free radicals produced by microbiota, and from its potential hypoglycaemic effect as well.

Otherwise, the case of fibre is not yet resolved for wholegrain wheat which contains more insoluble fibre than soluble fibre (soluble:total fibre ratio is about $0 \cdot 16$; calculated from Table 2): what would be the optimum ratio of soluble:total fibre to reach? It is not known to what extent it would be beneficial to increase the soluble fibre content, for example, by pre-hydrolysing insoluble arabinoxylans to soluble arabinoxylans (soluble:total arabinoxylans ratio is about 0.18 ; calculated from Table 2). Soluble fibres may be beneficial to health by reducing the postprandial glucose response through increased viscosity ${ }^{(411)}$ (see Tables 3 and 4 ), but they may also be harmful, by, for example, increasing the risk of colon cancer ${ }^{(412)}$.
Provided it has positive health benefits, the range by which industrial processes can improve the bioaccessibility and bioavailability of cereal bioactive compounds is therefore large. This approach has been applied to starch with success ${ }^{(413)}$, by controlling its delivery in the gut by rendering it more slowly hydrolysed (i.e. slowly digestible starch) within the small intestine, or by making it inaccessible to $\alpha$-amylase (i.e. RS), so that a fraction of starch reaches the colon where it is fermented to the anticarcinogenic molecule butyrate, the preferred fuel for colonocytes (see Whole-grain cereals and butyrate production section). Technologists know how to modulate the proportions of these three fractions in cereal products, i.e. rapidly, slowly and indigestible starch. RS is representative of the different ways it can be used by breeders and technologists to control the delivering of a compound, i.e. starch, within the digestive tract. It has been seen that the RS content of whole-grain products may be very high, up to $12 \%$ in ordinary barley kernels and even $22 \%$ by combining intact botanical structure with a high-amylose barley variety $^{(54)}$. The formation of RS can be technologically favoured through starch encapsulation within the cereal food matrix by protein or fibre networks (RS1), restricting starch granule gelatinisation (RS2), the use of high-amylose cereal varieties with a high content of retrograded starch (RS3) and/ or chemical modification such as acylation (RS4). RS is now considered to be a prebiotic compound that can positively modify microbiota growth in quality and quantity within the colon $^{(414,415)}$. If technologists may be able to modify processing parameters such as temperature, extrusion pressure, retrogradation and/or chemical modification to increase the RS content, breeders can select high-amylose cereal varieties ${ }^{(294,416)}$, amylose being more slowly digested than amylopectin ${ }^{(417,418)}$.

\section{The traditional use of fermentation and the development of new technologies}

Fig. 5 shows the ways in which the nutritional quality of whole-grain cereals can be improved. There are mainly three: the growing conditions, the genetic approach and through technological processes.

Growing conditions. The growing conditions, for example, the use of adequate fertilisers, can increase the cereal content of $\mathrm{Se}, \mathrm{Mg}, \mathrm{Fe}$ and $\mathrm{Zn}^{(419-421)}$ with possible modified physiological effects in humans ${ }^{(422)}$. An increase in environmental stress, for example, water stress, cold or exposure to micro-organisms, may favour the synthesis of antioxidants by the plant to combat this stress. This has been shown with $\alpha$-tocopherols, carotenoids and betaine in wheat seedlings and sugarbeet roots under temperature- and salt-stressed environments ${ }^{(423,424)}$

Genetic approach. The genetic approach ${ }^{(425)}$ using conventional tools (indirect action on genes) such as cross-breeding and hybridisation to combine varieties high in some bioactive compounds, for example, $\mathrm{Zn}, \mathrm{Fe}$ and pro-vitamin $\mathrm{A}^{(426,427)}$, and/or low in others, for example, phytic acid ${ }^{(428,429)}$, and non-conventional tools (direct action on genes) such as genetic engineering to modify 


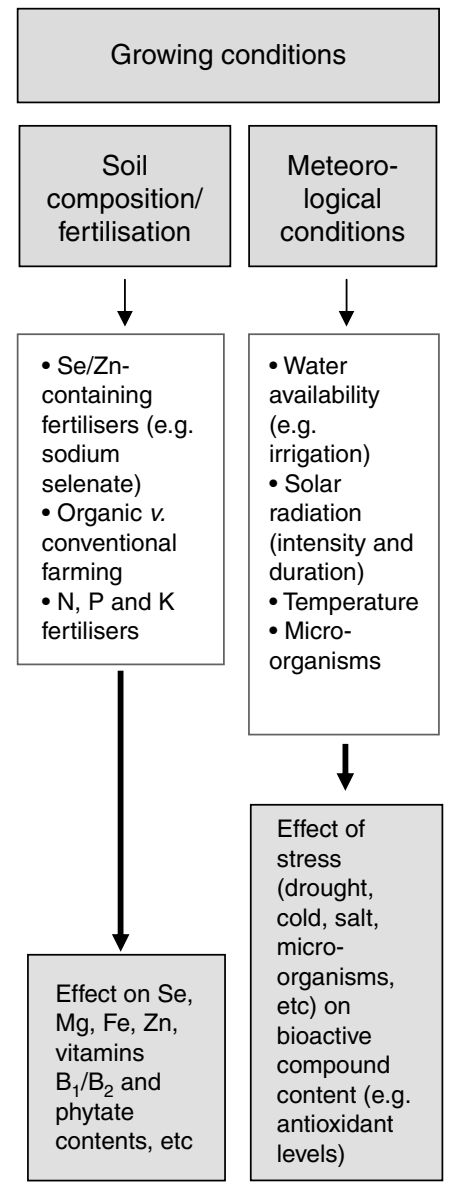

\begin{tabular}{|c|c|c|}
\hline \multicolumn{3}{|c|}{ Genetics } \\
\hline $\begin{array}{l}\text { Micronutrient- } \\
\text { dense species } \\
\text { selection }\end{array}$ & $\begin{array}{l}\text { Cross- } \\
\text { breeding and } \\
\text { hybridisation }\end{array}$ & $\begin{array}{l}\text { Genetic } \\
\text { engineering }\end{array}$ \\
\hline$\downarrow$ & $\downarrow$ & $\downarrow$ \\
\hline $\begin{array}{l}\text { Different species, } \\
\text { e.g. } \\
\text { - Wild and primitive } \\
\text { wheats } \\
\text { - Durum v. soft } \\
\text { wheats } \\
\text { - Pigmented wheat } \\
\text { - Diploid } v \text {. } \\
\text { tetraploids }\end{array}$ & $\begin{array}{c}\text { Combining } \\
\text { different strains } \\
\text { of a species or } \\
\text { members of } \\
\text { different } \\
\text { species }\end{array}$ & $\begin{array}{l}\text { GM species: } \\
\text { - Gene silencing } \\
\text { through RNA } \\
\text { interference } \\
\text { • Gene splicing } \\
\text { through } \\
\text { recombinant } \\
\text { DNA carrying } \\
\text { reporter gene }\end{array}$ \\
\hline \multicolumn{3}{|c|}{$\begin{array}{l}\text { Variability in the bioactive compound contents according to } \\
\text { cereal varieties and genotypes selected (e.g. see ranges in } \\
\text { Table } 2 \text { for wheat) } \\
\text { Selection, combination and addition of specific traits: } \\
\text { - High amylose content } \rightarrow \text { increases RS content } \\
\text { - Increase in mineral and/or vitamin contents (provitamin A) } \\
\text { - Increase in arabinoxylan or } \beta \text {-glucan contents } \\
\text { - Protein/amino-acid contents of the aleurone layer } \\
\text { - Low-phytic acid mutants } \rightarrow \text { improve mineral bioavailability } \\
\text { - High phytase varieties or over-expression of phytase } \\
\text { - Low-protease inhibitor variety }\end{array}$} \\
\hline
\end{tabular}

\begin{tabular}{|l|}
\hline \multicolumn{2}{|c|}{ Technological processes } \\
\hline \multicolumn{1}{|c|}{ Food shaping and cooking procedures } \\
\hline Milling
\end{tabular}

Fig. 5. Ways for improving cereal product nutritional quality. RS, resistant starch. 
gene expression in relation to the nutrient synthesis and/or metabolism can be used to improve the nutritional quality of whole-grain cereals. By these means, the amylose $^{(294,430,431)}, \mathrm{RS}^{(416)}$, arabinoxylan ${ }^{(432)}$ and mineral/vitamin $^{(419,433)}$ contents can be modified (i.e. increased in most cases).

Development of new technologies. Besides growing conditions and genetics, the third way of improving the nutritional quality of cereal products is through technological processes. The literature about them is plethoric, but it is not an objective of the present paper to review them. However, some key issues may be emphasised since they allow optimising the health benefits of cereal by preserving their nutritional density and food structure.

Increasing nutritional density in bioactive compounds through germination, soaking and pre-fermentation of whole-grain cereals and/or their fractions. Cereals are usually processed in two main ways. The first is dry fractionation followed by cooking under different conditions of water content, temperature and pressure, as for pasta, biscuits, breakfast cereals and other cereal products widely consumed in Western countries. The second is fermentation. This is generally used for whole-grain cereals in more traditional procedures used for the many wholegrain foods consumed in developed countries and several alcoholic beverages (for example, beer, sake, whisky, etc) consumed around the world ${ }^{(434,435)}$. A fermentative step stimulates enzyme activities, which generally increases the content of free bioactive compounds. Bread products combine both approaches by using dry milling, fermentation and cooking.

Due to the plasma cholesterol- and glucose-lowering properties of soluble fibre and to its low content in wheat, due to the numerous health effects of free ferulic acid ${ }^{(261,262)}$, and due to the relative negative effect of phytic acid upon mineral bioavailability $^{(217)}$, different ways to pre-hydrolyse insoluble fibre (for example, insoluble $\beta$-glucans or arabinoxylans) into soluble fibre with endohydrolases ${ }^{(150,436)}$, ester-bound ferulic acid into free ferulic acid with feruloylesterases ${ }^{(437,438)}$, and phytic acid with exogenous or endogenous phytases (i.e. through adding degrading fungal and microbial enzymes, genetic engineering to over-express phytase activity and food processes to activate endogenous phytases $^{(217)}$ ) have been considered with the objective of increasing the bioactive potential of whole-grain cereal foods, and in the end their nutritional value.

Practically, this could be also partly achieved by using traditional and natural processes such as germination, soaking and/or fermentation in a highly hydrated medium. The fermentation of whole-grain cereals such as wheat, maize, rice, sorghum and millet, either germinated or not, often in combination with leguminous seeds (for example, soyabean and chickpea), is widespread in developed countries and the Orient for whole-grain cereal-based beverages, gruels and porridges (for example, koko, doro, ogi, akasa, tuo zaafi and togwa in Africa; idli in India; shoyu in the Orient; chicha in South America; or kishk in Arabian countries). It increases the nutritional density of the products, protects against diarrhoea, is easy to apply, allows a good preservation of the products (useful, for example, for long displacements), may improve sensory quality and is inexpensive ${ }^{(43-441)}$. Before fermentation, whole-grain cereals are generally soaked, germinated, dried and coarsely ground with a grinding stone ${ }^{(440)}$. Fermentation, by activating enzymes, can release bound bioactive compounds, synthetise new bioactive compounds, degrade antinutrients and increase protein and starch digestibility ${ }^{(439)}$. This is accompanied by numerous potential positive health effects as recently reviewed, for example, improved gut health or reduction of the rate of starch degradation ${ }^{(442)}$. Thus, germination and fermentation have been used for whole-grain wheat, rye, maize, sorghum and millet in order to decrease the tannin and phytic acid contents, as both compounds impair mineral bioavailability - leading to Fedeficiency anaemia in developing countries - and also in order to increase the protein/gluten and starch digestibility and the concentration of free amino acids by enhanced proteolytic and $\alpha$-amylolytic activities $(177,178,180,443-449)$. Sourdough pre-fermentation (incubation for $24 \mathrm{~h}$ at $30^{\circ} \mathrm{C}$ with lactic acid bacteria) for whole-wheat flour degrades about $60-70 \%$ of the phytic acid in bread dough (compared with the initial flour content) in $4 \mathrm{~h}$, so increasing $\mathrm{Mg}$ bioaccessibility in vitro $^{(220,450)}$ and in vivo in rats ${ }^{(451)}$. In another study, the type of starter for sourdough fermentation and the type of raw material (native $v$. malted or germinated rye) was shown to influence the content in bioactive compounds of the resulting wholemeal rye flour. The combination of germination and fermentation increased the levels of folates (7-fold), free phenolic acids (10-fold), total phenolic compounds (4-fold), lignans (3-fold) and alkylresorcinols, but, to a lesser extent $(<1 \cdot 5$-fold $)$ the metabolic activities of microbes together with the breakdown and hydrolysis of some cereal cell walls were involved in this effect $^{(452)}$. Conversely, a $4 \mathrm{~h}$ sourdough fermentation of whole-wheat flour leads to losses of alkylresorcinol ${ }^{(453)}$. The fermentation of rye bran also enhances the free ferulic acid and the solubilisation of pentosans through xylanase activation $^{(454)}$. Recently, an increased level of free ferulic acid (about a 2-fold increase) has been reported within whole-wheat dough pizza upon 18 and $48 \mathrm{~h}$ of fermentation $^{(455)}$, as well as an increase in pentosan solubilisation and prolamin hydrolysis in germinated rye sourdough ${ }^{(446)}$. This could have practical nutritional implications as discussed earlier with free ferulic acid, and also since the soluble fraction of arabinoxylans has been shown to reduce the glycaemic response in either healthy subjects ${ }^{(411)}$ or in those with impaired glucose tolerance ${ }^{(456)}$. On the other hand, prolamin proteins are known to trigger coeliac disease (autoimmune disorder due to gluten intolerance) and their intensive pre-hydrolysis during germination and fermentation might render cereal products from these technologies coeliac-safe ${ }^{(446)}$. Lastly, fermentation of whole-grain cereals has been reported in several studies to increase the content of available methionine and B vitamins, such as thiamin, riboflavin, niacin, folates and pantothenic acid, through the action of micro-organisms ${ }^{(439)}$. Despite all these convincing results, the health benefits of hydrolysis and/or the release of free bioactive compounds from whole-grain cereal products through germination and/or fermentation have not been sufficiently explored in human subjects. 
The addition of a pre-fermentation step before processing other cereal products, such as those usually widely consumed in our Western societies (for example, breakfast cereals or crackers), should also be studied more. A recent study showed that adding a pre-fermentation step while omitting steam cooking before wheat flake processing preserved a satisfactorily nutritional quality by improving the management of the feeling of hunger in the morning and by moderately improving insulin economy, which could be of interest for type 2 diabetic subjects ${ }^{(457)}$.

Whole-grain and wholemeal breads are generally made of flours with an extraction rate of 85-90\% (type 80 flours). Baking these flours does not sufficiently degrade phytic acid or hydrate the fibre fraction. These flours also do not generally contain the germ fraction, leading to a loss of $\mathrm{B}$ vitamins. One alternative would be to add 20 to $30 \%$ wholegrain flour (with an extraction rate of $100 \%$ ) to white wheat flour $^{(441)}$. The whole-grain flour could be pre-fermented in a strongly hydrated medium with leaven, and then reincorporated into white flour for baking to avoid hydration competing with gluten and fibre. This adds the germ fraction together with a significant increase in bioactive compounds while partially degrading phytic acid ${ }^{(441)}$. Sourdough whole-grain barley and wheat breads also reduce the glycaemic response in healthy subjects through delayed gastric emptying and possibly through a higher content of RS, thus prolonging satiety with potential benefits in weight control $^{(458,459)}$.

Reinforcing the food structure cohesiveness in processed cereal products. As preserving intact the botanical structure in whole-grain cereal products and favouring compactness of processed cereal products such as pasta reduces the glycaemic and insulinaemic responses and increases satiety, both of which are useful in the management of type 2 diabetes and weight regulation, processed cereal products with greater cohesiveness need to be identified. This can be achieved artificially by creating protein and/or fibrous networks in the food matrix to hinder enzyme accessibility to its substrate within the small intestine ${ }^{(460)}$, by using intact cereal kernels with a natural fibrous network ${ }^{(51,54)}$, and/or by altering kneading intensities and proving time during baking to obtain breads with a more dense crumb texture ${ }^{(461)}$. Some have also tried, with relative success, to increase the thickness of breakfast cereal flakes to reduce their glycaemic and insulinaemic indices in healthy subjects ${ }^{(462)}$. The more frequent use of more or less intact whole-grain cereal kernels in food recipes seems the most promising, easiest and cheapest way to explore by technologists.

Isolating the aleurone layer from the wheat bran fraction. Since most of the bioactive compounds are in the aleurone layer of the bran ${ }^{(463)}$ and since the pericarp (especially the outer fraction composed of cellulose, penstosans and lignins is poorly digestible) may contain contaminants (pesticides, mycotoxins and heavy metals), antinutrient compounds, irritants for the digestive epithelium (for example, lignins and insoluble fibre) and may limit the bioavailability of bioactive compounds, different processes for isolating the aleurone layer from wheat bran have been investigated ${ }^{(464-466)}$, with the objective of reincorporating it in cereal food recipes. This appears to be a new way of enhancing the nutrition value of cereal products ${ }^{(464,466)}$. The aleurone layer represents approximately 6-9\% of the whole-grain wheat (Fig. 1). Some researchers have studied the nutritional quality of aleurone flour, and shown that the aleurone layer is a rich source of bioavailable folate in humans ${ }^{(467)}$, that it lowers plasma homocysteine ${ }^{(468)}$, increases SCFA production ${ }^{(469)}$, reduces colon adenoma in azoxymethane-treated rats ${ }^{(470)}$, and that it is more digestible $(+17 \%)$ and fermentable $(+30 \%)$ than wheat bran, so yielding more butyrate ${ }^{(471)}$. It also has a higher antioxidant activity than wheat bran (1.5-fold) and whole-grain wheat (2-fold) in vitro ${ }^{(132,464)}$. However, isolating the aleurone layer from the bran fraction means losing the health benefits of lignins (mainly in the outer pericarp and testa layers of the bran fraction), which seem to be significant and remain largely unknown (see above). The long-term benefit of consuming bran and aleurone fractions on several physiological parameters and major health problems is therefore an important issue that should be explored in order to assess the real nutritional value of lignins and decide whether the few negative physiological effects generally associated with lignins are outweighed by their positive effects. The issue is close to that of phytic acid, which also has both negative and positive physiological effects. However, the issue of preserving the lignin would be the most meaningful in the case of organic whole-grain cereals which should not contain pesticides in their outer pericarp.

\section{Conclusions}

The nutritional quality of cereal products may therefore be improved by agricultural conditions, genetics and technological processes. Organic agriculture, genetics, the use of a pre-fermentation step and of a more or less intact grain structure are probably the most promising ways to preserve and enhance the nutritional density of whole-grain foods. Sourdough pre-fermentation could also be used for other whole-grain cereal foods such as breakfast cereals. The first parameter described in Fig. 5 is the milling process, and the best way to preserve a high nutritional density in bioactive compounds is to use flours with high extraction rates. It must be remembered that whole-grain wheat, wheat bran and wheat germ contain, respectively, at least 15,52 and at least $24 \%$ bioactive compounds and dietary fibre (Table 1 ). Removing the bran fraction during milling and using it to feed animals is therefore an issue to consider more seriously.

\section{General conclusions}

\section{The importance of preserving bran and germ fractions}

The bioactive compounds in whole-grain cereals are unevenly distributed (Fig. 1). Some (mainly soluble fibre, $\mathrm{Se}$, some B vitamins, carotenoids and flavonoids) are present in significant quantities in the endosperm, but most are in the bran (especially the aleurone layer) and germ fractions. This fact alone shows the importance of preserving these fractions in cereal products, at least in the most currently consumed forms of breads and breakfast cereals, and to a lesser extent pasta, crackers and biscuits. 
Some products consumed on special occasions (i.e. generally not at breakfast, lunch or dinner), such as cakes, pastries and viennoiseries, use very refined flours (extraction rate of $70-82 \%$ ), and it is probably not meaningful to use less refined flours. To preserve the bran and germ fractions means either reincorporating fractions later in the recipe or using the whole-grain cereal so as to maintain its botanical structure relatively intact during processing. However, reincorporation of the bran and germ fractions implies destroying the botanical structure with the loss of its health benefits (for example, increased satiety or RS content), unless technological processes can yield a cereal product with an artificial compact food structure as for pasta ${ }^{(472)}$ or breads with decreased loaf volume ${ }^{(461)}$.

\section{The concept of the 'whole-grain package'}

The content of individual bioactive compounds in whole grain often seems too low for them to have any significant or lasting physiological effects. It is becoming more and more evident that the synergetic action of several bioactive compounds contributes to health protection and/or the maintenance of one physiological function, not just one compound. Fig. 1 and Table 4 illustrate this concept of the 'whole-grain package': thus, obesity/body-weight regulation, CVD, type 2 diabetes, cancers, gut, mental/nervous system and skeleton health may be potentially protected by at least, respectively, ten, thirty-four, seventeen, thirty-two, ten, twenty-six and sixteen different bioactive compounds and/or groups of compounds (i.e. oligosaccharides, tocols, phenolic acids, flavonoids, saponins, inositols, $\gamma$-oryzanol, lignans and alkylresorcinols). Because of their many protective bioactive compounds (at least twenty-six), whole-grain cereals are particularly suitable for protecting the body from CVD, cancers and mental/nervous system disorders. The long-term protection against mental or nervous system disorders by consuming whole-grain cereal products therefore deserves to be studied in human subjects, notably because depression ranks among the major causes of mortality and disability with an overall prevalence of $5-8 \%{ }^{(274)}$. It is also remarkable that at least thirty compounds and/or groups of compounds may participate in antioxidant protection through different mechanisms (Tables 3 and 4), which approximately corresponds to a total of at least $3.9,13.4$ and $6.3 \%$ of the whole-grain wheat, wheat bran and germ fractions (Tables 1 and 2). As most age-related and chronic diseases are associated with increased oxidative stress, the regular consumption of whole-grain cereal products should benefit all of us, but particularly the elderly.

\section{The importance of pesticides and mycotoxins}

Since whole-grain cereals include by definition the outer parts of the grain, they may contain pesticides and mycotoxins (for example, zearalenone and deoxynivalenol in wheat or fumonisin in maize). Their presence should not decrease the benefits of bioactive compounds also mainly contained in the outer layers. For example, there may be a relationship between the consumption of fumonisincontaminated maize in some regions of the world (for example, China and South Africa) and the occurrence of oesophageal cancers $^{(473,474)}$. However, more generally, the consequences of long-term consumption of high quantities of mycotoxin-contaminated cereal grains for human health (i.e. toxicological effects) are not well known. The link between some cancers and exposure to pesticides has been well established, particularly among farmers ${ }^{(475)}$. It is therefore particularly relevant that recommendations for the consumption of more whole-grain cereal products should be accompanied by the production of less contaminated cereals, such as those from organic agriculture devoid of pesticides.

\section{Perspectives}

It is surprising to note that, although numerous epidemiological surveys have shown a significant and positive association between whole-grain cereal consumption and the prevention of several chronic diseases, fewer studies have been performed on the mechanisms involved. For example, to my knowledge, no more than eleven studies have examined the antioxidant hypothesis by postprandial or intervention studies in human subjects to investigate the antioxidant effect of whole-grain cereals, bran or germ ${ }^{(136)}$, with only a recent postprandial study on human subjects consuming wheat bran ${ }^{(146)}$. Therefore, there is a real gap between observational studies and the elucidation of the mechanisms involved. The mechanisms are certainly complex, as has been seen. But more data are needed on the mechanisms involved so as to prepare strong, convincing arguments for an increased consumption of whole-grain cereal products by the public, to better inform health professionals about their health benefits, to favour their marketing by the food industry and to develop new health claims in the near future.

\section{Acknowledgements}

I thank Dr Christian Rémésy for his constructive criticism of the manuscript and Professor Inger Björck (Department of Applied Nutrition and Food Chemistry, Chemical Centre, Lund University, Sweden) for allowing me to use her original diagram (from the HealthGrain Project, European Community's Sixth Framework Programme, FOOD-CT2005-514008, 2005-2010) that I have adapted for Fig. 2 of the paper (see original diagram in the brochure 'Progress in HEALTHGRAIN 2008' at http://www.healthgrain.org/pub/). The English text of the manuscript has been checked by Dr Owen Parkes.

There are no conflicts of interest and the present review received no specific grant from any funding agency in the public, commercial or not-for-profit sectors.

Colour versions of Figs. 1, 2 and 4 can be seen in the online version of the paper.

\section{References}

1. Koh-Banerjee P \& Rimm EB (2003) Whole grain consumption and weight gain: a review of the epidemiological evidence, potential mechanisms and opportunities for future research. Proc Nutr Soc 62, 25-29.

2. van de Vijver LPL, van den Bosch LMC, van den Brandt PA, et al. (2009) Whole-grain consumption, dietary fibre 
intake and body mass index in the Netherlands cohort study. Eur J Clin Nutr 63, 31-38.

3. Esmaillzadeh A, Mirmiran P \& Azizi F (2005) Wholegrain consumption and the metabolic syndrome: a favorable association in Tehranian adults. Eur J Clin Nutr 59, 353-362.

4. Sahyoun NR, Jacques PF, Zhang XL, et al. (2006) Wholegrain intake is inversely associated with the metabolic syndrome and mortality in older adults. Am J Clin Nutr 83, 124-131.

5. de Munter JS, Hu FB, Spiegelman D, et al. (2007) Whole grain, bran, and germ intake and risk of type 2 diabetes: a prospective cohort study and systematic review. PLoS Med 4, e261.

6. Murtaugh MA, Jacobs DR, Jacob B, et al. (2007) Epidemiological support for the protection of whole grains against diabetes. Proc Nutr Soc 62, 143-149.

7. Mellen PB, Walsh TF \& Herrington DM (2008) Whole grain intake and cardiovascular disease: a meta-analysis. Nutr Metab Cardiovasc Dis 18, 283-290.

8. Chan JM, Wang F \& Holly EA (2007) Whole grains and risk of pancreatic cancer in a large population-based casecontrol study in the San Francisco Bay area, California. Am J Epidemiol 166, 1174-1185.

9. Chatenoud L, Tavani A, La Vecchia C, et al. (1998) Whole grain food intake and cancer risk. Int J Cancer 77, 24-28.

10. Jacobs DR Jr, Marquart L, Slavin J, et al. (1998) Whole-grain intake and cancer: an expanded review and meta-analysis. Nutr Cancer 30, 85-96.

11. Larsson SC, Giovannucci E, Bergkvist L, et al. (2005) Whole grain consumption and risk of colorectal cancer: a population-based cohort of 60000 women. Br J Cancer 92, $1803-1807$.

12. Schatzkin A, Park Y, Leitzmann MF, et al. (2008) Prospective study of dietary fiber, whole grain foods, and small intestinal cancer. Gastroenterology 135, 1163-1167.

13. Jacobs DR Jr, Andersen LF \& Blomhoff R (2007) Wholegrain consumption is associated with a reduced risk of noncardiovascular, noncancer death attributed to inflammatory diseases in the Iowa Women's Health Study. Am J Clin Nutr 85, 1606-1614.

14. Adom KK, Sorrells ME \& Liu RH (2003) Phytochemical profiles and antioxidant activity of wheat varieties. J Agric Food Chem 51, 7825-7834.

15. Jacobs DR, Meyer HE \& Solvoll K (2001) Reduced mortality among whole grain bread eaters in men and women in the Norwegian County Study. Eur J Clin Nutr 55, 137-143.

16. Chatenoud L, La Vecchia C, Franceschi S, et al. (1999) Refined-cereal intake and risk of selected cancers in Italy. Am J Clin Nutr 70, 1107-1110.

17. Jensen MK, Koh-Banerjee P, Franz M, et al. (2006) Whole grains, bran, and germ in relation to homocysteine and markers of glycemic control, lipids, and inflammation. Am J Clin Nutr 83, 275-283.

18. Liu RH (2007) Whole grain phytochemicals and health. J Cereal Sci 46, 207-219.

19. Truswell AS (2002) Cereal grains and coronary heart disease. Eur J Clin Nutr 56, 1-14.

20. AACC International (1999) Definition of whole grain. http://www.aaccnet.org/definitions/wholegrain.asp (accessed January 2010).

21. European Food Information Council (EUFIC) (2009) Whole grain fact sheet. http://www.eufic.org/article/en/ page/BARCHIVE/expid/Whole-grain-Fact-Sheet/ (accessed January 2010).

22. Food and Drug Adminstration (2006) Draft Guidance for Industry and FDA Staff: Whole Grain Label Statements.
http://www.fda.gov/Food/GuidanceCompliance RegulatoryInformation/GuidanceDocuments/Food LabelingNutrition/UCM059088 (accessed January 2010).

23. AACC International (2006) AACC International comments on Part III of the Draft Guidance on Whole Grain Label Statements. http://www.aaccnet.org/definitions/ pdfs/AACCIntlWholeGrainComments.pdf (accessed January 2010).

24. Jones JM (2008) Whole grains - issues and deliberations from the Whole Grain Task Force. Cereal Foods World $\mathbf{5 3}$ $260-264$.

25. Srivastava AK, Sudha ML, Baskaran V, et al. (2007) Studies on heat stabilized wheat germ and its influence on rheological characteristics of dough. Eur Food Res Technol 224, 365-372.

26. Food and Drug Administration (1999) Health Claim Notification for Whole Grain Foods. http://www.fda.gov/ Food/LabelingNutrition/LabelClaims/FDAModernization ActFDAMAClaims/UCM073639.htm (accessed January 2010).

27. Jacobs DR Jr, Meyer KA, Kushi LH, et al. (1998) Wholegrain intake may reduce the risk of ischemic heart disease death in postmenopausal women: the Iowa Women's Health Study. Am J Clin Nutr 68, 248-257.

28. Liu SM, Manson JE, Stampfer MJ, et al. (2000) Whole grain consumption and risk of ischemic stroke in women a prospective study. JAMA 284, 1534-1540.

29. Thane CW, Jones AR, Stephen AM, et al. (2005) Wholegrain intake of British young people aged 4-18 years. Br J Nutr 94, 825-831.

30. Thane CW, Stephen AM \& Jebb SA (2009) Whole grains and adiposity: little association among British adults. Eur J Clin Nutr 63, 229-237.

31. Cleveland LE, Moshfegh AJ, Albertson AM, et al. (2000) Dietary intake of whole grains. J Am Coll Nutr 19, 331S-338S.

32. Lang R \& Jebb SA (2003) Who consumes whole grains, and how much? Proc Nutr Soc 62, 123-127.

33. Welsh S, Shaw A \& Davis C (1994) Achieving dietary recommendations - whole-grain foods in the food guide pyramid. Crit Rev Food Sci Nutr 34, 441-451.

34. Albertson AM \& Tobelmann RC (1995) Consumption of grain and whole-grain foods by an American population during the years 1990 to 1992. JAm Diet Assoc 95, 703-704.

35. National Council on Nutrition and Physical Activity and the Institute for Nutrition Research (1998) Development of the Norwegian Diet. Oslo: University of Oslo.

36. Prättälä R, Helasoja V \& Mykkänen H (2007) The consumption of rye bread and white bread as dimensions of health lifestyles in Finland. Public Health Nutr 4, 813-819.

37. Adams JF \& Engstrom A (2000) Helping consumers achieve recommended intakes of whole grain foods. $J$ Am Coll Nutr 19, 339S-344S.

38. Jenkins DJ, Wesson V, Wolever TM, et al. (1988) Wholemeal versus wholegrain breads: proportion of whole or cracked grain and the glycaemic response. BMJ 297, 958-960.

39. Jacobs DR Jr, Pereira MA, Stumpf K, et al. (2002) Whole grain food intake elevates serum enterolactone. $\mathrm{Br} \mathrm{J} \mathrm{Nutr}$ 88, $111-116$

40. Lutsey PL, Jacobs DR, Kori S, et al. (2007) Whole grain intake and its cross-sectional association with obesity, insulin resistance, inflammation, diabetes and subclinical CVD: The MESA Study. Br J Nutr 98, 397-405.

41. McKeown NM, Meigs JB, Liu S, et al. (2002) Whole-grain intake is favorably associated with metabolic risk factors for type 2 diabetes and cardiovascular disease in the 
Framingham Offspring Study. Am J Clin Nutr 76, 390-398.

42. Newby P, Maras J, Bakun P, et al. (2007) Intake of whole grains, refined grains, and cereal fiber measured with 7-d diet records and associations with risk factors for chronic disease. Am J Clin Nutr 86, 1745-1753.

43. Vanharanta M, Voutilainen S, Lakka TA, et al. (1999) Risk of acute coronary events according to serum concentrations of enterolactone: a prospective population-based case-control study. Lancet 354, 2112-2115.

44. Levi F, Pasche C, Lucchini F, et al. (2000) Refined and whole grain cereals and the risk of oral, oesophageal and laryngeal cancer. Eur J Clin Nutr 54, 487-489.

45. Slavin JL (2000) Mechanisms for the impact of whole grain foods on cancer risk. J Am Coll Nutr 19, 300S-307S.

46. Slavin JL, Martini MC, Jacobs DR Jr, et al. (1999) Plausible mechanisms for the protectiveness of whole grains. Am J Clin Nutr 70, 459S-463S.

47. Haber GB, Heaton KW, Murphy D, et al. (1977) Depletion and disruption of dietary fibre. Effects on satiety, plasmaglucose, and serum-insulin. Lancet ii, 679-682.

48. Read NW, Welch IM, Austen CJ, et al. (1986) Swallowing food without chewing; a simple way to reduce postprandial glycaemia. Br J Nutr 55, 43-47.

49. Fardet A, Leenhardt F, Lioger D, et al. (2006) Parameters controlling the glycaemic response to breads. Nutr Res Rev 19, $18-25$.

50. Granfeldt Y, Hagander B \& Bjorck I (1995) Metabolic responses to starch in oat and wheat products. On the importance of food structure, incomplete gelatinization or presence of viscous dietary fibre. Eur J Clin Nutr 49, 189-199.

51. Liljeberg H, Granfeldt Y \& Bjorck I (1992) Metabolic responses to starch in bread containing intact kernels versus milled flour. Eur J Clin Nutr 46, 561-575.

52. Nilsson AC, Ostman EM, Granfeldt Y, et al. (2008) Effect of cereal test breakfasts differing in glycemic index and content of indigestible carbohydrates on daylong glucose tolerance in healthy subjects. Am J Clin Nutr 87, 645-654.

53. American Association of Cereal Chemists (2001) The definition of dietary fiber. Cereal Foods World 46, 112-129.

54. Nilsson AC, Ostman EM, Holst JJ, et al. (2008) Including indigestible carbohydrates in the evening meal of healthy subjects improves glucose tolerance, lowers inflammatory markers, and increases satiety after a subsequent standardized breakfast. J Nutr 138, 732-739.

55. Topping D (2007) Cereal complex carbohydrates and their contribution to human health. J Cereal Sci 46, 220-229.

56. Wood PJ (2007) Cereal $\beta$-glucans in diet and health. $J$ Cereal Sci 46, 230-238.

57. Koh-Banerjee P, Franz M, Sampson L, et al. (2004) Changes in whole-grain, bran, and cereal fiber consumption in relation to 8-y weight gain among men. Am J Clin Nutr 80, 1237-1245.

58. Slavin JL (2005) Dietary fiber and body weight. Nutrition 21, 411-418.

59. Jenkins AL, Jenkins DJ, Zdravkovic U, et al. (2002) Depression of the glycemic index by high levels of $\beta$ glucan fiber in two functional foods tested in type 2 diabetes. Eur J Clin Nutr 56, 622-628.

60. Zhang JX, Hallmans G, Andersson H, et al. (1992) Effect of oat bran on plasma cholesterol and bile acid excretion in nine subjects with ileostomies. Am J Clin Nutr 56, 99-105.

61. Lia A, Hallmans G, Sandberg AS, et al. (1995) Oat $\beta$ glucan increases bile acid excretion and a fiber-rich barley fraction increases cholesterol excretion in ileostomy subjects. Am J Clin Nutr 62, 1245-1251.
62. Scheppach W, Bartram HP \& Richter F (1995) Role of short-chain fatty acids in the prevention of colorectal cancer. Eur J Cancer 31, 1077-1080.

63. Slavin J (2003) Why whole grains are protective: biological mechanisms. Proc Nutr Soc 62, 129-134.

64. Costabile A, Klinder A, Fava F, et al. (2008) Whole-grain wheat breakfast cereal has a prebiotic effect on the human gut microbiota: a double-blind, placebo-controlled, crossover study. Br J Nutr 99, 110-120.

65. Brouns F, Kettlitz B \& Arrigoni E (2002) Resistant starch and 'the butyrate revolution'. Trends Food Sci Technol 13, 251-261.

66. Boffa LC, Lupton JR, Mariani MR, et al. (1992) Modulation of colonic epithelial cell proliferation, histone acetylation, and luminal short chain fatty acids by variation of dietary fiber (wheat bran) in rats. Cancer Res 52, 5906-5912.

67. Higgins J, Higbee D, Donahoo W, et al. (2004) Resistant starch consumption promotes lipid oxidation. Nutr Metab 1, 8.

68. McIntosh GH, Noakes M, Royle PJ, et al. (2003) Wholegrain rye and wheat foods and markers of bowel health in overweight middle-aged men. Am J Clin Nutr 77, 967-974.

69. Ferguson LR \& Harris PJ (1999) Protection against cancer by wheat bran: role of dietary fibre and phytochemicals. Eur J Cancer Prev 8, 17-25.

70. Lopez HW, Coudray C, Bellanger J, et al. (2000) Resistant starch improves mineral assimilation in rats adapted to a wheat bran diet. Nutr Res 20, 141-155.

71. Lopez HW, Levrat-Verny MA, Coudray C, et al. (2001) Class 2 resistant starches lower plasma and liver lipids and improve mineral retention in rats. J Nutr 131, 1283-1289.

72. Coudray C, Bellanger J, Castiglia-Delavaud C, et al. (1997) Effect of soluble or partly soluble dietary fibres supplementation on absorption and balance of calcium, magnesium, iron and zinc in healthy young men. Eur $J$ Clin Nutr 51, 375-380.

73. Lopez HW, Coudray C, Levrat-Verny MA, et al. (2000) Fructooligosaccharides enhance mineral apparent absorption and counteract the deleterious effects of phytic acid on mineral homeostasis in rats. J Nutr Biochem 11, 500-508.

74. Reddy B, Hamid R \& Rao C (1997) Effect of dietary oligofructose and inulin on colonic preneoplastic aberrant crypt foci inhibition. Carcinogenesis 18, 1371-1374.

75. McIntyre A, Gibson PR \& Young GP (1993) Butyrate production from dietary fiber and protection against large bowel cancer in a rat model. Gut 34, 386-391.

76. Heerdt BG, Houston MA, Anthony GM, et al. (1999) Initiation of growth arrest and apoptosis of MCF-7 mammary carcinoma cells by tributyrin, a triglyceride analogue of the short-chain fatty acid butyrate, is associated with mitochondrial activity. Cancer Res 59, 1584-1591.

77. Ellerhorst J, Nguyen T, Cooper DNW, et al. (1999) Induction of differentiation and apoptosis in the prostate cancer cell line LNCaP by sodium butyrate and galectin- 1 . Int J Oncol 14, 225-232.

78. Bird AR, Vuaran MS, King RA, et al. (2008) Wholegrain foods made from a novel high-amylose barley variety (Himalaya 292) improve indices of bowel health in human subjects. Br J Nutr 99, 1032-1040.

79. Liljeberg H \& Bjorck I (1994) Bioavailability of starch in bread products. Postprandial glucose and insulin responses in healthy subjects and in vitro resistant starch content. Eur J Clin Nutr 48, 151-163.

80. Hara H, Haga S, Aoyama Y, et al. (1999) Short-chain fatty acids suppress cholesterol synthesis in rat liver and intestine. J Nutr 129, 942-948. 
81. Jenkins DJA, Wolever TMS, Nineham R, et al. (1980) Improved glucose tolerance four hours after taking guar with glucose. Diabetologia 19, 21-24.

82. Jenkins D, Wolever T, Taylor R, et al. (1982) Slow release dietary carbohydrate improves second meal tolerance. Am J Clin Nutr 35, 1339-1346.

83. Nilsson A, Granfeldt Y, Ostman E, et al. (2006) Effects of GI and content of indigestible carbohydrates of cerealbased evening meals on glucose tolerance at a subsequent standardised breakfast. Eur J Clin Nutr 60, 1092-1099.

84. Cherbut C (2003) Motor effects of short-chain fatty acids and lactate in the gastrointestinal tract. Proc Nutr Soc 62 , 95-99.

85. Wolever T, Spadafora P \& Eshuis H (1991) Interaction between colonic acetate and propionate in humans. Am J Clin Nutr 53, 681-687.

86. Homko CJ, Cheung P \& Boden G (2003) Effects of free fatty acids on glucose uptake and utilization in healthy women. Diabetes 52, 487-491.

87. Anderson JW \& Bridges SR (1984) Short-chain fatty acid fermentation products of plant fiber affect glucose metabolism of isolated rat hepatocytes. Proc Soc Exp Biol Med 177, 372-376.

88. Liu RH (2004) Potential synergy of phytochemicals in cancer prevention: mechanism of action. J Nutr 134, 3479S-3485S.

89. Reddy BS, Hirose Y, Cohen LA, et al. (2000) Preventive potential of wheat bran fractions against experimental colon carcinogenesis: implications for human colon cancer prevention. Cancer Res 60, 4792-4797.

90. Sang SM, Ju JY, Lambert JD, et al. (2006) Wheat bran oil and its fractions inhibit human colon cancer cell growth and intestinal tumorigenesis in Apc(min/+) mice. J Agric Food Chem 54, 9792-9797.

91. Bartsch H \& Nair J (2006) Chronic inflammation and oxidative stress in the genesis and perpetuation of cancer: role of lipid peroxidation, DNA damage, and repair. Langenbecks Arch Surg 391, 499-510.

92. Graf E \& Eaton JW (1985) Dietary suppression of colonic cancer; fiber or phytate? Cancer 56, 717-718.

93. Kohlmeier L, Simonsen N \& Mottus K (1995) Dietary modifiers of carcinogenesis. Environ Health Perspect 103, Suppl. 8, 177-184.

94. Nesaretnam K, Yew WW \& Wahid MB (2007) Tocotrienols and cancer: beyond antioxidant activity. Eur J Lipid Sci Technol 109, 445-452.

95. Shamsuddin AM (2002) Anti-cancer function of phytic acid. Int J Food Sci Technol 37, 769-782.

96. Adlercreutz H (2002) Phyto-oestrogens and cancer. Lancet Oncol 3, 364-373.

97. Adlercreutz H, Mousavi Y, Clark J, et al. (1992) Dietary phytoestrogens and cancer: in vitro and in vivo studies. J Steroid Biochem Molec Biol 41, 331-337.

98. Markaverich BM, Webb B, Densmore CL, et al. (1995) Effects of coumestrol on estrogen receptor function and uterine growth in ovariectomized rats. Environ Health Perspect 103, 574-581.

99. Reddy BS (1999) Prevention of colon carcinogenesis by components of dietary fiber. Anticancer Res 19, $3681-3683$.

100. Ullah A \& Shamsuddin AM (1990) Dose-dependent inhibition of large intestinal cancer by inositol hexaphosphate in F344 rats. Carcinogenesis 11, 2219-2222.

101. Vucenik I, Yang G \& Shamsuddin AM (1997) Comparison of pure inositol hexaphosphate and high-bran diet in the prevention of DMBA-induced rat mammary carcinogenesis. Nutr Cancer 28, 7-13.
102. Hollman P \& Katan M (1997) Absorption, metabolism and health effects of dietary flavonoids in man. Biomed Pharmacother 51, 305-310.

103. Edenharder R, Rauscher R \& Platt KL (1997) The inhibition by flavonoids of 2-amino-3-methylimidazo[4,5f] quinoline metabolic activation to a mutagen: a structureactivity relationship study. Mutat Res 379, 21-32.

104. Barone E, Calabrese V \& Mancuso C (2009) Ferulic acid and its therapeutic potential as a hormetin for age-related diseases. Biogerontology 10, 97-108.

105. Kawabata K, Yamamoto T, Hara A, et al. (2000) Modifying effects of ferulic acid on azoxymethaneinduced colon carcinogenesis in F344 rats. Cancer Lett 157, 15-21.

106. Alabaster O, Tang ZC \& Shivapurkar N (1996) Dietary fiber and the chemopreventive modelation of colon carcinogenesis. Mutat Res 350, 185-197.

107. Eastwood MA \& Girdwood RH (1968) Lignin: a bile-salt sequestrating agent. Lancet ii, 1170-1172.

108. Eastwood MA \& Hamilton D (1968) Studies on the adsorption of bile salts to non-absorbed components of diet. Biochim Biophys Acta 152, 165-173.

109. Labaj J, Slamenova D, Lazarova M, et al. (2004) Ligninstimulated reduction of oxidative DNA lesions in testicular cells and lymphocytes of Sprague-Dawley rats in vitro and ex vivo. Nutr Cancer 50, 198-205.

110. Wattenberg LW (1985) Chemoprevention of cancer. Cancer Res 45, 1-8.

111. Jablonska E, Gromadzinska J, Sobala W, et al. (2008) Joint effect of GPx1 polymorphism and selenium status on lung cancer risk. Eur J Cancer Suppl 6, 203.

112. Stavric B (1994) Antimutagens and anticarcinogens in foods. Food Chem Toxicol 32, 79-90.

113. Harris PJ \& Ferguson LR (1993) Dietary fiber - its composition and role in protection against colorectal cancer. Mutat Res 290, 97-110.

114. Harris PJ, Roberton AM, Watson ME, et al. (1993) The effects of soluble-fiber polysaccharides on the adsorption of a hydrophobic carcinogen to an insoluble dietary fiber. Nutr Cancer 19, 43-54.

115. Morita T, Tanabe H, Sugiyama K, et al. (2004) Dietary resistant starch alters the characteristics of colonic mucosa and exerts a protective effect on trinitrobenzene sulfonic acid-induced colitis in rats. Biosci Biotechnol Biochem $\mathbf{6 8}$, 2155-2164.

116. Toden S, Bird AR, Topping DL, et al. (2007) Dosedependent reduction of dietary protein-induced colonocyte DNA damage by resistant starch in rats correlates more highly with caecal butyrate than with other short chain fatty acids. Cancer Biol Ther 6, 253-258.

117. Story JA \& Kritchevsky D (1994) Denis Parsons Burkitt (1911-1993). J Nutr 124, 1551-1554.

118. Bauer-Marinovic M, Florian S, Muller-Schmehl K, et al (2006) Dietary resistant starch type 3 prevents tumor induction by 1,2-dimethylhydrazine and alters proliferation, apoptosis and dedifferentiation in rat colon. Carcinogenesis 27, 1849-1859.

119. Bingham SA (2007) Mechanisms and experimental and epidemiological evidence relating dietary fibre (non-starch polysaccharides) and starch to protection against large bowel cancer. Proc Nutr Soc 49, 153-171.

120. O'Keefe SJD, Kidd M, Espitalier-Noel G, et al. (1999) Rarity of colon cancer in Africans is associated with low animal product consumption, not fiber. Am J Gastroenterol 94, 1373-1380. 
121. Cho E, Willett WC, Colditz GA, et al. (2007) Dietary choline and betaine and the risk of distal colorectal adenoma in women. $J$ Natl Cancer Inst 99, 1224-1231.

122. Klaunig JE, Xu Y, Isenberg JS, et al. (1998) The role of oxidative stress in chemical carcinogenesis. Environ Health Perspect 106, 289-295.

123. Harris PJ \& Ferguson LR (1999) Dietary fibres may protect or enhance carcinogenesis. Mutat Res 443, 95-110.

124. Ford ES, Mokdad AH, Giles WH, et al. (2003) The metabolic syndrome and antioxidant concentrations: findings from the Third National Health and Nutrition Examination Survey. Diabetes 52, 2346-2352.

125. Higdon JV \& Frei B (2003) Obesity and oxidative stress a direct link to CVD? Arterioscler Thromb Vasc Biol 23, 365-367.

126. Keaney JF, Larson MG, Vasan RS, et al. (2002) Obesity as a source of systemic oxidative stress: clinical correlates of oxidative stress in the Framingham Study. Circulation 106, 467.

127. Evans JL, Goldfine ID, Maddux BA, et al. (2002) Oxidative stress and stress-activated signaling pathways: a unifying hypothesis of type 2 diabetes. Endocr Rev 23, 599-622.

128. Maiese K, Morhan SD \& Chong ZZ (2007) Oxidative stress biology and cell injury during type 1 and type 2 diabetes mellitus. Curr Neurovasc Res 4, 63-71.

129. Cai H \& Harrison DG (2000) Endothelial dysfunction in cardiovascular diseases: the role of oxidant stress. Circ Res 87, 840-844.

130. Castelao JE \& Gago-Dominguez M (2008) Risk factors for cardiovascular disease in women: relationship to lipid peroxidation and oxidative stress. Med Hypotheses 71, 39-44.

131. Martinez-Tome M, Murcia MA, Frega N, et al. (2004) Evaluation of antioxidant capacity of cereal brans. J Agric Food Chem 52, 4690-4699.

132. Miller HE, Rigelhof F, Marquart L, et al. (2000) Antioxidant content of whole grain breakfast cereals, fruits and vegetables. J Am Coll Nutr 19, 312S-319S.

133. Perez-Jimenez J \& Saura-Calixto F (2005) Literature data may underestimate the actual antioxidant capacity of cereals. J Agric Food Chem 53, 5036-5040.

134. Serpen A, Gökmen V, Pellegrini N, et al. (2008) Direct measurement of the total antioxidant capacity of cereal products. J Cereal Sci 48, 816-820.

135. Zielinski H \& Kozlowska H (2000) Antioxidant activity and total phenolics in selected cereal grains and their different morphological fractions. J Agric Food Chem $\mathbf{4 8}$, 2008-2016.

136. Fardet A, Rock E \& Rémésy C (2008) Is the in vitro antioxidant potential of whole-grain cereals and cereal products well reflected in vivo? J Cereal Sci 48, 258-276.

137. Andersson A, Tengblad S, Karlstrom B, et al. (2007) Whole-grain foods do not affect insulin sensitivity or markers of lipid peroxidation and inflammation in healthy, moderately overweight subjects. J Nutr 137, 1401-1407.

138. Beattie RK, Lee AM, Strain JJ, et al. (2003) Evaluation of the in vivo antioxidant activity of wheat bran in human subjects. Proc Nutr Soc 62, 17A.

139. Bruce B, Spiller GA, Klevay LM, et al. (2000) A diet high in whole and unrefined foods favorably alters lipids, antioxidant defenses, and colon function. $J$ Am Coll Nutr 19, 61-67.

140. Chen CYO, Milbury PE, Collins FW, et al. (2007) Avenanthramides are bioavailable and have antioxidant activity in humans after acute consumption of an enriched mixture from oats. J Nutr 137, 1375-1382.

141. Hamill LL, Keaveney EM, Price RK, et al. (2007) Assessment of antioxidant biomarkers in human plasma and urine after consumption of wheat bran and aleurone fractions. Proc Nutr Soc 66, 88A.

142. Jang Y, Lee JH, Kim OY, et al. (2001) Consumption of whole grain and legume powder reduces insulin demand, lipid peroxidation, and plasma homocysteine concentrations in patients with coronary artery disease: randomized controlled clinical trial. Arterioscler Thromb Vasc Biol 21, 2065-2071.

143. Kim JY, Kim JH, Lee DH, et al. (2008) Meal replacement with mixed rice is more effective than white rice in weight control, while improving antioxidant enzyme activity in obese women. Nutr Res 28, 66-71.

144. Lewis S, Bolton C \& Heaton K (1996) Lack of influence of intestinal transit on oxidative status in premenopausal women. Eur J Clin Nutr 50, 565-568.

145. Maki KC, Galant R, Samuel P, et al. (2007) Effects of consuming foods containing oat $\beta$-glucan on blood pressure, carbohydrate metabolism and biomarkers of oxidative stress in men and women with elevated blood pressure. Eur J Clin Nutr 61, 786-795.

146. Price RK, Welch RW, Lee-Manion AM, et al. (2008) Total phenolics and antioxidant potential in plasma and urine of humans after consumption of wheat bran. Cereal Chem $\mathbf{8 5}$, $152-157$.

147. Wang Q, Han PH, Zhang MW, et al. (2007) Supplementation of black rice pigment fraction improves antioxidant and anti-inflammatory status in patients with coronary heart disease. Asia Pac J Clin Nutr 16, 295-301.

148. Graf E, Empson KL \& Eaton JW (1987) Phytic acid. A natural antioxidant. J Biol Chem 262, 11647-11650.

149. Dizhbite T, Telysheva G, Jurkjane V, et al. (2004) Characterization of the radical scavenging activity of lignins - natural antioxidants. Bioresour Technol 95, 309-317.

150. Vitaglione P, Napolitano A \& Fogliano V (2008) Cereal dietary fibre: a natural functional ingredient to deliver phenolic compounds into the gut. Trends Food Sci Technol 19, 451-463.

151. Babbs CF (1990) Free radicals and the etiology of colon cancer. Free Radicic Biol Med 8, 191-200.

152. Adam A, Crespy V, Levrat-Verny MA, et al. (2002) The bioavailability of ferulic acid is governed primarily by the food matrix rather than its metabolism in intestine and liver in rats. $J$ Nutr 132, 1962-1968.

153. Mateo Anson N, van den Berg R, Havenaar R, et al. (2009) Bioavailability of ferulic acid is determined by its bioaccessibility. J Cereal Sci 49, 296-300.

154. Rondini L, Peyrat-Maillard MN, Marsset-Baglieri A, et al. (2004) Bound ferulic acid from bran is more bioavailable than the free compound in rat. J Agric Food Chem 52, 4338-4343.

155. Pellegrini N, Serafini M, Salvatore S, et al. (2006) Total antioxidant capacity of spices, dried fruits, nuts, pulses, cereals and sweets consumed in Italy assessed by three different in vitro assays. Mol Nutr Food Res 50, 1030-1038.

156. McCarty MF (2005) Magnesium may mediate the favorable impact of whole grains on insulin sensitivity by acting as a mild calcium antagonist. Med Hypotheses 64, 619-627.

157. Durlach J \& Collery P (1984) Magnesium and potassium in diabetes and carbohydrate metabolism. Review of the present status and recent results. Magnesium 3, 315-323. 
158. Paolisso G, Sgambato S, Gambardella A, et al. (1992) Daily magnesium supplements improve glucose handling in elderly subjects. Am J Clin Nutr 55, 1161-1167.

159. Paolisso G, Sgambato S, Pizza G, et al. (1989) Improved insulin response and action by chronic magnesium administration in aged NIDDM subjects. Diabetes Care 12, 265-269.

160. Pereira MA, Jacobs DR Jr, Pins JJ, et al. (2002) Effect of whole grains on insulin sensitivity in overweight hyperinsulinemic adults. Am J Clin Nutr 75, 848-855.

161. Balon T, Jasman A, Scott S, et al. (1994) Dietary magnesium prevents fructose-induced insulin insensitivity in rats. Hypertension 23, 1036-1039.

162. Balon TW, Gu JL, Tokuyama Y, et al. (1995) Magnesium supplementation reduces development of diabetes in a rat model of spontaneous NIDDM. Am J Physiol Endocrinol Metab 269, E745-E752.

163. Gould MK \& Chaudry IH (1970) The action of insulin on glucose uptake by isolated rat soleus muscle. 1. Effects of cations. Biochim Biophys Acta 215, 249-257.

164. Weglicki WB, Mak IT, Kramer JH, et al. (1996) Role of free radicals and substance $\mathrm{P}$ in magnesium deficiency. Cardiovasc Res 31, 677-682.

165. Ceriello A, Bortolotti N, Crescentini A, et al. (1998) Antioxidant defences are reduced during the oral glucose tolerance test in normal and non-insulin-dependent diabetic subjects. Eur J Clin Invest 28, 329-333.

166. Pereira EC, Ferderbar S, Bertolami MC, et al. (2008) Biomarkers of oxidative stress and endothelial dysfunction in glucose intolerance and diabetes mellitus. Clin Biochem 41, 1454-1460.

167. Iseri LT \& French JH (1984) Magnesium - Nature's physiologic calcium blocker. Am Heart J 108, 188-193.

168. Resnick LM (1992) Cellular calcium and magnesium metabolism in the pathophysiology and treatment of hypertension and related metabolic disorders. Am J Med 93, S11-S20.

169. Liao F, Folsom AR \& Brancati FL (1998) Is low magnesium concentration a risk factor for coronary heart disease? The Atherosclerosis Risk in Communities (ARIC) Study. Am Heart J 136, 480-490.

170. Shechter M, Merz CNB, Paul-Labrador M, et al. (1999) Oral magnesium supplementation inhibits plateletdependent thrombosis in patients with coronary artery disease. Am J Cardiol 84, 152-156.

171. Kawano Y, Matsuoka H, Takishita S, et al. (1998) Effects of magnesium supplementation in hypertensive patients: assessment by office, home, and ambulatory blood pressures. Hypertension 32, 260-265.

172. Al-Mamary M, Al-Habori M, Al-Aghbari A, et al. (2001) In vivo effects of dietary sorghum tannins on rabbit digestive enzymes and mineral absorption. Nutr Res 21, $1393-1401$.

173. Thompson LU (1993) Potential health benefits and problems associated with antinutrients in foods. Food Res Int 26, 131-149.

174. Gillooly M, Bothwell TH, Charlton RW, et al. (1984) Factors affecting the absorption of iron from cereals. $\mathrm{Br} J$ Nutr 51, 37-46.

175. Tatala S, Svanberg U \& Mduma B (1998) Low dietary iron availability is a major cause of anemia: a nutrition survey in the Lindi District of Tanzania. Am J Clin Nutr 68, $171-178$.

176. Lestienne I, Besancon P, Caporiccio B, et al. (2005) Iron and zinc in vitro availability in pearl millet flours (Pennisetum glaucum) with varying phytate, tannin, and fiber contents. J Agric Food Chem 53, 3240-3247.
177. Hassan IAG \& El Tinay AH (1995) Effect of fermentation on tannin content and in-vitro protein and starch digestibilities of two sorghum cultivars. Food Chem 53, 149-151.

178. Matuschek E, Towo E \& Svanberg U (2001) Oxidation of polyphenols in phytate-reduced high-tannin cereals: effect on different phenolic groups and on in vitro accessible iron. J Agric Food Chem 49, 5630-5638.

179. Mbithi-Mwikya S, Van Camp J, Yiru Y, et al. (2000) Nutrient and antinutrient changes in finger millet (Eleusine coracan) during sprouting. Lebensm-Wiss Technol Food Sci Technol 33, 9-14.

180. Towo E, Matuschek E \& Svanberg U (2006) Fermentation and enzyme treatment of tannin sorghum gruels: effects on phenolic compounds, phytate and in vitro accessible iron. Food Chem 94, 369-376.

181. Thompson LU (1988) Antinutrients and blood glucose. Food Technol 42, 123-132.

182. Yoon J, Thompson L \& Jenkins D (1983) The effect of phytic acid on in vitro rate of starch digestibility and blood glucose response. Am J Clin Nutr 38, 835-842.

183. Manach C, Williamson G, Morand C, et al. (2005) Bioavailability and bioefficacy of polyphenols in humans. I. Review of 97 bioavailability studies. Am J Clin Nutr $\mathbf{8 1}$, 230S-242S.

184. Choi J-S, Choi Y-J, Shin S-Y, et al. (2008) Dietary flavonoids differentially reduce oxidized LDL-induced apoptosis in human endothelial cells: role of MAPK- and JAK/STAT-signaling. $J$ Nutr 138, 983-990.

185. Crespo I, García-Mediavilla MV, Gutiérrez B, et al. (2008) A comparison of the effects of kaempferol and quercetin on cytokine-induced pro-inflammatory status of cultured human endothelial cells. Br J Nutr 100, 968-976.

186. Maggi-Capeyron MF, Ceballos P, Cristol JP, et al. (2001) Wine phenolic antioxidants inhibit AP-1 transcriptional activity. J Agric Food Chem 49, 5646-5652.

187. Yun K-J, Koh D-J, Kim S-H, et al. (2008) Antiinflammatory effects of sinapic acid through the suppression of inducible nitric oxide synthase, cyclooxygase-2, and proinflammatory cytokines expressions via nuclear factor- $\kappa \mathrm{B}$ inactivation. $J$ Agric Food Chem 56, $10265-10272$.

188. Moskaug JO, Carlsen H, Myhrstad MC, et al. (2005) Polyphenols and glutathione synthesis regulation. Am J Clin Nutr 81, 277S-283S.

189. Rahman I, Biswas SK \& Kirkham PA (2006) Regulation of inflammation and redox signaling by dietary polyphenols. Biochem Pharmacol 72, 1439-1452.

190. Ramos S (2008) Cancer chemoprevention and chemotherapy: dietary polyphenols and signalling pathways. Mol Nutr Food Res 52, 507-526.

191. Williams RJ, Spencer JPE \& Rice-Evans C (2004) Flavonoids: antioxidants or signalling molecules? Free Radic Biol Med 36, 838-849.

192. McCallum JA \& Walker JRL (1990) Proanthocyanidins in wheat bran. Cereal Chem 67, 282-285.

193. Feng Y \& McDonald CE (1989) Comparison of flavonoids in bran of four classes of wheat. Cereal Chem 66, $516-518$.

194. Gallardo C, Jiménez L \& García-Conesa M-T (2006) Hydroxycinnamic acid composition and in vitro antioxidant activity of selected grain fractions. Food Chem $\mathbf{9 9}$, 455-463.

195. Myhrstad MCW, Carlsen H, Nordstrom O, et al. (2002) Flavonoids increase the intracellular glutathione level by transactivation of the $\gamma$-glutamylcysteine synthetase 
catalytical subunit promoter. Free Radic Biol Med 32, 386-393.

196. Kern SM, Bennett RN, Mellon FA, et al. (2003) Absorption of hydroxycinnamates in humans after high-bran cereal consumption. J Agric Food Chem 51, 6050-6055.

197. Li L, Shewry PR \& Ward JL (2008) Phenolic acids in wheat varieties in the HEALTHGRAIN diversity screen. J Agric Food Chem 56, 9732-9739.

198. Métayer S, Seiliez I, Collin A, et al. (2008) Mechanisms through which sulfur amino acids control protein metabolism and oxidative status. J Nutr Biochem 19 207-215.

199. Tesseraud S, Métayer Coustard S, Collin A, et al. (2009) Role of sulfur amino acids in controlling nutrient metabolism and cell functions: implications for nutrition. Br J Nutr 101, 1132-1139.

200. Morand C, Rios L, Moundras C, et al. (1997) Influence of methionine availability on glutathione synthesis and delivery by the liver. J Nutr Biochem 8, 246-255.

201. Nkabyo YS, Gu LH, Jones DP, et al. (2006) Thiol/disulfide redox status is oxidized in plasma and small intestinal and colonic mucosa of rats with inadequate sulfur amino acid intake. $J$ Nutr 136, 1242-1248.

202. Tateishi N, Hirasawa M, Higashi T, et al. (1982) The L-methionine-sparing effect of dietary glutathione in rats. J Nutr 112, 2217-2226.

203. Flagg EW, Coates RJ, Eley JW, et al. (1994) Dietary glutathione intake in humans and the relationship between intake and plasma total glutathione level. Nutr Cancer 21, 33-46.

204. Martin A (2001) Apports nutritionnels conseillés pour la population française (Recommended Dietary Allowances for the French Population), 3rd ed. Paris: Editions TEC \& DOC.

205. US Department of Agriculture ARS \& Nutrient Data Laboratory (2005) USDA National Nutrient Database for Standard Reference, release 18, Baked products. http://www.nal.usda.gov/fnic/foodcomp/Data/SR18/reports/ sr18page.htm (accessed January 2010).

206. Smith AT, Kuznesof S, Richardson DP, et al. (2003) Behavioural, attitudinal and dietary responses to the consumption of wholegrain foods. Proc Nutr Soc 62, 455-467.

207. Hagen TM, Wierzbicka GT, Bowman BB, et al. (1990) Fate of dietary glutathione: disposition in the gastrointestinal tract. Am J Physiol Gastrointest Liver Physiol 259, G530-G535.

208. Gmünder H, Roth S, Eck H-P, et al. (1990) Interleukin-2 mRNA expression, lymphokine production and DNA synthesis in glutathione-depleted T cells. Cell Immunol 130, 520-528.

209. Witschi A, Reddy S, Stofer B, et al. (1992) The systemic availability of oral glutathione. Eur J Clin Pharmacol 43, 667-669.

210. Sarwin R, Walther C, Laskawy G, et al. (1992) Determination of free reduced and total glutathione in wheat flours by an isotope-dilution assay. Z Lebens Unters Forsch 195, 27-32.

211. Li W, Bollecker SS \& Schofield JD (1995) Glutathione and related thiol compounds. I. Glutathione and related thiol compounds in flour. J Cereal Sci 39, 205-212.

212. Weber F \& Grosch W (1978) Determination of reduced and oxidized glutathione in wheat flours and doughs. Z Lebens Unters Forsch 167, 87-92.

213. Lotito SB \& Frei B (2004) The increase in human plasma antioxidant capacity after apple consumption is due to the metabolic effect of fructose on urate, not apple-derived antioxidant flavonoids. Free Radic Biol Med 37, 251-258.

214. Lotito SB \& Frei B (2006) Consumption of flavonoid-rich foods and increased plasma antioxidant capacity in humans: cause, consequence, or epiphenomenon? Free Radic Biol Med 41, 1727-1746.

215. Souci SW, Fachmann W \& Kraut H (2000) Food Composition and Nutritional Tables. Stuttgart: Medpharm Scientific Publishers.

216. Natella F, Nardini M, Giannetti I, et al. (2002) Coffee drinking influences plasma antioxidant capacity in humans. J Agric Food Chem 50, 6211-6216.

217. Lopez HW, Leenhardt F, Coudray C, et al. (2002) Minerals and phytic acid interactions: is it a real problem for human nutrition? Int J Food Sci Technol 37, 727-739.

218. Graf E \& Eaton GW (1990) Antioxidant functions of phytic acid. Free Radic Biol Med 8, 61-69.

219. Levrat-Verny MA, Coudray C, Bellanger J, et al. (1999) Wholewheat flour ensures higher mineral absorption and bioavailability than white wheat flour in rats. Br J Nutr $\mathbf{8 2}$, 17-21.

220. Leenhardt F, Levrat-Verny MA, Chanliaud E, et al. (2005) Moderate decrease of $\mathrm{pH}$ by sourdough fermentation is sufficient to reduce phytate content of whole wheat flour through endogenous phytase activity. J Agric Food Chem 53, 98-102.

221. Begum AN, Nicolle C, Mila I, et al. (2004) Dietary lignins are precursors of mammalian lignans in rats. J Nutr 134, $120-127$.

222. Kitts DD, Yuan YV, Wijewickreme AN, et al. (1999) Antioxidant activity of the flaxseed lignan secoisolariciresinol diglycoside and its mammalian lignan metabolites enterodiol and enterolactone. Mol Cell Biochem 202, 91-100.

223. Bach Knudsen KE, Serena A, Kjaer AKB, et al. (2003) Rye bread in the diet of pigs enhances the formation of enterolactone and increases its levels in plasma, urine and feces. J Nutr 133, 1368-1375.

224. Labaj J, Wsolova L, Lazarova M, et al. (2004) Repair of oxidative DNA lesions in blood lymphocytes isolated from Sprague-Dawley rats; the influence of dietary intake of lignin. Neoplasma 51, 450-455.

225. Craig SAS (2004) Betaine in human nutrition. Am J Clin Nutr 80, 539-549.

226. Zeisel SH \& Blusztajn JK (1994) Choline and human nutrition. Annu Rev Nutr 14, 269-296.

227. Likes R, Madl RL, Zeisel SH, et al. (2007) The betaine and choline content of a whole wheat flour compared to other mill streams. J Cereal Sci 46, 93-95.

228. Cho S, Johnson G \& Song WO (2002) Folate content of foods: comparison between databases compiled before and after new FDA fortification requirements. J Food Comp Anal 15, 293-307.

229. Bertram HC, Bach Knudsen KE, Serena A, et al. (2006) NMR-based metabonomic studies reveal changes in the biochemical profile of plasma and urine from pigs fed high-fibre rye bread. Br J Nutr 95, 955-962.

230. Fardet A, Canlet C, Gottardi G, et al. (2007) Whole grain and refined wheat flours show distinct metabolic profiles in rats as assessed by a ${ }^{1} \mathrm{H}$ NMR-based metabonomic approach. J Nutr 4, 923-929.

231. Borgschulte G, Kathirvel E, Herrera M, et al. (2008) Betaine treatment reverses insulin resistance and fatty liver disease without reducing oxidative stress or endoplasmic reticulum stress in an animal model of NAFLD. Gastroenterology 134, A414-A415. 
232. Brouwer IA, van Dusseldorp M, Thomas CM, et al. (1999) Low-dose folic acid supplementation decreases plasma homocysteine concentrations: a randomized trial. Am J Clin Nutr 69, 99-104.

233. Graham IM, Daly LE, Refsum HM, et al. (1997) Plasma homocysteine as a risk factor for vascular disease. The European Concerted Action Project. JAMA 277, $1775-1781$.

234. Mills JL, McPartlin JM, Kirke PN, et al. (1995) Homocysteine metabolism in pregnancies complicated by neural-tube defects. Lancet 345, 149-151.

235. Wu LL \& Wu JT (2002) Hyperhomocysteinemia is a risk factor for cancer and a new potential tumor marker. Clin Chim Acta 322, 21-28.

236. Loscalzo J (1996) The oxidant stress of hyperhomocys$\mathrm{t}(\mathrm{e})$ inemia. $J$ Clin Invest 98, 5-7.

237. Tyagi N, Sedoris KC, Steed M, et al. (2005) Mechanisms of homocysteine-induced oxidative stress. Am J Physiol Heart Circ Physiol 289, H2649-H2656.

238. Christman JK, Chen M-L, Sheikhnejad G, et al. (1993) Methyl deficiency, DNA methylation, and cancer: studies on the reversibility of the effects of a lipotrope-deficient diet. J Nutr Biochem 4, 672-680.

239. Newberne PM \& Rogers AE (1986) Labile methyl groups and the promotion of cancer. Annu Rev Nutr 6, 407-432.

240. Zeisel S, Da Costa K, Franklin P, et al. (1991) Choline, an essential nutrient for humans. FASEB J 5, 2093-2098.

241. Iqbal TH, Lewis KO \& Cooper BT (1994) Phytase activity in the human and rat small intestine. Gut 35, 1233-1236.

242. Okazaki Y, Setoguchi T \& Katayama T (2006) Effects of dietary myo-inositol, D-chiro-inositol and L-chiro-inositol on hepatic lipids with reference to the hepatic myo-inositol status in rats fed on 1,1,1-trichloro-2,2-bis (p-chlorophenyl) ethane. Biosci Biotechnol Biochem 70, 2766-2770.

243. Horbowicz M \& Obendorf RL (2005) Fagopyritol accumulation and germination of buckwheat seeds matured at 15,22 , and $30^{\circ}$ C. Crop Sci 45, 1264-1270.

244. Steadman KJ, Burgoon MS, Schuster RL, et al. (2000) Fagopyritols, D-chiro-inositol, and other soluble carbohydrates in buckwheat seed milling fractions. J Agric Food Chem 48, 2843-2847.

245. Kim JI, Kim JC, Joo HJ, et al. (2005) Determination of total chiro-inositol content in selected natural materials and evaluation of the antihyperglycemic effect of pinitol isolated from soybean and carob. Food Sci Biotechnol 14, 441-445.

246. Becker R, Wheeler EL, Lorenz K, et al. (1981) A compositional study of amaranth grain. J Food Sci 46, $1175-1180$

247. Darbre A \& Norris FW (1956) Vitamins in germination determination of free and combined inositol in germinating oats. Biochem J 64, 441-446.

248. Koziol MJ (1992) Chemical composition and nutritional evaluation of quinoa (Chenopodium quinoa Willd.). J Food Comp Anal 5, 35-68.

249. Horbowicz M \& Obendorf RL (1994) Seed desiccation tolerance and storability: dependence on flatulenceproducing oligosaccharides and cyclitols? - review and survey. Seed Sci Res 4, 385-405.

250. Matheson NK \& Strother S (1969) The utilization of phytate by germinating wheat. Phytochemistry 8 , $1349-1356$

251. Clements R Jr \& Darnell B (1980) Myo-inositol content of common foods: development of a high-myo-inositol diet. Am J Clin Nutr 33, 1954-1967.

252. Reddy NR, Sathe SK \& Salunkhe DK (1982) Phytates in legumes and cereals. Adv Food Res 28, 1-92.
253. Ferrel RE (1978) Distribution of bean and wheat inositol phosphate esters during autolysis and germination. J Food Sci 43, 563-565.

254. Nakano T, Joh T, Narita K, et al. (2000) The pathway of dephosphorylation of myo-inositol hexakisphosphate by phytases from wheat bran of Triticum aestivum L. cv. Nourin \#61. Biosci Biotechnol Biochem 64, 995-1003.

255. Bergman E-L, Fredlund K, Reinikainen P, et al. (1999) Hydrothermal processing of barley (cv. Blenheim): optimisation of phytate degradation and increase of free myo-inositol. J Cereal Sci 29, 261-272.

256. Pak Y, Huang L, Lilley K, et al. (1992) In vivo conversion of $\left[{ }^{3} \mathrm{H}\right]$ myoinositol to $\left[{ }^{3} \mathrm{H}\right]$ chiroinositol in rat tissues. J Biol Chem 267, 16904-16910.

257. Reeves PG, Nielsen FH \& Fahey GC Jr (1993) AIN-93 purified diets for laboratory rodents: final report of the American Institute of Nutrition ad hoc writing committee on the reformulation of the AIN-76A rodent diet. $J$ Nutr 123, 1939-1951.

258. Locker J, Reddy TV \& Lombardi B (1986) DNA methylation and hepatocarcinogenesis in rats fed a choline-devoid diet. Carcinogenesis 7, 1309-1312.

259. Gama-Sosa MA, Slagel VA, Trewyn RW, et al. (1983) The 5-methylcytosine content of DNA from human tumors. Nucleic Acids Res 11, 6883-6894.

260. Goelz S, Vogelstein B, Hamilton SR, et al. (1985) Hypomethylation of DNA from benign and malignant human colon neoplasms. Science 228, 187-190.

261. Ou SY \& Kwok KC (2004) Ferulic acid: pharmaceutical functions, preparation and applications in foods. J Sci Food Agric 84, 1261-1269.

262. Srinivasan M, Sudheer AR \& Menon VP (2007) Ferulic acid: therapeutic potential through its antioxidant property. $J$ Clin Biochem Nutr 40, 92-100.

263. Rybka K, Sitarski J \& Raczynskabojanowska K (1993) Ferulic acid in rye and wheat-grain and grain dietary fiber. Cereal Chem 70, 55-59.

264. Kroon PA, Faulds CB, Ryden P, et al. (1997) Release of covalently bound ferulic acid from fiber in the human colon. J Agric Food Chem 45, 661-667.

265. Akao Y, Seki N, Nakagawa Y, et al. (2004) A highly bioactive lignophenol derivative from bamboo lignin exhibits a potent activity to suppress apoptosis induced by oxidative stress in human neuroblastoma SH-SY5Y cells. Bioorg Med Chem 12, 4791-4801.

266. Ferguson LR \& Harris PJ (1996) Studies on the role of specific dietary fibres in protection against colorectal cancer. Mutat Res 350, 173-184.

267. Calvert GD \& Yeates RA (1982) Adsorption of bile salts by soya-bean flour, wheat bran, lucerne (Medicago sativa), sawdust and lignin: the effect of saponins and other plant constituents. Br J Nutr 47, 45-52.

268. Chang MLW \& Johnson MA (1980) Effect of lignin versus cellulose on the absorption of taurocholate and lipid metabolism in rats fed cholesterol diet. Nutr Rep Int $\mathbf{2 1}$ $513-518$

269. Drasar B \& Jenkins D (1976) Bacteria, diet, and large bowel cancer. Am J Clin Nutr 29, 1410-1416.

270. Anjaneyulu M \& Chopra K (2004) Nordihydroguairetic acid, a lignin, prevents oxidative stress and the development of diabetic nephropathy in rats. Pharmacology 72, 42-50.

271. Fardet A, Llorach R, Orsoni A, et al. (2008) Metabolomics provide new insight on the metabolism of dietary phytochemicals in rats. $J$ Nutr 138, 1282-1287. 
272. Hindmarch I (2002) Beyond the monoamine hypothesis: mechanisms, molecules and methods. Eur Psychiatry 17, 294-299.

273. Berry RJ, Li Z, Erickson JD, et al. (1999) Prevention of neural-tube defects with folic acid in China. $N$ Engl J Med 341, 1485-1490.

274. Coppen A \& Bolander-Gouaille C (2005) Treatment of depression: time to consider folic acid and vitamin $\mathrm{B}_{12}$. J Psychopharmacol 19, 59-65.

275. Miller AL (2008) The methylation, neurotransmitter, and antioxidant connections between folate and depression. Altern Med Rev 13, 216-226.

276. Gilbody S, Lightfoot T \& Sheldon T (2007) Is low folate a risk factor for depression? A meta-analysis and exploration of heterogeneity. J Epidemiol Community Health 61, 631-637.

277. Lioger D, Leenhardt F, Demigne C, et al. (2007) Sourdough fermentation of wheat fractions rich in fibres before their use in processed food. J Sci Food Agric 87, $1368-1373$.

278. Ohta A, Ohtsuki M, Hosono A, et al. (1998) Dietary fructooligosaccharides prevent osteopenia after gastrectomy in rats. $J$ Nutr 128, 106-110.

279. Scholz-Ahrens KE, Ade P, Marten B, et al. (2007) Prebiotics, probiotics, and synbiotics affect mineral absorption, bone mineral content, and bone structure. J Nutr 137, 838S-846S.

280. Scholz-Ahrens KE \& Schrezenmeir J (2007) Inulin and oligofructose and mineral metabolism: the evidence from animal trials. J Nutr 137, 2513S-2523S

281. Nordbö H \& Rolla G (1972) Desorption of salivary proteins from hydroxyapatite by phytic acid and glycerophosphate and the plaque-inhibiting effect of the two compounds in vivo. J Dent Res 51, 800-802.

282. McClure FJ (1964) Cariostatic effect of phosphates. Science 144, 1337-1338.

283. Osborn TWB \& Noriskin JN (1937) The relation between diet and caries in the South African Bantu. J Dent Res 16, $431-441$.

284. Osborn TWB, Noriskin JN \& Staz J (1937) A comparison of crude and refined sugar and cereals in their ability to produce in vitro decalcification of teeth. J Dent Res 16, $165-171$.

285. Osborn TWB (1941) Further studies on the in vitro decalcification of teeth. J Dent Res 20, 59-69.

286. McClure FJ (1960) The cariostatic effect in white rats of phosphorus and calcium supplements added to the flour of bread formulas and to bread diets. J Nutr 72, 131-136.

287. McClure FJ (1963) Further studies on the cariostatic effect of organic and inorganic phosphates. J Dent Res 42, 693-699.

288. McClure FJ \& Muller A Jr (1959) Further observations on the cariostatic effect of phosphates. J Dent Res 38, 776-781.

289. Wynn W, Haldi J, Bentley KD, et al. (1956) Dental caries in the albino rat in relation to the chemical composition of the teeth and of the diet: II. Variations in the $\mathrm{Ca} / \mathrm{P}$ ratio of the diet induced by changing the phosphorus content J Nutr 58, 325-333.

290. Magrill DS (1973) The reduction of the solubility of hydroxyapatite in acid by adsorption of phytate from solution. Arch Oral Biol 18, 591-600.

291. Pruitt KM, Jamieson AD \& Caldwell RC (1970) Possible basis for the cariostatic effect of inorganic phosphates. Nature 225, 1249.

292. Cole MF \& Bowen WH (1975) Effect of sodium phytate on the chemical and microbial composition of dental plaque in the monkey (Macaca fascicularis). J Dent Res 54, 449-457.

293. Adlercreutz H \& Mazur W (1997) Phyto-oestrogens and Western diseases. Ann Med 29, 95-120.

294. Chanvrier H, Appelqvist IA, Bird AR, et al. (2007) Processing of novel elevated amylose wheats: functional properties and starch digestibility of extruded products. J Agric Food Chem 55, 10248-10257.

295. Swennen K, Courtin CM \& Delcour JA (2006) Nondigestible oligosaccharides with prebiotic properties. Crit Rev Food Sci Nutr 46, 459-471.

296. Krause DO, Easter RA \& Mackie RI (1994) Fermentation of stachyose and raffinose by hind-gut bacteria of the weanling pig. Lett Appl Microbiol 18, 349-352.

297. Tortuero F, Fernández E, Rupérez P, et al. (1997) Raffinose and lactic bacteria influence caecal fermentation and serum cholesterol in rats. Nutr Res 17, 41-49.

298. Alessandri C, Pignatelli P, Loffredo L, et al. (2006) $\alpha$-Linolenic acid-rich wheat germ oil decreases oxidative stress and CD40 ligand in patients with mild hypercholesterolemia. Arterioscler Thromb Vasc Biol 26, 2577-2578.

299. Farquhar JW, Smith RE \& Dempsey ME (1956) The effect of beta sitosterol on the serum lipids of young men with arteriosclerotic heart disease. Circulation 14, 77-82.

300. Jones PJ, Ntanios FY, Raeini-Sarjaz M, et al. (1999) Cholesterol-lowering efficacy of a sitostanol-containing phytosterol mixture with a prudent diet in hyperlipidemic men. Am J Clin Nutr 69, 1144-1150.

301. Kato S, Karino K-I, Hasegawa S, et al. (1995) Octacosanol affects lipid metabolism in rats fed on a high-fat diet. $\mathrm{Br} J$ Nutr 73, 433-441.

302. Taylor JC, Rapport L \& Lockwood GB (2003) Octacosanol in human health. Nutrition 19, 192-195.

303. Gouni-Berthold I \& Berthold HK (2002) Policosanol: clinical pharmacology and therapeutic significance of a new lipid-lowering agent. Am Heart J 143, 356-365.

304. Varady KA, Wang Y \& Jones PJH (2003) Role of policosanols in the prevention and treatment of cardiovascular disease. Nutr Rev 61, 376-383.

305. Lin YG, Rudrum M, van der Wielen RPJ, et al. (2004) Wheat germ policosanol failed to lower plasma cholesterol in subjects with normal to mildly elevated cholesterol concentrations. Metabolism 53, 1309-1314.

306. Menendez R, Arruzazabala L, Mas R, et al. (1997) Cholesterol-lowering effect of policosanol on rabbits with hypercholesterolaemia induced by a wheat starch-casein diet. Br J Nutr 77, 923-932.

307. Menendez R, Fraga V, Amor AM, et al. (1999) Oral administration of policosanol inhibits in vitro copper ioninduced rat lipoprotein peroxidation. Physiol Behav 67, $1-7$.

308. Hosseinian FS, Li W \& Beta T (2008) Measurement of anthocyanins and other phytochemicals in purple wheat. Food Chem 109, 916-924.

309. Asayama K, Yamadera H, Ito T, et al. (2003) Double blind study of melatonin effects on the sleep-wake rhythm, cognitive and non-cognitive functions in Alzheimer type dementia. J Nippon Med Sch 70, 334-341.

310. Maurizi CP (2001) Alzheimer's disease: roles for mitochondrial damage, the hydroxyl radical, and cerebrospinal fluid deficiency of melatonin. Med Hypotheses 57, 156-160.

311. Garcia-Navarro A, Gonzalez-Puga C, Escames G, et al. (2007) Cellular mechanisms involved in the melatonin inhibition of HT-29 human colon cancer cell proliferation in culture. J Pineal Res 43, 195-205. 
312. Shiu SYW (2007) Towards rational and evidence-based use of melatonin in prostate cancer prevention and treatment. J Pineal Res 43, 1-9.

313. Calhoun WK, Bechtel WG \& Bradley WB (1958) The vitamin content of wheat, flour, and bread. Cereal Chem 35, 350-359.

314. Calhoun WK, Hepburn FN \& Bradley WB (1960) The distribution of the vitamins of wheat in commercial mill products. Cereal Chem 37, 755-761.

315. Wang LH, Huang WS \& Tai HM (2007) Simultaneous determination of $p$-aminobenzoic acid and its metabolites in the urine of volunteers, treated with $p$-aminobenzoic acid sunscreen formulation. J Pharm Biomed Anal 43, 1430-1436.

316. Barbieri B, Papadogiannakis N, Eneroth P, et al. (1995) Arachidonic acid is a preferred acetyl donor among fatty acids in the acetylation of $p$-aminobenzoic acid by human lymphoid cells. Biochim Biophys Acta 1257, 157-166.

317. Failey RB \& Childress RH (1962) The effect of paraaminobenzoic acid on the serum cholesterol level in man. Am J Clin Nutr 10, 158-162.

318. Butcher NJ, Ilett KF \& Minchin RF (2000) Inactivation of human arylamine $N$-acetyltransferase 1 by the hydroxylamine of $p$-aminobenzoic acid. Biochem Pharmacol 60, 1829-1836.

319. Hein DW, Doll MA, Gray K, et al. (1993) Metabolic activation of $N$-hydroxy-2-aminofluorene and $N$-hydroxy2-acetylaminofluorene by monomorphic $N$-acetyltransferase (NAT1) and polymorphic $N$-acetyltransferase (NAT2) in colon cytosols of syrian hamsters congenic at the NAT2 locus. Cancer Res 53, 509-514.

320. Minchin RF, Reeves PT, Teitel CH, et al. (1992) $N$ - and $O$-acetylation of aromatic and heterocyclic amine carcinogens by human monomorphic and polymorphic acetyltransferases expressed in COS-1 cells. Biochem Biophys Res Commun 185, 839-844.

321. Barbieri B, Papadogiannakis N, Eneroth P, et al. (1997) $p$-Aminobenzoic acid, but not its metabolite $p$-acetamidobenzoic acid, inhibits thrombin induced thromboxane formation in human platelets in a non NSAID like manner. Thromb Res 86, 127-140.

322. Elliott R, Pico C, Dommels Y, et al. (2007) Nutrigenomic approaches for benefit-risk analysis of foods and food components: defining markers of health. Br J Nutr 98, 1095-1100.

323. Steiner C, Arnould S, Scalbert A, et al. (2008) Isoflavones and the prevention of breast and prostate cancer: new perspectives opened by nutrigenomics. Br J Nutr 99, E Suppl. 1, ES78-ES108.

324. Trujillo E, Davis C \& Milner J (2006) Nutrigenomics, proteomics, metabolomics, and the practice of dietetics. J Am Diet Assoc 106, 403-413.

325. van Ommen B (2004) Nutrigenomics: exploiting systems biology in the nutrition and health arenas. Nutrition 20, 4-8.

326. Zeisel SH (2007) Nutrigenomics and metabolomics will change clinical nutrition and public health practice: insights from studies on dietary requirements for choline. Am J Clin Nutr 86, 542-548.

327. Keun HC (2006) Metabonomic modeling of drug toxicity. Pharmacol Ther 109, 92-106.

328. Rezzi S, Ramadan Z, Fay LB, et al. (2007) Nutritional metabonomics: applications and perspectives. J Proteome Res 6, 513-525.

329. Selman C, Kerrison ND, Cooray A, et al. (2006) Coordinated multitissue transcriptional and plasma metabonomic profiles following acute caloric restriction in mice. Physiol Genomics 27, 187-200.

330. Griffin JL, Muller D, Woograsingh R, et al. (2002) Vitamin E deficiency and metabolic deficits in neuronal ceroid lipofuscinosis described by bioinformatics. Physiol Genomics 11, 195-203.

331. Mutch DM, Grigorov M, Berger A, et al. (2005) An integrative metabolism approach identifies stearoyl-CoA desaturase as a target for an arachidonate-enriched diet. FASEB J 19, 599-619.

332. Solanky KS, Bailey NJC, Beckwith-Hall BM, et al. (2003) Application of biofluid H-1 nuclear magnetic resonancebased metabonomic techniques for the analysis of the biochemical effects of dietary isoflavones on human plasma profile. Anal Biochem 323, 197-204.

333. Wang Y, Tang H, Nicholson JK, et al. (2005) A metabonomic strategy for the detection of the metabolic effects of chamomile (Matricaria recutita L.) ingestion. J Agric Food Chem 53, 191-196.

334. Van Dorsten FA, Daykin CA, Mulder TPJ, et al. (2006) Metabonomics approach to determine metabolic differences between green tea and black tea consumption. J Agric Food Chem 54, 6929-6938.

335. Solanky KS, Bailey NJC, Holmes E, et al. (2003) NMRbased metabonomic studies on the biochemical effects of epicatechin in the rat. J Agric Food Chem 51, 4139-4145.

336. Fardet A, Llorach R, Martin JF, et al. (2008) A liquid chromatography-quadrupole time-of-flight (LC-QTOF)based metabolomic approach reveals new metabolic effects of catechin in rats fed high-fat diets. $J$ Proteome Res 7, 2388-2398.

337. Dumas ME, Barton RH, Toye A, et al. (2006) Metabolic profiling reveals a contribution of gut microbiota to fatty liver phenotype in insulin-resistant mice. Proc Natl Acad Sci U S A 103, 12511-12516.

338. Zhang S, Nagana Gowda GA, Asiago V, et al. (2008) Correlative and quantitative ${ }^{1} \mathrm{H}$ NMR-based metabolomics reveals specific metabolic pathway disturbances in diabetic rats. Anal Biochem 383, 76-84.

339. Britz SJ, Prasad PVV, Moreau RA, et al. (2007) Influence of growth temperature on the amounts of tocopherols, tocotrienols, and $\gamma$-oryzanol in brown rice. J Agric Food Chem 55, 7559-7565.

340. Packer L, Witt EH \& Tritschler HJ (1995) $\alpha$-Lipoic acid as a biological antioxidant. Free Radic Biol Med 19, 227-250.

341. Roy S, Sen CK, Tritschler HJ, et al. (1997) Modulation of cellular reducing equivalent homeostasis by $\alpha$-lipoic acid: mechanisms and implications for diabetes and ischemic injury. Biochem Pharmacol 53, 393-399.

342. Maczurek A, Hager K, Kenklies M, et al. (2008) Lipoic acid as an anti-inflammatory and neuroprotective treatment for Alzheimer's disease. Adv Drug Deliv Rev 60, $1463-1470$.

343. Wollin SD \& Jones PJH (2003) $\alpha$-Lipoic acid and cardiovascular disease. J Nutr 133, 3327-3330.

344. Yu SG, Nehus ZT, Badger TM, et al. (2007) Quantification of vitamin $\mathrm{E}$ and $\gamma$-oryzanol components in rice germ and bran. J Agric Food Chem 55, 7308-7313.

345. Emmons CL, Peterson DM \& Paul GL (1999) Antioxidant capacity of oat (Avena sativa L.) extracts. 2. In vitro antioxidant activity and contents of phenolic and tocol antioxidants. J Agric Food Chem 47, 4894-4898.

346. Heinemann T, Kullak-Ublick G-A, Pietruck B, et al (1991) Mechanisms of action of plant sterols on inhibition of cholesterol absorption. Eur J Clin Pharmacol 40, S59-S63. 
347. Sen CK, Khanna S \& Roy S (2006) Tocotrienols: vitamin E beyond tocopherols. Life Sci 78, 2088-2098.

348. Wilson TA, Nicolosi RJ, Woolfrey B, et al. (2007) Rice bran oil and oryzanol reduce plasma lipid and lipoprotein cholesterol concentrations and aortic cholesterol ester accumulation to a greater extent than ferulic acid in hypercholesterolemic hamsters. J Nutr Biochem 18, $105-112$.

349. Kahlon TS \& Chow FI (2000) In vitro binding of bile acids by rice bran, oat bran, wheat bran, and corn bran. Cereal Chem 77, 518-521.

350. Saulnier L, Vigouroux J \& Thibault J-F (1995) Isolation and partial characterization of feruloylated oligosaccharides from maize bran. Carbohydr Res 272, 241-253.

351. Saulnier L, Marot C, Elgorriaga M, et al. (2001) Thermal and enzymatic treatments for the release of free ferulic acid from maize bran. Carbohydr Polym 45, 269-275.

352. O'Dell BL, De Boland AR \& Koityonann SR (1972) Distribution of phytate and nutritionally important elements among the morphological components of cereals grains. J Agric Food Chem 20, 718-721.

353. Miller A \& Engel KH (2006) Content of $\gamma$-oryzanol and composition of steryl ferulates in brown rice (Oryza sativa L.) of European origin. J Agric Food Chem 54, 8127-8133.

354. Chen MH \& Bergman CJ (2005) A rapid procedure for analysing rice bran tocopherol, tocotrienol and $\gamma$-oryzanol contents. J Food Comp Anal 18, 139-151.

355. Schramm R, Abadie A, Hua N, et al. (2007) Fractionation of the rice bran layer and quantification of vitamin $\mathrm{E}$, oryzanol, protein, and rice bran saccharide. J Biol Eng 1, 9.

356. Shin T-S, Godber JS, Martin DE, et al. (1997) Hydrolytic stability and changes in E vitamers and oryzanol of extruded rice bran during storage. J Food Sci 62, 704-728.

357. Juliano C, Cossu M, Alamanni MC, et al. (2005) Antioxidant activity of $\gamma$-oryzanol: mechanism of action and its effect on oxidative stability of pharmaceutical oils. Int J Pharm 299, 146-154.

358. Rong N, Ausman L \& Nicolosi R (1997) Oryzanol decreases cholesterol absorption and aortic fatty streaks in hamsters. Lipids 32, 303-309.

359. Seetharamaiah GS \& Chandrasekhara N (1993) Comparative hypocholesterolemic activities of oryzanol, curcumin and ferulic acid in rats. J Food Sci Technol Mysore 30, $249-252$.

360. Suh MH, Yoo SH, Chang PS, et al. (2005) Antioxidative activity of microencapsulated $\gamma$-oryzanol on high cholesterol-fed rats. J Agric Food Chem 53, 9747-9750.

361. Cicero AFG \& Gaddi A (2001) Rice bran oil and $\gamma$-oryzanol in the treatment of hyperlipoproteinaemias and other conditions. Phytother Res 15, 277-289.

362. Collins FW (1989) Oat phenolics: avenanthramides, novel substituted $\mathrm{N}$-cinnamoylanthranilate alkaloids from oat groats and hulls. J Agric Food Chem 37, 60-66.

363. Shewry PR, Piironen V, Lampi A-M, et al. (2008) Phytochemical and fiber components in oat varieties in the HEALTHGRAIN diversity screen. J Agric Food Chem 56, 9777-9784.

364. Dimberg LH, Theander O \& Lingnert H (1993) Avenanthramides - a group of phenolic antioxidants in oats. Cereal Chem 70, 637-641.

365. Mattila P, Pihlava J-M \& Hellstrom J (2005) Contents of phenolic acids, alkyl- and alkenylresorcinols, and avenanthramides in commercial grain products. J Agric Food Chem 53, 8290-8295.
366. Fagerlund A, Sunnerheim K \& Dimberg LH (2009) Radical-scavenging and antioxidant activity of avenanthramides. Food Chem 113, 550-556.

367. Peterson DM, Hahn MJ \& Emmons CL (2002) Oat avenanthramides exhibit antioxidant activities in vitro. Food Chem 79, 473-478.

368. Liu L, Zubik L, Collins FW, et al. (2004) The antiatherogenic potential of oat phenolic compounds. Atherosclerosis 175, 39-49.

369. Chen CY, Milbury PE, Kwak HK, et al. (2004) Avenanthramides and phenolic acids from oats are bioavailable and act synergistically with vitamin $\mathrm{C}$ to enhance hamster and human LDL resistance to oxidation. J Nutr 134, 1459-1466.

370. Güçlü-Üstündag Ö \& Mazza G (2007) Saponins: properties, applications and processing. Crit Rev Food Sci Nutr 47, 231-258.

371. Osbourn AE (2003) Saponins in cereals. Phytochemistry 62, 1-4.

372. Önning G, Asp N-G \& Sivik B (1993) Saponin content in different oat varieties and in different fractions of oat grain. Food Chem 48, 251-254.

373. Price KR, Johnson IT \& Fenwick GR (1987) The chemistry and biological significance of saponins in foods and feedingstuffs. Crit Rev Food Sci Nutr 26, $27-135$.

374. Matsuura H (2001) Saponins in garlic as modifiers of the risk of cardiovascular disease. J Nutr 131, 1000S-1005S.

375. Barr IG, Sjölander A \& Cox JC (1998) ISCOMs and other saponin based adjuvants. Adv Drug Deliv Rev 32, 247-271.

376. Sjölander A, Cox J \& Barr I (1998) ISCOMs: an adjuvant with multiple functions. J Leukoc Biol 64, 713-723.

377. Oakenfull DG, Fenwick DE, Hood RL, et al. (1979) Effects of saponins on bile acids and plasma lipids in the rat. Br J Nutr 42, 209-216.

378. Baik B-K \& Ullrich SE (2008) Barley for food: characteristics, improvement, and renewed interest. J Cereal Sci 48, 233-242.

379. Åman P \& Graham H (1987) Analysis of total and insoluble mixed-linked $(1 \rightarrow 3),(1 \rightarrow 4)-\beta$-D-glucans in barley and oats. J Agric Food Chem 35, 704-709.

380. Anker-Nilssen K, Sahlstrøm S, Knutsen SH, et al. (2008) Influence of growth temperature on content, viscosity and relative molecular weight of water-soluble $\beta$-glucans in barley (Hordeum vulgare L.). J Cereal Sci 48, 670-677.

381. Gajdosová A, Petruláková Z, Havrlentová M, et al. (2007) The content of water-soluble and water-insoluble $\beta$-D-glucans in selected oats and barley varieties. Carbohydr Polym 70, 46-52.

382. Holtekjølen AK, Uhlen AK, Bråthen E, et al. (2006) Contents of starch and non-starch polysaccharides in barley varieties of different origin. Food Chem 94, 348-358.

383. Izydorczyk MS \& Dexter JE (2008) Barley $\beta$-glucans and arabinoxylans: molecular structure, physicochemical properties, and uses in food products - a review. Food Res Int 41, 850-868.

384. Izydorczyk MS, Macri LJ \& MacGregor AW (1998) Structure and physicochemical properties of barley nonstarch polysaccharides - II. Alkaliextractable $\beta$-glucans and arabinoxylans. Carbohydr Polym 35, 259-269.

385. Izydorczyk MS, Storsley J, Labossiere D, et al. (2000) Variation in total and soluble $\beta$-glucan content in hulless barley: effects of thermal, physical, and enzymic treatments. J Agric Food Chem 48, 982-989. 
386. Prentice N, Babler S \& Faber S (1980) Enzymic analysis of $\beta$-D-glucans in cereal grains. Cereal Chem 57, 198-202.

387. Kim H, Stote KS, Behall KM, et al. (2009) Glucose and insulin responses to whole grain breakfasts varying in soluble fiber, $\beta$-glucan: a doase response study in obese women with increased risk for insulin resistance. Eur $J$ Nutr 48, 170-175.

388. Butt MS, Tahir-Nadeem M, Khan MKI, et al. (2008) Oat: unique among the cereals. Eur J Nutr 47, 68-79.

389. Kalra S \& Joad S (2000) Effect of dietary barley $\beta$-glucan on cholesterol and lipoprotein fractions in rats. $J$ Cereal Sci 31, 141-145.

390. Hlebowicz J, Darwiche G, Bjorgell O, et al. (2008) Effect of muesli with $4 \mathrm{~g}$ oat $\beta$-glucan on postprandial blood glucose, gastric emptying and satiety in healthy subjects: a randomized crossover trial. J Am Coll Nutr 27, 470-475.

391. Mantovani MS, Bellini MF, Angeli JPF, et al. (2008) $\beta$-Glucans in promoting health: prevention against mutation and cancer. Mutat Res 658, 154-161.

392. Ross AB, Shepherd MJ, Schupphaus M, et al. (2003) Alkylresorcinols in cereals and cereal products. J Agric Food Chem 51, 4111-4118.

393. Ross AB, Kamal-Eldin A, Lundin EA, et al. (2003) Cereal alkylresorcinols are absorbed by humans. J Nutr 133, 2222-2224.

394. Landberg R, Aman P, Friberg LE, et al. (2009) Dose response of whole-grain biomarkers: alkylresorcinols in human plasma and their metabolites in urine in relation to intake. Am J Clin Nutr 89, 290-296.

395. Linko-Parvinen A-M, Landberg R, Tikkanen MJ, et al. (2007) Alkylresorcinols from whole-grain wheat and rye are transported in human plasma lipoproteins. J Nutr 137, $1137-1142$

396. Ross AB, Kamal-Eldin A \& Aman P (2004) Dietary alkylresorcinols: absorption, bioactivities, and possible use as biomarkers of whole-grain wheat- and rye-rich foods. Nutr Rev 62, 81-95.

397. Guyman LA, Adlercreutz H, Koskela A, et al. (2008) Urinary 3-(3,5-dihydroxyphenyl)-1-propanoic acid, an alkylresorcinol metabolite, is a potential biomarker of whole-grain intake in a U.S. population. J Nutr 138, 1957-1962.

398. Tsuge N, Mizokami M, Imai S, et al. (1992) Adipostatin-A and adipostatin-B, new inhibitors of glycerol-3-phosphate dehydrogenase. J Antibiot 45, 886-891.

399. Ross AB, Chen Y, Frank J, et al. (2004) Cereal alkylresorcinols elevate $\gamma$-tocopherol levels in rats and inhibit $\gamma$-tocopherol metabolism in vitro. J Nutr 134, 506-510.

400. Kozubek A \& Tyman JHP (1999) Resorcinolic lipids, the natural non-isoprenoid phenolic amphiphiles and their biological activity. Chem Rev 99, 1-25.

401. Ross JA \& Kasum CM (2002) Dietary flavonoids: bioavailability, metabolic effects, and safety. Annu Rev Nutr 22, 19-34.

402. Norton I, Moore S \& Fryer P (2007) Understanding food structuring and breakdown: engineering approaches to obesity. Obes Rev 8, Suppl. 1, 83-88.

403. Parada J \& Aguilera JM (2007) Food microstructure affects the bioavailability of several nutrients. J Food Sci 72, R21-R32.

404. Tedeschi C, Clement V, Rouvet M, et al. (2009) Dissolution tests as a tool for predicting bioaccessibility of nutrients during digestion. Food Hydrocolloids 23, $1228-1235$
405. Englyst H, Kingman S \& Cummings J (1992) Classification and measurement of nutritionally important starch fractions. Eur J Clin Nutr 46, S33-S50.

406. Lehmann U \& Robin F (2007) Slowly digestible starch its structure and health implications: a review. Trends Food Sci Technol 18, 346-355.

407. Marangoni A, Idziak S \& Rush J (2008) Controlled release of food lipids using monoglyceride gel phases regulates lipid and insulin metabolism in humans. Food Biophys $\mathbf{3}$, $241-245$.

408. Remond D, Machebeuf M, Yven C, et al. (2007) Postprandial whole-body protein metabolism after a meat meal is influenced by chewing efficiency in elderly subjects. Am J Clin Nutr 85, 1286-1292.

409. Armand M, Pasquier B, Andre M, et al. (1999) Digestion and absorption of 2 fat emulsions with different droplet sizes in the human digestive tract. Am J Clin Nutr 70, 1096-1106.

410. Giovannucci E, Rimm EB, Ascherio A, et al. (1995) Alcohol, low-methionine-low-folate diets, and risk of colon cancer in men. $J$ Natl Cancer Inst 87, 265-273.

411. Lu ZX, Walker KZ, Muir JG, et al. (2000) Arabinoxylan fiber, a byproduct of wheat flour processing, reduces the postprandial glucose response in normoglycemic subjects. Am J Clin Nutr 71, 1123-1128.

412. Moore MA, Park CB \& Tsuda H (1998) Soluble and insoluble fiber influences on cancer development. Crit Rev Oncol Hematol 27, 229-242.

413. Björck I \& Asp N-G (1994) Controlling the nutritional properties of starch in foods - a challenge to the food industry. Trends Food Sci Technol 5, 213-218.

414. Dongowski G, Jacobasch G \& Schmiedl D (2005) Structural stability and prebiotic properties of resistant starch type 3 increase bile acid turnover and lower secondary bile acid formation. J Agric Food Chem $\mathbf{5 3}$, 9257-9267.

415. Topping DL, Fukushima M \& Bird AR (2003) Resistant starch as a prebiotic and synbiotic: state of the art. Proc Nutr Soc 62, 171-176.

416. Rahman S, Bird A, Regina A, et al. (2007) Resistant starch in cereals: exploiting genetic engineering and genetic variation. J Cereal Sci 46, 251-260.

417. Goddard MS, Young G \& Marcus R (1984) The effect of amylose content on insulin and glucose responses to ingested rice. Am J Clin Nutr 39, 388-392.

418. Hallfrisch J \& Behall KM (2000) Mechanisms of the effects of grains on insulin and glucose responses. $J \mathrm{Am}$ Coll Nutr 19, 320S-325S

419. Hawkesford MJ \& Zhao F-J (2007) Strategies for increasing the selenium content of wheat. J Cereal Sci 46, 282-292.

420. Singh BR (1991) Selenium content of wheat as affected by selenate and selenite contained in a Cl- or $\mathrm{SO}_{4}$-based NPK fertilizer. Fertilizer Res 30, 1-7.

421. Soliman MF (1980) Zinc uptake by wheat plants as influenced by nitrogen fertilizers and calcium carbonate. Agric Res Rev 58, 113-121.

422. Fallahi E, Mohtadinia J \& Ali Mahboob S (2005) Effect of consumption of whole bread baked from cultivated wheat with micronutrient fertilizers on blood indices of iron. J Food Agric Environ 3, 39-42.

423. Hanson AD \& Wyse R (1982) Biosynthesis, translocation, and accumulation of betaine in sugar beet and its progenitors in relation to salinity. Plant Physiol $\mathbf{7 0}$ $1191-1198$. 
424. Keles Y \& Öncel I (2002) Response of antioxidative defence system to temperature and water stress combinations in wheat seedlings. Plant Sci 163, 783-790.

425. King JC (2002) Biotechnology: a solution for improving nutrient bioavailability. Int J Vitam Nutr Res 72, 7-12.

426. Cakmak I, Ozkan H, Braun HJ, et al. (2000) Zinc and iron concentrations in seeds of wild, primitive, and modern wheats. Food Nutr Bull 21, 401-403.

427. Ortiz-Monasterio JI, Palacios-Rojas N, Meng E, et al. (2007) Enhancing the mineral and vitamin content of wheat and maize through plant breeding. J Cereal Sci 46, 293-307.

428. Mendoza C, Viteri F, Lonnerdal B, et al. (1998) Effect of genetically modified, low-phytic acid maize on absorption of iron from tortillas. Am J Clin Nutr 68, 1123-1127.

429. Raboy V (2002) Progress in breeding low phytate crops. $J$ Nutr 132, 503S-505S.

430. King RA, Noakes M, Bird AR, et al. (2008) An extruded breakfast cereal made from a high amylose barley cultivar has a low glycemic index and lower plasma insulin response than one made from a standard barley. J Cereal Sci 48, 526-530.

431. Regina A, Bird A, Topping D, et al. (2006) High-amylose wheat generated by RNA interference improves indices of large-bowel health in rats. Proc Natl Acad Sci U S A 103, 3546-3551.

432. Saulnier L, Sado P-E, Branlard G, et al. (2007) Wheat arabinoxylans: exploiting variation in amount and composition to develop enhanced varieties. J Cereal Sci 46, 261-281.

433. Brinch-Pedersen H, Borg S, Tauris B, et al. (2007) Molecular genetic approaches to increasing mineral availability and vitamin content of cereals. J Cereal Sci 46, 308-326.

434. Hammes WP, Brandt MJ, Francis KL, et al. (2005) Microbial ecology of cereal fermentations. Trends Food Sci Technol 16, 4-11.

435. Nout MJR (2009) Rich nutrition from the poorest - cereal fermentations in Africa and Asia. Food Microbiology 26, $685-692$.

436. Napolitano A, Lanzuise S, Ruocco M, et al. (2006) Treatment of cereal products with a tailored preparation of Trichoderma enzymes increases the amount of soluble dietary fiber. J Agric Food Chem 54, 7863-7869.

437. Faulds CB \& Williamson G (1995) Release of ferulic acid from wheat bran by a ferulic acid esterase (FAE-III) from Aspergillus niger. Appl Microbiol Biotechnol 43, $1082-1087$.

438. Wang XK, Geng X, Egashira Y, et al. (2005) Release of ferulic acid from wheat bran by an inducible feruloyl esterase from an intestinal bacterium Lactobacillus acidophilus. Food Sci Technol Res 11, 241-247.

439. Chavan JK \& Kadam SS (1989) Nutritional improvement of cereals by fermentation. Crit Rev Food Sci Nutr 28, $349-400$

440. Gadaga TH, Mutukumira AN, Narvhus JA, et al. (1999) A review of traditional fermented foods and beverages of Zimbabwe. Int J Food Microbiol 53, 1-11.

441. Lioger D, Leenhardt F \& Rémésy C (2006) Intérêt de la fermentation, en milieu très hydraté, des produits céréaliers riches en fibres pour améliorer leur valeur nutritionnelle (Interest of fibre-rich cereal products fermentation in very hydrated environment to improve their nutritional value). Ind Cér 149, 14-22.

442. Poutanen K, Flander L \& Katina K (2009) Sourdough and cereal fermentation in a nutritional perspective. Food Microbiol 26, 693-699.
443. Abd Elmoneim OE, Schiffler B \& Bernhard R (2004) Effect of fermentation on the starch digestibility, resistant starch and some physicochemical properties of sorghum flour. Nahrung/Food 48, 91-94.

444. Eklund-Jonsson C, Sandberg A-S \& Larsson Alminger M (2006) Reduction of phytate content while preserving minerals during whole grain cereal tempe fermentation. $J$ Cereal Sci 44, 154-160.

445. El Hag ME, El Tinay AH \& Yousif NE (2002) Effect of fermentation and dehulling on starch, total polyphenols, phytic acid content and in vitro protein digestibility of pearl millet. Food Chem 77, 193-196.

446. Loponen J, Kanerva P, Zhang C, et al. (2009) Prolamin hydrolysis and pentosan solubilization in germinated-rye sourdoughs determined by chromatographic and immunological methods. J Agric Food Chem 57, 746-753.

447. Mugula JK, Sorhaug T \& Stepaniak L (2003) Proteolytic activities in togwa, a Tanzanian fermented food. Int J Food Microbiol 84, 1-12.

448. Thiele C, Grassl S \& Ganzle M (2004) Gluten hydrolysis and depolymerization during sourdough fermentation. J Agric Food Chem 52, 1307-1314.

449. Wedad HA, El-Tinay AH, Mustafa AI, et al. (2008) Effect of fermentation, malt-pretreatment and cooking on antinutritional factors and protein digestibility of sorghum cultivars. Pak $J$ Nutr 7, 335-341.

450. Lopez HW, Krespine V, Guy C, et al. (2001) Prolonged fermentation of whole wheat sourdough reduces phytate level and increases soluble magnesium. J Agric Food Chem 49, 2657-2662.

451. Lopez HW, Duclos V, Coudray C, et al. (2003) Making bread with sourdough improves mineral bioavailability from reconstituted whole wheat flour in rats. Nutrition $\mathbf{1 9}$, 524-530.

452. Katina K, Liukkonen K-H, Kaukovirta-Norja A, et al. (2007) Fermentation-induced changes in the nutritional value of native or germinated rye. J Cereal Sci 46, 348-355.

453. Winata A \& Lorenz K (1997) Effects of fermentation and baking of whole wheat and whole rye sourdough breads on cereal alkylresorcinols. Cereal Chem 74, 284-287.

454. Katina K, Laitila A, Juvonen R, et al. (2007) Bran fermentation as a means to enhance technological properties and bioactivity of rye. Food Microbiol 24, $175-186$.

455. Moore J, Luther M, Cheng Z, et al. (2009) Effects of baking conditions, dough fermentation, and bran particle size on antioxidant properties of whole-wheat pizza crusts. J Agric Food Chem 57, 832-839.

456. Garcia AL, Otto B, Reich SC, et al. (2007) Arabinoxylan consumption decreases postprandial serum glucose, serum insulin and plasma total ghrelin response in subjects with impaired glucose tolerance. Eur J Clin Nutr 61, 334-341.

457. Lioger D, Fardet A, Foassert P, et al. (2009) Influence of sourdough prefermentation, of steam cooking suppression and of decreased sucrose content during wheat flakes processing on the plasma glucose and insulin responses and satiety of healthy subjects. J Am Coll Nutr 28, 30-36.

458. Liljeberg H \& Bjorck I (1996) Delayed gastric emptying rate as a potential mechanism for lowered glycemia after eating sourdough bread: studies in humans and rats using test products with added organic acids or an organic salt. Am J Clin Nutr 64, 886-893.

459. Liljeberg HG, Lonner CH \& Bjorck IM (1995) Sourdough fermentation or addition of organic acids or corresponding salts to bread improves nutritional properties of starch in healthy humans. J Nutr 125, 1503-1511. 
460. Brennan CS, Blake DE, Ellis PR, et al. (1996) Effects of guar galactomannan on wheat bread microstructure and on the in vitro and in vivo digestibility of starch in bread. J Cereal Sci 24, 151-160.

461. Burton P \& Lightowler HJ (2006) Influence of bread volume on glycaemic response and satiety. Br J Nutr 96, 877-882.

462. Granfeldt Y, Eliasson AC \& Bjorck I (2000) An examination of the possibility of lowering the glycemic index of oat and barley flakes by minimal processing. J Nutr 130, 2207-2214.

463. Antoine C, Lullien-Pellerin V, Abecassis J, et al. (2002) Nutritional interest of the wheat seed aleurone layer. Sci Alim 22, 545-556.

464. Buri RC, von Reding W \& Gavin MH (2004) Description and characterization of wheat aleurone. Cereal Foods World 49, 274-282.

465. Harris PJ, Chavan RR \& Ferguson LR (2005) Production and characterisation of two wheat-bran fractions: an aleurone-rich and a pericarp-rich fraction. Mol Nutr Food Res 49, 536-545.

466. Hemery Y, Rouau X, Lullien-Pellerin V, et al. (2007) Dry processes to develop wheat fractions and products with enhanced nutritional quality. J Cereal Sci 46, 327-347.

467. Fenech M, Noakes M, Clifton P, et al. (1999) Aleurone flour is a rich source of bioavailable folate in humans. J Nutr 129, 1114-1119.

468. Fenech M, Noakes M, Clifton P, et al. (2005) Aleurone flour increases red-cell folate and lowers plasma homocyst(e)ine substantially in man. Br J Nutr 93, 353-360.

469. Cheng BO, Trimble RP, Illman RJ, et al. (1987) Comparative effects of dietary wheat bran and its morphological components (aleurone and pericarp-seed coat) on volatile fatty acid concentrations in the rat. $\mathrm{Br} J$ Nutr 57, 69-76.

470. McIntosh GH, Royle PJ \& Pointing G (2001) Wheat aleurone flour increases cecal $\beta$-glucuronidase activity and butyrate concentration and reduces colon adenoma burden in azoxymethane-treated rats. J Nutr 131, 127-131.

471. Amrein TM, Granicher P, Arrigoni E, et al. (2003) In vitro digestibility and colonic fermentability of aleurone isolated from wheat bran. Lebensm-Wiss Technol Food Sci Technol 36, 451-460.

472. Fardet A, Hoebler C, Baldwin PM, et al. (1998) Involvement of the protein network in the in vitro degradation of starch from spaghetti and lasagne: a microscopic and enzymic study. J Cereal Sci 27, 133-145.

473. Chu FS \& Li GY (1994) Simultaneous occurrence of fumonisin B1 and other mycotoxins in moldy corn collected from the People's Republic of China in regions with high incidences of esophageal cancer. Appl Environ Microbiol 60, 847-852.

474. Rheeder JP, Marasas WFO, Thiel PG, et al. (1992) Fusarium moniliforme and fumonisins in corn in relation to human esophageal cancer in Transkei. Phytopathology 82, 353-357.

475. Lebailly P, Niez E \& Baldi I (2007) Données épidémiologiques sur le lien entre cancers et pesticides (Epidemiological data on the link between cancer and pesticides). Oncologie 9, 361-369.

476. Surget A \& Barron C (2005) Histologie du grain de blé (Histology of the wheat grain). Ind Cér 145, 3-7.

477. Zeisel SH, Mar MH, Howe JC, et al. (2003) Concentrations of choline-containing compounds and betaine in common foods. J Nutr 133, 1302-1307.
478. Poutanen K, Shepherd R, Shewry PR, et al. (2008) Beyond whole grain: The European HEALTHGRAIN project aims at healthier cereal foods. Cereal Foods World 53, 32-35.

479. US Department of Agriculture (2005) USDA National Nutrient Database for Standard Reference, Release 18, Cereal grains and pasta. http://www.nal.usda.gov/fnic/ foodcomp/Data/SR18/reports/sr18page.htm

480. Archer MJ (1972) Relationship between free glutathione content and quality assessment parameters of wheat cultivars (Triticum aestivum L.). J Sci Food Agric 23, 485-491.

481. Shewry PR (2007) Improving the protein content and composition of cereal grain. J Cereal Sci 46, 239-250.

482. Souci SW, Fachmann W \& Kraut H (2008) Food Composition and Nutritional Tables, 7th ed.. Stuttgart, Germany: MedPharm Scientific Publishers.

483. Waggle DH, Lambert MA, Miller GD, et al. (1967) Extensive analyses of flours and millfeeds made from nine different wheat mixes. II. amino acids, minerals, vitamins, and gross energy. Cereal Chem 44, 48-60.

484. Colonna P, Buléon A, Leloup V, et al (1995) Constituants des céréales, des graines, des fruits et de leurs sousproduits (Constituents of grains, seeds, fruits and their by-products). In Nutrition des ruminants domestiques. Ingestionetdigestion(Nutritionofdomesticruminants.Ingestion and digestion), chapter 3 [R Jarrige, Y Ruckebusch and C Demarquilly, et al., editors]. Paris: INRA.

485. Knudsen KEB (1997) Carbohydrate and lignin contents of plant materials used in animal feeding. Anim Feed Sci Tech 67, 319-338.

486. Gebruers K, Dornez E, Boros D, et al. (2008) Variation in the content of dietary fiber and components thereof in wheats in the HEALTHGRAIN Diversity Screen. J Agric Food Chem 56, 9740-9749.

487. Haskå L, Nyman M \& Andersson R (2008) Distribution and characterisation of fructan in wheat milling fractions. J Cereal Sci 48, 768-774.

488. Hernot DC, Boileau TW, Bauer LL, et al. (2008) In vitro digestion characteristics of unprocessed and processed whole grains and their components. J Agric Food Chem 56, $10721-10726$.

489. Nyström L, Paasonen A, Lampi A-M, et al. (2007) Total plant sterols, steryl ferulates and steryl glycosides in milling fractions of wheat and rye. J Cereal Sci $\mathbf{4 5}$, $106-115$.

490. Picolli da Silva L \& de Lourdes Santorio Ciocca M (2005) Total, insoluble and soluble dietary fiber values measured by enzymatic-gravimetric method in cereal grains. J Food Comp Anal 18, 113-120.

491. Ragaee SM, Campbell GL, Scoles GJ, et al. (2001) Studies on rye (Secale cereale L.) lines exhibiting a range of extract viscosities. 1. Composition, molecular weight distribution of water extracts, and biochemical characteristics of purified water-extractable arabinoxylan. J Agric Food Chem 49, 2437-2445.

492. Ward JL, Poutanen K, Gebruers K, et al. (2008) The HEALTHGRAIN Cereal Diversity Screen: concept, results, and prospects. J Agric Food Chem 56, 9699-9709.

493. Abdel-Aal E-SM \& Hucl P (1999) A rapid method for quantifying total anthocyanins in blue aleurone and purple pericarp wheats. Cereal Chem 76, 350-354.

494. Anderson J \& Bridges S (1988) Dietary fiber content of selected foods. Am J Clin Nutr 47, 440-447.

495. Fretzdorff B \& Welge N (2003) Fructan and raffinose contents in cereals and pseudo-cereal grains. Getreide, Mehl und Brot 57, 3-8. 
496. Huynh BL, Palmer L, Mather DE, et al. (2008) Genotypic variation in wheat grain fructan content revealed by a simplified HPLC method. J Cereal Sci 48, 369-378.

497. Huynh BL, Wallwork H, Stangoulis JCR, et al. (2008) Quantitative trait loci for grain fructan concentration in wheat (Triticum aestivum L.). Theor Appl Genet 117, $701-709$.

498. Henry RJ (1987) Pentosan and $(1 \rightarrow 3),(1 \rightarrow 4)$ - $\beta$-glucan concentrations in endosperm and wholegrain of wheat, barley, oats and rye. J Cereal Sci 6, 253-258.

499. Lempereur I, Rouau X \& Abecassis J (1997) Genetic and agronomic variation in arabinoxylan and ferulic acid contents of durum wheat (Triticum durum L.) grain and its milling fractions. J Cereal Sci 25, 103-110.

500. Genç H, Özdemir M \& Demirbas A (2001) Analysis of mixed-linked $(1 \rightarrow 3)$, $(1 \rightarrow 4)-\beta$-D-glucans in cereal grains from Turkey. Food Chem 73, 221-224.

501. Hemery Y, Lullien-Pellerin V, Rouau X, et al. (2009) Biochemical markers: efficient tools for the assessment of wheat grain tissue proportions in milling fractions. J Cereal Sci 49, 55-64.

502. House WA \& Welch RM (1987) Bioavailability to rats of iron in 6 varieties of wheat-grain intrinsically labeled with radioiron. J Nutr 117, 476-480.

503. Lopez HW, Krespine V, Lemaire A, et al. (2003) Wheat variety has a major influence on mineral bioavailability; studies in rats. J Cereal Sci 37, 257-266.

504. O’Dell BL, Burpo CE \& Savage JE (1972) Evaluation of zinc availability in foodstuffs of plant and animal origin. J Nutr 102, 653-660.

505. Tabekhia MM \& Donnelly BJ (1982) Phytic acid in durum-wheat and its milled products. Cereal Chem 59, $105-107$.

506. Tariq M, Talat M, Asia L, et al. (2007) Influence of processing and cooking methodologies for reduction of phytic acid content in wheat (Triticum aestivum) varieties. J Food Process Preserv 31, 583-594.

507. Davis KR, Peters LJ, Cain RF, et al. (1984) Evaluation of the nutrient composition of wheat. III. Minerals. Cereal Foods World 29, 246-248.

508. Frossard E, Bucher M, Mächler F, et al. (2000) Potential for increasing the content and bioavailability of $\mathrm{Fe}, \mathrm{Zn}$ and $\mathrm{Ca}$ in plants for human nutrition. J Sci Food Agric 80, $861-879$.

509. Lorenz K \& Loewe R (1977) Mineral composition of U.S. and Canadian wheats and wheat blends. J Agric Food Chem 25, 806-809.

510. Monasterio I \& Graham RD (2000) Breeding for trace minerals in wheat. Food Nutr Bull 21, 392-396.

511. Tang J, Zou C, He Z, et al. (2008) Mineral element distributions in milling fractions of Chinese wheats. J Cereal Sci 48, 821-828.

512. Zook EG, Greene FE \& Morris ER (1970) Nutrient composition of selected wheats and wheat products. VI. Distribution of manganese, copper, nickel, zinc, magnesium, lead, tin, cadmium, chromium, and selenium as determined by atomic absorption spectroscopy and colorimetry. Cereal Chem 47, 720-731.

513. Welch RM \& Graham RD (2000) A new paradigm for world agriculture: productive, sustainable, nutritious, healthful food systems. Food Nutr Bull 21, 361-366.

514. Fan MS, Zhao FJ, Poulton PR, et al. (2008) Historical changes in the concentrations of selenium in soil and wheat grain from the Broadbalk experiment over the last 160 years. Sci Total Environ 389, 532-538.

515. Zhao F, McGrath S, Gray C, et al. (2007) Selenium concentrations in UK wheat and biofortification strategies.
Comp Biochem Physiol A Mol Integr Physiol 146, Suppl. 1, S246.

516. Batifoulier F, Verny M-A, Chanliaud E, et al. (2005) Effect of different breadmaking methods on thiamine, riboflavin and pyridoxine contents of wheat bread. J Cereal Sci $\mathbf{4 2}$, $101-108$.

517. Davis KR, Cain RF, Peters LJ, et al. (1981) Evaluation of the nutrient composition of wheat. 2. Proximate analysis, thiamin, riboflavin, niacin, and pyridoxine. Cereal Chem 58, 116-120.

518. Davis KR, Peters LJ \& Letourneau D (1984) Variability of the vitamin content in wheat. Cereal Foods World 29, 364-370.

519. Ranhotra G, Gelroth J, Novak F, et al. (1985) Bioavailability for rats of thiamin in whole wheat and thiaminrestored white bread. J Nutr 115, 601-606.

520. Gujska E \& Kuncewicz A (2005) Determination of folate in some cereals and commercial cereal-grain products consumed in Poland using trienzyme extraction and highperformance liquid chromatography methods. Eur Food Res Technol 221, 208-213.

521. Perloff BP \& Butrum RR (1977) Folacin in selected foods. $J$ Am Diet Assoc 70, 161-172.

522. Piironen V, Edelmann M, Kariluoto S, et al. (2008) Folate in wheat genotypes in the HEALTHGRAIN Diversity Screen. J Agric Food Chem 56, 9726-9731.

523. Lampi A-M, Nurmi T, Ollilainen V, et al. (2008) Tocopherols and tocotrienols in wheat genotypes in the HEALTHGRAIN Diversity Screen. J Agric Food Chem 56, 9716-9721.

524. Nielsen MM \& Hansen A (2008) Stability of vitamin E in wheat flour and whole wheat flour during storage. Cereal Chem 85, 716-720.

525. Nielsen MM \& Hansen A (2008) Rapid high-performance liquid chromatography determination of tocopherols and tocotrienols in cereals. Cereal Chem 85, 248-251.

526. Panfili G, Fratianni A \& Irano M (2003) Normal phase high-performance liquid chromatography method for the determination of tocopherols and tocotrienols in cereals. J Agric Food Chem 51, 3940-3944.

527. Moore J, Hao Z, Zhou K, et al. (2005) Carotenoid, tocopherol, phenolic acid, and antioxidant properties of Maryland-grown soft wheat. J Agric Food Chem 53, 6649-6657.

528. Konopka I, Kozirok W \& Rotkiewicz D (2004) Lipids and carotenoids of wheat grain and flour and attempt of correlating them with digital image analysis of kernel surface and cross-sections. Food Res Int 37, 429-438.

529. Leenhardt F, Lyan B, Rock E, et al. (2006) Genetic variability of carotenoid concentration, and lipoxygenase and peroxidase activities among cultivated wheat species and bread wheat varieties. Eur J Agron 25, 170-176.

530. Panfili G, Fratianni A \& Irano M (2004) Improved normalphase high-performance liquid chromatography procedure for the determination of carotenoids in cereals. J Agric Food Chem 52, 6373-6377.

531. Roose M, Kahl J \& Ploeger A (2009) Influence of the farming system on the xanthophyll content of soft and hard wheat. J Agric Food Chem 57, 182-188.

532. Adom KK \& Liu RH (2002) Antioxidant activity of grains. J Agric Food Chem 50, 6182-6187.

533. Barron C, Surget A \& Rouau X (2007) Relative amounts of tissues in mature wheat (Triticum aestivum L.) grain and their carbohydrate and phenolic acid composition. J Cereal Sci 45, 88-96.

534. Lempereur I, Surget A \& Rouau X (1998) Variability in dehydrodiferulic acid composition of durum wheat 
(Triticum durum Desf.) and distribution in milling fractions. J Cereal Sci 28, 251-258.

535. Mpofu A, Sapirstein HD \& Beta T (2006) Genotype and environmental variation in phenolic content, phenolic acid composition, and antioxidant activity of hard spring wheat. J Agric Food Chem 54, 1265-1270.

536. Abdel-Aal ESM \& Hucl P (2003) Composition and stability of anthocyanins in blue-grained wheat. J Agric Food Chem 51, 2174-2180.

537. Abdel-Aal E-SM, Abou-Arab AA, Gamel TH, et al. (2008) Fractionation of blue wheat anthocyanin compounds and their contribution to antioxidant properties. J Agric Food Chem 56, 11171-11177.

538. Liggins J, Mulligan A, Runswick S, et al. (2002) Daidzein and genistein content of cereals. Eur J Clin Nutr 56, 961-966.

539. Dinelli G, Marotti I, Bosi S, et al. (2007) Lignan profile in seeds of modern and old Italian soft wheat (Triticum aestivum L.) cultivars as revealed by CE-MS analyses. Electrophoresis 28, 4212-4219.

540. Milder IEJ, Feskens EJM, Arts ICW, et al. (2005) Intake of the plant lignans secoisolariciresinol, matairesinol, lariciresinol, and pinoresinol in Dutch men and women. $J$ Nutr 135, 1202-1207.

541. Andersson AAM, Kamal-Eldin A, Fraś A, et al. (2008) Alkylresorcinols in wheat varieties in the HEALTHGRAIN Diversity Screen. J Agric Food Chem 56, 9722-9725.

542. US Department of Agriculture ARS, Nutrient Data Laboratory, Patterson KY \& Bhagwat SA (2008) et al. USDA database for the choline content of common foods, release 2. http://www.nal.usda.gov/fnic/foodcomp/Data/ Choline/Choln02.pdf (accessed 2008).

543. Hakala P, Lampi A-M, Ollilainen V, et al. (2002) Steryl phenolic acid esters in cereals and their milling fractions. J Agric Food Chem 50, 5300-5307.

544. Iafelice G, Verardo V, Marconi E, et al. (2009) Characterization of total, free and esterified phytosterols in tetraploid and hexaploid wheats. J Agric Food Chem 57, 2267-2273.

545. Nurmi T, Nyström L, Edelmann M, et al. (2008) Phytosterols in wheat genotypes in the HEALTHGRAIN Diversity Screen. J Agric Food Chem 56, 9710-9715.

546. Piironen V, Toivo J \& Lampi AM (2002) Plant sterols in cereals and cereal products. Cereal Chem 79, 148-154.

547. Irmak S \& Dunford NT (2005) Policosanol contents and compositions of wheat varieties. J Agric Food Chem 53, 5583-5586.

548. Trautwein EA (2001) $n$-3 Fatty acids - physiological and technical aspects for their use in food. Eur J Lipid Sci Technol 103, 45-55.

549. Every D, Morrison SC, Simmons LD, et al. (2006) Distribution of glutathione in millstreams and relationships to chemical and baking properties of flour. Cereal Chem 83, 57-61.

550. Fraser JR \& Holmes DC (1959) Proximate analysis of wheat flour carbohydrates. IV. - analysis of wholemeal flour and some of its fractions. J Sci Food Agric 10, 506-512.

551. Saunders RM \& Walker HG (1969) Sugars of wheat bran. Cereal Chem 46, 85.

552. Chen H, Haack V, Janecky C, et al. (1998) Mechanisms by which wheat bran and oat bran increase stool weight in humans. Am J Clin Nutr 68, 711-719.

553. Lehrfeld J \& Wu YV (1991) Distribution of phytic acid in milled fractions of Scout-66 hard red winter-wheat. J A gric Food Chem 39, 1820-1824.
554. Maes C, Vangeneugden B \& Delcour JA (2004) Relative activity of two endoxylanases towards water-unextractable arabinoxylans in wheat bran. J Cereal Sci 39, 181-186.

555. Esposito F, Arlotti G, Bonifati AM, et al. (2005) Antioxidant activity and dietary fibre in durum wheat bran by-products. Food Res Int 38, 1167-1173.

556. Gordon DT \& Chao LS (1984) Relationship of components in wheat bran and spinach to iron bioavailability in the anemic rat. J Nutr 114, 526-535.

557. Morris ER \& Ellis R (1980) Bioavailability to rats of iron and zinc in wheat bran - response to low-phytate bran and effect of the phytate-zinc molar ratio. $J$ Nutr 110, 2000-2010.

558. Anderson NE \& Clydesdale FM (1980) An analysis of the dietary fiber content of a standard wheat bran. J Food Sci 45, 336-340.

559. Bagheri SM \& Gueguen L (1982) Bioavailability to rats of calcium, magnesium, phosphorus and zinc in wheat bran diets containing equal amounts of these minerals. Nutr Rep Int 25, 583-589.

560. Falcao-e-Cunha L, Peres H, Freire JPB, et al. (2004) Effects of alfalfa, wheat bran or beet pulp, with or without sunflower oil, on caecal fermentation and on digestibility in the rabbit. Anim Feed Sci Tech 117, 131-149.

561. Heller S, Hackler L, Rivers J, et al. (1980) Dietary fiber: the effect of particle size of wheat bran on colonic function in young adult men. Am J Clin Nutr 33, 1734-1744.

562. Maes C \& Delcour JA (2002) Structural characterisation of water-extractable and water-unextractable arabinoxylans in wheat bran. J Cereal Sci 35, 315-326.

563. Dornez E, Gebruers K, Wiame S, et al. (2006) Insight into the distribution of arabinoxylans, endoxylanases, and endoxylanase inhibitors in industrial wheat roller mill streams. J Agric Food Chem 54, 8521-8529.

564. Camire AL \& Clydesdale FM (1982) Analysis of phytic acid in foods by HPLC. J Food Sci 47, 575-578.

565. Fretzdorff B (1989) Phytic acid in wheat bran and germ products - how to remove phytic acid from these products. Z Lebens Unters Forsch 189, 110-112.

566. Jenab M \& Thompson LU (2000) Phytic acid in wheat bran affects colon morphology, cell differentiation and apoptosis. Carcinogenesis 21, 1547-1552.

567. Liu ZH, Wang HY, Wang XE, et al. (2008) Effect of wheat pearling on flour phytase activity, phytic acid, iron, and zinc content. LWT Food Sci Technol 41, 521-527.

568. Bagheri S \& Guéguen L (1985) Effect of wheat bran and pectin on the absorption and retention of phosphorus, calcium, magnesium and zinc by the growing-pig. Reprod Nutr Dev 25, 705-716

569. Shils ME, Olson JA \& Shike M (editors) (1994) Modern Nutrition in Health and Disease, 8th ed. Philadelphia, PA: Lea and Febiger.

570. Mullin WJ \& Jui PY (1986) Folate content of bran from different wheat classes. Cereal Chem 63, 516-518.

571. Zhou K, Su L \& Yu LL (2004) Phytochemicals and antioxidant properties in wheat bran. J Agric Food Chem 52, 6108-6114.

572. Zhou KQ, Yin JJ \& Yu LL (2005) Phenolic acid, tocopherol and carotenoid compositions, and antioxidant functions of hard red winter wheat bran. J Agric Food Chem 53, 3916-3922.

573. Robertson JA, Faulds CB, Smith AC, et al. (2008) Peroxidase-mediated oxidative cross-linking and its potential to modify mechanical properties in water-soluble polysaccharide extracts and cereal grain residues. J Agric Food Chem 56, 1720-1726. 
574. Irmak S, Jonnala RS \& MacRitchie F (2008) Effect of genetic variation on phenolic acid and policosanol contents of Pegaso wheat lines. J Cereal Sci 48, 20-26.

575. Kim KH, Tsao R, Yang R, et al. (2006) Phenolic acid profiles and antioxidant activities of wheat bran extracts and the effect of hydrolysis conditions. Food Chem 95, $466-473$.

576. Siebenhandl S, Grausgruber H, Pellegrini N, et al. (2007) Phytochemical profile of main antioxidants in different fractions of purple and blue wheat, and black barley. J Agric Food Chem 55, 8541-8547.

577. Apak R, Güçlü K, Ozyürek M, et al. (2005) Total antioxidant capacity assay of human serum using copper(II)-neocuproine as chromogenic oxidant: the CUPRAC method. Free Radic Res 39, 949-961.

578. Moore J, Liu JG, Zhou KQ, et al. (2006) Effects of genotype and environment on the antioxidant properties of hard winter wheat bran. J Agric Food Chem 54, 5313-5322.

579. Zhou K \& Yu L (2004) Antioxidant properties of bran extracts from Trego wheat grown at different locations. J Agric Food Chem 52, 1112-1117.

580. Iqbal S, Bhanger MI \& Anwar F (2007) Antioxidant properties and components of bran extracts from selected wheat varieties commercially available in Pakistan. LWT Food Sci Technol 40, 361-367.

581. Smeds AI, Eklund PC, Sjoholm RE, et al. (2007) Quantification of a broad spectrum of lignans in cereals, oilseeds, and nuts. J Agric Food Chem 55, 1337-1346.

582. Kulawinek M, Jaromin A, Kozubek A, et al. (2008) Alkylresorcinols in selected Polish rye and wheat cereals and whole-grain cereal products. J Agric Food Chem 56, 7236-7242.

583. Graham SF, Hollis JH, Migaud M, et al. (2009) Analysis of betaine and choline contents of aleurone, bran, and flour fractions of wheat (Triticum aestivum L.) using ${ }^{1} \mathrm{H}$ nuclear magnetic resonance (NMR) spectroscopy. J Agric Food Chem 57, 1948-1951.

584. Slow S, Donaggio M, Cressey PJ, et al. (2005) The betaine content of New Zealand foods and estimated intake in the New Zealand diet. J Food Comp Anal 18, 473-485.

585. Nyström L, Lampi AM, Rita H, et al. (2007) Effects of processing on availability of total plant sterols, steryl ferulates and steryl glycosides from wheat and rye bran. J Agric Food Chem 55, 9059-9065.

586. Irmak S, Dunford NT \& Milligan J (2006) Policosanol contents of beeswax, sugar cane and wheat extracts. Food Chem 95, 312-318.

587. Moruzzi G, Viviani R, Sechi AM, et al. (1969) Studies on compounds and individual lipids of wheat germ. J Food Sci 34, 581-584.

588. Dubois M, Geddes WF \& Smith F (1960) The carbohydrates of the Gramineae. X. A quantitative study of the carbohydrates of wheat germ. Cereal Chem 37, $557-567$.

589. Garcia WJ, Gardner HW, Cavins JF, et al. (1972) Composition of air-classified defatted corn and wheat germ flours. Cereal Chem 49, 499-507.

590. Linko P, Cheng Y-Y \& Milner M (1960) Changes in the soluble carbohydrates during browning of wheat embryos. Cereal Chem 37, 548-556.

591. Leenhardt F, Fardet A, Lyan B, et al. (2008) Wheat germ supplementation of a low vitamin $\mathrm{E}$ diet in rats affords effective antioxidant protection in tissues. $\mathrm{J} \mathrm{Am} \mathrm{Coll} \mathrm{Nutr}$ 27, 222-228.

592. Shurpalekar SR \& Rao PH (1977) Wheat germ. Adv Food Res 23, 187-304.
593. Bilgicli N \& Ibanoglu S (2007) Effect of wheat germ and wheat bran on the fermentation activity, phytic acid content and colour of tarhana, a wheat flour-yoghurt mixture. J Food Eng 78, 681-686.

594. Garcia WJ, Inglett GE \& Blessin CW (1972) Mineral constituents in corn and wheat-germ by atomic-absorption spectroscopy. Cereal Chem 49, 158-167.

595. Zhu KX, Zhou HM \& Qian HF (2006) Comparative study of chemical composition and physicochemical properties of defatted wheat germ flour and its protein isolate. J Food Biochem 30, 329-341.

596. Dodin S, Lemay A, Jacques H, et al. (2005) The effects of flaxseed dietary supplement on lipid profile, bone mineral density, and symptoms in menopausal women: a randomized, double-blind, wheat germ placebo-controlled clinical trial. J Clin Endocrinol Metab 90, 1390-1397.

597. Ostlund RE Jr, Racette SB \& Stenson WF (2003) Inhibition of cholesterol absorption by phytosterol-replete wheat germ compared with phytosterol-depleted wheat germ. Am J Clin Nutr 77, 1385-1389.

598. Mühlum A, Ingwersen M, Schünemann C, et al. (1989) Precaecal and postileal digestion of sucrose, lactose, stachyose and raffinose. Adv Anim Physiol Anim Nutr 19, 31-43.

599. Van Dokkum W, Pikaar NA \& Thissen JT (1983) Physiological effects of fibre-rich types of bread. 2 . Dietary fibre from bread: digestibility by the intestinal microflora and water-holding capacity in the colon of human subjects. Br J Nutr 50, 61-74.

600. McCance RA \& Widdowson EM (1935) Phytin in human nutrition. Biochem J 29, 2694-2699.

601. Sakamoto K, Vucenik I \& Shamsuddin AM (1993) $\left[{ }^{3} \mathrm{H}\right]$ Phytic acid (inositol hexaphosphate) is absorbed and distributed to various tissues in rats. J Nutr 123, 713-720.

602. McCance RA \& Widdowson EM (1942) Mineral metabolism of healthy adults on white and brown bread dietaries. J Physiol 101, 44-85.

603. Institute of Medicine (1997) Dietary Reference Intakes for Calcium, Phosphorus, Magnesium, Vitamin D, and Fluoride. Washington, DC: National Academy Press.

604. Walti MK, Zimmermann MB, Walczyk T, et al. (2003) Measurement of magnesium absorption and retention in type 2 diabetic patients with the use of stable isotopes. Am J Clin Nutr 78, 448-453.

605. Sandstrom B, Arvidsson B, Cederblad A, et al. (1980) Zinc absorption from composite meals. 1. The significance of wheat extraction rate, zinc, calcium, and protein-content in meals based on bread. Am J Clin Nutr 33, 739-745.

606. Sundkvist G, Dahlin LB, Nilsson H, et al. (2000) Sorbitol and myo-inositol levels and morphology of sural nerve in relation to peripheral nerve function and clinical neuropathy in men with diabetic, impaired, and normal glucose tolerance. Diabet Med 17, 259-268.

607. Saha PR, Weaver CM \& Mason AC (1994) Mineral bioavailability in rats from intrinsically labeled whole wheat-flour of various phytate levels. J Agric Food Chem 42, 2531-2535.

608. Fox TE, Fairweather-Tait SJ, Eagles J, et al. (1994) Assessment of zinc bioavailability - studies in rats on zinc absorption from wheat using radio-isotopes and stableisotopes. Br J Nutr 71, 95-101.

609. Welch RM, House WA, Ortiz-Monasterio I, et al. (2005) Potential for improving bioavailable zinc in wheat grain (Triticum species) through plant breeding. J Agric Food Chem 53, 2176-2180. 
610. Ahmed A, Anjum F, Ur Rehman S, et al. (2008) Bioavailability of calcium, iron and zinc fortified whole wheat flour chapatti. Plant Foods Hum Nutr 63, 7-13.

611. Johnson PE \& Lykken GI (1988) Copper-65 absorption by men fed intrinsically and extrinsically labeled whole wheat bread. J Agric Food Chem 36, 537-540.

612. Mutanen M, Koivistoinen P, Morris VC, et al. (2007) Relative nutritional availability to rats of selenium in Finnish spring wheat (Triticum aestivum L.) fertilized or sprayed with sodium selenate and in an American winter bread wheat naturally high in Se. Br J Nutr 57, 319-329.

613. Alexander AR, Whanger PD \& Miller LT (1983) Bioavailability to rats of selenium in various tuna and wheat products. J Nutr 113, 196-204.

614. Weaver CM, Heaney RP, Martin BR, et al. (1991) Human calcium absorption from whole-wheat products. $J$ Nutr 121, 1769-1775.

615. Zempleni J, Galloway J \& McCormick D (1996) Pharmacokinetics of orally and intravenously administered riboflavin in healthy humans. Am J Clin Nutr 63, 54-66.

616. Tarr J, Tamura T \& Stokstad E (1981) Availability of vitamin $\mathrm{B}_{6}$ and pantothenate in an average American diet in man. Am J Clin Nutr 34, 1328-1337.

617. Kayden H \& Traber M (1993) Absorption, lipoprotein transport, and regulation of plasma concentrations of vitamin $\mathrm{E}$ in humans. J Lipid Res 34, 343-358.

618. Andreasen MF, Kroon PA, Williamson G, et al. (2001) Esterase activity able to hydrolyze dietary antioxidant hydroxycinnamates is distributed along the intestine of mammals. J Agric Food Chem 49, 5679-5684.

619. Ross AB, Shepherd MJ, Knudsen KEB, et al. (2003) Absorption of dietary alkylresorcinols in ileal-cannulated pigs and rats. Br J Nutr 90, 787-794.

620. Nissinen M, Gylling H, Vuoristo M, et al. (2002) Micellar distribution of cholesterol and phytosterols after duodenal plant stanol ester infusion. Am J Physiol Gastrointest Liver Physiol 282, G1009-G1015.

621. Nestler JE, Jakubowicz DJ, Reamer P, et al. (1999) Ovulatory and metabolic effects of D-chiro-inositol in the polycystic ovary syndrome. $N$ Engl J Med 340, 1314-1320.

622. Campbell WW, Haub MD, Fluckey JD, et al. (2004) Pinitol supplementation does not affect insulin-mediated glucose metabolism and muscle insulin receptor content and phosphorylation in older humans. J Nutr 134, 2998-3003.

623. Nyman M, Asp N-G, Cummings J, et al. (1986) Fermentation of dietary fibre in the intestinal tract: comparison between man and rat. Br J Nutr 55, 487-496.

624. Kahlon TS, Chow FI, Hoefer JL, et al. (2001) Effect of wheat bran fiber and bran particle size on fat and fiber digestibility and gastrointestinal tract measurements in the rat. Cereal Chem 78, 481-484.

625. Hansen I, Knudsen KEB \& Eggum BO (2007) Gastrointestinal implications in the rat of wheat bran, oat bran and pea fibre. Br J Nutr 68, 451-462.

626. Ehle FR, Jeraci JL, Robertson JB, et al. (1982) The influence of dietary fiber on digestibility, rate of passage and gastrointestinal fermentation in pigs. J Anim Sci 55, $1071-1081$.

627. Robertson JA, Murison SD \& Chesson A (1992) Particlesize distribution and solubility of dietary fiber in swedebran (Brassica napus) based and wheat-bran-based diets during gastrointestinal transit in the pig. J Sci Food Agric 58, 197-205.
628. Nyman M \& Asp N-G (1985) Dietary fibre fermentation in the rat intestinal tract: effect of adaptation period, protein and fibre levels, and particle size. Br J Nutr 54, 635-643.

629. Sandberg AS \& Andersson H (1988) Effect of dietary phytase on the digestion of phytate in the stomach and small intestine of humans. J Nutr 118, 469-473.

630. Sandberg A-S, Andersson H, Carlsson N-G, et al. (1987) Degradation products of bran phytate formed during digestion in the human small intestine: effect of extrusion cooking on digestibility. J Nutr 117, 2061-2065.

631. Brune M, Rossander-Hulten L, Hallberg L, et al. (1992) Iron absorption from bread in humans: inhibiting effects of cereal fiber, phytate and inositol phosphates with different numbers of phosphate groups. J Nutr 122, 442-449.

632. Reddy M \& Cook J (1991) Assessment of dietary determinants of nonheme-iron absorption in humans and rats. Am J Clin Nutr 54, 723-728.

633. Reeves PG, Gregoire BR, Garvin DF, et al. (2007) Determination of selenium bioavailability from wheat mill fractions in rats by using the slope-ratio assay and a modified Torula yeast-based diet. J Agric Food Chem $\mathbf{5 5}$, 516-522.

634. Carter EGA \& Carpenter KJ (1982) The bioavailability for humans of bound niacin from wheat bran. Am J Clin Nutr 36, 855-861

635. Kies C, Kan S \& Fox HM (1984) Vitamin B 6 availability from wheat, rice and corn brans for humans. Nutr Rep Int 30, 483-491.

636. Kahlon TS, Chow FI, Hoefer JL, et al. (1986) Bioavailability of vitamin A and vitamin $\mathrm{E}$ as influenced by wheat bran and bran particle size. Cereal Chem 63, 490-493.

637. Andreasen MF, Kroon PA, Williamson G, et al. (2001) Intestinal release and uptake of phenolic antioxidant diferulic acids. Free Radic Biol Med 31, 304-314.

638. Connor WE (2000) Importance of $n$-3 fatty acids in health and disease. Am J Clin Nutr 71, 171S-175S.

639. Brouwer IA, Katan MB \& Zock PL (2004) Dietary $\alpha$-linolenic acid is associated with reduced risk of fatal coronary heart disease, but increased prostate cancer risk: a meta-analysis. J Nutr 134, 919-922.

640. Hu FB, Stampfer MJ, Manson JE, et al. (1999) Dietary intake of $\alpha$-linolenic acid and risk of fatal ischemic heart disease among women. Am J Clin Nutr 69, 890-897.

641. Kang JX \& Leaf A (1996) Antiarrhythmic effects of polyunsaturated fatty acids: recent studies. Circulation 94, 1774-1780.

642. Edwards R, Peet M, Shay J, et al. (1998) Omega-3 polyunsaturated fatty acid levels in the diet and in red blood cell membranes of depressed patients. J Affect Disord 48, 149-155.

643. Yehuda S, Rabinovitz S \& Mostofsky DI (2005) Mixture of essential fatty acids lowers test anxiety. Nutr Neurosci $\mathbf{8}$ 265-267.

644. Djousse L, Folsom AR, Province MA, et al. (2003) Dietary linolenic acid and carotid atherosclerosis: the National Heart, Lung, and Blood Institute Family Heart Study. Am J Clin Nutr 77, 819-825.

645. Narisawa T, Fukaura Y, Yazawa K, et al. (1994) Colon cancer prevention with a small amount of dietary perilla oil high in $\alpha$-linolenic acid in an animal model. Cancer 73, 2069-2075.

646. Klein V, Chajes V, Germain E, et al. (2000) Low $\alpha$-linolenic acid content of adipose breast tissue is associated with an increased risk of breast cancer. Eur $J$ Cancer 36, 335-340. 
647. Hwang DH, Boudreau M \& Chanmugam P (1988) Dietary linolenic acid and longer-chain $n$ - 3 fatty acids: comparison of effects on arachidonic acid metabolism in rats. $J$ Nutr 118, 427-437.

648. Chapkin RS, McMurray DN, Davidson LA, et al. (2008) Bioactive dietary long-chain fatty acids: emerging mechanisms of action. Br J Nutr 100, 1152-1157.

649. Enke U, Seyfarth L, Schleussner E, et al. (2008) Impact of PUFA on early immune and fetal development. Br J Nutr 100, 1158-1168.

650. Townsend DM, Tew KD \& Tapiero H (2003) The importance of glutathione in human disease. Biomed Pharmacother 57, 145-155.

651. Higashi T, Tateishi N, Naruse A, et al. (1977) A novel physiological role of liver glutathione as a reservoir of L-cysteine. J Biochem 82, 117-124.

652. Bilzer M \& Lauterburg BH (1991) Effects of hypochlorous acid and chloramines on vascular resistance, cell integrity, and biliary glutathione disulfide in the perfused rat-liver modulation by glutathione. J Hepatol 13, 84-89.

653. Troen AM, Chao W-H, Crivello NA, et al. (2008) Cognitive impairment in folate-deficient rats corresponds to depleted brain phosphatidylcholine and is prevented by dietary methionine without lowering plasma homocysteine. J Nutr 138, 2502-2509.

654. Essien FB \& Wannberg SL (1993) Methionine but not folinic acid or vitamin B-12 alters the frequency of neural tube defects in axd mutant mice. J Nutr 123, 27-34.

655. Caylak E, Aytekin M \& Halifeoglu I (2008) Antioxidant effects of methionine, $\alpha$-lipoic acid, $N$-acetylcysteine and homocysteine on lead-induced oxidative stress to erythrocytes in rats. Exp Toxicol Pathol 60, 289-294.

656. Khumalo N, Dawber R \& Ferguson D (2005) Apparent fragility of African hair is unrelated to the cystine-rich protein distribution: a cytochemical electron microscopic study. Exp Dermatol 14, 311-314.

657. Sass J, Skladal D, Zelger B, et al. (2004) Trichothiodystrophy: quantification of cysteine in human hair and nails by application of sodium azide-dependent oxidation to cysteic acid. Arch Dermatol Res 296, 188-191.

658. Droge W \& Holm E (1997) Role of cysteine and glutathione in HIV infection and other diseases associated with muscle wasting and immunological dysfunction. FASEB J 11, 1077-1089.

659. Netto LES, de Oliveira MA, Monteiro G, et al. (2007) Reactive cysteine in proteins: protein folding, antioxidant defense, redox signaling and more. Comp Biochem Physiol C Toxicol Pharmacol 146, 180-193.

660. Marlett JA, McBurney MI \& Slavin JL (2002) Position of the American Dietetic Association: health implications of dietary fiber. J Am Diet Assoc 102, 993-1000.

661. Tucker LA \& Thomas KS (2009) Increasing total fiber intake reduces risk of weight and fat gains in women. J Nutr 139, 576-581.

662. Salmeron J, Ascherio A, Rimm EB, et al. (1997) Dietary fiber, glycemic load, and risk of non-insulin-dependent diabetes mellitus in women. Diabetes Care 277, 472-477.

663. Slavin JL, Jacobs D, Marquart L, et al. (2001) The role of whole grains in disease prevention. J Am Diet Assoc 101, 780-785.

664. Glei M, Hofmann T, Kuster K, et al. (2006) Both wheat (Triticum aestivum) bran arabinoxylans and gut floramediated fermentation products protect human colon cells from genotoxic activities of 4-hydroxynonenal and hydrogen peroxide. J Agric Food Chem 54, 2088-2095.
665. Eastwood M \& Mowbray L (1976) The binding of the components of mixed micelle to dietary fiber. Am J Clin Nutr 29, $1461-1467$.

666. Pomare EW \& Heaton KW (1973) Alteration of bile salt metabolism by dietary fibre (bran). Gut 14, 826.

667. Eastwood MA (1975) Vegetable dietary fiber - potent pith. J R Soc Health 95, 188-190.

668. Kaur N \& Gupta AK (2002) Applications of inulin and oligofructose in health and nutrition. J Biosci 27, 703-714.

669. Roberfroid MB \& Delzenne NM (1998) Dietary fructans. Annu Rev Nutr 18, 117-143.

670. Rozan P, Nejdi A, Hidalgo S, et al. (2008) Effects of lifelong intervention with an oligofructose-enriched inulin in rats on general health and lifespan. Br J Nutr 100, 1192-1199.

671. Gibson GR, Beatty ER, Wang X, et al. (1995) Selective stimulation of bifidobacteria in the human colon by oligofructose and inulin. Gastroenterology 108, 975-982.

672. Femia AP, Luceri C, Dolara P, et al. (2002) Antitumorigenic activity of the prebiotic inulin enriched with oligofructose in combination with the probiotics Lactobacillus rhamnosus and Bifidobacterium lactis on azoxymethane-induced colon carcinogenesis in rats. Carcinogenesis 23, 1953-1960.

673. Archer SY, Meng S, Shei A, et al. (1998) p21WAF1 is required for butyrate-mediated growth inhibition of human colon cancer cells. Proc Natl Acad Sci U S A 95, 6791-6796.

674. Avivi-Green C, Polak-Charcon S, Madar Z, et al. (2002) Different molecular events account for butyrate-induced apoptosis in two human colon cancer cell lines. J Nutr 132, 1812-1818.

675. Brighenti F, Casiraghi MC, Canzi E, et al. (1999) Effect of consumption of a ready-to-eat breakfast cereal containing inulin on the intestinal milieu and blood lipids in healthy male volunteers. Eur J Clin Nutr 53, 726-733.

676. Williams CM (1999) Effects of inulin on lipid parameters in humans. J Nutr 129, 1471S-1473S.

677. Beylot M (2005) Effects of inulin-type fructans on lipid metabolism in man and in animal models. Br J Nutr 93, S163-S168.

678. Pai R, Tarnawski AS \& Tran T (2004) Deoxycholic acid activates $\beta$-catenin signaling pathway and increases colon cell cancer growth and invasiveness. Mol Biol Cell 15, 2156-2163.

679. McMillan L, Butcher S, Wallis Y, et al. (2000) Bile acids reduce the apoptosis-inducing effects of sodium butyrate on human colon adenoma (AA/C1) cells: implications for colon carcinogenesis. Biochem Biophys Res Commun 273, 45-49.

680. Braaten JT, Wood PJ, Scott FW, et al. (1991) Oat gum lowers glucose and insulin after an oral glucose load. Am J Clin Nutr 53, 1425-1430.

681. Ostman E, Rossi E, Larsson H, et al. (2006) Glucose and insulin responses in healthy men to barley bread with different levels of $(1 \rightarrow 3 ; 1 \rightarrow 4)$ - $\beta$-glucans; predictions using fluidity measurements of in vitro enzyme digests. J Cereal Sci 43, 230-235.

682. Tappy L, Gugolz E \& Wursch P (1996) Effects of breakfast cereals containing various amounts of $\beta$-glucan fibers on plasma glucose and insulin responses in NIDDM subjects. Diabetes Care 19, 831-834.

683. Maki KC, Shinnick F, Seeley MA, et al. (2003) Food products containing free tall oil-based phytosterols and oat $\beta$-glucan lower serum total and LDL cholesterol in hypercholesterolemic adults. J Nutr 133, 808-813. 
684. Wright RS, Anderson JW \& Bridges SR (1990) Propionate inhibits hepatocyte lipid synthesis. Proc Soc Exp Biol Med 195, 26-29.

685. Demir G, Klein HO, Mandel-Molinas N, et al. (2007) $\beta$ Glucan induces proliferation and activation of monocytes in peripheral blood of patients with advanced breast cancer. Int Immunopharmacol 7, 113-116.

686. Vucenik I \& Shamsuddin AM (2003) Cancer inhibition by inositol hexaphosphate (IP6) and inositol: from laboratory to clinic. J Nutr 133, 3778S-3784S.

687. Muraoka S \& Miura T (2004) Inhibition of xanthine oxidase by phytic acid and its antioxidative action. Life Sci 74, 1691-1700.

688. Lee SH, Park HJ, Chun HK, et al. (2006) Dietary phytic acid lowers the blood glucose level in diabetic KK mice. Nutr Res 26, 474-479.

689. Lee S-H, Park H-J, Chun H-K, et al. (2007) Dietary phytic acid improves serum and hepatic lipid levels in aged ICR mice fed a high-cholesterol diet. Nutr Res 27, 505-510.

690. Onomi S, Okazaki Y \& Katayama T (2004) Effect of dietary level of phytic acid on hepatic and serum lipid status in rats fed a high-sucrose diet. Biosci Biotechnol Biochem 68, 1379-1381.

691. Lee SH, Park HJ, Cho SY, et al. (2005) Effects of dietary phytic acid on serum and hepatic lipid levels in diabetic KK mice. Nutr Res 25, 869-876.

692. Singh A, Prakash Singh S \& Bamezai R (1997) Modulatory influence of arecoline on the phytic acid-altered hepatic biotransformation system enzymes, sulfhydryl content and lipid peroxidation in a murine system. Cancer Lett 117, 1-6.

693. Grases F, Simonet BM, March JG, et al. (2000) Inositol hexakisphosphate in urine: the relationship between oral intake and urinary excretion. BJU Int 85, 138-142.

694. Grases F, Sanchis P, Perello J, et al. (2008) Phytate reduces age-related cardiovascular calcification. Front Biosci 13, $7115-7122$.

695. Shen XT, Xiao H, Ranallo R, et al. (2003) Modulation of ATP-dependent chromatin remodeling complexes by inositol polyphosphates. Science 299, 112-114.

696. Steger DJ, Haswell ES, Miller AL, et al. (2003) Regulation of chromatin remodeling by inositol polyphosphates. Science 299, 114-116.

697. Sajilata MG, Singhal RS \& Kulkarni PR (2006) Resistant starch: a review. Compr Rev Food Sci Food Saf 5, 1-17.

698. Malhotra SL (1968) Epidemiological study of cholelithiasis among railroad workers in India with special reference to causation. Gut 9, 290-295.

699. Beard JL \& Connor JR (2003) Iron status and neural functioning. Annu Rev Nutr 23, 41-58.

700. Institute of Medicine (2001) Dietary Reference Intake for Vitamin A, Vitamin K, Arsenic, Baron, Chromium, Copper, Iodine, Iron, Manganese, Molybdenum, Nickel, Silicon, Vanadium, and Zinc. Washington, DC: National Academy Press.

701. Uehara M, Chiba H, Mogi H, et al. (1997) Induction of increased phosphatidylcholine hydroperoxide by an iron-deficient diet in rats. J Nutr Biochem 8, 385-391.

702. Rosenzweig P \& Volpe S (1999) Iron, thermoregulation, and metabolic rate. Crit Rev Food Sci Nutr 39, 131-148.

703. Oexle H, Gnaiger E \& Weiss G (1999) Iron-dependent changes in cellular energy metabolism: influence on citric acid cycle and oxidative phosphorylation. Biochim Biophys Acta 1413, 99-107.

704. Ramdath DD \& Golden MHN (1989) Non-haematological aspects of iron nutrition. Nutr Res Rev 2, 29-49.
705. Lozoff B, Jimenez E, Hagen J, et al. (2000) Poorer behavioral and developmental outcome more than 10 years after treatment for iron deficiency in infancy. Pediatrics 105, E51.

706. Oski FA, Honig AS, Helu B, et al. (1983) Effect of iron therapy on behavior performance in nonanemic, irondeficient infants. Pediatrics 71, 877-880.

707. Prockop DJ (1971) Role of iron in synthesis of collagen in connective tissue. Fed Proc 30, 984-990.

708. Katsumata S, Katsumata-Tsuboi R, Uehara M, et al (2009) Severe iron deficiency decreases both bone formation and bone resorption in rats. J Nutr 139, $238-243$.

709. Willis WT, Dallman PR \& Brooks GA (1988) Physiological and biochemical correlates of increased work in trained iron-deficient rats. J Appl Physiol 65, 256-263.

710. Cook J \& Lynch S (1986) The liabilities of iron deficiency. Blood 68, 803-809.

711. Rosales FJ, Jang J-T, Pinero DJ, et al. (1999) Iron deficiency in young rats alters the distribution of vitamin A between plasma and liver and between hepatic retinol and retinyl esters. J Nutr 129, 1223-1228.

712. McClung JP \& Karl JP (2009) Iron deficiency and obesity: the contribution of inflammation and diminished iron absorption. Nutr Rev 67, 100-104.

713. Clancaglini P, Plzauro JM, Curti C, et al. (1990) Effect of membrane moiety and magnesium ions on the inhibition of matrix-induced alkaline phosphatase by zinc ions. Int $J$ Biochem 22, 747-751.

714. Bussiere FI, Gueux E, Rock E, et al. (2002) Increased phagocytosis and production of reactive oxygen species by neutrophils during magnesium deficiency in rats and inhibition by high magnesium concentration. Br J Nutr $\mathbf{8 7}$, 107-113.

715. Olatunji LA \& Soladoye AO (2007) Increased magnesium intake prevents hyperlipidemia and insulin resistance and reduces lipid peroxidation in fructose-fed rats. Pathophysiology 14, 11-15.

716. Kisters K, Spieker C, Tepel M, et al. (1993) New data about the effects of oral physiological magnesium supplementation on several cardiovascular risk factors (lipids and blood pressure). Magnes Res 6, 355-360.

717. Barbagallo M \& Dominguez LJ (2007) Magnesium metabolism in type 2 diabetes mellitus, metabolic syndrome and insulin resistance. Arch Biochem Biophys 458, 40-47.

718. Colditz G, Manson J, Stampfer M, et al. (1992) Diet and risk of clinical diabetes in women. Am J Clin Nutr $\mathbf{5 5}$, $1018-1023$.

719. Nadler JL, Balon TW \& Rude R (1997) Fiber intake and risk of developing non-insulin-dependent diabetes mellitus. JAMA 277, 1761-1762.

720. Paolisso G, Scheen A, D'Onofrio F, et al. (1990) Magnesium and glucose homeostasis. Diabetologia 33, $511-514$.

721. Schulze MB, Schulz M, Heidemann C, et al. (2007) Fiber and magnesium intake and incidence of type 2 diabetes: a prospective study and meta-analysis. Arch Intern Med 167, 956-965.

722. van Dam RM, Hu FB, Rosenberg L, et al. (2006) Dietary calcium and magnesium, major food sources, and risk of type 2 diabetes in U.S. black women. Diabetes Care 29, 2238-2243.

723. Paolisso G, Dimaro G, Cozzolino D, et al. (1992) Chronic magnesium administration enhances oxidative glucose metabolism in thiazide treated hypertensive patients. Am J Hypertens 5, 681-686. 
724. Nadler J, Buchanan T, Natarajan R, et al. (1993) Magnesium deficiency produces insulin resistance and increased thromboxane synthesis. Hypertension 21, 1024-1029.

725. Ascherio A, Rimm E, Giovannucci E, et al. (1992) A prospective study of nutritional factors and hypertension among US men. Circulation 86, 1475-1484.

726. Rubenowitz E, Axelsson G \& Rylander R (1996) Magnesium in drinking water and death from acute myocardial infarction. Am J Epidemiol 143, 456-462.

727. Cohen L (1988) Recent data on magnesium and osteoporosis. Magnes Res 1, 85-87.

728. Bernardini D, Nasulewicz A, Mazur A, et al. (2005) Magnesium and microvascular endothelial cells: a role in inflammation and angiogenesis. Front Biosci 10, 1177-1182.

729. Reungjui S, Prasongwatana V, Premgamone A, et al. (2002) Magnesium status of patients with renal stones and its effect on urinary citrate excretion. BJU Int $\mathbf{9 0}$, 635-639.

730. Bray TM \& Bettger WJ (1990) The physiological role of zinc as an antioxidant. Free Radic Biol Med 8, 281-291.

731. Zago MP \& Oteiza PI (2001) The antioxidant properties of zinc: interactions with iron and antioxidants. Free Radic Biol Med 31, 266-274.

732. Beattie JH \& Avenell A (1992) Trace element nutrition and bone metabolism. Nutr Res Rev 5, 167-188.

733. Ding W-Q, Yu H-J \& Lind SE (2008) Zinc-binding compounds induce cancer cell death via distinct modes of action. Cancer Lett 271, 251-259.

734. Guo W, Zhao Y-P, Jiang Y-G, et al. (2008) Restoring the metabolic disturbance of zinc: may not only contribute to the prevention of esophageal squamous cell cancer. Med Hypotheses 71, 957-959.

735. Hershfinkel M, Silverman WF \& Sekler I (2007) The zinc sensing receptor, a link between zinc and cell signaling. Mol Med 13, 331-336.

736. Bogden J, Oleske J, Munves E, et al. (1987) Zinc and immunocompetence in the elderly: baseline data on zinc nutriture and immunity in unsupplemented subjects. Am J Clin Nutr 46, 101-109.

737. Shen H, Oesterling E, Stromberg A, et al. (2008) Zinc deficiency induces vascular pro-inflammatory parameters

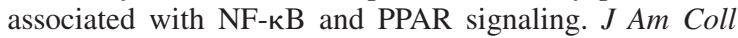
Nutr 27, 577-587.

738. Mocchegiani E, Giacconi R \& Malavolta M (2008) Zinc signalling and subcellular distribution: emerging targets in type 2 diabetes. Trends $\mathrm{Mol}$ Med 14, 419-428.

739. Ohinata K, Takemoto M, Kawanago M, et al. (2009) Orally administered zinc increases food intake via vagal stimulation in rats. J Nutr 139, 611-616.

740. Robinson BH (1998) The role of manganese superoxide dismutase in health and disease. J Inher Metab Dis 21 , 598-603.

741. Freeland-Graves JH \& Turnlund JR (1996) Deliberations and evaluations of the approaches, endpoints and paradigms for manganese and molybdenum dietary recommendations. J Nutr 126, 2435S-2440S.

742. Cho SJ, Park JW, Kang JS, et al. (2008) Nuclear factor-кB dependency of doxorubicin sensitivity in gastric cancer cells is determined by manganese superoxide dismutase expression. Cancer Sci 99, 1117-1124.

743. Kattan Z, Minig V, Dauça M, et al. (2007) Role of manganese superoxide dismutase on growth and invasive properties of human estrogen-independent breast cancer cells. Eur J Cancer Suppl 5, 76-77.
744. Johnson MA, Fischer JG \& Kays SE (1992) Is copper an antioxidant nutrient? Crit Rev Food Sci Nutr 32, 1-31.

745. Baker A, Harvey L, Majask-Newman G, et al. (1999) Effect of dietary copper intakes on biochemical markers of bone metabolism in healthy adult males. Eur J Clin Nutr 53, 408-412.

746. Lukaski HC, Klevay LM \& Milne DB (1988) Effects of dietary copper on human autonomic cardiovascular function. EurJ Appl Physiol 58, 74-80.

747. Milne D (1998) Copper intake and assessment of copper status. Am J Clin Nutr 67, 1041S-1045S.

748. Klevay LM (2006) Heart failure improvement from a supplement containing copper. Eur Heart J 27, 117-118.

749. Zhou Y, Jiang Y \& Kang YJ (2008) Copper reverses cardiomyocyte hypertrophy through vascular endothelial growth factor-mediated reduction in the cell size. $\mathrm{J} \mathrm{Mol}$ Cell Cardiol 45, 106-117.

750. Hammud HH, Nemer G, Sawma W, et al. (2008) Copperadenine complex, a compound, with multi-biochemical targets and potential anti-cancer effect. Chem Biol Interact 173, 84-96.

751. Klevay L (1975) Coronary heart disease: the zinc/copper hypothesis. Am J Clin Nutr 28, 764-774.

752. Klevay LM (1977) Hypo-cholesterolemia due to sodium phytate. Nutr Rep Int 15, 587-595.

753. Tapiero H, Townsend DM \& Tew KD (2003) The antioxidant role of selenium and seleno-compounds. Biomed Pharmacother 57, 134-144.

754. Levander OA (1992) Selenium and sulfur in antioxidant protective systems - relationships with vitamin $\mathrm{E}$ and malaria. Proc Soc Exp Biol Med 200, 255-259.

755. Burk RF (1990) Protection against free-radical injury by selenoenzymes. Pharmacol Ther 45, 383-385.

756. Jacobs MM (1977) Inhibitory effects of selenium on 1,2-dimethylhydrazine and methylazoxymethanol colon carcinogenesis. Correlative studies on selenium effects on the mutagenicity and sister chromatid exchange rates of selected carcinogens. Cancer 40, 2557-2564.

757. Jacobs MM, Forst CF \& Beams FA (1981) Biochemical and clinical effects of selenium on dimethylhydrazineinduced colon cancer in rats. Cancer Res 41, 4458-4465.

758. Jariwalla RJ, Gangapurkar B \& Nakamura D (2009) Differential sensitivity of various human tumour-derived cell types to apoptosis by organic derivatives of selenium. Br J Nutr 101, 182-189.

759. Gromadzinska J, Reszka E, Bruzelius K, et al. (2008) Selenium and cancer: biomarkers of selenium status and molecular action of selenium supplements. Eur J Nutr 47, 29-50.

760. Levander O \& Morris V (1984) Dietary selenium levels needed to maintain balance in North American adults consuming self-selected diets. Am J Clin Nutr 39, 809-815.

761. Arvilommi H, Poikonen K, Jokinen I, et al. (1983) Selenium and immune functions in humans. Infect Immun 41, 185-189.

762. Boyne R \& Arthur JR (1986) The response of seleniumdeficient mice to Candida albicans infection. J Nutr 116, 816-822.

763. Ciappellano S, Testolin G \& Porrini M (1989) Effects of durum wheat dietary selenium on glutathione peroxidase activity and Se content in long-term-fed rats. Ann Nutr Metab 33, 22-30.

764. Douillet C, Bost M, Accominotti M, et al. (1998) Effect of selenium and vitamin $\mathrm{E}$ supplementation on lipid abnormalities in plasma, aorta, and adipose tissue of Zucker rats. Biol Trace Elem Res 65, 221-236. 
765. Stapleton SR (2000) Selenium: an insulin mimetic. Cell Mol Life Sci 57, 1874-1879.

766. Uribarri J (2007) Phosphorus homeostasis in normal health and in chronic kidney disease patients with special emphasis on dietary phosphorus intake. Semin Dial 20, 295-301.

767. Loghman-Adham M (1997) Adaptation to changes in dietary phosphorus intake in health and in renal failure. J Lab Clin Med 129, 176-188.

768. Kesse E, Boutron-Ruault M-C, Norat T, et al. (2005) Dietary calcium, phosphorus, vitamin $\mathrm{D}$, dairy products and the risk of colorectal adenoma and cancer among French women of the E3N-EPIC prospective study. Int $J$ Cancer 117, 137-144.

769. Arnold WH \& Gaengler P (2007) Quantitative analysis of the calcium and phosphorus content of developing and permanent human teeth. Ann Anat 189, 183-190.

770. Bostick RM, Potter JD, Fosdick L, et al. (1993) Calcium and colorectal epithelial cell proliferation: a preliminary randomized, double-blinded, placebo-controlled clinical trial. J Natl Cancer Inst 85, 132-141.

771. Ishihara J, Inoue M, Iwasaki M, et al. (2008) Dietary calcium, vitamin $\mathrm{D}$, and the risk of colorectal cancer. Am J Clin Nutr 88, 1576-1583.

772. Mariot P, Vanoverberghe K, Lalevee N, et al. (2002) Overexpression of an $\alpha{ }^{1} \mathrm{H}$ (Cav3.2) T-type calcium channel during neuroendocrine differentiation of human prostate cancer cells. J Biol Chem 277, 10824-10833.

773. Taylor JT, Zeng XB, Pottle JE, et al. (2008) Calcium signaling and T-type calcium channels in cancer cell cycling. World J Gastroenterol 14, 4984-4991.

774. Ciapa B, Pesando D, Wilding M, et al. (1994) Cell-cycle calcium transients driven by cyclic changes in inositol trisphosphate levels. Nature 368, 875-878.

775. Bucher HC, Cook RJ, Guyatt GH, et al. (1996) Effects of dietary calcium supplementation on blood pressure. A meta-analysis of randomized controlled trials. JAMA 275, 1016-1022.

776. Gillman MW, Hood MY, Moore LL, et al. (1995) Effect of calcium supplementation on blood pressure in children. J Pediatr 127, 186-192.

777. Umesawa M, Iso H, Ishihara J, et al. (2008) Dietary calcium intake and risks of stroke, its subtypes, and coronary heart disease in Japanese: The JPHC Study Cohort. Stroke 39, 2449-2456.

778. Astrup A (2008) The role of calcium in energy balance and obesity: the search for mechanisms. Am J Clin Nutr 88, 873-874.

779. Major GC, Chaput JP, Ledoux M, et al. (2008) Recent developments in calcium-related obesity research. Obes $\operatorname{Rev}$ 9, 428-445.

780. Sharp RL (2006) Role of sodium in fluid homeostasis with exercise. J Am Coll Nutr 25, 231S-239S.

781. Hollenberg NK (2006) The influence of dietary sodium on blood pressure. J Am Coll Nutr 25, 240S-246S.

782. Alderman MH (2006) Evidence relating dietary sodium to cardiovascular disease. J Am Coll Nutr 25, 256S-261S.

783. Heaney RP (2006) Role of dietary sodium in osteoporosis. $J$ Am Coll Nutr 25, 271S-276S.

784. Demigne C, Sabboh H, Remesy C, et al. (2004) Protective effects of high dietary potassium: nutritional and metabolic aspects. J Nutr 134, 2903-2906.

785. He FJ \& MacGregor GA (2008) Beneficial effects of potassium on human health. Physiologia Plantarum 133, $725-735$.

786. Sjogren A, Floren CH \& Nilsson A (1988) Oral administration of magnesium hydroxide to subjects with insulin-dependent diabetes mellitus - effects on magnesium and potassium levels and on insulin requirements. Magnesium 7, 117-122.

787. Appel LJ, Moore TJ, Obarzanek E, et al. (1997) A clinical trial of the effects of dietary patterns on blood pressure. $N$ Engl J Med 336, 1117-1124.

788. Chang H-Y, Hu Y-W, Yue C-SJ, et al. (2006) Effect of potassium-enriched salt on cardiovascular mortality and medical expenses of elderly men. Am J Clin Nutr 83, 1289-1296.

789. Ishimitsu T, Tobian L, Sugimoto KI, et al. (1995) High potassium diets reduce macrophage adherence to the vascular wall in stroke-prone spontaneously hypertensive rats. J Vasc Res 32, 406-412.

790. Young DB \& Ma G (1999) Vascular protective effects of potassium. Semin Nephrol 19, 477-486.

791. Nolan J, Batin PD, Andrews R, et al. (1998) Prospective study of heart rate variability and mortality in chronic heart failure: results of the United Kingdom Heart Failure Evaluation and Assessment of Risk Trial (UK-Heart). Circulation 98, 1510-1516.

792. Tobian L, Macneill D, Johnson MA, et al. (1984) Potassium protection against lesions of the renal tubules, arteries, and glomeruli and nephron loss in salt-loaded hypertensive Dahl S-rats. Hypertension 6, I170-I176.

793. Curhan GC, Willett WC, Rimm EB, et al. (1993) A prospective study of dietary calcium and other nutrients and the risk of symptomatic kidney stones. $N$ Engl $\mathrm{J} \mathrm{Med}$ 328, 833-838.

794. Marangella M, Di Stefano M, Casalis S, et al. (2004) Effects of potassium citrate supplementation on bone metabolism. Calcif Tissue Int 74, 330-335.

795. Lemann J Jr, Pleuss JA, Gray RW, et al. (1991) Potassium administration increases and potassium deprivation reduces urinary calcium excretion in healthy adults. Kidney Int 39, 973-983.

796. Institute of Medicine (1998) Dietary Reference Intakes for Thiamin, Riboflavin, Niacin, Vitamin $B_{6}$, Folate, Vitamin $B_{12}$, Pantothenic Acid, Biotin, and Choline. Washington, DC: National Academy Press.

797. Singleton CK \& Martin PR (2001) Molecular mechanisms of thiamine utilization. Curr Mol Med 1, 197-207.

798. Lukienko P, Mel'nichenko N, Zverinskii I, et al. (2000) Antioxidant properties of thiamine. Bull Exp Biol Med 130, 874-876.

799. Andreasen MF, Christensen LP, Meyer AS, et al. (2000) Content of phenolic acids and ferulic acid dehydrodimers in 17 rye (Secale cereale L.) varieties. J Agric Food Chem 48, 2837-2842.

800. Ambrose ML, Bowden SC \& Whelan G (2001) Thiamin treatment and working memory function of alcoholdependent people: preliminary findings. Alcohol Clin Exp Res 25, 112-116.

801. Fairweather-Tait SJ, Powers HJ, Minski MJ, et al. (1992) Riboflavin deficiency and iron absorption in adult Gambian men. Ann Nutr Metab 36, 34-40.

802. Sirivech S, Driskell J \& Frieden E (1977) NADH-FMN oxidoreductase activity and iron content of organs from riboflavin- and iron-deficient rats. J Nutr 107, 739-745.

803. Yates CA, Evans GS \& Powers HJ (2001) Riboflavin deficiency: early effects on post-weaning development of the duodenum in rats. Br J Nutr 86, 593-599.

804. Sterner RT \& Price WR (1973) Restricted riboflavin: within-subject behavioral effects in humans. Am J Clin Nutr 26, 150-160.

805. Miyamoto Y \& Sancar A (1998) Vitamin $B_{2}$-based bluelight photoreceptors in the retinohypothalamic tract as the 
photoactive pigments for setting the circadian clock in mammals. Proc Natl Acad Sci U S A 95, 6097-6102.

806. Mack CP, Hultquist DE \& Shlafer M (1995) Myocardial flavin reductase and riboflavin: a potential role in decreasing reoxygenation injury. Biochem Biophys Res Commun 212, 35-40.

807. Powers HJ (2003) Riboflavin (vitamin B-2) and health. Am J Clin Nutr 77, 1352-1360.

808. Siassi F \& Ghadirian P (2005) Riboflavin deficiency and esophageal cancer: a case control-household study in the Caspian Littoral of Iran. Cancer Detect Prev 29, 464-469.

809. Webster RP, Gawde MD \& Bhattacharya RK (1996) Modulation of carcinogen-induced DNA damage and repair enzyme activity by dietary riboflavin. Cancer Lett 98, 129-135.

810. Figge HL, Figge J, Souney PF, et al. (1988) Nicotinic acid - a review of its clinical use in the treatment of lipid disorders. Pharmacotherapy 8, 287-294.

811. Hodis HN (1995) Reversibility of atherosclerosis evolving perspectives from two arterial imaging clinical trials: The Cholesterol Lowering Atherosclerosis Regression study and the Monitored Atherosclerosis Regression study. J Cardiovasc Pharmacol 25, S25-S31.

812. Jacobson EL, Dame AJ, Pyrek JS, et al. (1995) Evaluating the role of niacin in human carcinogenesis. Biochimie 77, 394-398.

813. Pontes Monteiro J, Ferreira da Cunha D, Correia Filho D, et al. (2004) Niacin metabolite excretion in alcoholic pellagra and AIDS patients with and without diarrhea. Nutrition 20, 778-782.

814. Jonas W, Rapoza C \& Blair W (1996) The effect of niacinamide on osteoarthritis: a pilot study. Inflamm Res 45, 330-334.

815. Carlson LA (1963) Studies on effect of nicotinic acid on catecholamine stimulated lipolysis in adipose tissue in vitro. Acta Med Scand 173, 719-722.

816. Davies JI \& Souness JE (1981) The mechanisms of hormone and drug actions on fatty acid release from adipose tissue. Rev Pure Appl Pharmacol Sci 2, 1-112.

817. Marcus C, Sonnenfeld T, Karpe B, et al. (1989) Inhibition of lipolysis by agents acting via adenylate cyclase in fat cells from infants and adults. Pediatr Res 26, 255-259.

818. Anguita M, Gasa J, Martin-Orue SM, et al. (2006) Study of the effect of technological processes on starch hydrolysis, non-starch polysaccharides solubilization and physicochemical properties of different ingredients using a twostep in vitro system. Anim Feed Sci Tech 129, 99-115.

819. Ubbink JB, Becker PJ \& Vermaak WJH (1996) Will an increased dietary folate intake reduce the incidence of cardiovascular disease? Nutr Rev 54, 213-216.

820. Brattström L, Israelsson B, Norrving B, et al. (1990) Impaired homocysteine metabolism in early-onset cerebral and peripheral occlusive arterial disease. Effects of pyridoxine and folic acid treatment. Atherosclerosis 81, $51-60$.

821. McMahon RJ (2002) Biotin in metabolism and molecular biology. Annu Rev Nutr 22, 221-239.

822. Said HM (2009) Cell and molecular aspects of human intestinal biotin absorption. J Nutr 139, 158-162.

823. Sweetman L \& Nyhan WL (1986) Inheritable biotintreatable disorders and associated phenomena. Annu Rev Nutr 6, 317-343.

824. Rodriguez-Melendez R \& Zempleni J (2003) Regulation of gene expression by biotin (review). J Nutr Biochem 14, 680-690.

825. Manthey KC, Griffin JB \& Zempleni J (2002) Biotin supply affects expression of biotin transporters, biotinylation of carboxylases and metabolism of interleukin-2 in Jurkat cells. J Nutr 132, 887-892.

826. Baez-Saldana A, Diaz G, Espinoza B, et al. (1998) Biotin deficiency induces changes in subpopulations of spleen lymphocytes in mice. Am J Clin Nutr 67, 431-437.

827. Rabin BS (1983) Inhibition of experimentally induced autoimmunity in rats by biotin deficiency. $J$ Nutr 113, 2316-2322.

828. Kamen B (1997) Folate and antifolate pharmacology. Semin Oncol 24, 30-39.

829. Moat SJ, Hill MH, McDowell IFW, et al. (2003) Reduction in plasma total homocysteine through increasing folate intake in healthy individuals is not associated with changes in measures of antioxidant activity or oxidant damage. Eur J Clin Nutr 57, 483-489.

830. Ward M, McNulty H, McPartlin J, et al. (1997) Plasma homocysteine, a risk factor for cardiovascular disease, is lowered by physiological doses of folic acid. QJM 90, 519-524.

831. Shaw GM, Schaffer D, Velie EM, et al. (1995) Periconceptional vitamin use, dietary folate, and the occurrence of neural tube defects. Epidemiology 6, 219-226.

832. Giovannucci E (2002) Epidemiologic studies of folate and colorectal neoplasia: a review. J Nutr 132, 2350S-2355S.

833. Jennings E (1995) Folic acid as a cancer-preventing agent. Med Hypotheses 45, 297-303.

834. Macgregor JT, Schlegel R, Wehr CM, et al. (1990) Cytogenetic damage induced by folate deficiency in mice is enhanced by caffeine. Proc Natl Acad Sci U S A 87, 9962-9965.

835. Akilzhanova A, Takamura N, Aoyagi K, et al. (2006) Folic acid deficiency: main etiological factor of megaloblastic anemia in Kazakhstan? Am J Hematol 81, 471.

836. Ebisch IMW, Thomas CMG, Peters WHM, et al. (2007) The importance of folate, zinc and antioxidants in the pathogenesis and prevention of subfertility. Hum Reprod Update 13, 163-174.

837. Zeisel SH (2009) Importance of methyl donors during reproduction. Am J Clin Nutr 89, 673S-677S.

838. Gey KF (1998) Vitamins E plus C and interacting conutrients required for optimal health: a critical and constructive review of epidemiology and supplementation data regarding cardiovascular disease and cancer. Biofactors 7, 113-174.

839. Leger CL (2000) Prevention of cardiovascular risk by vitamin E. Ann Biol Clin 58, 527-540.

840. Bowry VW \& Ingold KU (1999) The unexpected role of vitamin $\mathrm{E}$ ( $\alpha$-tocopherol) in the peroxidation of human low-density lipoprotein. Acc Chem Res 32, 27-34.

841. Duthie SJ, Ma A, Ross MA, et al. (1996) Antioxidant supplementation decreases oxidative DNA damage in human lymphocytes. Cancer Res 56, 1291-1295.

842. Poulin JE, Cover C, Gustafson MR, et al. (1996) Vitamin E prevents oxidative modification of brain and lymphocyte band 3 proteins during aging. Proc Natl Acad Sci U S A 93, 5600-5603.

843. Yu WP, Simmons-Menchaca M, Gapor A, et al. (1999) Induction of apoptosis in human breast cancer cells by tocopherols and tocotrienols. Nutr Cancer 33, 26-32.

844. Azzi A \& Stocker A (2000) Vitamin E: non-antioxidant roles. Prog Lipid Res 39, 231-255.

845. Tasinato A, Boscoboinik D, Bartoli GM, et al. (1995) $D-\alpha$-Tocopherol inhibition of vascular smooth muscle cell proliferation occurs at physiological concentrations, correlates with protein kinase $\mathrm{C}$ inhibition, and is 
independent of its antioxidant properties. Proc Natl Acad Sci U S A 92, 12190-12194.

846. Devaraj S \& Jialal I (1999) $\alpha$-Tocopherol decreases interleukin- $1 \beta$ release from activated human monocytes by inhibition of 5-lipoxygenase. Arterioscler Thromb Vasc Biol 19, 1125-1133.

847. Ricciarelli R, Zingg J-M \& Azzi A (2000) Vitamin E reduces the uptake of oxidized LDL by inhibiting CD36 scavenger receptor expression in cultured aortic smooth muscle cells. Circulation 102, 82-87.

848. Teupser D, Thiery J \& Seidel D (1999) $\alpha$-Tocopherol down-regulates scavenger receptor activity in macrophages. Atherosclerosis 144, 109-115.

849. Christen S, Woodall AA, Shigenaga MK, et al. (1997) $\gamma$-Tocopherol traps mutagenic electrophiles such as $\mathrm{NO}_{\mathrm{x}}$ and complements $\alpha$-tocopherol: physiological implications. Proc Natl Acad Sci U S A 94, 3217-3222.

850. Wolf $\mathrm{G}$ (1997) $\gamma$-Tocopherol: an efficient protector of lipids against nitric oxide-initiated peroxidative damage. Nutr Rev 55, 376-378.

851. Chatelain E, Boscoboinik DO, Bartoli GM, et al. (1993) Inhibition of smooth muscle cell proliferation and protein kinase $\mathrm{C}$ activity by tocopherols and tocotrienols. Biochim Biophys Acta 1176, 83-89.

852. Stolzenberg-Solomon RZ, Sheffler-Collins S, Weinstein S, et al. (2009) Vitamin E intake, $\alpha$-tocopherol status, and pancreatic cancer in a cohort of male smokers. Am J Clin Nutr 89, 584-591.

853. Laight DW, Desai KM, Gopaul NK, et al. (1999) F2isoprostane evidence of oxidant stress in the insulin resistant, obese Zucker rat: effects of vitamin E. Eur $J$ Pharmacol 377, 89-92.

854. Ima-Nirwana S \& Suhaniza S (2004) Effects of tocopherols and tocotrienols on body composition and bone calcium content in adrenalectomized rats replaced with dexamethasone. J Med Food 7, 45-51.

855. Norazlina M, Ima-Nirwana S, Gapor MTA, et al. (2002) Tocotrienols are needed for normal bone calcification in growing female rats. Asia Pac J Clin Nutr 11, 194-199.

856. Suttie JW (1992) Vitamin K and human nutrition. J Am Diet Assoc 92, 585-590.

857. Suttie J (1993) Synthesis of vitamin K-dependent proteins. FASEB J 7, 445-452.

858. Hodges SJ, Akesson K, Vergnaud P, et al. (1993) Circulating levels of vitamin $\mathrm{K}_{1}$ and vitamin $\mathrm{K}_{2}$ decreased in elderly women with hip fracture. J Bone Miner Res $\mathbf{8}$, $1241-1245$.

859. Luo G, Ducy P, McKee MD, et al. (1997) Spontaneous calcification of arteries and cartilage in mice lacking matrix GLA protein. Nature 386, 78-81.

860. Mayne S (1996) $\beta$-Carotene, carotenoids, and disease prevention in humans. FASEB $J$ 10, 690-701.

861. Baron JA, Cole BF, Mott L, et al. (2003) Neoplastic and antineoplastic effects of $\beta$-carotene on colorectal adenoma recurrence: results of a randomized trial. J Natl Cancer Inst 95, 717-722.

862. Holick CN, Michaud DS, Stolzenberg-Solomon R, et al. (2002) Dietary carotenoids, serum $\beta$-carotene, and retinol and risk of lung cancer in the Alpha-Tocopherol, BetaCarotene Cohort Study. Am J Epidemiol 156, 536-547.

863. Michaud DS, Feskanich D, Rimm EB, et al. (2000) Intake of specific carotenoids and risk of lung cancer in 2 prospective US cohorts. Am J Clin Nutr 72, 990-997.

864. Touvier M, Kesse E, Clavel-Chapelon F, et al. (2005) Dual association of $\beta$-carotene with risk of tobacco-related cancers in a cohort of French women. J Natl Cancer Inst 97, $1338-1344$.
865. Rettura G, Duttagupta C, Listowsky P, et al. (1983) Dimethylbenz(a)anthracene (DMBA)-induced tumors: prevention by supplemental $\beta$-carotene (BC). Fed Proc 42, 786.

866. Cui Y, Lu Z, Bai L, et al. (2007) $\beta$-Carotene induces apoptosis and up-regulates peroxisome proliferatoractivated receptor $\gamma$ expression and reactive oxygen species production in MCF-7 cancer cells. Eur J Cancer 43, 2590-2601.

867. Prabhala RH, Braune LM, Garewal HS, et al. (1993) Influence of $\beta$-carotene on immune functions. Ann $N Y$ Acad Sci 691, 262-263.

868. Packer JE, Mahood JS, Mora-Arellano VO, et al. (1981) Free radicals and singlet oxygen scavengers: reaction of a peroxy-radical with $\beta$-carotene, diphenyl furan and 1,4diazobicyclo(2,2,2)-octane. Biochem Biophys Res Commun 98, 901-906.

869. Osganian SK, Stampfer MJ, Rimm E, et al. (2003) Dietary carotenoids and risk of coronary artery disease in women. Am J Clin Nutr 77, 1390-1399.

870. Granado F, Olmedilla B \& Blanco I (2003) Nutritional and clinical relevance of lutein in human health. Br J Nutr $\mathbf{9 0}$, 487-502.

871. Stringheta PC, Nachtigall AM, Oliveira TT, et al. (2006) Lutein: antioxidant properties and health benefits. Alimentos e Nutricao 17, 229-237.

872. Richer S, Stiles W, Statkute L, et al. (2004) Doublemasked, placebo-controlled, randomized trial of lutein and antioxidant supplementation in the intervention of atrophic age-related macular degeneration: the Veterans LAST study (Lutein Antioxidant Supplementation Trial). Optometry 75, 216-229.

873. Seddon JM, Ajani UA, Sperduto RD, et al. (1994) Dietary carotenoids, vitamins $\mathrm{A}, \mathrm{C}$, and $\mathrm{E}$, and advanced agerelated macular degeneration. Eye Disease Case-Control Study Group. JAMA 272, 1413-1420.

874. Olmedilla B, Granado F, Blanco I, et al. (2003) Lutein, but not $\alpha$-tocopherol, supplementation improves visual function in patients with age-related cataracts: a 2-y doubleblind, placebo-controlled pilot study. Nutrition 19, 21-24.

875. Curran-Celentano J, Hammond BR Jr, Ciulla TA, et al. (2001) Relation between dietary intake, serum concentrations, and retinal concentrations of lutein and zeaxanthin in adults in a Midwest population. Am J Clin Nutr 74, 796-802.

876. Anonymous (2005) Lutein and zeaxanthin. Monograph. Altern Med Rev 10, 128-135.

877. Schäffer MW, Roy SS, Mukherjee S, et al. (2008) Identification of lutein, a dietary antioxidant carotenoid in guinea pig tissues. Biochem Biophys Res Commun 374, $378-381$.

878. Stahl W \& Sies H (2002) Carotenoids and protection against solar UV radiation. Skin Pharmacol Appl Skin Physiol 15, 291-296.

879. Slattery ML, Benson J, Curtin K, et al. (2000) Carotenoids and colon cancer. Am J Clin Nutr 71, 575-582.

880. Dwyer JH, Navab M, Dwyer KM, et al. (2001) Oxygenated carotenoid lutein and progression of early atherosclerosis: The Los Angeles Atherosclerosis Study. Circulation 103, 2922-2927.

881. Yeum K-J, Shang F, Schalch W, et al. (1999) Fat-soluble nutrient concentrations in different layers of human cataractous lens. Curr Eye Res 19, 502-505.

882. Uchiyama S, Sumida T \& Yamaguchi M (2004) Oral administration of $\beta$-cryptoxanthin induces anabolic effects on bone components in the femoral tissues of rats in vivo. Biol Pharm Bull 27, 232-235. 
883. Yamaguchi M \& Uchiyama S (2004) $\beta$-Cryptoxanthin stimulates bone formation and inhibits bone resorption in tissue culture in vitro. Mol Cell Biochem 258, 137-144.

884. Kohno H, Taima M, Sumida T, et al. (2001) Inhibitory effect of mandarin juice rich in $\beta$-cryptoxanthin and hesperidin on 4-(methylnitrosamino)-1-(3-pyridyl)-1butanone-induced pulmonary tumorigenesis in mice. Cancer Lett 174, 141-150.

885. Lian F, Kang-Quan Hu K-Q, Russell RM, et al. (2006) $\beta$-Cryptoxanthin suppresses the growth of immortalized human bronchial epithelial cells and non-small-cell lung cancer cells and up-regulates retinoic acid receptor $\beta$ expression. Int J Cancer 119, 2084-2089.

886. Yuan J-M, Ross RK, Chu X-D, et al. (2001) Prediagnostic levels of serum $\beta$-cryptoxanthin and retinol predict smoking-related lung cancer risk in Shanghai, China. Cancer Epidemiol Biomarkers Prev 10, 767-773.

887. Tanaka T, Kohno H, Murakami M, et al. (2000) Suppression of azoxymethane-induced colon carcinogenesis in male F344 rats by mandarin juices rich in $\beta$-cryptoxanthin and hesperidin. Int J Cancer 88, 146-150.

888. Nogushi S, Sumida T, Ogawa H, et al. (2003) Effects of oxygenated carotenoid $\beta$-cryptoxanthin on morphological differentiation and apoptosis in Neuro2a neuroblastoma cells. Biosci Biotechnol Biochem 67, 2467-2469.

889. Castelao JE \& Olmedilla B (2002) Vitamins A, C and E, folate and most carotenoids do not influence bladder cancer risk. Evid Based Oncol 3, 58-59.

890. Rice-Evans CA, Miller NJ \& Paganga G (1997) Antioxidant properties of phenolic compounds. Trends Plant Sci 2, 152-159.

891. Adisakwattana S, Moonsan P \& Yibchok-anun S (2008) Insulin-releasing properties of a series of cinnamic acid derivatives in vitro and in vivo. J Agric Food Chem 56, $7838-7844$.

892. Tanaka T, Kojima T, Kawamori T, et al. (1993) Inhibition of 4-nitroquinoline-1-oxide-induced rat tongue carcinogenesis by the naturally occurring plant phenolics caffeic, ellagic, chlorogenic and ferulic acids. Carcinogenesis 14, $1321-1325$.

893. Ferguson LR, Zhu ST \& Harris PJ (2005) Antioxidant and antigenotoxic effects of plant cell wall hydroxycinnamic acids in cultured HT-29 cells. Mol Nutr Food Res 49, 585-593.

894. Hsu C-L, Wu C-H, Huang S-L, et al. (2009) Phenolic compounds rutin and $o$-coumaric acid ameliorate obesity induced by high-fat diet in rats. J Agric Food Chem 57, $425-431$

895. Sri Balasubashini M, Rukkumani R, Viswanathan P, et al. (2004) Ferulic acid alleviates lipid peroxidation in diabetic rats. Phytother Res 18, 310-314.

896. Kamal-Eldin A, Frank J, Razdan A, et al. (2000) Effects of dietary phenolic compounds on tocopherol, cholesterol, and fatty acids in rats. Lipids $\mathbf{3 5}, 427-435$.

897. Sri Balasubashini M, Rukkumani R \& Menon VP (2003) Protective effects of ferulic acid on hyperlipidemic diabetic rats. Acta Diabetol 40, 118-122.

898. Suzuki A, Kagawa D, Fujii A, et al. (2002) Short- and long-term effects of ferulic acid on blood pressure in spontaneously hypertensive rats. Am J Hypertens 15, $351-357$.

899. Jung EH, Kim SR, Hwang IK, et al. (2007) Hypoglycemic effects of a phenolic acid fraction of rice bran and ferulic acid in C57BL/KsJ-db/db mice. J Agric Food Chem 55, 9800-9804.
900. Hollman PCH \& Katan MB (1997) Absorption, metabolism and health effects of dietary flavonoids in man. Biomed Pharmacother 51, 305-310.

901. Van Wauwe J \& Goossens J (1983) Effects of antioxidants on cyclooxygenase and lipoxygenase activities in intact human platelets: comparison with indomethacin and ETYA. Prostaglandins 26, 725-730.

902. Cushnie TPT \& Lamb AJ (2005) Antimicrobial activity of flavonoids. Int J Antimicrob Agents 26, 343-356.

903. Zhang G, Qin L, Hung WY, et al. (2006) Flavonoids derived from herbal Epimedium Brevicornum Maxim prevent OVX-induced osteoporosis in rats independent of its enhancement in intestinal calcium absorption. Bone 38, 818-825.

904. Chiang AN, Wu HL, Yeh HI, et al. (2006) Antioxidant effects of black rice extract through the induction of superoxide dismutase and catalase activities. Lipids 41, 797-803.

905. Ling WH, Wang LL \& Ma J (2002) Supplementation of the black rice outer layer fraction to rabbits decreases atherosclerotic plaque formation and increases antioxidant status. J Nutr 132, 20-26.

906. Nam SH, Choi SP, Kang MY, et al. (2006) Antioxidative activities of bran extracts from twenty one pigmented rice cultivars. Food Chem 94, 613-620.

907. Tsuda T, Horio F \& Osawa $T$ (2002) Cyanidin 3-O- $\beta$-D-glucoside suppresses nitric oxide production during a zymosan treatment in rats. J Nutr Sci Vitaminol 48, 305-310.

908. Xia XD, Ling WH, Ma J, et al. (2006) An anthocyanin-rich extract from black rice enhances atherosclerotic plaque stabilization in apolipoprotein E-deficient mice. J Nutr 136, 2220-2225.

909. Hyun JW \& Chung HS (2004) Cyanidin and malvidin from Oryza sativa cv. heugjinjubyeo mediate cytotoxicity against human monocytic leukemia cells by arrest of $\mathrm{G}_{2} / \mathrm{M}$ phase and induction of apoptosis. J Agric Food Chem 52, 2213-2217.

910. Zhao C, Giusti MM, Malik M, et al. (2004) Effects of commercial anthocyanin-rich extracts on colonic cancer and nontumorigenic colonic cell growth. J Agric Food Chem 52, 6122-6128.

911. Tsuda T, Horio F, Uchida K, et al. (2003) Dietary cyanidin 3-O- $\beta$-D-glucoside-rich purple corn color prevents obesity and ameliorates hyperglycemia in mice. J Nutr 133, 2125-2130.

912. Kaludjerovic J \& Ward WE (2009) Neonatal exposure to daidzein, genistein, or the combination modulates bone development in female CD-1 mice. J Nutr 139, 467-473.

913. Jenab M \& Thompson LU (1996) The influence of flaxseed and lignans on colon carcinogenesis and $\beta$-glucuronidase activity. Carcinogenesis 17, 1343-1348.

914. Oikarinen SI, Pajari A-M \& Mutanen M (2000) Chemopreventive activity of crude hydroxsymatairesinol (HMR) extract in ApcMin mice. Cancer Lett 161, 253-258.

915. Prasad K (2005) Hypocholesterolemic and antiatherosclerotic effect of flax lignan complex isolated from flaxseed. Atherosclerosis 179, 269-275.

916. Kamal-Eldin A, Pouru A, Eliasson C, et al. (2001) Alkylresorcinols as antioxidants: hydrogen donation and peroxyl radical-scavenging effects. J Sci Food Agric 81, 353-356.

917. Kozubek A \& Nienartowicz B (1995) Cereal grain resorcinolic lipids inhibit $\mathrm{H}_{2} \mathrm{O}_{2}$-induced peroxidation of biological membranes. Acta Biochim Pol 42, 309-315. 
918. Gasiorowski K, Szyba K, Brokos B, et al. (1996) Antimutagenic activity of alkylresorcinols from cereal grains. Cancer Lett 106, 109-115.

919. Olthof MR, van Vliet T, Boelsma E, et al. (2003) Low dose betaine supplementation leads to immediate and long term lowering of plasma homocysteine in healthy men and women. J Nutr 133, 4135-4138.

920. Handler JS \& Kwon HM (2001) Cell and molecular biology of organic osmolyte accumulation in hypertonic renal cells. Nephron 87, 106-110.

921. Konstantinova SV, Tell GS, Vollset SE, et al. (2008) Divergent associations of plasma choline and betaine with components of metabolic syndrome in middle age and elderly men and women. $J$ Nutr 138, 914-920.

922. Detopoulou P, Panagiotakos DB, Antonopoulou S, et al. (2008) Dietary choline and betaine intakes in relation to concentrations of inflammatory markers in healthy adults: the ATTICA study. Am J Clin Nutr 87, 424-430.

923. Lv S, Fan R, Du Y, et al. (2009) Betaine supplementation attenuates atherosclerotic lesion in apolipoprotein E-deficient mice. Eur J Nutr 48, 205-212.

924. Delgado-Reyes CV \& Garrow TA (2005) High sodium chloride intake decreases betaine-homocysteine $S$-methyltransferase expression in guinea pig liver and kidney. $A m \mathrm{~J}$ Physiol Regul Integr Comp Physiol 288, R182-R187.

925. Kwon DY, Jung YS, Kim SJ, et al. (2009) Impaired sulfur-amino acid metabolism and oxidative stress in nonalcoholic fatty liver are alleviated by betaine supplementation in rats. $J$ Nutr 139, 63-68.

926. Albright CD, Tsai AY, Friedrich CB, et al. (1999) Choline availability alters embryonic development of the hippocampus and septum in the rat. Dev Brain Res 113, 13-20.

927. Meck WH \& Williams CL (2003) Metabolic imprinting of choline by its availability during gestation: implications for memory and attentional processing across the lifespan. Neurosci Biobehav Rev 27, 385-399.

928. Sanders LM \& Zeisel SH (2007) Choline. Nutr Today 42 , $181-186$.

929. Cho E, Zeisel SH, Jacques P, et al. (2006) Dietary choline and betaine assessed by food-frequency questionnaire in relation to plasma total homocysteine concentration in the Framingham Offspring Study. Am J Clin Nutr 83, 905-911.

930. Sachan DS, Hongu N \& Johnsen M (2005) Decreasing oxidative stress with choline and carnitine in women. $\mathrm{JAm}$ Coll Nutr 24, 172-176.

931. Dodson W \& Sachan D (1996) Choline supplementation reduces urinary carnitine excretion in humans. Am J Clin Nutr 63, 904-910.

932. Daily JW, Hongu N, Mynatt RL, et al. (1998) Choline supplementation increases tissue concentrations of carnitine and lowers body fat in guinea pigs. J Nutr Biochem $\mathbf{9}$, 464-470.

933. Sachan DS \& Hongu N (2000) Increases in $\mathrm{VO}_{2}$ max and metabolic markers of fat oxidation by caffeine, carnitine, and choline supplementation in rats. J Nutr Biochem 11, 521-526.

934. Exton JH (1994) Phosphatidylcholine breakdown and signal transduction. Biochim Biophys Acta 1212, 26-42.

935. Hannun Y (1994) The sphingomyelin cycle and the second messenger function of ceramide. J Biol Chem 269 , $3125-3128$

936. Cohen EL \& Wurtman RJ (1975) Brain acetylcholine: increase after systematic choline administration. Life Sci 16, 1095-1102.
937. Frenkel RA, Muguruma K \& Johnston JM (1996) The biochemical role of platelet-activating factor in reproduction. Prog Lipid Res 35, 155-168.

938. Haubrich DR, Wedeking PW \& Wang PFL (1974) Increase in tissue concentration of acetylcholine in guinea pigs in vivo induced by administration of choline. Life Sci 14, 921-927.

939. Zeisel SH (2006) Choline: critical role during fetal development and dietary requirements in adults. Annu Rev Nutr 26, 229-250.

940. Henning SM \& Swendseid ME (1996) The role of folate, choline, and methionine in carcinogenesis induced by methyl-deficient diets. Adv Exp Med Biol 399, 143-155.

941. Niculescu MD, Craciunescu CN \& Zeisel SH (2006) Dietary choline deficiency alters global and gene-specific DNA methylation in the developing hippocampus of mouse fetal brains. FASEB $J$ 20, 43-49.

942. Brufau G, Canela MA \& Rafecas M (2008) Phytosterols: physiologic and metabolic aspects related to cholesterollowering properties. Nutr Res 28, 217-225.

943. de Jong A, Plat J \& Mensink RP (2003) Metabolic effects of plant sterols and stanols (review). J Nutr Biochem 14, 362-369.

944. Batta AK, Xu GR, Bollineni JS, et al. (2005) Effect of high plant sterol-enriched diet and cholesterol absorption inhibitor, $\mathrm{SCH}$ 58235, on plant sterol absorption and plasma concentrations in hypercholesterolemic wild-type Kyoto rats. Metabolism 54, 38-48.

945. Jones PJH, MacDougall DE, Ntanios F, et al. (1997) Dietary phytosterols as cholesterol-lowering agents in humans. Can J Physiol Pharmacol 75, 217-227.

946. Lee Y-M, Haastert B, Scherbaum W, et al. (2003) A phytosterol-enriched spread improves the lipid profile of subjects with type 2 diabetes mellitus. Eur J Nutr 42, $111-117$

947. Yankah VV \& Jones PJH (2001) Phytosterols and health implications - efficacy and nutritional aspects. Inform $\mathbf{1 2}$ 899-903.

948. Demonty I, Ras RT, van der Knaap HCM, et al. (2009) Continuous dose-response relationship of the LDLcholesterol-lowering effect of phytosterol intake. $J$ Nutr 139, 271-284.

949. Awad AB, Smith AJ \& Fink CS (2001) Plant sterols regulate rat vascular smooth muscle cell growth and prostacyclin release in culture. Prostaglandins Leukot Essent Fatty Acids 64, 323-330.

950. Bouic PJD, Clark A, Lamprecht J, et al. (1999) The effects of B-sitosterol (BSS) and B-sitosterol glucoside (BSSG) mixture on selected immune parameters of marathon runners: inhibition of post marathon immune suppression and inflammation. Int J Sports Med 20, 258-262.

951. Tanaka M, Misawa E, Ito Y, et al. (2006) Identification of five phytosterols from aloe vera gel as anti-diabetic compounds. Biol Pharm Bull 29, 1418-1422.

952. Awad AB, Chen YC, Fink CS, et al. (1996) $\beta$-Sitosterol inhibits HT-29 human colon cancer cell growth and alters membrane lipids. Anticancer Res 16, 2797-2804.

953. Raicht RF, Cohen BI, Fazzini EP, et al. (1980) Protective effect of plant sterols against chemically induced colon tumors in rats. Cancer Res 40, 403-405.

954. Berges RR, Windeler J, Trampisch HJ, et al. (1995) Randomised, placebo-controlled, double-blind clinical trial of $\beta$-sitosterol in patients with benign prostatic hyperplasia. Lancet 345, 1529-1532.

955. Rubis B, Paszel A, Kaczmarek M, et al. (2008) Beneficial or harmful influence of phytosterols on human cells? Br J Nutr 100, 1183-1191. 
956. Awad AB, Roy R \& Fink CS (2003) $\beta$-Sitosterol, a plant sterol, induces apoptosis and activates key caspases in MDA-MB-231 human breast cancer cells. Oncol Rep 10, 497-500.

957. Larner J (2002) D-Chiro-inositol - its functional role in insulin action and its deficit in insulin resistance. Int J Exp Diabetes Res 3, 47-60.

958. Asplin I, Galasko G \& Larner J (1993) Chiro-inositol deficiency and insulin resistance: a comparison of the chiro-inositol- and the myo-inositol-containing insulin mediators isolated from urine, hemodialysate, and muscle of control and type II diabetic subjects. Proc Natl Acad Sci U S A 90, 5924-5928.

959. Brautigan DL, Brown M, Grindrod S, et al. (2005) Allosteric activation of protein phosphatase $2 \mathrm{C}$ by D-chiroinositol-galactosamine, a putative mediator mimetic of insulin action. Biochemistry 44, 11067-11073.

960. Kawa JM, Przybylski R \& Taylor CG (2003) Urinary chiro-inositol and myo-inositol excretion is elevated in the diabetic $\mathrm{db} / \mathrm{db}$ mouse and streptozotocin diabetic rat. Exp Biol Med 228, 907-914.

961. Sun T-H, Heimark DB, Nguygen T, et al. (2002) Both myo-inositol to chiro-inositol epimerase activities and chiro-inositol to myo-inositol ratios are decreased in tissues of GK type 2 diabetic rats compared to Wistar controls. Biochem Biophys Res Commun 293, 1092-1098.

962. Cogram P, Tesh S, Tesh J, et al. (2002) D-Chiro-inositol is more effective than myo-inositol in preventing folateresistant mouse neural tube defects. Hum Reprod 17, 2451-2458.

963. Holub BJ (1986) Metabolism and function of myo-inositol and inositol phospholipids. Annu Rev Nutr 6, 563-597.

964. Katayama T (1997) Effects of dietary myo-inositol or phytic acid on hepatic concentrations of lipids and hepatic activities of lipogenic enzymes in rats fed on corn starch or sucrose. Nutr Res 17, 721-728.

965. Novak JE, Scott Turner R, Agranoff BW, et al. (1999) Differentiated human NT2-N neurons possess a high intracellular content of myo-inositol. J Neurochem $\mathbf{7 2}$ $1431-1440$.

966. Fux M, Levine J, Aviv A, et al. (1996) Inositol treatment of obsessive-compulsive disorder. Am J Psychiatry 153, 1219-1221.

967. Palatnik A, Frolov K, Fux M, et al. (2001) Double-blind, controlled, crossover trial of inositol versus fluvoxamine for the treatment of panic disorder. J Clin Psychopharmacol 21, 335-339.

968. Silver SM, Schroeder BM, Sterns RH, et al. (2006) Myoinositol administration improves survival and reduces myelinolysis after rapid correction of chronic hyponatremia in rats. $J$ Neuropathol Exp Neurol 65, 37-44.

969. Nakanishi T, Turner RJ \& Burg MB (1989) Osmoregulatory changes in $m y o$-inositol transport by renal cells. Proc Natl Acad Sci U S A 86, 6002-6006.

970. Greene DA \& Lattimer SA (1983) Impaired rat sciatic nerve sodium potassium adenosine triphosphatase in acute streptozocin diabetes and its correction by dietary myoinositol supplementation. J Clin Invest 72, 1058-1063.

971. Castano G, Mas R, Fernandez JC, et al. (2001) Effects of policosanol in older patients with type II hypercholesterolemia and high coronary risk. J Gerontol A Biol Sci Med Sci 56, M186-M193.

972. Castaño G, Tula L, Canetti M, et al. (1996) Effects of policosanol in hypertensive patients with type II hypercholesterolemia. Curr Ther Res 57, 691-699.
973. Menendez R, Fernandez SI, Del Rio A, et al. (1994) Policosanol inhibits cholesterol biosynthesis and enhances low density lipoprotein processing in cultured human fibroblasts. Biol Res 27, 199-203.

974. McCarty MF (2002) Policosanol safely down-regulates HMG-CoA reductase - potential as a component of the Esselstyn regimen. Med Hypotheses 59, 268-279.

975. Fraga V, Menendez R, Amor AM, et al. (1997) Effect of policosanol on in vitro and in vivo rat liver microsomal lipid peroxidation. Arch Med Res 28, 355-360.

976. Arruzazabala ML, Molina V, Mas R, et al. (2002) Antiplatelet effects of policosanol (20 and $40 \mathrm{mg} / \mathrm{day})$ in healthy volunteers and dyslipidaemic patients. Clin Exp Pharmacol Physiol 29, 891-897.

977. Carbajal D, Arruzazabala ML, Valdés S, et al. (1998) Effect of policosanol on platelet aggregation and serum levels of arachidonic acid metabolites in healthy volunteers. Prostaglandins Leukot Essent Fatty Acids 58, 61-64.

978. Carbajal D, Molina V, Valdes S, et al. (1995) Antiulcer activity of higher primary alcohols of beeswax. $J$ Pharm Pharmacol 47, 731-733.

979. Saint-John M \& McNaughton L (1986) Octacosanol ingestion and its effects on metabolic responses to submaximal cycle ergometry, reaction time and chest and grip strength. Int Clin Nutr Rev 6, 81-87.

980. Noa M, Más R \& Mesa R (2001) A comparative study of policosanol vs lovastatin on intimal thickening in rabbit cuffed carotid artery. Pharmacol Res 43, 31-37.

981. Ferrari C (2004) Functional foods, herbs and nutraceuticals: towards biochemical mechanisms of healthy aging. Biogerontology 5, 275-289.

982. Reiter R (1995) Oxidative processes and antioxidative defense mechanisms in the aging brain. FASEB J 9, 526-533.

983. Reiter R, Tang L, Garcia JJ, et al. (1997) Pharmacological actions of melatonin in oxygen radical pathophysiology. Life Sci 60, 2255-2271.

984. Sainz RM, Mayo JC, Reiter RJ, et al. (1999) Melatonin regulates glucocorticoid receptor: an answer to its antiapoptotic action in thymus. FASEB $J \mathbf{1 3}, 1547-1556$.

985. Hardeland R, Reiter RJ, Poeggeler B, et al. (1993) The significance of the metabolism of the neurohormone melatonin: antioxidative protection and formation of bioactive substances. Neurosci Biobehav Rev 17, 347-357.

986. Barlow-Walden LR, Reiter RJ, Abe M, et al. (1995) Melatonin stimulates brain glutathione peroxidase activity. Neurochem Int 26, 497-502.

987. Kotler M, Rodríguez C, Sáinz RM, et al. (1998) Melatonin increases gene expression for antioxidant enzymes in rat brain cortex. J Pineal Res 24, 83-89.

988. Zhdanova Wurtm IV, an RJ, Morabito C, et al. (1996) Effects of low oral doses of melatonin, given 2-4 hours before habitual bedtime, on sleep in normal young humans. Sleep 19, 423-431.

989. Reiter RJ, Melchiorri D, Sewerynek E, et al. (1995) A review of the evidence supporting melatonin's role as an antioxidant. $J$ Pineal Res 18, 1-11.

990. Anisimov VN, Zavarzina NY, Zabezhinski MA, et al. (2001) Melatonin increases both life span and tumor incidence in female CBA mice. J Gerontol A Biol Sci Med Sci 56, B311-B323.

991. Srinivasan V, Spence DW, Pandi-Perumal SR, et al. (2008) Therapeutic actions of melatonin in cancer: possible mechanisms. Integr Cancer Ther 7, 189-203.

992. Hearse DJ \& Weber WW (1973) Multiple N-acetyltransferases and drug metabolism. Tissue distribution, characterization and significance of mammalian $N$-acetyltransferase. Biochem J 132, 519-526. 
993. Lindsay RM, McLaren AM \& Baty JD (1988) Effect of hemolysis on the acetylation of $p$-aminobenzoic acid by human whole-blood and washed erythrocytes in vitro. Biochem Soc Trans 16, 1024.

994. Butcher NJ, Ilett KF \& Minchin RF (2000) Substratedependent regulation of human arylamine $\mathrm{N}$-acetyltransferase-1 in cultured cells. Mol Pharmacol 57, 468-473.

995. Failey RB Jr \& Childress RH (1962) The effect of paraaminobenzoic acid on the serum cholesterol level in man. Am J Clin Nutr 10, 158-162.

996. Barbieri B, Stain-Malmgren R \& Papadogiannakis N (1999) $p$-Aminobenzoic acid and its metabolite $p$-acetamidobenzoic acid inhibit agonist-induced aggregation and arachidonic acid-induced $\mathrm{Ca}^{2+}$ (i) transients in human platelets. Thromb Res 95, 235-243.

997. Russell K, Craig ID, Rawlings JM, et al. (2001) The use of $p$-aminobenzoic acid and chromic oxide to confirm complete excreta collection in a carnivore, Felis silvestris catus. Comp Biochem Physiol C Toxicol Pharmacol 130, 339-345.

998. Berger A, Rein D, Schafer A, et al. (2005) Similar cholesterol-lowering properties of rice bran oil, with varied $\gamma$-oryzanol, in mildly hypercholesterolemic men. Eur $J$ Nutr 44, 163-173.

999. Ishihara M, Ito Y, Nakakita T, et al. (1982) Clinical effect of $\gamma$-oryzanol on climacteric disturbance - on serum lipid peroxides (article in Japanese). Nippon Sanka Fujinka Gakkai Zasshi 34, 243-251.

1000. Ishihara M (1984) Effect of $\gamma$-oryzanol on serum lipid peroxide level and clinical symptoms of patients with climacteric disturbances. Asia Oceania J Obstet Gynaecol 10, 317-323.

1001. Itaya K, Kiyonaga J \& Ishikawa M (1976) Studies of $\gamma$-oryzanol (1). Effects on stress-induced ulcer. Nippon Yakurigaku Zasshi 72, 1001-1011.

1002. Parrado J, Miramontes E, Jover M, et al. (2003) Prevention of brain protein and lipid oxidation elicited by a watersoluble oryzanol enzymatic extract derived from rice bran. Eur J Nutr 42, 307-314.

1003. Lee CK, Pugh TD, Klopp RG, et al. (2004) The impact of $\alpha$-lipoic acid, coenzyme $\mathrm{Q}_{10}$, and caloric restriction on life span and gene expression patterns in mice. Free Radic Biol Med 36, 1043-1057.

1004. Eason RC, Archer HE, Akhtar S, et al. (2002) Lipoic acid increases glucose uptake by skeletal muscles of obesediabetic ob/ob mice. Diabetes Obes Metab 4, 29-35.

1005. Khanna S, Roy S, Packer L, et al. (1999) Cytokine-induced glucose uptake in skeletal muscle: redox regulation and the role of $\alpha$-lipoic acid. Am J Physiol Regul Integr Comp Physiol 276, R1327-R1333.

1006. Seetharamaiah GS, Krishnakantha TP \& Chandrasekhara N (1990) Influence of oryzanol on platelet aggregation in rats. J Nutr Sci Vitaminol 36, 291-297.

1007. Cai X-J, Bi X-P, Zhao Z, et al. (2006) The effects of antidepressant treatment on efficacy of antihypertensive therapy in elderly hypertension. Zhonghua Nei Ke Za Zhi 45, 639-641.

1008. Ichimaru Y, Moriyama M, Ichimaru M, et al. (1984) Effects of $\gamma$-oryzanol on gastric lesions and small intestinal propulsive activity in mice. Nippon Yakurigaku Zasshi 84, 537-542.

1009. Jabeen B, Badaruddin M, Ali R, et al. (2007) Attenuation of restraint-induced behavioral deficits and serotonergic responses by stabilized rice bran in rats. Nutr Neurosci $\mathbf{1 0}$, $11-16$.

1010. Nie L, Wise M, Peterson D, et al. (2006) Mechanism by which avenanthramide-c, a polyphenol of oats, blocks cell cycle progression in vascular smooth muscle cells. Free Radic Biol Med 41, 702-708.

1011. Nie L, Wise ML, Peterson DM, et al. (2006) Avenanthramide, a polyphenol from oats, inhibits vascular smooth muscle cell proliferation and enhances nitric oxide production. Atherosclerosis 186, 260-266.

1012. Francis G, Kerem Z, Makkar HPS, et al. (2007) The biological action of saponins in animal systems: a review. Br J Nutr 88, 587-605.

1013. Mimaki Y, Yokosuka A, Kuroda M, et al. (2001) Cytotoxic activities and structure-cytotoxic relationships of steroidal saponins. Biol Pharm Bull 24, 1286-1289.

1014. Potter SM, Jimenez-Flores R, Pollack J, et al. (1993) Protein-saponin interaction and its influence on blood lipids. J Agric Food Chem 41, 1287-1291.

1015. Han L-K, Xu B-J, Kimura Y, et al. (2000) Platycodi radix affects lipid metabolism in mice with high fat diet-induced obesity. J Nutr 130, 2760-2764

1016. Kim YH, Park KH \& Rho HM (1996) Transcriptional activation of the $\mathrm{Cu}, \mathrm{Zn}$-superoxide dismutase gene through the AP2 site by ginsenoside $\mathrm{Rb} 2$ extracted from a medicinal plant, Panax ginseng. J Biol Chem 271, 24539-24543.

1017. Yoshiki Y \& Okubo K (1995) Active oxygen scavenging activity of DDMP (2,3-dihydro-2,5-dihydroxy-6methyl-4H-pyran-4-one) saponin in soybean seed. Biosci Biotechnol Biochem 59, 1556-1557.

1018. Arai S, Osawa T, Ohigashi H, et al. (2001) A mainstay of functional food science in Japan - history, present status, and future outlook. Biosci Biotechnol Biochem 65, 1-3.

1019. Jie L (1995) Pharmacology of oleanolic acid and ursolic acid. J Ethnopharmacol 49, 57-68.

1020. Matsuda H, Li Y, Murakami T, et al. (1999) Structurerelated inhibitory activity of oleanolic acid glycosides on gastric emptying in mice. Bioorg Med Chem 7, 323-327.

1021. Sindambiwe JB, Calomme M, Geerts S, et al. (1998) Evaluation of biological activities of triterpenoid saponins from Maesa lanceolata. J Nat Prod 61, 585-590.

1022. Xi MM, Hai CX, Tang HF, et al. (2008) Antioxidant and antiglycation properties of total saponins extracted from traditional Chinese medicine used to treat diabetes mellitus. Phytother Res 22, 228-237.

1023. Cai J, Liu M, Wang Z, et al. (2002) Apoptosis induced by dioscin in HeLa cells. Biol Pharm Bull 25, 193-196.

1024. Hanausek M, Ganesh P, Walaszek Z, et al. (2001) Avicins, a family of triterpenoid saponins from Acacia victoriae (Bentham), suppress H-ras mutations and aneuploidy in a murine skin carcinogenesis model. Proc Natl Acad Sci U S A 98, 11551-11556.

1025. Liu WK, Xu SX \& Che CT (2000) Anti-proliferative effect of ginseng saponins on human prostate cancer cell line. Life Sci 67, 1297-1306.

1026. Mujoo K, Haridas V, Hoffmann JJ, et al. (2001) Triterpenoid saponins from Acacia victoriae (Bentham) decrease tumor cell proliferation and induce apoptosis. Cancer Res 61, 5486-5490.

1027. Podolak I, Elas M \& Cieszka K (1998) In vitro antifungal and cytotoxic activity of triterpene saponosides and quinoid pigments from Lysimachia vulgaris L. Phytother Res 12, S70-S73.

1028. Zhang YW, Dou DQ, Zhang L, et al. (2001) Effects of ginsenosides from Panax ginseng on cell-to-cell communication function mediated by gap junctions. Planta Med 67, 417-422.

1029. Peng JP, Chen H, Qiao YQ, et al. (1996) Two new steroidal saponins from Allium sativum and their inhibitory effects on blood coagulability (article in Chinese). Yao Xue Xue Bao 31, 607-612. 


\section{Appendix 1}

References cited for evaluating the range (minimum and maximum values) of bioactive compound contents in whole-grain wheat, and wheat bran and germ fractions (data for Tables 1 and 2)*

* All wheat varieties are included, i.e. durum, soft, hard, spring, winter and pigmented wheats; all data are expressed for $100 \mathrm{~g}$ of food. When data are expressed on a DM basis within a reference with no indication of the water content, results are converted on a fresh matter basis considering a mean water content of $13 \%$ for whole-grain wheat, $10 \%$ for wheat bran and $11.4 \%$ for wheat germ (means calculated from US Department of Agriculture database for cereal grains and pasta ${ }^{(479)}$ ).

\section{Whole-grain wheat}

Reduced glutathione: $1 \cdot 04-5 \cdot 74 \mathrm{mg} / 100 \mathrm{~g}^{(210,480)}$

Oxidised glutathione: $0 \cdot 86-2 \cdot 88 \mathrm{mg} / 100 \mathrm{~g}^{(480)}$

Sulfur amino acids:

Methionine: $0 \cdot 17-0 \cdot 24 \mathrm{~g} / 100 \mathrm{~g}^{(479,481-483)}$

Cystine: $0 \cdot 19-0 \cdot 40 \mathrm{~g} / 100 \mathrm{~g}^{(479,482,483)}$

Sugars:

Monosaccharides: $0 \cdot 26-1 \cdot 30 \mathrm{~g} / 100 \mathrm{~g}^{(484,485)}$

Sucrose: $0 \cdot 60-1 \cdot 39 \mathrm{~g} / 100 \mathrm{~g}^{(482,484,485)}$

Total fibre (lignin, oligosaccharides, resistant starch and phytic acid included): $9 \cdot 0-17 \cdot 3 \mathrm{~g} / 100 \mathrm{~g}^{(482,486-492)}$

Insoluble fibre (lignin included): $9 \cdot 5-11.4 \mathrm{~g} / 100 \mathrm{~g}^{(482,488,490,493)}$

Soluble fibre: $1 \cdot 1-3 \cdot 2 \mathrm{~g} / 100 \mathrm{~g}^{(482,488,490,491,493)}$

Cellulose: $2 \cdot 1-2 \cdot 8 \mathrm{~g} / 100 \mathrm{~g}^{(479,482,494)}$

Hemicellulose: $8.6 \mathrm{~g} / 100 \mathrm{~g}^{(485)}$

Lignins: $0 \cdot 9-2 \cdot 8 \mathrm{~g} / 100 \mathrm{~g}^{(485-487)}$

Fructans: $0 \cdot 6-2 \cdot 3 \mathrm{~g} / 100 \mathrm{~g}^{(485,487,495-497)}$

Raffinose: $0 \cdot 13-0 \cdot 59 \mathrm{~g} / 100 \mathrm{~g}^{(482,484,485,495,496)}$

Stachyose: $0 \cdot 05-0 \cdot 17 \mathrm{~g} / 100 \mathrm{~g}^{(484,485)}$

Total arabinoxylans: $1 \cdot 2-6 \cdot 8 \mathrm{~g} / 100 \mathrm{~g}^{(482,486,487,491,498,499)}$

Water-extractable arabinoxylans: $0 \cdot 2-1 \cdot 2 \mathrm{~g} / 100 \mathrm{~g}^{(486,491)}$

$\beta$-Glucans: $0 \cdot 2-4 \cdot 7 \mathrm{~g} / 100 \mathrm{~g}^{(485,486,491,492,498,500)}$

Phytic acid: $0 \cdot 28-1.50 \mathrm{~g} / 100 \mathrm{~g}^{(482,501-506)}$

Fe: $1 \cdot 0-14 \cdot 2 \mathrm{mg} / 100 \mathrm{~g}^{(426,427,479,482,483,502,504,507-511)}$

$\mathrm{Mg}$ : $17-191 \mathrm{mg} / 100 \mathrm{~g}^{(479,482,483,504,507,511,512)}$

Zn: $0 \cdot 8-8 \cdot 9 \mathrm{mg} / 100 \mathrm{~g}^{(426,427,479,482,483,504,507,509,511-513)}$

Mn: $0 \cdot 9-7 \cdot 8 \mathrm{mg} / 100 \mathrm{~g}^{(479,482,483,504,507,509,511,512)}$

Cu: $0 \cdot 09-1 \cdot 21 \mathrm{mg} / 100 \mathrm{~g}^{(479,482,483,504,507,509,511-513)}$

Se: $0 \cdot 0003-3 \cdot 0000 \mathrm{mg} / 100 \mathrm{~g}^{(479,482,483,507,512,514,515)}$

P: $218-792 \mathrm{mg} / 100 \mathrm{~g}^{(479,482,483,507,511)}$

Ca: $7-70 \mathrm{mg} / 100 \mathrm{~g}^{(479,482,483,504,507,511)}$

Na: $2-16 \mathrm{mg} / 100 \mathrm{~g}^{(479,482,483)}$

$\mathrm{K}: 209-635 \mathrm{mg} / 100 \mathrm{~g}^{(479,482,483,504,507,511)}$

Thiamin (vitamin $\mathrm{B}_{1}$ ): 0.13-0.99 mg/100 $\mathrm{g}^{(314,479,482,483,516-519)}$

Riboflavin (vitamin $\mathrm{B}_{2}$ ): 0.04-0.31 mg/100 g(314,479,482,483,516-518)

Niacin (vitamin $\mathrm{B}_{3}$ ): $1 \cdot 9-11 \cdot 1 \mathrm{mg} / 100 \mathrm{~g}^{(314,479,482,483,517,518)}$

Pantothenic acid (vitamin $\mathrm{B}_{5}$ ): $072-1.99 \mathrm{mg} / 100 \mathrm{~g}^{(314,479,482,483,518)}$
Pyridoxine (vitamin $\mathrm{B}_{6}$ ): 0.09-0.66 mg/100 g $\mathrm{g}^{(314,479,482,483,516-518)}$

Biotin (vitamin $\mathrm{B}_{8}$ ): 0.002-0.011 mg/100 $\mathrm{g}^{(314,482)}$

Folates (vitamin $\mathrm{B}_{9}$ ): 0.014-0.087 mg/100 $\mathrm{g}^{(314,482,518,520-522)}$

Tocols (vitamin E) = tocopherols + tocotrienols: $2 \cdot 3-7 \cdot 1 \mathrm{mg} / 100 \mathrm{~g}^{(482,492,523-526)}$

Total tocopherols: $1 \cdot 06-2 \cdot 89 \mathrm{mg} / 100 \mathrm{~g}^{(482,523-526)}$ $\alpha$-Tocopherol: $0.34-3.49 \mathrm{mg} / 100 \mathrm{~g}^{(479,482,483,518,523-527)}$

Total tocotrienols: $1.09-4.49 \mathrm{mg} / 100 \mathrm{~g}^{(482,523-526)}$

Phylloquinone (vitamin K): 0.002-0.020 mg/100 g ${ }^{(479,482)}$

Total carotenoids: $0 \cdot 044-0 \cdot 626 \mathrm{mg} / 100 \mathrm{~g}^{(479,493,528-530)}$

$\beta$-Carotene: $0 \cdot 005-0.025 \mathrm{mg} / 100 \mathrm{~g}^{(479,482,527,528)}$

Lutein: $0 \cdot 026-0.383 \mathrm{mg} / 100 \mathrm{~g}^{(14,527-531)}$

Zeaxanthin: $0 \cdot 009-0.039 \mathrm{mg} / 100 \mathrm{~g}^{(14,527,529-531)}$

$\beta$-Cryptoxanthin: $1 \cdot 12-13 \cdot 28 \mu \mathrm{g} / 100 \mathrm{~g}^{(14)}$

Total phenolic acids: $16-102 \mathrm{mg} / 100 \mathrm{~g}^{(197,492)}$

Extractable (free and conjugated) phenolic acids: $5-39 \mathrm{mg} / 100 \mathrm{~g}^{(197,492)}$

Bound phenolic acids: $14-78 \mathrm{mg} / 100 \mathrm{~g}^{(197,492)}$

Total ferulic acid: $16-213 \mathrm{mg} / 100 \mathrm{~g}^{(197,499,527,532-535)}$

Free/soluble-conjugated ferulic acid: $0.7-4.9 \mathrm{mg} / 100 \mathrm{~g}^{(527,532)}$

Bound ferulic acid: $14-64 \mathrm{mg} / 100 \mathrm{~g}^{(197,527,532)}$

Total dehydrodiferulic acid: $1 \cdot 5-76 \cdot 0 \mathrm{mg} / 100 \mathrm{~g}^{(197,533,534)}$

Total dehydrotrimer ferulic acid: $2 \cdot 6-3 \cdot 5 \mathrm{mg} / 100 \mathrm{~g}^{(501,533)}$

Total flavonoids: $30-43 \mathrm{mg}$ catechin equivalents/100 $\mathrm{g}^{(14,532)}$

Free flavonoids: $2 \cdot 15-4.86 \mathrm{mg}$ catechin equivalents $/ 100 \mathrm{~g}^{(14,532)}$

Bound flavonoids: $28-40 \mathrm{mg}$ catechin equivalents/100 $\mathrm{g}^{(14,532)}$

Anthocyanins: $0 \cdot 45-52.60 \mathrm{mg} / 100 \mathrm{~g}^{(308,493,536,537)}$

Isoflavonoids:

Daidzein: $2 \cdot 1 \mu \mathrm{g} / 100 \mathrm{~g}^{(538)}$

Genistein: $12.7 \mu \mathrm{g} / 100 \mathrm{~g}^{(538)}$

Lignans: 0.199-0.619 mg/100 $\mathrm{g}^{(293,539,540)}$

Alkylresorcinols: $11 \cdot 6-128.8 \mathrm{mg} / 100 \mathrm{~g}^{(392,393,396,399,492,501,541)}$

Betaine: $22-291 \mathrm{mg} / 100 \mathrm{~g}^{(227,483,542)}$

Total choline: $27-195 \mathrm{mg} / 100 \mathrm{~g}^{(227,313,314,483,542)}$

Phytosterols: $57-98 \mathrm{mg} / 100 \mathrm{~g}^{(482,489,492,543-546)}$

Total D-chiro-inositol: $17 \mathrm{mg} / 100 \mathrm{~g}^{(245)}$

Policosanol: $0 \cdot 30-5 \cdot 62 \mathrm{mg} / 100 \mathrm{~g}^{(547)}$

Melatonin: $0 \cdot 2-0 \cdot 4 \mu \mathrm{g} / 100 \mathrm{~g}^{(308)}$

p-Aminobenzoic acid (PABA): $0 \cdot 34-0.55 \mathrm{mg} / 100 \mathrm{~g}^{(313,314)}$

\section{Wheat bran}

$\alpha$-Linolenic acid (18:3n-3): 0.16 g/100 $\mathrm{g}^{(548)}$

Reduced glutathione: about $1.7-19.4 \mathrm{mg} / 100 \mathrm{~g}^{(549)}$

Oxidised glutathione: about $6 \cdot 1-21.4 \mathrm{mg} / 100 \mathrm{~g}^{(549)}$

Sulfur amino acids:

Methionine: $0 \cdot 20-0 \cdot 29 \mathrm{~g} / 100 \mathrm{~g}^{(479,482,483)}$

Cystine: $0 \cdot 32-0 \cdot 45 \mathrm{~g} / 100 \mathrm{~g}^{(479,482,483)}$

Sugars:

Monosaccharides: $0 \cdot 14-0 \cdot 63 \mathrm{~g} / 100 \mathrm{~g}^{(482,485)}$

Sucrose: $1 \cdot 8-3 \cdot 4 \mathrm{~g} / 100 \mathrm{~g}^{(482,485,550,551)}$ 
Total fibre (lignin, oligosaccharides, resistant starch and phytic acid included):

$35 \cdot 7-52 \cdot 8 \mathrm{~g} / 100 \mathrm{~g}^{(221,471,482,487-489,552-554)}$

Insoluble fibre (lignin included):

$32 \cdot 4-41 \cdot 6 \mathrm{~g} / 100 \mathrm{~g}^{(482,485,488,493,552,555-557)}$

Soluble fibre: $1 \cdot 3-5 \cdot 8 \mathrm{~g} / 100 \mathrm{~g}^{(482,485,488,493,552,555)}$

Cellulose: $6 \cdot 5-9 \cdot 9 \mathrm{~g} / 100 \mathrm{~g}^{(471,479,482,485,556,558-561)}$

Hemicellulose: $20 \cdot 8-33 \cdot 0 \mathrm{~g} / 100 \mathrm{~g}^{(485,550,558-561)}$

Lignins: $2 \cdot 2-9 \mathrm{~g} / 100 \mathrm{~g}^{(221,485,487,552,556,558-562)}$

Fructans: $0 \cdot 6-4 \cdot 0 \mathrm{~g} / 100 \mathrm{~g}^{(485,487,551)}$

Raffinose: $1 \cdot 08-1 \cdot 32 \mathrm{~g} / 100 \mathrm{~g}^{(482,485,550,551)}$

Stachyose: $0 \cdot 04-0 \cdot 36 \mathrm{~g} / 100 \mathrm{~g}^{(482,485,551)}$

Total arabinoxylans: $5 \cdot 0-26 \cdot 9 \mathrm{~g} / 100 \mathrm{~g}^{(471,486,487,492,554,562,563)}$

Water-extractable arabinoxylans: $0.1-1 \cdot 4 \mathrm{~g} / 100 \mathrm{~g}^{(486,492,562,563)}$

$\beta$-Glucans: $1 \cdot 1-2 \cdot 6 \mathrm{~g} / 100 \mathrm{~g}^{(471,485,562)}$

Phytic acid: $2 \cdot 3-6 \cdot 0 \mathrm{~g} / 100 \mathrm{~g}^{(471,482,505,553,556,557,559,564-566)}$

Fe: $2 \cdot 5-19 \cdot 0 \mathrm{mg} / 100 \mathrm{~g}^{(70,479,482,483,510,511,556,557,567)}$

$\mathrm{Mg}: 390-640 \mathrm{mg} / 100 \mathrm{~g}^{(70,479,482,483,511,559,568)}$

Zn: $2 \cdot 5-14 \cdot 1 \mathrm{mg} / 100 \mathrm{~g}^{(70,479,482,483,510,511,557,559,567,568)}$

Mn: $4-14 \mathrm{mg} / 100 \mathrm{~g}^{(70,479,482,483,511)}$

Cu: $0 \cdot 84-2 \cdot 20 \mathrm{mg} / 100 \mathrm{~g}^{(70,479,482,483,511)}$

Se: $2-78 \mu \mathrm{g} / 100 \mathrm{~g}^{(479,482,483)}$

P: $900-1500 \mathrm{mg} / 100 \mathrm{~g}^{(70,479,482,483,511,559,568)}$

Ca: $24-150 \mathrm{mg} / 100 \mathrm{~g}^{(70,479,482,483,511,559,568)}$

Na: $2-41 \mathrm{mg} / 100 \mathrm{~g}^{(70,479,482,483)}$

$\mathrm{K}: 1182-1900 \mathrm{mg} / 100 \mathrm{~g}^{(70,479,482,483,511)}$

Thiamin (vitamin $\mathrm{B}_{1}$ ): $0 \cdot 506-0 \cdot 800 \mathrm{mg} / 100 \mathrm{~g}^{(314,479,482,483)}$

Riboflavin (vitamin $\mathrm{B}_{2}$ ): 0.210-0.800 mg/100 $\mathrm{g}^{(314,479,482,483)}$

Niacin (vitamin $\mathrm{B}_{3}$ ): $13 \cdot 6-35 \cdot 9 \mathrm{mg} / 100 \mathrm{~g}^{(314,479,482,483)}$

Pantothenic acid (vitamin $\mathrm{B}_{5}$ ): $2 \cdot 2-4 \cdot 1 \mathrm{mg} / 100 \mathrm{~g}^{(314,479,482,483)}$

Pyridoxine (vitamin $\mathrm{B}_{6}$ ): $0 \cdot 704-1 \cdot 303 \mathrm{mg} / 100 \mathrm{~g}^{(479,482,483,569)}$

Biotin (vitamin $\mathrm{B}_{8}$ ): 0.044 mg/100 $\mathrm{g}^{(314,482)}$

Folates (vitamin $\mathrm{B}_{9}$ ): $0 \cdot 088-0 \cdot 373 \mathrm{mg} / 100 \mathrm{~g}^{(314,482,521,570)}$

Tocols $($ vitamin $\mathrm{E})=$ tocopherols + tocotrienols: $9.5 \mathrm{mg} / 100 \mathrm{~g}^{(482)}$

Total tocopherols: $2.4 \mathrm{mg} / 100 \mathrm{~g}^{(482)}$

$\alpha$-Tocopherol: $0 \cdot 13-2 \cdot 84 \mathrm{mg} / 100 \mathrm{~g}^{(479,482,483,571,572)}$

Total tocotrienols: $7 \cdot 1 \mathrm{mg} / 100 \mathrm{~g}^{(482)}$

Phylloquinone (vitamin K): $0 \cdot 002-0 \cdot 083 \mathrm{mg} / 100 \mathrm{~g}^{(479,482)}$

Total carotenoids: $0 \cdot 25-1 \cdot 18 \mathrm{mg} / 100 \mathrm{~g}^{(479,493)}$

$\beta$-Carotene: $0 \cdot 003-0 \cdot 010 \mathrm{mg} / 100 \mathrm{~g}^{(479,482,572)}$

Lutein: $0 \cdot 050-0 \cdot 180 \mathrm{mg} / 100 \mathrm{~g}^{(571,572)}$

Zeaxanthin: 0.025-0.219 mg/100 $\mathrm{g}^{(571,572)}$

ß-Cryptoxanthin: $0.018-0.064 \mathrm{mg} / 100 \mathrm{~g}^{(571,572)}$

Total phenolic acids: $761-1384 \mathrm{mg} / 100 \mathrm{~g}^{(533,573)}$

Extractable (free and conjugated) phenolic acids: $46-63 \mathrm{mg}$ gallic acid equivalents/100 $\mathrm{g}^{(574,575)}$

Bound phenolic acids: $148-340 \mathrm{mg}$ gallic acid equivalents/100 $\mathrm{g}^{(574,575)}$

Total ferulic acid: $138-631 \mathrm{mg} / 100 \mathrm{~g}^{(154,194,263,264,365,499,533,573,575,576)}$

Free/soluble-conjugated ferulic acid: $1 \cdot 34-23.05 \mathrm{mg} / 100 \mathrm{~g}^{(154,194,571,572,574,575,577-579)}$
Bound ferulic acid: $122-286 \mathrm{mg} / 100 \mathrm{~g}^{(154,574,575)}$

Total dehydrodiferulic acid: $13-230 \mathrm{mg} / 100 \mathrm{~g}^{(194,533,534,573)}$

Total dehydrotrimer ferulic acid: $15-25 \mathrm{mg} / 100 \mathrm{~g}^{(533)}$

Total flavonoids: $14 \cdot 9-40 \cdot 6 \mathrm{mg} / 100 \mathrm{~g}^{(193)}$

Anthocyanins: $0 \cdot 9-48 \cdot 0 \mathrm{mg} / 100 \mathrm{~g}^{(493,536,537,580)}$

Isoflavonoids:

Daidzein: $3.5 \mu \mathrm{g} / 100 \mathrm{~g}^{(293)}$

Genistein: $3 \cdot 8-6 \cdot 9 \mu \mathrm{g} / 100 \mathrm{~g}^{(293,538)}$

Lignans: $2 \cdot 8-6 \cdot 7 \mathrm{mg} / 100 \mathrm{~g}^{(221,581)}$

Alkylresorcinols: $215-323 \mathrm{mg} / 100 \mathrm{~g}^{(365,392,582)}$

Betaine: $230-1506 \mathrm{mg} / 100 \mathrm{~g}^{(227,477,483,583,584)}$

Total choline: $74-270 \mathrm{mg} / 100 \mathrm{~g}^{(227,314,477,483,583)}$

Phytosterols: $121-195 \mathrm{mg} / 100 \mathrm{~g}^{(482,543,546,585)}$

Total D-chiro-inositol: not detected ${ }^{(245)}$

Policosanol: $0 \cdot 11-3 \cdot 00 \mathrm{mg} / 100 \mathrm{~g}^{(574,586)}$

PABA: $1.34 \mathrm{mg} / 100 \mathrm{~g}^{(314)}$

\section{Wheat germ}

$\alpha$-Linolenic acid (18:3n-3): 0.47-0.59 mg/100 g ${ }^{(25,548,587)}$

Reduced glutathione: about $19 \cdot 4-245 \cdot 7 \mathrm{mg} / 100 \mathrm{~g}^{(549)}$

Oxidised glutathione: about $15 \cdot 3-122 \cdot 4 \mathrm{mg} / 100 \mathrm{~g}^{(549)}$

Sulfur amino acids:

Methionine: $0 \cdot 39-0 \cdot 58 \mathrm{~g} / 100 \mathrm{~g}^{(479,482,483)}$

Cystine: $0 \cdot 35-0 \cdot 61 \mathrm{~g} / 100 \mathrm{~g}^{(479,482,483)}$

Sugars:

Glucose: $<390-700 \mathrm{mg} / 100 \mathrm{~g}^{(482,588-590)}$

Fructose: $<200-801 \mathrm{mg} / 100 \mathrm{~g}^{(482,588-590)}$

Sucrose: $7 \cdot 7-16 \cdot 0 \mathrm{~g} / 100 \mathrm{~g}^{(482,550,588-592)}$

Total fibre (lignins, oligosaccharides, resistant starch and phytic acid included): $10 \cdot 6-24 \cdot 7 \mathrm{~g} / 100 \mathrm{~g}^{(482,487-489)}$

Insoluble fibre: $8 \cdot 5-18 \cdot 6 \mathrm{~g} / 100 \mathrm{~g}^{(25,482,488)}$

Soluble fibre: $2 \cdot 1-6 \cdot 1 \mathrm{~g} / 100 \mathrm{~g}^{(25,482,488)}$

Cellulose: $7 \cdot 5 \mathrm{~g} / 100 \mathrm{~g}^{(550)}$

Hemicellulose: $6 \cdot 8 \mathrm{~g} / 100 \mathrm{~g}^{(550)}$

Lignins: $1.3-1.6 \mathrm{~g} / 100 \mathrm{~g}^{(487}$

Fructans: $1 \cdot 7-2 \cdot 5 \mathrm{~g} / 100 \mathrm{~g}^{(487)}$

Raffinose: $5 \cdot 0-10 \cdot 9 \mathrm{~g} / 100 \mathrm{~g}^{(550,588-592)}$

Total arabinoxylans: $5 \cdot 6-9 \cdot 1 \mathrm{~g} / 100 \mathrm{~g}^{(487,563)}$

Water-extractable arabinoxylans: $0 \cdot 37 \mathrm{~g} / 100 \mathrm{~g}^{(563)}$

Phytic acid: $1 \cdot 3-2 \cdot 2 \mathrm{~g} / 100 \mathrm{~g}^{(482,565,593)}$

Fe: $3 \cdot 9-10 \cdot 3 \mathrm{mg} / 100 \mathrm{~g}^{(479,482,483,589,594)}$

Mg: $200-340 \mathrm{mg} / 100 \mathrm{~g}^{(479,482,483,589,594)}$

Zn: $10-18 \mathrm{mg} / 100 \mathrm{~g}^{(479,482,483,589,594)}$

Mn: $9-18 \mathrm{mg} / 100 \mathrm{~g}^{(479,482,483,594)}$

Cu: $0 \cdot 70-1 \cdot 42 \mathrm{mg} / 100 \mathrm{~g}^{(479,482,483,589,594)}$

Se: $1-79 \mu \mathrm{g} / 100 \mathrm{~g}^{(479,482,483)}$

P: $770-1337 \mathrm{mg} / 100 \mathrm{~g}^{(479,482,483,589,594)}$

Ca: $36-84 \mathrm{mg} / 100 \mathrm{~g}^{(479,482,483,589,594)}$

Na: $2-37 \mathrm{mg} / 100 \mathrm{~g}^{(479,482,483,589,594)}$

K: $842-1300 \mathrm{mg} / 100 \mathrm{~g}^{(479,482,483,589,594)}$

Thiamin (vitamin $\mathrm{B}_{1}$ ): $0 \cdot 8-2 \cdot 7 \mathrm{mg} / 100 \mathrm{~g}^{(314,479,482,483)}$

Riboflavin (vitamin $\mathrm{B}_{2}$ ): 0.49-0.80 mg/100 $\mathrm{g}^{(314,479,482,483)}$ 
Niacin (vitamin $\left.\mathrm{B}_{3}\right): 4 \cdot 0-8 \cdot 5 \mathrm{mg} / 100 \mathrm{~g}^{(314,479,482,483)}$

Pantothenic acid (vitamin $\left.\mathrm{B}_{5}\right): 1-2 \cdot 7 \mathrm{mg} / 100 \mathrm{~g}^{(314,479,482,483)}$

Pyridoxine (vitamin $\mathrm{B}_{6}$ ): $0.49-1.98 \mathrm{mg} / 100 \mathrm{~g}^{(479,482,483)}$

Biotin (vitamin $\mathrm{B}_{8}$ ): $17 \cdot 0-17 \cdot 4 \mu \mathrm{g} / 100 \mathrm{~g}^{(314,482)}$

Folates (vitamin $\mathrm{B}_{9}$ ): $0 \cdot 14-0 \cdot 70 \mathrm{mg} / 100 \mathrm{~g}^{(314,482,483,521)}$

Tocols $($ vitamin $\mathrm{E})=$ tocopherols + tocotrienols: $23 \cdot 1-31 \mathrm{mg} / 100 \mathrm{~g}^{(482,524)}$

Total tocopherols: $21 \cdot 5-30 \cdot 6 \mathrm{mg} / 100 \mathrm{~g}^{(482,524)}$ $\alpha$-Tocopherol: $3 \cdot 1-22 \mathrm{mg} / 100 \mathrm{~g}^{(482,483,524,525,591)}$

Total tocotrienols: $1 \cdot 3-1.6 \mathrm{mg} / 100 \mathrm{~g}^{(482,524)}$

Phylloquinone (vitamin K): 0.003-0.350 mg/100 g ${ }^{(482)}$ $\beta$-Carotene: $0.062 \mathrm{mg} / 100 \mathrm{~g}^{(482)}$

Extractable (free and conjugated) phenolic acids: about $51 \mathrm{mg} / 100 \mathrm{~g}^{(194)}$

Total ferulic acid: $7-124 \mathrm{mg} / 100 \mathrm{~g}^{(194,499)}$

Free/conjugated soluble ferulic acid: about $18 \mathrm{mg} / 100 \mathrm{~g}^{(194)}$

Total dehydrodiferulic acid: about $9 \mathrm{mg} / 100 \mathrm{~g}^{(194)}$

Total flavonoids: $300 \mathrm{mg}$ rutin equivalents/100 $\mathrm{g}^{(595)}$

Lignans: $0.490 \mathrm{mg} / 100 \mathrm{~g}^{(596)}$

Betaine: $306-1395 \mathrm{mg} / 100 \mathrm{~g}^{(227,477,483)}$

$1395 \mathrm{mg} / 100 \mathrm{~g}^{(477)}$ : toasted

Total choline: $152-330 \mathrm{mg} / 100 \mathrm{~g}^{(227,314,483)}$

$152 \mathrm{mg} / 100 \mathrm{~g}^{(477)}$ : toasted

Phytosterols: $410-450 \mathrm{mg} / 100 \mathrm{~g}^{(489,546,597)}$

Policosanol: $1.0 \mathrm{mg} / 100 \mathrm{~g}^{(586)}$

PABA: $0.852 \mathrm{mg} / 100 \mathrm{~g}^{(314)}$

\section{Appendix 2}

References for evaluating the range of compound bioavailability and degree of fibre-type compounds fermentation from whole-grain wheat, wheat bran and/or derived products (data for Table 2).

\section{Whole-grain wheat and derived products}

Reduced glutathione: negligible in humans as free compound ${ }^{(209)}$ Stachyose and raffinose:

Completely fermented in vitro within $48 \mathrm{~h}$ as free compound ${ }^{(296)}$ $97-99 \%$ in $\operatorname{dogs}(598)$

Total fibre: $34 \%$ in human subjects fed wholemeal bread ${ }^{(599)}$ Cellulose: $20 \%$ in human subjects fed wholemeal bread ${ }^{(599)}$ Hemicellulose: $46 \%$ in human subjects fed wholemeal bread $^{(599)}$

Lignins: $4 \%$ in human subjects fed wholemeal bread ${ }^{(599)}$ Phytic acid: 54-79\% apparently degraded (faeces recovery) in human subjects fed Hovis bread (whole bread) ${ }^{(600)}$

Rapidly and almost fully absorbed (about $79 \%$ ) in upper part of the gastrointestinal tract of rats fed free compound ${ }^{(601)}$

Small-intestinal phytases have high activity in rats and very much lower activity in human subjects and pigs ${ }^{(217)}$

Fe: $1-20 \%$ in human subjects fed usual diets ${ }^{(204)}$
$\mathrm{Mg}$ :

$70 \%$ in rats fed whole-wheat flour ${ }^{(219)}$

$21-28 \%$ in human subjects fed brown bread diet ${ }^{(602)}$

$50 \%$ in human subjects fed a typical diet ${ }^{(603)}$

$57.6 \%$ in human subjects fed a standard $\operatorname{diet}^{(604)}$

$\mathrm{Zn}$ :

$16.6 \%$ in human subjects consuming wholemeal bread ${ }^{(605)}$

$20 \%$ in adult women consuming whole-wheat tortillas ${ }^{(606)}$

$35 \%$ in rats fed whole-wheat flour ${ }^{(219)}$

$88.9-94.6 \%$ in rats fed whole-wheat flour ${ }^{(607)}$

$18.5 \%$ in rats fed wheatmeal ${ }^{(608)}$

$60-82 \%$ in rats fed whole-grain wheat ${ }^{(609)}$

$30-37 \%$ in rats fed whole-wheat flour chapatti ${ }^{(610)}$

$\mathrm{Cu}$ :

$62-85 \%$ in human subjects fed whole-wheat bread ${ }^{(611)}$

$16 \cdot 3-16 \cdot 5 \%$ in rats fed free compound ${ }^{(71,73)}$

Se:

$81 \cdot 1-84.5 \%$ in rats fed whole-wheat flour ${ }^{(607)}$

$73-86 \%$ in rats fed whole wheat as compared with sodium selenite ${ }^{(612)}$

$100 \%$ in rats fed whole-wheat flour as compared with sodium selenite ${ }^{(613)}$

P: $41-55 \%$ in human subjects fed brown bread diet ${ }^{(602)}$

Ca:

$81.7 \%$ in human subjects fed whole-wheat bread ${ }^{(614)}$

$43-44 \%$ in rats fed whole-wheat flour chapatti ${ }^{(610)}$

$85.7-92.8 \%$ in rats fed whole-wheat flour ${ }^{(607)}$

Thiamin (vitamin $\mathrm{B}_{1}$ ): $91 \%$ in rats fed whole-wheat bread compared with free thiamine mononitrate $(100 \%)^{(519)}$

Riboflavin (vitamin $\mathrm{B}_{2}$ ): $95 \%$ as oral supplement in human subjects $^{(615)}$

Niacin (vitamin $\mathrm{B}_{3}$ ): low ${ }^{(19)}$

Pantothenic acid (vitamin $\mathrm{B}_{5}$ ): about $50 \%$ in human subjects for average American diet ${ }^{(616)}$

Pyridoxine (vitamin $\mathrm{B}_{6}$ ): $71-79 \%$ for an average American diet compared with free compound ${ }^{(616)}$

$\alpha$-Tocopherol: $70 \%$ in human subjects fed free compound $^{(617)}$

Total ferulic acid: $3 \cdot 2-3 \cdot 6 \%$ urinary excretion in rats ${ }^{(152)}$

Free/soluble-conjugated ferulic acid: at least that of wheat bran in rat small intestine ${ }^{(154)}$

Bound ferulic acid: a small fraction released within small intestine by intestinal esterases ${ }^{(618)}$

Alkylresorcinols: $60-79 \%$ from ileal samples in pigs fed whole-grain rye bread ${ }^{(619)}$

Phytosterols: weakly absorbed from the gut ${ }^{(620)}$

Total free inositols (myo- and chiro-inositol):

Apparently high in rats fed free compounds for myo-inositol ${ }^{(256)}$

Apparently high in women fed free compounds for chiro-inositol $^{(621)}$

Apparently high in old human subjects fed free compounds for pinitol ${ }^{(62)}$ 


\section{Wheat bran}

Total fibre:

$55.6 \%$ neutral sugars in human subjects fed wheat bran ${ }^{(552)}$ $34 \%$ neutral sugars in human subjects fed wheat bran ${ }^{(623)}$

$35-42 \%$ neutral-detergent fibre in human subjects fed coarse and fine bran ${ }^{(561)}$

36.9 and $41.1 \%$ in rats fed coarse and fine brans ${ }^{(624)}$

$39 \%$ in rats fed wheat bran ${ }^{(623)}$

$49.1 \%$ NSP in rats fed wheat bran ${ }^{(625)}$

$58.8-65.0 \%$ in pigs fed coarse and fine bran cell walls ${ }^{(626)}$ $41.5 \%$ in pigs fed wheat bran-based diet ${ }^{(627)}$

Insoluble fibre:

$42.3 \%$ in rats fed wheat bran ${ }^{(625)}$

Cellulose:

$6-23 \%$ in human subjects fed coarse and fine bran ${ }^{(561)}$

$7 \%$ in human subjects fed wheat bran ${ }^{(623)}$

$13 \cdot 8-21.9 \%$ in rats fed coarse and fine brans ${ }^{(624)}$

$24.1 \%$ in pigs fed wheat bran-based diet ${ }^{(627)}$

$18.2-23.7 \%$ in pigs fed coarse and fine brans ${ }^{(626)}$

Hemicellulose:

$50-54 \%$ in human subjects fed coarse and fine brans ${ }^{(561)}$

$69.4-74.4 \%$ in pigs fed coarse and fine brans ${ }^{(626)}$

$46.5 \%$ non-cellulosic neutral sugar residues in pigs fed wheat bran-based diet ${ }^{(627)}$

\section{Lignins:}

Undigested in humans ${ }^{(561)}$

$0 \%$ in rats fed wheat bran ${ }^{(623)}$

$0-4 \%$ in rats fed processed wheat bran ${ }^{(628)}$

Soluble fibre: $72.9 \%$ in rats fed wheat bran fibre ${ }^{(625)}$

Total arabinoxylans: $49.2 \%$ arabinose and $71.1 \%$ xylose in human subjects fed wheat bran ${ }^{(552)}$

Phytic acid:

Phytate from wheat bran without phytase is almost not absorbed at the intestinal level in humans ${ }^{(629)}$

$58-60 \%$ degraded into lower myo-inositol phosphates in ileostomates fed raw wheat bran ${ }^{(629,630)}$ and only $5 \%$ with phytase-deactivated wheat bran ${ }^{(629,630)}$

$58 \%$ degraded in ileostomates and $25 \%$ hydrolysed for extruded wheat bran (loss of phytase activity) ${ }^{(630)}$

$\mathrm{Fe}:$

$3.8 \%$ in human subjects fed rolls made of wheat bran and white wheat flour ${ }^{(631)}$

Negative effect of bran on Fe absorption is not observed in rats ${ }^{(632)}$

Se:

About $60 \%$ in rats fed wheat bran compared with sodium selenite and selenomethionine biological value ${ }^{(633)}$

$80 \%$ in rats fed wheat bran as compared with sodium selenite ${ }^{(613)}$

P: $41-56 \%$ in human subjects fed sodium phytate + white bread $^{(602)}$

Ca: $22.3 \%$ in human subjects fed extruded wheat bran cereals $^{(614)}$
Niacin (vitamin $\mathrm{B}_{3}$ ):

$27-38 \%$ in human subjects fed a concentrate of bound niacin from wheat bran ${ }^{(634)}$

$17 \%$ in rats fed a concentrate of bound niacin from wheat bran (cited in Carter \& Carpenter ${ }^{(634)}$ )

Pyridoxine (vitamin $\mathrm{B}_{6}$ ): unavailable in human subjects fed wheat bran ${ }^{(635)}$

Folates (vitamin $\mathrm{B}_{9}$ ): low in human subjects fed wheat bran $^{(467)}$

Tocopherols/tocotrienols (vitamin E): not available in rats fed wheat bran ${ }^{(636)}$

Bound phenolic acids:

$32.7 \%$ in pigs fed a wheat bran diet ${ }^{(627)}$

Partially and slowly solubilised from wheat bran within a human model colon ${ }^{(264)}$

Total ferulic acid:

$<5 \%$ in small intestine of rats fed wheat bran-based $\operatorname{diet}^{(154)}$

$3.9 \%$ urinary excretion in rats fed wheat $\operatorname{bran}^{(152)}$

$1.99-5.65 \%$ urinary excretion in human subjects fed highbran cereal $^{(196)}$

Free/soluble-conjugated ferulic acid:

High in rat small intestine fed wheat bran ${ }^{(154)}$

$27.77-78.92 \%$ urinary excretion in human subjects fed high-bran cereal $^{(196)}$

Bound ferulic acid: a small fraction (\%?) released within rat small intestine by intestinal esterases following wheat bran consumption ${ }^{(618)}$

Dehydrodiferulic acid:

Undetectable in plasma of human subjects fed high-bran cereal $^{(196)}$

Free diferulic acid can be absorbed from the gut in rats fed wheat bran ${ }^{(637)}$

Alkylresorcinols: $45-71 \%$ from ileostomy effluents in human subjects fed rye bran soft/crisp bread ${ }^{(393)}$

Phytosterols: weakly absorbed from the gut in human subjects ${ }^{(620)}$

\section{Appendix 3}

References for the physiological mechanisms and health effects of bioactive compounds from whole-grain wheat, and wheat bran and germ fractions (data for Tables 3 and 4)*

* Keywords relative to the physiological mechanisms involved, health outcomes associated with bioactive compounds and the corresponding reference(s) are given; the models used, i.e. human, animals or in vitro cultured cells, may be found in references cited.

$\alpha$-Linolenic acid (18:3n-3):

Health and diseases ${ }^{(638)}$; CVD $^{(548,638-641)}$; anti-atherosclerotic $^{(298)}$; depression and anxiety ${ }^{(642,643)}$; plasma $\mathrm{TAG}^{(644)}$; blood clotting, thrombosis, plasma lipid profile, blood pressure and inflammation ${ }^{(638)}$; $\operatorname{colon}^{(645)}$ and breast ${ }^{(646)}$ cancers; synthesis of cytokines and mitogens $^{(638)}$; arachidonic acid $(20: 4 n-6)$ and eicosanoids in tissues (such as lung) and plasma phospholipids, and 
synthesis of pro-thrombotic cyclo-oxygenase-derived products (thromboxane A2 and B2, PGE2) ${ }^{(647)}$; immune system, cell signalling and gene expression $(648,649)$

Glutathione (reduced, GSH):

Health and diseases ${ }^{(650)}$; source of cysteine ${ }^{(651)}$; oral cancer, anti-carcinogen, antioxidant effect, binding with cellular mutagens and GSH transferase activity ${ }^{(10)}$; detoxification of toxic electrolytic metabolites, xenobiotics and reactive oxygen intermediates ${ }^{(652)}$; cellular immune function $^{(208)}$

Sulfur amino acids:

Methionine:

Precursor of glutathione ${ }^{(200)}$; precursor of $S$-adenosyl methionine ${ }^{(653)}$; neural tube defects ${ }^{(654)}$; colon cancer $^{(410)}$; cognitive impairment in situation of folate deficiency ${ }^{(653)}$; antioxidant activity ${ }^{(655)}$; lipotrope ${ }^{(239)}$

Cystine:

Hair and nail development ${ }^{(656,657)}$; muscle wasting ${ }^{(658)}$; antioxidant and cell signalling through reactive cysteine residues in proteins ${ }^{(659)}$

Total fibre ${ }^{(18,46,58,266,660,661)}$ :

Type 2 diabetes risk ${ }^{(662)}$; risk of weight and fat gains; large bowel cancer ${ }^{(66,75,106,266)}$; satiating effect; cholesterol, bile acids, hormonal activity; immune system, toxicant transit; production of SCFA in the colon ${ }^{(663)}$; SCFA, growth of tumour cells, glutathione-S-transferase and genotoxic activity of 4-hydroxynonenal ${ }^{(664)}$; dilution of gut substances; energy content and glycaemic index of foods; insulin response; free radicals ${ }^{(93)}$

Insoluble fibre ${ }^{(63)}$ : antioxidant-bound phenolics and colon $^{(150)}$; faecal wet and dry weight and faecal bulking effect $^{(660)}$; intestinal transit ${ }^{(660)}$

Soluble fibre ${ }^{(63)}$ : cholesterol; glucose and insulin responses $^{(412)}$; bowel health ${ }^{(412)}$

Lignins:

Antioxidant $(112,149,224)$; dietary carcinogens adsorption $^{(69,266)}$; bile acid reabsorption ${ }^{(268)}$; bile-salt sequestrating agent ${ }^{(107,108)}$; fat absorption ${ }^{(665)}$; bile salt pool size $^{(666)}$; cholesterol turnover ${ }^{(667)}$; formation of carcinogenic metabolites from bile salts ${ }^{(269)}$; precursor of lignans ${ }^{(221)}$; anti-carcinogenic ${ }^{(265)}$

Oligosaccharides (raffinose, stachyose and fructans) ${ }^{(295)}$ :

Serum cholesterol ${ }^{(46,80)}$; gut modifier, enzyme modulator and binding scavenger ${ }^{(46)}$

Fructans $^{(668,669)}$ :

Lifespan and weight gain reduction ${ }^{(670)}$; prebiotic $^{(18)}$; microbiota $^{(671)}$; growth of harmful bacteria, immune system, absorption of minerals and synthesis of $\mathrm{B}$ vitamins $^{(18)}$; absorption of $\mathrm{Ca}, \mathrm{Mg}$ and $\mathrm{Fe}^{(18,72,73)}$; butyrate with cancer-preventing properties in the colon $^{(672)}$; growth of cancer cells ${ }^{(672-674)}$; glycaemia and insulinaemia ${ }^{(668)}$; plasma TAG and total/LDLcholesterol $^{(675,676)}$; lipid metabolism ${ }^{(677)}$; hepatic gluconeogenesis and glycolysis ${ }^{(69)}$

Raffinose: weight gain ${ }^{(297)}$
Arabinoxylans ${ }^{(664)}$ :

Colon cancer growth and progression ${ }^{(678)}$; glucose response ${ }^{(411)}$; chemoprotection and fermentation products $^{(664)}$; bile acids ${ }^{(664)}$; anti-proliferative properties of butyrate ${ }^{(679)}$

$\beta$-Glucans ${ }^{(56)}$ :

Satiety ${ }^{(54)}$; blood sugar and gastric emptying rate ${ }^{(18)}$; blood cholesterol $^{(18)}$; hypoglycaemic and hypoinsulinaemic $^{(680-682)}$; hypocholesterolaemic ${ }^{(56,683)}$; propionate, hepatocyte lipid synthesis and cholesterolaemia ${ }^{(684)}$; anti-carcinogenic $^{(391)}$; immune system ${ }^{(391)}$; peripheral blood monocytes and breast cancer ${ }^{(685)}$; anti-bacterial, anti-parasitic, anti-fungal and anti-viral ${ }^{(391)}$

Phytic acid:

Risk of colon ${ }^{(100)}$ and breast $^{(101)}$ cancers; anti-cancer agent $^{(95,99,106,686)}$; antioxidant activity ${ }^{(148)}$; chelation with various metals and Fenton reaction ${ }^{(95)}$; oxidative damage to the intestinal epithelium and neighbouring cells (cited in Slavin ${ }^{(63)}$ ); lipid peroxidation (cited in Ferguson \& Harris ${ }^{(69)}$ ); formation of ADP-iron-oxygen complexes that initiate lipid peroxidation ${ }^{(687)}$; cellular and nuclear signalling pathways ${ }^{(95)}$; plasma glucose (cited in Yoon et al. ${ }^{(182)}$ ); insulin and/or plasma cholesterol and $\mathrm{TAG}^{(688-690)}$; lipid levels in liver and serum $^{(691)}$; detoxification capacity of liver and levels of GSH transferase and cytochrome P-450 ${ }^{(692)}$; immune response ${ }^{(99)}$; renal stones ${ }^{(693)}$; calcification of cardiovascular system $^{(694)}$; dental caries and platelet aggregation, treatment of hypercalciura and kidney stones, and $\mathrm{Pb}$ poisoning $^{(218)}$; gene expression ${ }^{(695,696)}$

Resistant $\operatorname{starch}^{(697)}$ :

Physically inaccessible within small intestine ${ }^{(18)}$; prebiotic $^{(415)}$; glycaemic response ${ }^{(52)}$; glucose metabolism and plasma NEFA ${ }^{(54)}$; energy intake; SCFA, butyrate and colon health, and SCFA and serum cholesterol ${ }^{(65,80)}$; lipid oxidation and metabolism $^{(67)}$; gallstones ${ }^{(698)}$

Fe:

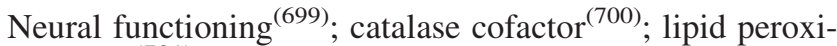
dation ${ }^{(701)}$; cofactor, enzymes and energy metabolism $^{(702)}$; cellular energy metabolism ${ }^{(703)}$; infection and mental function ${ }^{(704)}$; cognitive development and intellectual performance ${ }^{(705,706)}$; collagen synthesis ${ }^{(707)}$; bone health ${ }^{(708)}$; aerobic endurance exercise ${ }^{(709)}$; immunity and infection ${ }^{(710)}$; vitamin metabolism ${ }^{(711)}$; serum and liver TAG, phospholipid, and cholesterol ${ }^{(701)}$; obesity $^{(712)}$

$\mathrm{Mg}^{(204,603)}$ :

Metalloenzymes ${ }^{(569)}$; alkaline phosphatase (bone health) ${ }^{(713)}$; antioxidant ${ }^{(714)}$; lipid peroxidation ${ }^{(715)}$; hypertriacylglycerolaemia ${ }^{(716)}$ and insulin resistance $^{(156,159,715,717,718)}$; diabetes ${ }^{(157,719-722)}$; glucose uptake $^{(158)}$, glucose metabolic clearance rate and insulin response ${ }^{(158,159)}$, and oxidative glucose metabolism ${ }^{(723)}$; platelet aggregability ${ }^{(170)}$; blood pressure regulation ${ }^{(171)}$; coronary atherosclerosis and acute thrombosis ${ }^{(169)}$; vascular function ${ }^{(724)}$; blood pressure ${ }^{(725)}$; cardiovascular 
death rate $^{(726)}$; osteoporosis ${ }^{(727)}$; angiogenesis and inflammation $^{(728)}$; stone formation ${ }^{(729)}$

$\mathrm{Zn}^{(204,700)}$ :

Alkaline phosphatase cofactor; antioxidant and superoxide dismutase (SOD) cofactor ${ }^{(730,731)}$; skeletal growth and maturation, and bone metabolism ${ }^{(732)}$; chemical inactivator $^{(46)}$; formation of active carcinogenic compounds ${ }^{(93)}$; Zn-binding compounds and cancer cell death $^{(733)}$; oesophagus cancer ${ }^{(734)}$; Zn sensing receptor and cell signalling ${ }^{(735)}$; immune functions ${ }^{(736)}$; inflammatory diseases and cell signalling mechanisms ${ }^{(737)}$; type 2 diabetes $^{(738)}$; food intake ${ }^{(739)}$

$\mathrm{Mn}^{(569,700)}$ :

Antioxidant ${ }^{(740)}$; metalloenzyme constituent and enzyme activation $^{(569)}$; bone health ${ }^{(732,741)}$; manganese-SOD, NF$\kappa \mathrm{B}$ activation and carcinogenic process ${ }^{(742)}$; manganeseSOD and tumour growth ${ }^{(743)}$

$\mathrm{Cu}^{(204,700)}$

Antioxidant ${ }^{(744)}$; Cu-containing/binding proteins ${ }^{(569)}$; bone health ${ }^{(732,745)}$; central nervous system dysfunction ${ }^{(700)}$; immune and cardiac dysfunctions ${ }^{(700,746,747)}$; heart health $^{(748,749)}$; anti-cancer effect and DNA binding ${ }^{(750)}$; risk of $\mathrm{CHD}^{(751,752)}$

$\mathrm{Se}^{(204) \text { : }}$

Glutathione peroxidase and thioredoxin reductase cofactor; antioxidant $^{(46,93,753)}$; constituent of selenoproteins ${ }^{(754)}$; tumour growth ${ }^{(46,110,754,755)}$; prostate and colon cancer (cited in Reeves et al. ${ }^{(633)}$ ); susceptibility to carcinogens $^{(756,757)}$; apoptotic effects ${ }^{(758)}$; anti-carcinogenic ${ }^{(759)}$; cell membranes and oxidation damage ${ }^{(760)}$; anti-infective ${ }^{(761,762)}$; plasma, liver and erythrocyte GSH peroxidase activity ${ }^{(763)}$; insulin resistance and vascular endothelium $^{(764,765)}$; platelet aggregation ${ }^{(753)}$

$\mathrm{P}^{(204,603,766)}$ :

Kidney health ${ }^{(766,767)}$; colorectal adenoma ${ }^{(768)}$; tooth development $^{(769)}$

$\mathrm{Ca}^{(204,603)}$ :

Colorectal cancer ${ }^{(770,771)}$; signal transduction element ${ }^{(772)}$; cell signalling ${ }^{(773)}$; mitotic events and cell cycle ${ }^{(774)}$; hypertension ${ }^{(603,775,776)}$; stroke risk $^{(777)}$; diabetes risk $^{(718)}$; tooth development ${ }^{(769)}$; energy balance and obesity ${ }^{(778,779)}$

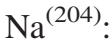

Fluid balance ${ }^{(780)}$; blood pressure ${ }^{(781)} ; \mathrm{CVD}^{(782)}$; osteoporosis and bone health ${ }^{(783)}$

$\mathrm{K}^{(204,784,785) \text { : }}$

Diabetes risk $^{(718)}$; insulin secretion ${ }^{(157,786)}$; blood pressure ${ }^{(787)}$; CVD $^{(788-790)}$; cardiac arrhythmias ${ }^{(791)}$; kidney health ${ }^{(792)}$ and stones $^{(793)}$; bone health ${ }^{(794)}$; hypercalciura ${ }^{(795)}$

Thiamin (vitamin $\left.\mathrm{B}_{1}\right)^{(204,796,797) \text { : }}$

Antioxidant ${ }^{(798)}$; glucose metabolism and Krebs cycle ${ }^{(799)}$; mental and neuronal health ${ }^{(800)}$
Riboflavin (vitamin $\left.\mathrm{B}_{2}\right)^{(204,796)}$ :

Haematopoiesis ${ }^{(801,802)}$; gastrointestinal development ${ }^{(803)}$; mental health ${ }^{(804)}$; vision ${ }^{(805)}$; cardiovascular protection $^{(806,807)}$; cancer $^{(808,809)}$

Niacin (vitamin $\left.\mathrm{B}_{3}\right)^{(204,796)}$ :

Hypolipidaemic and cardiovascular protection ${ }^{(810,811)}$; cancers ${ }^{(812)} ; \operatorname{AIDS}^{(813)}$; $\operatorname{arthritis}^{(814)}$; catecholamine stimulation of lipolysis ${ }^{(815,816)}$ (cited in Marcus et al. ${ }^{(817)}$ and Figge et al. ${ }^{(810)}$ )

Pantothenic acid (vitamin $\left.\mathrm{B}_{5}\right)^{(204,796)}$

Pyridoxine (vitamin $\left.\mathrm{B}_{6}\right)^{(204,796)}$ :

Colorectal cancer ${ }^{(818)}$; asthma and $\mathrm{CVD}^{(819)}$; impaired homocysteine metabolism and occlusive arterial disease $^{(820)}$

Biotin (vitamin $\left.\mathrm{B}_{8}\right)^{(204,796,821-823)}$ :

Regulation of gene expression ${ }^{(824)}$; cell proliferation ${ }^{(825)}$; dermatological abnormalities; immune response ${ }^{(826,827)}$

Folates (vitamin $\left.\mathrm{B}_{9}\right)^{(204,796,828)}$ :

Plasma homocysteinaemia ${ }^{(829,830)}$; neural tube defects $^{(273,831)}$; biochemistry of nucleic $\operatorname{acid}^{(828)}$; colon cancer risk ${ }^{(410,832)}$; anti-carcinogenic ${ }^{(833,834)}$; megaloblastic anaemia ${ }^{(835)}$; depression ${ }^{(274-276)}$; fertility ${ }^{(836)}$; lipotrope ${ }^{(239)}$; methylation and related epigenetic effects on gene expression ${ }^{(837)}$

Tocopherols and tocotrienols (vitamin E) ${ }^{(204)}$ :

Cardiovascular risk ${ }^{(838,839)}$; antioxidant $^{(840-842)}$; Se and reduced state (cited in Slavin ${ }^{(63)}$ ); formation of nitrosamines (cited in Slavin ${ }^{(63)}$ ); formation of carcinogens (cited in Slavin et al. ${ }^{(663)}$ ); $\operatorname{apoptosis}^{(843)}$

Tocopherols:

Non-antioxidant effects ${ }^{(844)}$; chemical inactivator (cited in Kohlmeier et al. ${ }^{(93)}$; protein kinase $\mathrm{C}$ regulation ${ }^{(844,845)}$; monocyte superoxide anion and IL-1 ${ }^{(846)}$; gene expression and cell signalling ${ }^{(844,847,848)}$; peroxynitritederived nitrating species $^{(849,850)}$; cell proliferation ${ }^{(851)}$; pancreatic carcinogenesis ${ }^{(852)}$; type 2 diabetes-induced oxidative stress $^{(853)}$

Tocotrienols $^{(347)}$ :

Neurodegeneration and immune responses ${ }^{(347)}$; cancer $^{(94,347,851)}$; cholesterol ${ }^{(347)}$; risk of heart disease; obesity and osteoporosis/bone calcification ${ }^{(854,855)}$

Phylloquinone (vitamin K) ${ }^{(204,700,856)}$ :

Coenzyme and formation of $\gamma$-carboxyglutamate residues $^{(857)}$; osteoporosis ${ }^{(858)}$; atherosclerosis ${ }^{(859)}$

$\beta$-Carotene:

Cancer ${ }^{(860)}$; colon cancer $^{(106,861)}$; lung cancer $^{(862-864)}$; tumour growth suppressor ${ }^{(824,865)}$; apoptosis $^{(866)}$; immune function ${ }^{(867)}$; antioxidant ${ }^{(868)}$; coronary artery disease risk $^{(869)}$

Lutein (xanthophyll family) ${ }^{(870,871)}$ : 
Ocular function ${ }^{(872)}$; age-related macular degeneration ${ }^{(873)}$; cataract $^{(874)}$; macular pigment density ${ }^{(875)}$; antioxidant ${ }^{(871,876,877)}$; CVD, stroke and lung cancer ${ }^{(862,863)}$. skin protection ${ }^{(878)}$; colon cancer $^{(879)}$; atherosclerosis ${ }^{(880)}$

\section{Zeaxanthin (xanthophyll family):}

Age-related macular degeneration ${ }^{(873)}$; cataract $^{(881)}$; macular pigment density ${ }^{(875)}$; antioxidant ${ }^{(871,876,877)}$; CVD and stroke (cited in Anonymous ${ }^{(876)}$ ); skin protection ${ }^{(878)}$; lung cancer ${ }^{(862)}$

\section{$\beta$-Cryptoxanthin:}

Anabolic effects on bone components and bone loss/resorption $^{(882,883)}$; anti-proliferative/chemopreventive agent and lung cancer ${ }^{(863,884-886)}$; carcinogenesis $^{(887)}$; control of differentiation and apoptosis ${ }^{(888)}$; antioxidant (cited in Castelao \& Olmedilla ${ }^{(889)}$ )

Phenolic acids:

Antioxidant $^{(890)}$; insulin secretion ${ }^{(891)}$; plasma glucose, insulin, cholesterol and TAG (cited in Slavin et al. ${ }^{(46)}$ ); cancer and action as blocking compounds ${ }^{(892)}$; carcinogens binding to targets and release of phenolicbound antioxidant ${ }^{(150,893)}$; tumour growth suppressor (cited in Slavin et al. ${ }^{(46)}$ and Thompson ${ }^{(173)}$ ); enzyme modulators (cited in Slavin et al. ${ }^{(46)}$ ); dyslipidaemia, hepatosteatosis and oxidative stress $^{(894)}$; cell signalling $(186,189)$

Ferulic acid ${ }^{(104,261,262)}$

Antioxidant $^{(895)}$; HDL-cholesterol ${ }^{(896)}$; hyperlipidaemia $^{(897)}$; anti-carcinogenic ${ }^{(69)}$, for example, tongue cancer $^{(892)}$; hypotensive and vascular relaxation ${ }^{(898)}$; hypoglycaemia ${ }^{(899)}$; neurodegenerative disorders (cited in Barone et al. ${ }^{(104)}$ )

Flavonoids:

Antioxidant ${ }^{(69,890)}$; enzyme modulator, antioxidant and tumour growth suppressor (cited in Kohlmeier et al. ${ }^{(93)}$ ); anti-carcinogenic (cited in Ferguson \& Harris ${ }^{(69)}$ and Thompson $\left.{ }^{(173)}\right)$; CVD $^{(900)}$; signalling molecules ${ }^{(188,189,191)}$; cell signalling, gene regulation, angiogenesis and other biological processes ${ }^{(214)}$; inflammation ${ }^{(189)}$; platelet aggregation $^{(901)}$; anti-microbial ${ }^{(902)}$; production of urate ${ }^{(214)}$; bone resorption ${ }^{(903)}$; dyslipidaemia, hepatosteatosis and oxidative stress ${ }^{(894)}$

Anthocyanins:

Antioxidants ${ }^{(904-906)}$; anti-inflammatory ${ }^{(907,908)}$; anti-carcinogenic $^{(909,910)}$; hypoglycaemic ${ }^{(911)}$

Isoflavonoids:

Hormone-like diphenolic phyto-oestrogens ${ }^{(293)}$; cancer and atherosclerosis $^{(293)}$; osteoporosis ${ }^{(293)}$; trabecular connectivity and thickness ${ }^{(912)}$

\section{Lignans:}

Hormone-like diphenolic phyto-oestrogens ${ }^{(293)}$; antioxidant $^{(18,45,69,96)}$; hormonally mediated diseases ${ }^{(293)}$; cell proliferation ${ }^{(97)}$; tumour growth suppressor ${ }^{(913)}$; precursors of enterolactone and enterodiol ${ }^{(96,914,915)}$; cancers $^{(96)}$; osteoporosis ${ }^{(293)}$; rheumatoid arthritis, gastric and duodenal ulcers, skin health, diuretic, antagonistic action of platelet-activating factor receptor and action on superoxide production (cited in Thompson ${ }^{(173)}$ )

Alkylresorcinols ${ }^{(396)}$ :

Antioxidant ${ }^{(916,917)}$; anti-carcinogenic, anti-microbial, antiparasitic and cytotoxic, structure and metabolism of nucleic acids, phospholipid bilayer properties ${ }^{(400)}$; antimutagenic $^{(918)}$; 3-phosphoglycerate dehydrogenase (key enzyme of TAG synthesis in adipocytes) $)^{(398)}$; liver cholesterol $^{(399)}$

Betaine:

Fatty deposits in the liver and hyperhomocysteinaemia ${ }^{(919)}$; osmoprotectant, performance (for example, athletic) ${ }^{(225)}$; organic osmolyte ${ }^{(920)}$; $\mathrm{CVD}^{(921)}$; homocysteine and inflammatory markers related to atherosclerosis (C-reactive protein and $\mathrm{TNF}-\alpha)^{(922,923)}$; sulfur amino acid homeostasis ${ }^{(924)}$; colorectal adenoma ${ }^{(121)}$; antioxidant and non-alcoholic fatty liver diseases ${ }^{(925)}$

Choline ${ }^{(226,796)}$

Brain development and normal memory function ${ }^{(926-928)}$; plasma homocysteine level ${ }^{(929)}$; antioxidant ${ }^{(930)}$; carnitine conservation $^{(931)}$; body fat and fatty acid oxidation ${ }^{(932,933)}$; precursor for the cell membrane phospholipids phosphatidylcholine $^{(934)}$, sphingomyelin ${ }^{(226,935)}$, brain acetylcholine ${ }^{(936)}$ and for platelet-activating-factor formation ${ }^{(937)}$; synthesis and release of acetylcholine ${ }^{(936,938)}$; lipid metabolism, hepatic secretion of VLDL, nerve function and integrity of cell membranes ${ }^{(226)}$; neural tube development ${ }^{(939)}$; lipotrope and methyl donor ${ }^{(240)}$; DNA hypomethylation and tumour development in the liver ${ }^{(226,239,258,940)}$; epigenetic regulator of gene expression ${ }^{(941)}$

Phytosterols ${ }^{(18,942,943)}$ :

Total and LDL serum cholesterol ${ }^{(942,944-947)}$; micelle formation, dietary and biliary cholesterol absorption and LDL-cholesterol ${ }^{(948)}$; vascular smooth muscle cell hyperproliferation ${ }^{(949)}$; immunosuppression associated with excessive physical stress ${ }^{(950)}$; anti-inflammatory, anti-pyretic, immunomodulator and anti-diabetic (cited in Brufau et al. $\left.{ }^{(942)}\right)$; anti-diabetic and hypoglycaemic ${ }^{(951)}$

$\beta$-Sitosterol:

Growth of colon cancer line ${ }^{(952,953)}$; prostate cancer ${ }^{(954)}$; carcinogen-induced neoplasia (cited in Wattenberg ${ }^{(110)}$ ); apoptosis $^{(955)}$ through caspase activation ${ }^{(956)}$

Inositols:

Chiro-inositol:

Insulin, signal transduction and mimetic of insulin action $^{(957)}$; type 2 diabetes ${ }^{(245,958-961)}$; ovulatory functions and serum androgen concentrations, blood pressure and plasma $\mathrm{TAG}^{(62)}$; folate-resistant neural tube defects ${ }^{(962)}$; pinitol and glucose metabolism ${ }^{(622)}$

Myo-inositol:

Metabolism $^{(963)}$; TAG and total lipid liver, hepatic activities of glucose-6-phosphate dehydrogenase, malic enzyme, fatty acid synthetase and citrate cleavage enzyme ${ }^{(242,964)}$; 
conversion into chiro-inositol and precursor for several phospholipids (cited in Larner ${ }^{(957)}$, Novak et al. ${ }^{(965)}$ and Pak et al. ${ }^{(256)}$; mental health ${ }^{(966,967)}$; osmotic demyelination syndrome ${ }^{(968)}$; volume regulation during persistent osmotic stress ${ }^{(969)}$; cancer $^{(686)}$; diabetic polyneuropathy and nerve conduction ${ }^{(970)}$; intestinal lipodystrophy ${ }^{963)}$

Policosanol:

Octacosanol in human health ${ }^{(302)} ; \mathrm{CVD}^{(304)}$; lipid, cholesterol

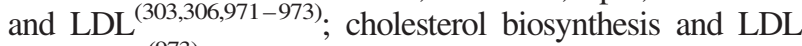
catabolism $^{(973)}$; hydroxymethylglutaryl-coenzyme A (HMG$\mathrm{CoA}$ ) reductase ${ }^{(974)}$; LDL peroxidation ${ }^{(307)}$; membrane lipid peroxidation $^{(975)}$; lipid metabolism ${ }^{(301)}$; platelet aggregation and thromboxane generation, endothelial damage and foam cell formation ${ }^{(976,977)}$; cytoprotection in gastric ulcer ${ }^{(978)}$; athletic performances ${ }^{(979)}$; cardiac events, and cholesterol and anti-aggregatory effects (cited in Taylor et al. ${ }^{(302)}$ ); smooth muscle cell proliferation ${ }^{(980)}$; anti-fatigue drug ${ }^{(302)}$

\section{Melatonin ${ }^{(981)}$ :}

Mood, happiness, sleep and brain neuromodulation in Alzheimer's disease ${ }^{(309,310)}$; antioxidant ${ }^{(982,983)}$; corticoid receptor ${ }^{(984)}$; scavenger of hydroxyl radicals ${ }^{(985)}$; brain GSH peroxidase activity ${ }^{(986)}$; gene expression for antioxidant enzyme ${ }^{(987)}$; sleep-wake regulation ${ }^{(309,988)}$; DNA damage ${ }^{(989)}$; lifespan ${ }^{(99)}$; oncostatic role and antiproliferative effect ${ }^{(311,312)}$; cancers $^{(991)}$

para-Aminobenzoic acid (PABA):

Acetylation in blood and other tissues ${ }^{(315,321,992,993)}$; peroxisomal $\beta$-oxidation and PABA acetylation ${ }^{(316)} ; \mathrm{N}$-acetyltransferase regulation $^{(994)}$; acetylation $^{(319,320)}$; rickettsial infections and collagen diseases ${ }^{(995)}$; serum cholesterol ${ }^{(995)}$; folate formation $^{(31)}$; treatment of vitiligo, leukaemia, rheumatic fever and in rickettsial diseases(cited in Barbieri et al. ${ }^{(316)}$ ); production of thromboxane ${ }^{(321)}$; anti-aggregatory ${ }^{(996)}$; UV protection of the skin (cited in Barbieri et al. ${ }^{(996)}$ and Wang et al. ${ }^{(315)}$ ); liver folic acid metabolism (cited in Russell et al. ${ }^{\left({ }^{(97)}\right)}$

$\gamma$-Oryzanol ${ }^{(361)}$

Cholesterol and rice bran oil ${ }^{(998)}$; cholesterol absorption and aortic fatty streaks ${ }^{(348,358)}$; lipid metabolism ${ }^{(999)}$; autonomic nervous unbalance and menopausal troubles (climacteric disturbance) $^{(999,1000)}$; anti-ulcerogenic ${ }^{(1001)}$; antioxidant ${ }^{(357,360,1002)}$; gene expression and oxidative stress $^{(1003)}$; glycaemia control ${ }^{(1004,1005)}$; platelet aggregation $^{(1006)}$; anxiety and stress ulcer ${ }^{(1001,1007-1009)}$

Avenanthramides:

Anti-inflammatory and anti-atherogenic ${ }^{(368)}$; smooth muscle cell proliferation and NO production ${ }^{(1010,1011)}$; antioxidant $^{(140,367,369)}$

Saponins $^{(370,1012,1013)}$ :

Hypercholesterolaemia ${ }^{(173,374,377,1014)}$; lipase activity and fat absorption $^{(1015)}$; transcriptional activity of $\mathrm{Cu}, \mathrm{Zn}$-SOD gene ${ }^{(1016)}$; scavenger and superoxides ${ }^{(1017)}$; hypoglycae$\mathrm{mia}^{(1018,1019)}$; gastric emptying rate and glucose transport across the brush border of the small intestine ${ }^{(1018,1020)}$; antifungal $^{(374)}$; anti-viral ${ }^{(1021)}$; diabetes ${ }^{(1022)}$; anti-inflammatory ${ }^{(1019)}$; anti-carcinogenic ${ }^{374)}$; tumour growth and cytostatic effect $^{(1013,1023-1027)}$; bile acid binding (cited in Mimaki et al. ${ }^{(1013)}$; cell-mediated immune system and antibody production ${ }^{(375)}$; nervous system functioning ${ }^{(1012,1028)}$; blood coagulation $^{(1029)}$ 